\title{
Correlaciones estadísticas, interacciones y localización en sistemas cuánticos
}

Tesis para obtener el grado de Doctorado en Ciencias

(Química)

que presenta:

Humberto Laguna Galindo

Asesor: Dr. Robin P. Sagar

Universidad Autónoma Metropolitana

Unidad Iztapalapa

División de Ciencias Básicas e Ingeniería

January 22, 2014 


\section{Agradecimientos}

Tuve mucha suerte de encontrar a Robin Sagar como asesor, mentor y amigo, porque ha sido una guía para descubrir la actividad científica y espero haber aprendido de él la humildad y el gusto sin reservas por la ciencia. Todas las ideas de esta tesis se cocinaron sazonadas con la discusión amena con él, en la que siempre encontré un balance adecuado entre la formalidad y la libertad que permite desarrollar la creatividad. Agradezco mucho sus enseñanzas, estoy seguro que me acompañarán por el camino.

Quiero también agradecer a mis compañeros de generación, Mariano, Ángel, Ariana, Fernando, Francisco, Guillermo y muy especialmente a Alejandro, por todas las pláticas. A Tania, tnkis, mi manita, por las pláticas que nunca pudimos resolver sobre la ciencia y la sociedad. También a mis amigos del área de Química Cuántica y del Departamento de Química, a quienes mencionar sería extenso, pero quiero recordar a Edmundo, y mencionar especialmente a Delia, Corina, Juan y Héctor. A mis profesores

de la licenciatura y especialmente a los profesores del Área de Química Cuántica.

Sería extenso mencionar a todas y todos quienes tuvieron un papel para la conclusión de este trabajo, pero quiero expresar mi agradecimiento por la ayuda, a veces inconsciente.

Finalmente, pero no menos importante, ha llegado la hora de agradecer por los recursos que hicieron posible realizar este trabajo.

Gracias al Conacyt por la Beca Doctoral número 207213 y por la Beca Mixta que me permitió realizar una parte de este trabajo.

Por los recursos para asistir a cuatro Reuniones Mexicanas de Fisicoquímica Teórica, la VIII, la IX, la X y la XII quiero agradecer a los organizadores de la Reunión, al Dr. Robin Sagar y al Área de Química Cuántica. 
Quiero agradecer por los recursos para asistir al 52 Sanibel Symposium al Posgrado Divisional de la DCBI-UAMI y al Área de Química Cuántica.

Por los recursos para asistir al 53 Sanibel Symposium agradezco al ICyT-DF y a la UAM.

Por los recursos para asistir al APS March Meeting 2013 quiero agradecer al Área de Química Cuántica.

También quiero agradecer al convenio 58728 del Conacyt y a la Dra. María Villa por los recursos computacionales con que fueron realizadas partes de este trabajo, a ella le agradezco mucho el apoyo a lo largo de todo mi doctorado. 


\section{UNA DEDICATORIA}

Pienso que en un país donde un tercio de la población vive con el hambre acechándole cada día la democracia no existe. El año pasado en México murieron 10,000 niños a causa de enfermedades relacionadas con la desnutrición, otros tantos, pero fuera de estas estadísticas, morirían de enfermedades curables que extrañamente no se cuentan en el mismo rubro. La grosera convivencia entre la opulencia y la desmesura de unos con la pobreza de otros, "los que nunca van a superarse", orilla a volver la vista hacia los referentes que indican que otro mundo es posible, como dijeron hace más de 20 años los zapatistas.

Entre los ejemplos resalta uno, al que quiero dedicar mi tesis: El pueblo de Cuba. Ese pueblo latinoamericano ha desafiado a la historia y ha mantenido para sí su independencia y autodeterminación. La democracia tiene un lugar más propicio en donde nadie muere por hambre, aún con un bloqueo criminal que pocas naciones en el mundo soportarían.

Soy consciente de los riesgos de confundir los pueblos con los líderes, pero hay ocasiones en que no se trata de una confusión sino de una identidad prácticamente completa. Por ello quiero dedicar el producto de estos años de trabajo al Comandante Fidel Castro Ruz, que nunca ha cejado en su empeño de conquistar y construir la libertad para su pueblo y que toda su vida ha prestado su voz a los condenados de la tierra. Que él siga siendo ejemplo para la juventud.

También quiero dedicar mi trabajo a la huelga del Consejo General de Huelga de la UNAM ocurrida en 1999, porque gracias a esa lucha estudiantil este país aún tiene universidades gratuitas y también porque trazó nuevos caminos en el horizonte para varias generaciones de jóvenes. A mis compañeras y compañeros de esos años junto a quienes aprendí a defender la educación y el sentido real de la democracia y el diálogo.

A mis compañeras y compañeros de organización, con quienes aprendo a caminar

y a construir. A Alo, Ángelita, Charles, Gera, Jime, Ju, Karlita, Mati, Moi, Néstor, Olmo, Pancho, Pix, Santi, Sela, Sofi, Taninha, Tauto y Paloma. 
También quiero incluir a mi familia. A Cristina, con quien decidí caminar el sendero de la vida, a ella por el cariño, por la comprensión y por el ejemplo, y no puedo dejar de agradecerle la corrección de estilo que mejoró mucho esta tesis. A mi hermana y compañera Selene que siempre va un paso adelante.

A mis padres, Micaela y Vicente, porque sobre sus pasos es que estoy aprendiendo a caminar y a ellos debo muchísimo. Gracias. También a mis tíos, Marcial y Tere, que muchas veces hicieron las veces de padres sin tener obligación, muchas gracias. 


\section{Índice}

$\begin{array}{ll}\text { Índice de Figuras } & 10\end{array}$

$\begin{array}{ll}\text { Índice de Tablas } & 17\end{array}$

1 Introducción metodológica $\quad 19$

1.1 Interacciones físicas, indistinguibilidad y no conmutatividad . . . . . . 19

1.2 Correlación y localización . . . . . . . . . . . . . . . 21

1.3 Enfoque del estudio . . . . . . . . . . . . . . . . . . . . . . 22

1.3.1 Correlación estadística . . . . . . . . . . . . . 23

1.3.2 Modelos . . . . . . . . . . . . . . . . . . 25

1.4 Efectos del potencial . . . . . . . . . . . . . . . 26

1.4.1 Indistinguibilidad y potencial . . . . . . . . . . . 27

1.4.2 No conmutatividad y potencial . . . . . . . . . . . . . 29

1.5 Efectos de la simetría de la función de onda . . . . . . . . . . . . . . 30

1.5.1 Indistinguibilidad y no conmutatividad . . . . . . . . . . . 30

1.6 Efectos de la no conmutatividad de los operadores . . . . . . . . . . . . 31

1.7 Separar las correlaciones . . . . . . . . . . . . . . . 32

$2 \quad$ Mecánica cuántica $\quad 33$

2.1 Conceptos generales . . . . . . . . . . . . . . . . . . . . 33

2.2 El Principio de Incertidumbre . . . . . . . . . . . . . . . . . . 38

2.3 El átomo de Moshinsky . . . . . . . . . . . . . . . . . . . . . . . 40 
2.4 Una variación en el modelo . . . . . . . . . . . . . . . . . . . . . . 42

3 Función de Wigner $\quad 44$

3.1 La representación de Dirac . . . . . . . . . . . . . . . . . . . . . . . 44

3.2 El operador de la densidad . . . . . . . . . . . . . . . . . . . 45

3.3 Propiedades de la función de Wigner . . . . . . . . . . . . . . . 47

4 Teoría de la Información $\quad 51$

4.1 La entropía de Shannon . . . . . . . . . . . . . . . . . . 52

4.2 Propiedades de la entropía de Shannon . . . . . . . . . . . . . . . 55

4.3 Teorema de unicidad . . . . . . . . . . . . . . . . 56

4.4 Entropía discreta y entropía diferencial . . . . . . . . . . . . . . 60

4.5 La entropía diferencial de Shannon . . . . . . . . . . . . . . 61

4.6 Problemas observados en las entropías diferenciales . . . . . . . . 63

4.7 Medidas de incertidumbre cuántica . . . . . . . . . . . . . . . . . 64

4.8 Medidas de la correlación estadística en sistemas cuánticos . . . . . . 69

4.9 Relaciones entrópicas de incertidumbre . . . . . . . . . . . . . 70

4.10 Información mutua entre posición y momento, $I_{x, p} \ldots \ldots \ldots 72$

4.11 Entropía de la función de Wigner, $s_{w} \ldots \ldots \ldots \ldots$

4.11 .1 Definición . . . . . . . . . . . . . . . . . . 73

4.11 .2 Interpretación de $s_{w} \ldots \ldots \ldots \ldots \ldots \ldots$

5 Correlaciones estadísticas entre las posiciones y entre los momentos en el Átomo de Moshinsky $\quad 79$

5.1 Estado basal . . . . . . . . . . . . . . . . . . . . . . . . . . . . . 79

5.1 .1 Medidas de localización . . . . . . . . . . . . . . . . 79

5.1 .2 Medidas de correlación . . . . . . . . . . . . . . . . . . 82

5.2 Estado Excitado $\left(n_{R}, n_{r}\right)=(0,1) \ldots \ldots \ldots \ldots$

5.2 .1 Medidas de localización . . . . . . . . . . . . . . . . 83

5.2 .2 Medidas de correlación . . . . . . . . . . . . . . . 85 
5.3 Conclusiones . . . . . . . . . . . . . . . . . . . . . . . . 90

6 Simetría en la función de onda, huecos de simetría, interacciones y correlación estadística $\quad 92$

6.1 Diseño del modelo . . . . . . . . . . . . . . . . . . . . . . . . . . . . . 94

6.2 Simetría de la función de onda y huecos de simetría . . . . . . . . . 96

6.2.1 Localización en la densidad de pares . . . . . . . . . . . 97

6.2.2 Localización en la densidad reducida de una variable . . . . . 105

6.2.3 Correlación estadística . . . . . . . . . . . . . . . . . . . 111

6.3 Simetría en la función de onda, huecos de simetría y números cuánticos 113

6.3.1 Localización en la densidad de pares . . . . . . . . . . . . 120

6.3.2 Localización en la densidad reducida de una variable . . . . . . 121

6.3.3 Correlación estadística . . . . . . . . . . . . . . . . . . . . . . 123

6.4 Otras características en la simetría del modelo . . . . . . . . . . . . . . 124

6.5 Conclusiones . . . . . . . . . . . . . . . . . . . . . . 128

$7 \quad$ El átomo de Moshinsky en el espacio fase: Correlaciones entre posición $\begin{array}{ll}\text { y momento } & 131\end{array}$

7.1 Localización . . . . . . . . . . . . . . . . . . . . . . . . . . . . 131

7.1.1 Distribuciones de dos partículas . . . . . . . . . . . . . 131

7.1.2 Distribuciones reducidas de una partícula . . . . . . . . . . 133

7.2 Correlaciones . . . . . . . . . . . . . . . . . . 135

7.2.1 Correlaciones entre partículas . . . . . . . . . . . . 135

7.2 .2 Correlaciones totales . . . . . . . . . . . . . . . . . 137

7.2 .3 Correlación entre posición y momento . . . . . . . . . . . 138

7.2.4 Relaciones entre las medidas de correlación . . . . . . . . . . . . 141

7.2 .5 Correlación no local $x_{1} \rightarrow p_{2} \ldots \ldots \ldots \ldots \ldots$

7.3 Conclusiones . . . . . . . . . . . . . . . . . . . . 146 
8 Correlación posición-momento en un modelo de una partícula en una $\begin{array}{ll}\text { caja con un potencial efectivo } & 148\end{array}$

8.1 El corral cuántico . . . . . . . . . . . . . . . . . . . . . . . . . . . . . . 148

8.2 Funciones de Wigner . . . . . . . . . . . . . . . . . . . . . . . 149

8.2.1 Entropía de Shannon de la Función de Wigner . . . . . . . . . . 154

8.2 .2 Información Mutua . . . . . . . . . . . . . . . . . 158

8.3 Conclusiones . . . . . . . . . . . . . . . . . . . . . . 160

9 Localización en espacio de posición, momento y espacio fase, y correlación posición-momento en el oscilador armónico confinado 162

9.1 Localización . . . . . . . . . . . . . . . . . . . . . . . . . . . . . . . . . 164

9.1.1 Espacio de posición . . . . . . . . . . . . . . . . . . . 164

9.1 .2 Espacio de momento . . . . . . . . . . . . . . . . 166

9.1.3 Relaciones de incertidumbre . . . . . . . . . . . . . . 170

9.1.4 Función de Wigner . . . . . . . . . . . . . . . . . . . 176

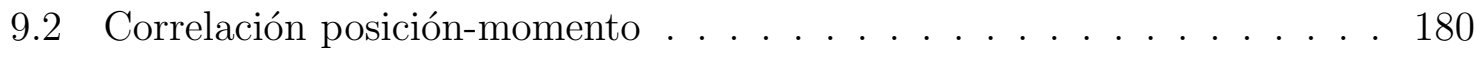

9.3 Resultados analíticos . . . . . . . . . . . . . . . . . . . . . . 181

9.3.1 Localización en los espacios de posición y momento . . . . . . 181

9.3.2 Localización de la función de Wigner y correlación posiciónmomento . . . . . . . . . . . . . . . . . . . . . . 184

9.4 Conclusiones . . . . . . . . . . . . . . . . . . . . . 185

10 Perspectivas para la ampliación de este trabajo 187

10.1 Ampliación a otras funciones de distribución en espacio fase . . . . 187

10.2 Ampliación a variantes del modelo . . . . . . . . . . . . . . . . . 188

10.3 Desarrollo de las herramientas de la teoría de la información . . . . . 189

10.4 Ampliación a otros sistemas . . . . . . . . . . . . . . . . . . . . 189

10.5 El átomo de Moshinsky acoplado con el ambiente . . . . . . . . . . . 190

10.5.1 La ecuación maestra de muchos cuerpos _ . . . . . . . . . 190

10.5.2 Aproximación de Markov y la ecuación maestra de Lindblad . . 191 
10.5.3 El átomo de Moshinsky, un modelo soluble analítico . . . . . . . 192

10.5.4 Localización en la densidad reducida . . . . . . . . . . . . . . 195

10.5.5 Localización en la densidad de pares . . . . . . . . . . . . 196

10.5.6 Correlación estadística . . . . . . . . . . . . . . 197

10.5.7 Perspectivas sobre este modelo . . . . . . . . . . . . . . . . . 198 


\section{Índice de Figuras}

1.1 Representación esquemática de los tres conceptos de la teoría cuántica cuyas relaciones serán estudiadas en esta tesis. . . . . . . . . . . .

4.1 Densidad de probabilidad del estado basal de la partícula en la caja en el espacio de momento, para diferentes valores de tamaño de caja. . . .

4.2 Densidad de momentos, $\pi(p)$, para diferentes estados de la partícula en la caja. Sólo hay variación en la escala para $n=1$. . . . . . . . .

4.3 Desviación estándar (izquierda) y entropía de Shannon (derecha) en el espacio de momento, como función del estado cuántico, de una partícula

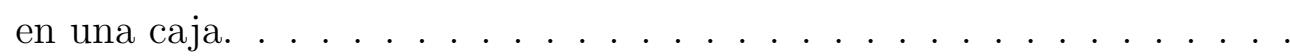

5.1 Entropías de pares, $s_{\Gamma}$ (azul, línea discontinua) y $s_{\Pi}$ (rojo, línea continua) para los estados (a) basal, $(0,0)$, y (b) excitado, $(0,1)$, como funciones del potencial entre partículas, $\kappa$, para potenciales de confinamiento $\omega=0.7,1,1.2 \ldots \ldots \ldots \ldots$

5.2 (CC) Coeficiente de correlación, $\sigma_{x}$ (azul, línea discontinua) y $\sigma_{p}$ (rojo, línea continua) para el estado basal del átomo de Moshinsky $(0,0)$, como

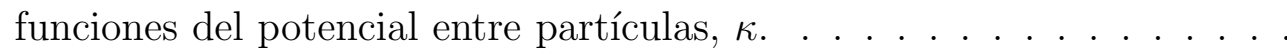

5.3 (MI) Información mutua, $I_{x}$ y $I_{p}$ para el estado basal del átomo de Moshinsky $(0,0)$, como funciones del potencial entre partículas, $\kappa$. Note que $I_{x}=I_{p}$ para este estado. . . . . . . . . . . . 
5.4 (CC) Coeficiente de correlación, $\sigma_{x}$ (azul, línea discontinua) y $\sigma_{p}$ (rojo, línea continua) para un estado excitado del átomo de Moshinsky $(0,1)$, como funciones del potencial entre partículas, $\kappa$. . . . . . . . . . 87

5.5 (MI) Información mutua, $I_{x}$ (azul, línea discontinua) y $I_{p}$ (rojo, línea continua) para un estado excitado del átomo de Moshinsky $(0,1)$, como funciones del potencial entre partículas, $\kappa . \ldots \ldots . . \ldots$

5.6 Análisis de las regiones en la información mutua, $I_{x}$ (izquierda, azul, línea discontinua) y $I_{p}$ (derecha, rojo, línea continua), para un estado excitado del átomo de Moshinsky $(0,1)$, que contienen los puntos donde la correlación aumenta cuando se varía el potencial entre partículas, $\kappa$.

6.1 Entropías de pares para los estados $[(2,1),(1,2)]$ con potencial atractivo (arriba) y con potencial repulsivo (abajo), con $\lambda=0.5$. . . . . . . .

6.2 Densidades de pares para los estados $[(2,1),(1,2)]$ con potencial atractivo (columna izquierda) y con potencial repulsivo (columna derecha), con $\lambda=0.5$ y $\omega=1$.

6.3 Entropías de la densidad reducida de una partícula para los estados $(2,1)$ [azul], y $(1,2)$ [rojo] con potencial atractivo (arriba) y potencial repulsivo (abajo), con $\lambda=0.5 \ldots \ldots \ldots$. . . . . . . . 106

6.4 Densidades reducidas de una partícula para los estados $[(2,1),(1,2)]$ con un potencial atractivo (columna izquierda) y con un potencial repulsivo (columna derecha), con $\omega=1$ y $\lambda=0.5$. . . . . . . . . . . . . 110

6.5 Información mutua para los estados $(2,1)$ [azul] y $(1,2)$ [rojo] con potencial atractivo (arriba) y potencial repulsivo (abajo), con $\lambda=0.5$. . 112

6.6 Detalle de los cruces que ocurren en los estados $(2,1)$ [azul] y $(3,0)$ [rojo]. $s_{\pi_{21}}$ y $s_{\pi_{30}}$ con potencial repulsivo (izquierda). $s_{\rho_{21}}$ y $s_{\rho_{30}}$ con potencial atractivo (derecha). . . . . . . . . . . . . . . . 12

6.7 Información mutua para los estados $[(2,1)[\operatorname{azul}],(3,0)[$ rojo] $]$ con potencial atractivo (arriba) y potencial repulsivo (abajo) 
7.1 Entropía de Shannon de la función de Wigner de dos partículas, $s_{W}$, con la parte real (izquierda, rojo) y la parte imaginaria (derecha, azul), como funciones del número cuántico, $n_{r}$, para $n_{R}=0$. También se muestra la suma entrópica de dos partículas, $s_{T}$, (izquierda, azul, discontinua). . .

7.2 Gráficas 3D de la función de Wigner reducida de una partícula para el estado $\left(n_{R}, n_{r}\right)=(0,1)$ con $\omega=1 \mathrm{y} \kappa=-0.4$ (izquierda), $\kappa=2.0$

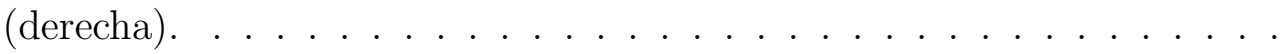

7.3 Suma entrópica de una partícula, $s_{t}$ (roja, línea sólida) y entropía de Shannon de la función de Wigner reducida de una partícula, $s_{w}$ (azul, línea discontinua). Izquierda: como función de $n_{r}$, para $n_{R}=0$ y $\kappa=$ -0.25 . Derecha: como funciones del potencial entre partículas, $\kappa$, para el estado excitado $(0,1) \ldots \ldots \ldots \ldots \ldots$

7.4 Correlación entre partículas, $\operatorname{Re}\left[2 s_{w}-s_{W}\right]$ (rojo, línea sólida), $\left|2 s_{w}\right|-$ $\left|s_{W}\right|$ (verde, línea discontinua), y $\left|2 s_{w}-s_{W}\right|$ (azul, línea punteada). Izquierda: como funciones de $n_{r}$, para $n_{R}=0$ y $\kappa=-0.25$. Derecha: como funciones del potencial entre partículas, $\kappa$, para el estado excitado $(0,1) . I_{x}+I_{p}$ (gris, línea de rayas y puntos) también se muestra. . . . .

7.5 Correlación total, $\operatorname{Re}\left[2 s_{t}-s_{W}\right]$ (rojo, línea sólida), $\left|2 s_{t}\right|-\left|s_{W}\right|$ (verde, línea discontinua), y $\left|2 s_{t}-s_{W}\right|$ (azul, línea punteada). Izquierda: como funciones de $n_{r}$, para $n_{R}=0$ y $\kappa=-0.25$. Derecha: como funciones del potencial entre partículas, $\kappa$, para el estado excitado $(0,1) . I_{x}+I_{p}$ (gris, línea de rayas y puntos) también se muestra. . . . . . . . . .

7.6 Correlación posición-momento en el nivel de dos partículas, $\operatorname{Re}\left[s_{T}-s_{W}\right]$ (rojo, línea sólida), $\left|s_{T}\right|-\left|s_{W}\right|$ (verde, línea discontinua), y $\left|s_{T}-s_{W}\right|$ (azul, línea punteada). Izquierda: como funciones de $n_{r}$, para $n_{R}=0 \mathrm{y}$ $\kappa=-0.25$. Derecha: como funciones del potencial entre partículas, $\kappa$, para el estado excitado $(0,1)$. . . . . . . . . . . . . . 139 
7.7 Correlación posición-momento en el nivel de una partícula, $s_{t}-s_{w}$. Izquierda (en rojo), como función de $n_{r}$, para $n_{R}=0$ y $\kappa=-0.25$. Derecha (en azul), como función del potencial entre partículas, $\kappa$, para el estado excitado $(0,1)$. . . . . . . . . . . . . . . 140

$7.8 I_{b}-\left(I_{x}+I_{p}\right)-I_{x p}^{1}$, con la parte real (rojo) y la diferencia de las normas (azul). Izquierda: como una función de $n_{r}$ para $n_{R}=0$ y $\kappa=-0.25$. Derecha: como función del potencial entre partículas, $\kappa$, para el estado excitado $(0,1)$. . . . . . . . . . . . . . . . 143

$7.9 I_{b}-I_{p}-I_{x p}^{1}$ (dos gráficas de arriba) y $I_{b}-I_{x}-I_{x p}^{1}$ (dos gráficas de abajo), con la parte real (rojo) y la diferencia de las normas (azul). Izquierda: como función de $n_{r}$ para $n_{R}=0$ y $\kappa=-0.25$. Derecha: como función del potencial entre partículas, $\kappa$, para el estado excitado $(0,1)$. . . . .

8.1 Perspectiva superior de las gráficas de la función de Wigner del modelo PIAB (primer renglón) y del modelo radial con $k=0$ (segundo renglón), $k=1$ (tercer renglón) y $k=3$ (cuarto renglón). Las columnas corresponden a $n(m)=1,2,5$. Las gráficas corresponden a valores de $r$ en el intervalo $(0,1)$ y valores de $p$ entre $(-15,15) \ldots \ldots . . . . . . .$.

8.2 Perspectiva lateral de las gráficas de la función de Wigner del modelo PIAB (primer renglón) y del modelo radial con $k=0$ (segundo renglón), $k=1$ (tercer renglón) y $k=3$ (cuarto renglón). Las columnas corresponden a $n(m)=1,2,5$. El cero de la función de Wigner se encuentra a la mitad del eje vertical. Las gráficas corresponden a valores de $r$ en

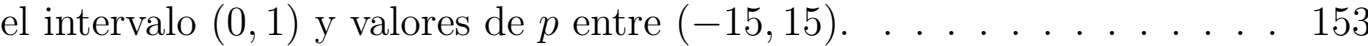

8.3 Gráficas de las tres posibilidades para la entropía de Shannon de la función de Wigner y de $s_{t}$ como funciones de $n(m): k=0$ (verde), PIAB (rojo), $k=1$ (azul). . . . . . . . . . . . . 155 
8.4 Gráficas de las tres posibilidades para la entropía de Shannon de la función de Wigner como funciones de $k: n=1$ (verde), $n=2$ (azul), $n=3$ (rojo), $n=4$ (azul claro), $n=5$ (negro). Hemos amplificado la escala de las tres curvas de abajo, corresponden a $n=2$ con potenciales repulsivos $k \geq 1$. . . . . . . . . . . . . . . . . 157

8.5 Gráficas de las tres posibilidades para la Información mutua como funciones de $n(m): k=0$ (verde), PIAB (rojo), $k=1$ (azul). . . . . . . 158

8.6 Gráficas de las tres posibilidades para la Información mutua como funciones de $k: n=1$ (verde), $n=2$ (azul), $n=3$ (rojo), $n=4$ (azul claro), $n=5$ (negro). La curva de abajo corresponde a $\left|s_{t}-s_{w}\right|$, para $n=2$ con potenciales repulsivos $k \geq 1$, hemos amplificado la escala. . .

9.1 $\Delta x$ como función de $a$ para diferentes valores de $n, n=0$ es rojo y los otros valores son ascendentes conforme $n$ crece. . . . . . . . . . . . . 164

$9.2 s_{x}$ como función de $a$ para diferentes números cuánticos, $n=0$ es azul y los otros valores son ascendentes conforme $n$ crece. . . . . . . . .

$9.3 s_{x}$ como función de $n$ para diferentes valores de $a . a=1 / \sqrt{2}$ (azul), $a=2$ (verde), $a=5$ (rojo) . . . . . . . . . . . . 166

9.4 $\Delta p$ como función de $a$ para diferentes valores de $n, n=0$ es rojo y los otros valores son ascendentes conforme $n$ crece. . . . . . . . . . . . 167

9.5 $s_{p}$ como función de $a$ para diferentes números cuánticos $n=0$ es azul y los otros valores son ascendentes conforme $n$ crece. . . . . . . . . . . 167

$9.6 \pi(p)$. De izquierda a derecha, $n=0, n=2, n=5$; de arriba hacia abajo: PIAB, $a=0.5, a=1, a=3, a=5, \mathrm{HO} . \ldots \ldots$

$9.7 s_{p}$ como función de $n$ para diferentes valores de $a . \quad a=1 / \sqrt{2}$ (azul), $a=2$ (verde), $a=5$ (rojo) . . . . . . . . . . . . . 170

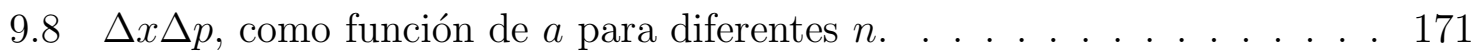

$9.9 s_{t}$ como función de $a \cdot n=0$ es azul y aumenta con el número cuántico. 172 
9.10 Izquierda. $s_{t}^{C H O}$ (rojo) como función de $a$ con $n=0$. Derecha. $s_{t}^{C H O}$ (rojo) como función de $a$ con $n=5$. Además se muestran $s_{t}^{P I A B}$ (azul) y $s_{t}^{H O}$ (verde). . . . . . . . . . . . . . . . . 172

9.11 Detalles de $s_{x}$ (rojo) y $s_{p}$ (azul) como funciones de $a$ de donde se puede inferir el papel que juega el mínimo en el comportamiento. . . . . . . . 173

$9.12 s_{t}$ como función de $n$, con $a=1 / \sqrt{2}$ (azul), $a=2$ (verde), $a=5$ (rojo). 174

$9.13 \rho(x) \pi(p)$. De izquierda a derecha, $n=0, n=2, n=5$; de arriba hacia abajo: PIAB, $a=0.7, a=1, a=3, a=5, \mathrm{HO} . \ldots \ldots 175$

$9.14 R e\left[s_{w}\right]$ (izquierda) y $\operatorname{Im}\left[s_{w}\right]$ (derecha) como funciones de $a . \ldots$. . . . . 176

$9.15 R e\left[s_{w}\right]$ para $n=0$ (izquierda) y $n=5$ (derecha) como funciones de $a . \quad 177$

$9.16 \operatorname{Im}\left[s_{w}\right]$ para $n=0$ (izquierda) y $n=5$ (derecha) como funciones de $a . \quad 177$

$9.17 \operatorname{Re}\left[s_{W}\right]$ (azul), $\operatorname{Im}\left[s_{W}\right]$ (verde) y $\left|s_{W}\right|$ (rojo) . . . . . . . . . . . . 178

9.18 $W(x, p)$. De izquierda a derecha, $n=0, n=2, n=5$; de arriba hacia abajo: PIAB, $a=0.7, a=1, a=3, a=5$, HO. . . . . . . . 179

9.19 Tres posibilidades de $s_{t}-s_{w}$. En cada gráfica $n=0$ (rojo), $n=1$ (verde) y $n=5$ (azul).

$9.20 R e\left[s_{t}-s_{W}\right]$ (azul), $\left|s_{t}\right|-\left|s_{W}\right|$ (verde) y $\left|s_{t}-s_{W}\right|$ (rojo) como funciones

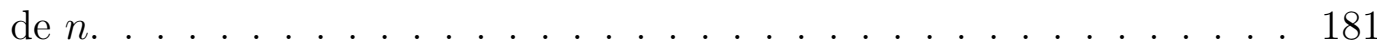

9.21 Izquierda. Gráfica de las entropías en la Tabla (9.2), con $E_{n}=\frac{21}{2}$. Derecha. Gráfica de las entropías del estado basal del CHO, variando $E_{n}$. Ambas curvas se presentan como función de $x_{c} \ldots \ldots \ldots$. . . . .

$10.1 s_{\rho}$ (ordenadas) como función del tiempo (abscisas) para el caso con desfasamiento puro sin relajación (azul) y para el caso con relajación sin desfasamiento puro (rojo). $\lambda=0.01$ (izquierda), $\lambda=0.5$ (centro), $\lambda=0.7$

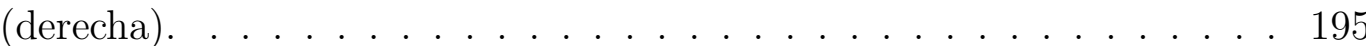


$10.2 s_{\Gamma}$ (ordenadas) como función del tiempo (abscisas) para el caso con desfasamiento puro sin relajación (azul) y para el caso con relajación sin desfasamiento puro (rojo). $\lambda=0.01$ (izquierda), $\lambda=0.5$ (centro), $\lambda=0.7$ (derecha) . . . . . . . . . . . . . . 196

$10.3 I_{x}$ (ordenadas) como función del tiempo (abscisas) para el caso con desfasamiento puro sin relajación (azul) y para el caso con relajación sin desfasamiento puro (rojo). $\lambda=0.01$ (arriba), $\lambda=0.5$ (centro), $\lambda=0.7$

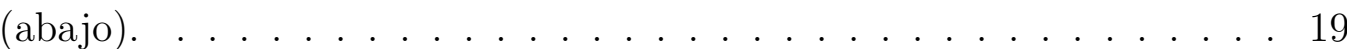

$10.4 I_{x}$, efecto del potencial, $\lambda=0.01$ (azul), $\lambda=0.7$ (rojo). Para el caso con desfasamiento puro sin relajación (izquierda), para el caso con relajación sin desfasamiento puro (derecha). . . . . . . . . . . . . . . . 198 


\section{Índice de Tablas}

5.1 Análisis del coeficiente de correlación y de la información mutua para el estado excitado del átomo de Moshinsky $(0,1) \ldots \ldots$. . . . . . . . 88

6.1 Comparación de los estados $(2,1)$ y $(1,2)$. . . . . . . . . . . . . 98

6.2 Comparación de los estados $(0,1)$ y $(1,0)$. . . . . . . . . . . 103

6.3 Comparación de los estados $(0,3)$ y $(3,0)$. . . . . . . . . . . . 104

6.4 Comparación de los estados $(2,3)$ y $(3,2)$. . . . . . . . . . . . 105

6.5 Comparación entre los estados $(2,1)$ y $(3,0)$. Los valores de $1 / \omega$ para los puntos de cruce se indican entre paréntesis y son aproximados. . . . 115

6.6 Comparación entre los estados $(1,1)$ y $(2,0)$. Los valores de $1 / \omega$ para los puntos de cruce se indican entre paréntesis y son aproximados. . . . 116

6.7 Comparación entre los estados $(1,1)$ y $(0,2)$. Los valores de $1 / \omega$ para los puntos de cruce se indican entre paréntesis y son aproximados. . . . 117

6.8 Comparación entre los estados $(0,3)$ y $(1,2)$. Los valores de $1 / \omega$ para los puntos de cruce se indican entre paréntesis y son aproximados. . . . 118

6.9 Comparación entre los estados $(3,1)$ y $(2,2)$. Los valores de $1 / \omega$ para los puntos de cruce se indican entre paréntesis y son aproximados. . . . 119

6.10 Comparación entre los estados $(1,3)$ y $(2,2)$. Los valores de $1 / \omega$ para los puntos de cruce se indican entre paréntesis y son aproximados. . . . 120

6.11 Comparación entre los estados $(2,0)$ y $(0,2)$. . . . . . . . . . . 124

6.12 Comparación entre los estados $(1,3)$ y $(3,1) \ldots \ldots$. . . . . . . . 125 
6.13 Comparación entre los estados $(1,2)$ y $(3,0)$. Los valores de $1 / \omega$ para los puntos de cruce se indican entre paréntesis y son aproximados. . . . 127

6.14 Comparación entre los estados $(0,3)$ y $(2,1)$. Los valores de $1 / \omega$ para los puntos de cruce se indican entre paréntesis y son aproximados. . . . 128

9.1 Se muestran algunas funciones de onda cuyas transformadas son analíticas y las energías asociadas . . . . . . . . . . . . . . . . . . 183

9.2 Valores de las entropías en los espacios de posición, $s_{\rho}$ y momento $s_{\pi}$ conforme cambia el radio de confinamiento, $x_{c}$ para $E_{n}=\frac{21}{2} \ldots \ldots$. . . 183

9.3 Comparación entre el Oscilador Armónico Confinado y el Oscilador Armónico . . . . . . . . . . . . . . . . . 185 


\section{Introducción metodológica}

\subsection{Interacciones físicas, indistinguibilidad y no con- mutatividad}

Las teorías científicas de la física intentan dar cuenta de las complejas relaciones entre los diversos componentes de la naturaleza. Para ello es necesario capturar sus características en conceptos, que frecuentemente tienen una expresión matemática, y tratar de establecer, también matemáticamente las relaciones entre ellos de manera que ayuden a comprender, explicar, predecir y finalmente controlar la ocurrencia de los fenómenos.

La mecánica cuántica es una teoría física del mundo microscópico que formula las leyes de los fenómenos que ocurren en su ámbito de aplicación. Tres conceptos muy importantes de la teoría cuántica son:

1. Las interacciones debidas a la existencia de un potencial que da lugar a una fuerza:

- La interacción entre partículas ocurre por medio de potenciales, dos ejemplos de ello, ambos muy importantes, son el potencial de Coulomb y el potencial armónico.

- Por medio de un potencial se puede confinar a una o a varias partículas. Dos tipos muy importantes de confinamiento son: 1) con paredes impenetrables que restringen al sistema en una región del espacio y 2) confinamiento 
por medio de un potencial central, como una trampa armónica o una de Coulomb.

2. La imposibilidad de distinguir dos partículas idénticas conduce a la simetrización de la función de onda. La función de onda debe ser simétrica o antisimétrica bajo el intercambio de coordenadas de dos partículas cualesquiera para garantizar que el estado no cambie y por lo tanto que éstas sean indistinguibles. Para una función antisimétrica de partículas con espín (llamadas fermiones) es necesario considerar el principio de exclusión de Pauli.

3. La no-conmutatividad de algunos operadores en sistemas de una o varias partículas. La no-conmutatividad de los operadores asociados a la posición y al momento de una partícula es de especial importancia y conduce al principio de indeterminación de Heisenberg y a las relaciones de incertidumbre.

La enumeración por separado de estos tres conceptos no implica que existan de manera independiente en la naturaleza o que sus relaciones puedan ser despreciadas. No obstante, esta separación implica una manera de analizar metodológicamente un problema, es decir proceder analíticamente (análisis en el sentido de separar el todo en elementos particulares), estudiarlos por separado y luego proceder sintéticamente (síntesis en el sentido de poner junto) estudiando sus relaciones. Estos pasos son elementos comunes de lo que podría llamarse el método científico.

Un esquema que da cuenta de estos tres elementos y de sus relaciones es el siguiente: 
(I) Interacciones físicas

(II) Indistinguibilidad
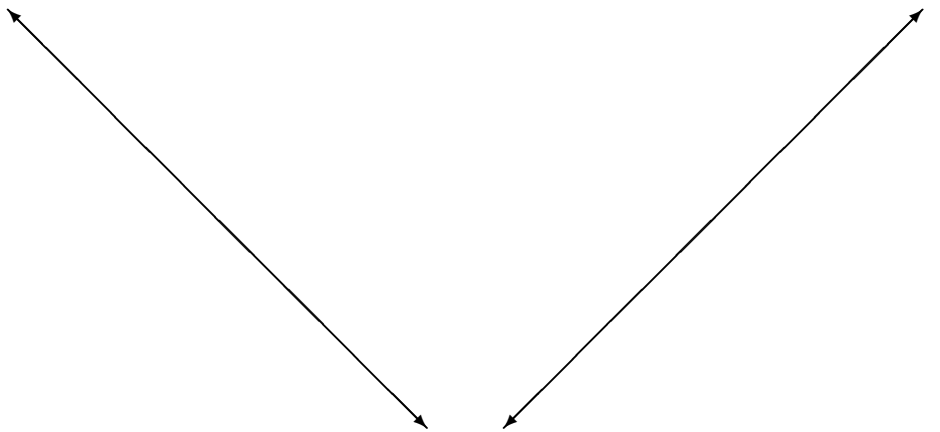

(III) No conmutatividad

Figura 1.1: Representación esquemática de los tres conceptos de la teoría cuántica cuyas relaciones serán estudiadas en esta tesis.

\subsection{Correlación y localización}

Por otro lado, cuando aplicamos la teoría cuántica a los problemas físicos se postula que toda la información del sistema está contenida en la función de onda. Sin embargo, la función de onda no tiene una interpretación directa sino que es su norma cuadrada la que se relaciona con la probabilidad de ocurrencia de un evento (por ejemplo encontrar una partícula en algún punto del espacio o con un momento determinado, etcétera). En el análisis aparecen dos características del sistema (que también son conceptos) con los que se intenta explicar una multiplicidad de fenómenos físicos:

I. La correlación.

- Correlación entre partículas, o más precisamente entre las variables que las describen en alguna representación.

- Correlación entre variables que describen a una misma partícula.

II. La localización, o más precisamente la estructura de la distribución que describe al sistema en alguna representación, por ejemplo la localización en el espacio de 
posición, en el espacio de momento, o en el espacio fase.

Enfocados en el problema de la correlación, se puede estar interesado en estimar: cómo dos partículas están correlacionadas, cuál es la magnitud de la correlación, cuáles son los efectos que las correlaciones entre partículas producen en el sistema en su conjunto, cuáles los que el resto de los componentes del sistema de estudio provocan en las correlaciones entre ellas (por ejemplo, a menudo hablamos un potencial efectivo), etcétera.

Enfocados en el problema de la localización podemos estar interesados en los factores que provocan que la distribución se localice en ciertas regiones del espacio real, o del espacio fase, o del de momento. Por ejemplo, la existencia de regiones en el espacio real en donde está localizada la densidad electrónica se vincula con el enlace químico, o con la estructura de capas atómicas.

\subsection{Enfoque del estudio}

El presente trabajo es un análisis que conjunta los tres conceptos de la teoría cuántica que mencionamos con la correlación y la localización en sistemas cuánticos, es decir

los potenciales, la indistinguibilidad y la no-conmutatividad. Para realizar este análisis debemos seleccionar un enfoque. Utilizaremos un enfoque estadístico para abordar los conceptos de correlación y localización.

La existencia de potenciales, la indistinguibilidad y la no-conmutatividad serán tratados como factores que influyen en la correlación entre algunas variables relevantes de los sistemas que trataremos y en la localización de las distribuciones que los describen.

Las herramientas clásicas de la estadística son la desviación estándar para estudiar la localización de una distribución (que se relaciona directamente con conceptos como incertidumbre, estructura, dispersión) y el coeficiente de correlación para estudiar la correlación estadística entre variables en una distribución. 
Además de esas herramientas estudiaremos otras que provienen de la teoría de la información, en la que la medida de la localización (incertidumbre) es la entropía de Shannon y la medida de la correlación estadística es la información mutua. La correspondencia es:

- Desviación estándar $\rightarrow$ Entropía de Shannon.

- Coeficiente de correlación $\rightarrow$ Información mutua.

Dos preguntas que pueden formularse a partir de lo anterios y que atraviesan varios capítulos del trabajo que ahora se presenta son:

a) ¿Cuáles herramientas son más convenientes para estudiar la incertidumbre cuántica?, cuya respuesta queda abierta puesto que es una discusión vigente [1].

b) ¿Qué medida de la correlación estadística es la más adecuada?

En el marco del enfoque estadístico es necesario discutir el concepto de correlación para clarificar a qué nos referiremos con éste a lo largo de este trabajo.

\subsubsection{Correlación estadística}

Si la descripción de un determinado problema requiere de dos (o más) variables, se dice que, éstas están estadísticamente correlacionadas si su función de distribución conjunta no puede separarse como un producto de funciones de cada una de ellas.

El concepto de correlación estadística requiere entonces de la existencia de una función de distribución conjunta que sea no separable. Si la función es separable a las variables se les llama estadísticamente independientes.

En la mecánica cuántica un efecto de los potenciales es provocar que la ecuación de Schrödinger no pueda separarse y por lo tanto que su solución no sea separable en las coordenadas originales que describen a las partículas. A menudo ésta puede separarse 
con un cambio de coordenadas pero aún cuando este sea el caso hay restricciones de indistinguibilidad que deben cumplirse para el intercambio de las variables originales.

Para enfrentar este problema se utiliza la idea de separabilidad como una primera aproximación su solución. Uno de los tipos de teorías a las que da lugar la idea de separabilidad son las teorías de campo autoconsistente, en las que se asume que la ecuación de Schrödinger de N partículas puede separarse en N ecuaciones de una partícula, cada una de estas con un campo promedio que es producido por el resto. La teoría de Hartree-Fock es la teoría de campo autoconsistente más conocida en el contexto de la química cuántica.

\section{Energía de correlación}

Löwdin definió la energía de correlación como la diferencia entre la energía del sistema de Hartree-Fock y la energía exacta (no relativista) que en la práctica se obtiene por medio de cálculos más refinados. Esta es posiblemente la definición de energía de correlación más utilizada en el contexto de la química cuántica.

La energía de Hartree-Fock es la energía de un sistema de campo autoconsistente que no incluye la interacción instantánea entre las partículas. La diferencia con el sistema exacto debe ser una medida de la no separabilidad de la función de onda que es debida a esa interacción instantánea.

Sin embargo, el sistema de Hartree-Fock sí incluye la indistinguibilidad de las partículas. Una función separable es la solución del sistema de Hartree la cual se escribe como un producto de funciones de una partícula y no existe correlación estadística entre ellas. La diferencia entre la solución de Hartree y la de Hartree-Fock es que la segunda incluye la simetría de la función de onda y por ello es capaz de recuperar comportamientos que la primera no puede.

La solución de Hartree-Fock no es separable como un producto de funciones de una partícula lo cual implica que éstas están estadísticamente correlacionadas aunque se encuentren en un campo promedio. La diferencia entre las soluciones de Hartree y de Hartree-Fock es la correlación que se debe a la indistinguibilidad (primordialmente) 
y la diferencia entre Hartree-Fock y el sistema exacto es la correlación que se debe principalmente a los potenciales.

Esta es una perspectiva energética para abordar el problema de la correlación. La energía de correlación es una cantidad global, es decir, es un número.

\section{Análisis de las densidades}

Hemos vinculado el concepto de correlación estadística con la existencia de una distribución de probabilidad conjunta. La mecánica cuántica puede ser representada en los espacios de posición y momento; en cada uno de ellos la norma cuadrada de la función de onda se interpreta como la densidad de probabilidad de que las partículas tengan determinadas posiciones o momentos.

De manera complementaria a la perspectiva energética para el estudio de la correlación tenemos la perspectiva que se enfoca en cómo la correlación afecta las diferentes representaciones del sistema, es decir, cómo se manifiesta en el espacio de posición y cómo lo hace en el espacio de momento. Así, al analizar las densidades podemos obtener información de la correlación entre las posiciones de un par de partículas o de la correlación entre sus momentos, misma que no puede extraerse directamente de la energía de correlación.

\subsubsection{Modelos}

Utilizaremos tres modelos para los análisis que se desarrollarán en esta tesis, todos en una dimensión y con la característica de que todos tienen una solución analítica en el espacio de posición.

- Una partícula confinada en una caja sujeta a un potencial efectivo que puede ser atractivo o repulsivo. Con este modelo podemos establecer contrastes entre una partícula en una caja y los dos regímenes de potencial.

- El oscilador armónico confinado. Con este modelo podemos estudiar por una parte la influencia de las paredes en un potencial armónico, y por otra la infuencia 
de un potencial armónico en una partícula en una caja. Se trata de un modelo intermedio entre una partícula en una caja y un oscilador armónico, el cual permite estudiar las transiciones de las propiedades al transitar de un modelo a otro.

- El átomo de Moshinsky. Es un sistema de dos partículas interactuando con un potencial armónico confinadas por una trampa armónica. Con este modelo podemos estudiar la influencia del potencial de confinamiento, del potencial entre partículas y los efectos que tiene la simetría en algunas de sus propiedades.

A continuación vamos a plantear el contenido de la investigación utilizando el esquema de la Fig. (1.1) para introducir algunas de las preguntas que orientan nuestra indagación en los modelos de una y dos partículas.

\subsection{Efectos del potencial}

La esquina (I) del esquema de la Fig. (1.1) podemos dividirla en 1) las interacciones de confinamiento presentes en los sistemas de una y dos partículas y 2) las interacciones entre partículas presentes en el átomo de Moshinsky.

Sobre las interacciones de confinamiento en sistemas de una y dos partículas podemos formular las siguientes preguntas:

\section{Una partícula}

- ¿Cuál es la influencia de un potencial en la localización de la densidad de una partícula confinada en una caja en el espacio de posición y en el de momento? Si la influencia es distinta, ¿cómo podemos explicar las diferencias?

- En lo que se refiere a la naturaleza del potencial ¿Existen diferencias entre un potencial repulsivo y uno atractivo para una partícula en una caja? 


\section{Dos partículas}

- En el átomo de Moshinsky, ¿cómo influye el potencial de confinamiento en la localización de las densidades de pares en los espacios de posición y momento?

- ¿Cómo influye el potencial de confinamiento en la localización de las densidades reducidas de una partícula de posición y de momento?

- ¿Cómo influye el potencial de confinamiento en las medidas de correlación en los espacios de posición y momento?

Sobre las interacciones entre partículas en el átomo de Moshinsky nuestras preguntas son:

- ¿Cómo afectan la magnitud y el régimen (atractivo o repulsivo) del potencial entre partículas a la localización de las densidades de pares y de las densidades reducidas de posición y de momento?

- ¿Están las posiciones de las partículas más correlacionadas que sus momentos o al revés?

- ¿Cómo este orden se ve influido por el potencial entre las partículas? ¿Es posible controlar el orden usando el potencial?

\subsubsection{Indistinguibilidad y potencial}

En esta sección plantearemos algunas preguntas relacionadas con la interrelación de la simetría (esquina II del esquema en la Fig. (1.1)) y el potencial (esquina I) que abordaremos en el átomo de Moshinsky. Anteriormente hemos estudiado los efectos de la simetría en sistemas no interactuantes [2, 3] por lo que ahora nos interesa enfocarnos en los efectos de las interacciones en la simetría de la función de onda y viceversa.

Se ha sugerido que la antisimetría puede interpretarse como una interacción con un potencial de contacto de magnitud infinita (delta de Dirac) [4, 5], y aunque esto no es 
preciso sí puede usarse como un ejemplo de un potencial que se utiliza para simular el hueco de Fermi que es un efectos de la antisimetría.

La magnitud del potencial entre partículas puede controlarse experimentalmente en contraste con la simetría de la función de onda la cual no es posible utilizar como parámetro. Al respecto podemos decir que variando la magnitud del potencial se provoca que una distribución de partículas distinguibles tenga características de una distribución de partículas indistinguibles [5]. Estos ejemplos se suman a otros que intentan utilizar la simetría de la función de onda como un recurso $[6,7,8,9]$.

En este sentido, las preguntas que planteamos son:

- ¿Cuáles son la diferencias en los efectos que tiene el potencial (su magnitud y el régimen de interacción) sobre la localización de densidades que provienen de una función simétrica y de una función antisimétrica?

- ¿En qué régimen de potencial se encuentra más localizada la función antisimétrica que la simétrica? ¿Puede controlarse este orden utilizando como variable el potencial entre partículas?

- ¿Cómo afecta la indistinguibilidad a la correlación estadística entre partículas?

- ¿Cuáles partículas están más correlacionadas: las asociadas con la función antisimétrica o las asociadas con la simétrica? ¿Cómo esto depende de los potenciales del problema?

- ¿Es posible provocar que las partículas que obedecen la función simétrica estén más correlacionadas que las asociadas con la función antisimétrica utilizando un potencial entre ellas?

- ¿Cómo se relacionan la interacción y la simetría en la densidad reducida de una partícula? ¿Existe alguna manera de distinguir la densidad reducida de dos funciones, una simétrica y un antisimétrica, utilizando el potencial entre partículas? 


\subsubsection{No conmutatividad y potencial}

A continuación se plantearán las preguntas referentes a la interrelación entre las esquinas (III) y (I) del esquema de la Fig. (1.1) que pueden referirse a sistemas de una partícula bajo la influencia de un potencial de confinamiento (o paredes impenetrables), o a sistemas de dos partículas donde además del potencial de confinamiento tenemos el potencial entre partículas.

La función de Wigner $[10,11,12]$ es una representación en el espacio fase del sistema que tiene algunas propiedades importantes que se discutirán más adelante. La representación en el espacio fase supone de inmediato una discusión de las relaciones de incertidumbre y por lo tanto de los efectos de la no conmutatividad de los operadores de posición y momento.

\section{Una partícula}

- ¿Cómo influye el potencial en las relaciones de incertidumbre en modelos de una partícula confinada en una caja?

- ¿Tiene la misma influencia en las relaciones de incertidumbre un potencial repulsivo que uno atractivo?

- ¿Cómo influye el potencial (magnitud y régimen) en la localización de las funciones en el espacio fase?

- ¿Cómo influye el potencial en las regiones negativas y positivas de la función de Wigner?

\section{Dos partículas}

- ¿Cuál es el efecto del potencial de confinamiento en las relaciones de incertidumbre en los niveles de una y dos partículas del átomo de Moshinsky?

- ¿Cuál es la influencia del potencial en la correlación entre partículas en el espacio fase? 
- ¿Cómo influye el potencial (magnitud y régimen) en la localización en el espacio fase de dos partículas y en la función de Wigner reducida?

- ¿Cómo influyen los potenciales en las regiones negativas y positivas de estas funciones de Wigner?

\subsection{Efectos de la simetría de la función de onda}

El estudio de la esquina (II) del esquema de la Fig. (1.1) nos sugiere las siguientes interrogantes:

- ¿La indistinguibilidad se manifiesta de la misma manera en la correlación entre las posiciones que entre los momentos?

- ¿Cómo se manifiesta la indistinguibilidad en la localización de las partículas en el espacio de posición, en el de momento y cuáles son las diferencias?

- Sobre la densidad reducida ¿es posible distinguir la densidad que proviene de una función antisimétrica de una que proviene de una función simétrica?

\subsubsection{Indistinguibilidad y no conmutatividad}

Ahora nos referimos a la interrelación entre las esquinas (II) y (III) del esquema de la Fig. (1.1) a partir de la que podemos plantear estas preguntas:

- ¿Cómo se manifiesta el carácter simétrico o antisimétrico de la función de onda en las relaciones de incertidumbre?

- ¿Cómo afecta la simetría a la localización de la función de Wigner en el espacio fase?

- ¿Qué características tiene la función de Wigner de partículas idénticas?

- ¿De que manera afecta la (anti)simetría a la correlación entre la posición y el momento? 


\subsection{Efectos de la no conmutatividad de los oper- adores}

La no conmutatividad entre operadores provoca la relación de incertidumbre, y correlaciona la de la posición con la del momento porque debe cumplirse una cota. Así, situados en la esquina (III) del esquema de la Fig. (1.1) nos preguntamos:

- ¿Cómo medir la correlación estadística entre las variables de la posición y del momento?

Para medir esta correlación posición-momento necesitamos formular el problema con una función no separable en el espacio fase. Utilizaremos la función de Wigner por las propiedades que tiene, y sobre todo porque a partir de ella podemos obtener las marginales correctas de los espacios de posición y de momento.

En esta investigación la correlación posición-momento se estudia relacionada con la separabilidad de las funciones de Wigner de dos partículas $\left[W\left(x_{1}, p_{1}, x_{2}, p_{2}\right)\right]$ en las densidades de pares de los espacios correspondientes; y también como separabilidad de las funciones de Wigner reducidas $\left[w(x, p)=\int d x_{2} d p_{2} W\left(x, p, x_{2}, p_{2}\right)\right]$ en términos de las densidades reducidas de posición y de momento.

La relación entre la localización de la distribución en el espacio fase cuántico y el principio de incertidumbre se ha estudiado anteriormente $[13,14,15,16,17]$.

Gran parte de las preguntas planteadas en esta sección ya fueron formuladas refiriéndose a la interrelación de los potenciales y la simetría con la función de Wigner, por lo que aquí se anotarán dos cuestiones generales.

- ¿Qué información podemos extraer de las regiones negativas de la función de Wigner acerca de la localización en el espacio fase y acerca de la correlación posición-momento?

- ¿Cuál es la relación entre la localización de la función de Wigner y la localización de una función separable formada por el producto de las densidades de posición y de momento? 


\subsection{Separar las correlaciones}

Estudiar cómo la manera en que un tipo de correlación influye a los otros (por ejemplo cómo los potenciales influyen a la simetría y a la no conmutatividad) enfrenta el problema de separar tales correlaciones [18, 19]. Las correlaciones que provienen del potencial tienen un análogo en la mecánica clásica, no así las que provienen de la no-conmutatividad de los operadores y de la simetría de la función de onda, que son correlaciones que sólo están presentes en la mecánica cuántica.

En este trabajo se plantea un esquema en el que se pueden separar dos componentes de las mencionadas correlaciones mediante una manipulación algebraica: las correlaciones posición-momento y las correlaciones entre partículas (debidas a la indistinguibilidad y al potencial).

A partir de las preguntas anteriores esperamos que el lector pueda encontrar algunos elementos útiles que ilustren las relaciones entre los tres factores de correlación y de localización que se estudian. En los modelos analizados estas relaciones pueden abordarse de manera directa, no obstante aún tenemos en perspectiva hacer una indagación de ellas en sistemas atómicos y moleculares. Una perspectiva de análisis interesante puede construirse alrededor de la pregunta: ¿Cómo influye la correlación posición-momento en la formación del enlace químico?

A lo largo del trabajo se hace mención de algunas características que estos modelos comparten con sistemas atómicos y de algunas analogías conceptuales que sugieren perspectivas de estudio. 


\section{Mecánica cuántica}

\subsection{Conceptos generales}

En la mecánica cuántica la ecuación que describe la dinámica de un sistema de $N$ partículas en una dimensión es la Ecuación de Schrödinger dependiente del tiempo,

$$
\begin{aligned}
-\frac{\hbar}{i} \frac{\partial}{\partial t} \Psi\left(x_{1}, x_{2}, \ldots, x_{N}, t\right)= & -\sum_{i=1}^{N} \frac{\hbar^{2}}{2 m_{i}} \frac{\partial^{2}}{\partial x_{i}^{2}} \Psi\left(x_{1}, x_{2}, \ldots, x_{i}, \ldots, x_{N}, t\right) \\
& +V\left(x_{1}, x_{2}, \ldots, x_{N}, t\right) \Psi\left(x_{1}, x_{2}, \ldots, x_{N}, t\right) .
\end{aligned}
$$

Esta ecuación es determinista porque si conocemos la solución en el tiempo $t$ podemos utilizarla para calcular la solución (el estado en que se encontrará el sistema) en el tiempo $t+\Delta t$.

Si el potencial es independiente del tiempo podemos escribir una solución separable,

$$
\Psi\left(x_{1}, x_{2}, \ldots, x_{N}, t\right)=\psi\left(x_{1}, x_{2}, \ldots, x_{N}\right) f(t)
$$

con

$$
f(t)=e^{-i E t / \hbar}
$$

donde $E$ es la energía del sistema y aparece formalmente como la constante de separación de la ecuación diferencial, Ec. (2.1). Podemos escribir la Ecuación de Schrödinger independiente del tiempo,

$$
\begin{array}{r}
-\sum_{i=1}^{N} \frac{\hbar^{2}}{2 m_{i}} \frac{\partial^{2}}{\partial x_{i}^{2}} \psi\left(x_{1}, x_{2}, \ldots, x_{i}, \ldots, x_{N}\right)+ \\
V\left(x_{1}, x_{2}, \ldots, x_{N}\right) \psi\left(x_{1}, x_{2}, \ldots, x_{N}\right)=E \psi\left(x_{1}, x_{2}, \ldots, x_{N}\right) .
\end{array}
$$


donde

$$
\frac{\hat{p}_{i}^{2}}{2 m_{i}}=-\frac{\hbar^{2}}{2 m_{i}} \frac{\partial^{2}}{\partial x_{i}^{2}}
$$

es el operador de la energía cinética de la $i$-ésima partícula. Es un operador de una sola variable (monoelectrónico en el caso de electrones). Su forma se debe a que la representación del operador $\hat{p}$ en el espacio de posición es

$$
\hat{p} \equiv-i \hbar \frac{\partial}{\partial x}
$$

este operador es el responsable de los desplazamientos en el espacio real. Mediante la suma en la Ec. (2.3) sobre todas las partículas obtenemos la energía cinética del sistema.

Por otro lado,

$$
\hat{V}\left(x_{1}, x_{2}, \ldots, x_{N}\right)=V\left(x_{1}, x_{2}, \ldots, x_{N}\right)
$$

es el operador de energía potencial. Es un operador de muchos cuerpos. Se puede ver fácilmente a partir de la forma de la Ec. (2.3) que se debe a este potencial que no se pueda escribir una solución separable a menos que,

$$
V\left(x_{1}, x_{2}, \ldots, x_{N}\right)=\sum_{i} V\left(x_{i}\right)
$$

o que $V\left(x_{1}, x_{2}, \ldots, x_{N}\right)=$ cte, donde $V=0$ es un caso especial importante. En los casos mencionados podemos separar el problema,

$$
\psi\left(x_{1}, x_{2}, \ldots, x_{N}\right)=\phi_{1}\left(x_{1}\right) \phi_{2}\left(x_{2}\right) \ldots \phi_{N}\left(x_{N}\right)
$$

sin embargo, para respetar la indistinguibilidad de las partículas, tenemos que simetrizar la solución escribiendo una función determinantal de tipo Slater,

$$
\left|\phi_{1}\left(x_{1}\right) \phi_{2}\left(x_{2}\right) \ldots \phi_{N}\left(x_{N}\right)\right|
$$

o un permanente,

$$
\left|\phi_{1}\left(x_{1}\right) \phi_{2}\left(x_{2}\right) \ldots \phi_{N}\left(x_{N}\right)\right|_{+} .
$$


Cabe señalar que la indistinguibilidad es un postulado de la mecánica cuántica no relativista que implica,

$$
\psi\left(x_{1}, x_{2}, \ldots, x_{i}, \ldots, x_{j}, \ldots, x_{N}\right)= \pm \psi\left(x_{1}, x_{2}, \ldots, x_{j}, \ldots, x_{i}, \ldots, x_{N}\right)
$$

condición que se satisface porque es característica de los determinantes que

$$
\left|\phi_{1}\left(x_{1}\right) \ldots \phi_{i}\left(x_{i}\right) \ldots \phi_{j}\left(x_{j}\right) \ldots \phi_{N}\left(x_{N}\right)\right|=-\left|\phi_{1}\left(x_{1}\right) \ldots \phi_{i}\left(x_{j}\right) \ldots \phi_{j}\left(x_{i}\right) \ldots \phi_{N}\left(x_{N}\right)\right|
$$

y de los permanentes,

$$
\left|\phi_{1}\left(x_{1}\right) \ldots \phi_{i}\left(x_{i}\right) \ldots \phi_{j}\left(x_{j}\right) \ldots \phi_{N}\left(x_{N}\right)\right|_{+}=\left|\phi_{1}\left(x_{1}\right) \ldots \phi_{i}\left(x_{j}\right) \ldots \phi_{j}\left(x_{i}\right) \ldots \phi_{N}\left(x_{N}\right)\right|_{+} .
$$

Las funciones antisimétricas se caracterizan por tener el hueco de Fermi que en el caso sin espín es la imposibilidad de encontrar dos partículas con las mismas coordenadas espaciales. El hueco de Fermi significa que,

$$
\psi\left(x_{1}, x_{2}, \ldots, x, \ldots, x, \ldots, x_{N}\right)=0
$$

Por otro lado, cuando el potencial no permite escribir una solución separable, se sabe que basta con incluir las interacciones por pares para que los sistemas sean bien descritos energéticamente. Así, lo frecuente es considerar que es un operador de dos cuerpos

$$
V\left(x_{1}, x_{2}, \ldots, x_{N}\right)=\sum_{i \neq j}^{N} V\left(x_{i}, x_{j}\right)
$$

Un caso especial pero muy frecuente se da cuando la única variable es la distancia entre pares de partículas, entonces,

$$
V\left(x_{1}, x_{2}, \ldots, x_{N}\right)=\sum_{i \neq j}^{N} V\left(x_{i}-x_{j}\right),
$$

como el caso de los potenciales coulómbicos en los que hablamos del hueco de Coulomb para referirnos al hecho de que dos partículas con la misma carga se evitan debido a la repulsión, la cual aumenta conforme disminuye la distancia entre partículas, llegando a ser infinita en la singularidad del potencial cuando $x_{i}-x_{j}=0$. 
Como se deriva de la discusión anterior, en general, la función de onda no es separable en un producto de funciones independientes debido al potencial y debido a la simetrización.

Por otro lado, la norma cuadrada de la función de onda,

$$
\left|\psi\left(x_{1}, x_{2}, \ldots, x_{i}, \ldots, x_{j}, \ldots, x_{N}\right)\right|^{2}
$$

es la densidad de probabilidad y está relacionada con la probabilidad de encontrar la partícula 1 en $x_{1}+d x_{1}$, simultáneamente con la 2 en $x_{2}+d x_{2} \ldots$ y la $N$-ésima en $x_{N}+d x_{N}$, dicha probabilidad es,

$$
\left|\psi\left(x_{1}, x_{2}, \ldots, x_{N}\right)\right|^{2} d x_{1} d x_{2} \ldots d x_{N} .
$$

Sobre esta base pueden definirse diferentes densidades de probabilidad reducida integrando la densidad de probabilidad sobre las partículas convenientes. Si integramos sobre todas las partículas excepto una tenemos $\rho(x)$,

$$
\rho(x)=\int d x_{2} \ldots d x_{N}\left|\psi\left(x, x_{2}, \ldots, x_{N}\right)\right|^{2},
$$

que es la densidad de probabilidad de encontrar una partícula, sin importar cual, en una región del espacio.

El valor de la función $\left|\psi\left(x_{1}, x_{2}, \ldots, x_{N}\right)\right|^{2}$ con valores de $x_{i}$ 's similares entre sí nos habla de la probabilidad de encontrar a las partículas concentradas en una región del espacio, localizadas, lo que puede interpretarse como una condensación. Debido al hueco de Fermi se espera comportamientos diferentes de las funciones simétricas respecto de las antisimétricas. Así, podría también intentarse una caracterización de diferentes fases del sistema analizando las probabilidades de encontrar las partículas en cierta región o deslocalizadas en todo el espacio.

Hemos escrito las ecuaciones en la representación del espacio de posiciones pero también podemos hacerlo en el espacio de momento, la representación del operador $\hat{x}$ en ese espacio es

$$
\hat{x}_{m o m} \equiv-i \hbar \frac{\partial}{\partial p}
$$


en tanto que la representación del momento es $\hat{p} \equiv p$, y la solución en ese espacio nos daría la función de onda en el espacio de momento,

$$
\Phi\left(p_{1}, p_{2}, \ldots, p_{i}, \ldots, p_{j}, \ldots, p_{N}\right)
$$

cuya norma cuadrada es la densidad de probabilidad de momentos y multiplicada por el elemento de volumen se interpreta como la probabilidad de que la partícula 1 tenga momento en $p_{1}+d p_{1}$, simultáneamente con que el momento de la 2 en $p_{2}+d p_{2} \ldots$ y el de la $N$-ésima en $p_{N}+d p_{N}$,

$$
\left|\Phi\left(p_{1}, p_{2}, \ldots, p_{N}\right)\right|^{2} d p_{1} d p_{2} \ldots d p_{N}
$$

Si la distribución está localizada indicaría que las partículas tienen momento similar. También la dispersión de las distribuciones y su localización podrían hablar de fases diferentes del sistema. Si pensamos en el valor esperado del momento, $\langle p\rangle$, éste puede ser cero debido a simetrías en el sistema ${ }^{1}$. Así, $\langle p\rangle=0$ no implica que las partículas estén en reposo. Nos importa este punto porque la medida de la incertidumbre usual, la desviación estándar, mide la desviación de la distribución respecto del valor esperado ${ }^{2}$, y en casos en que no se trata de gaussianas la localización que se mide de esta forma es aproximada.

Se postula que cada una de las funciones de onda (en posición y en momento) tiene toda la información del estado del sistema. Ambas funciones de onda, además de ser soluciones de las ecuaciones de Schrödinger en el espacio respectivo, están relacionadas por la Transformada de Dirac-Fourier [20, 21],

$$
\Phi\left(p_{1}, \ldots, p_{N}\right)=\frac{1}{\sqrt{2 \pi \hbar}} \int d x_{1} \ldots d x_{N} \psi\left(x_{1}, \ldots, x_{N}\right) e^{-i\left(p_{1} x_{1}+\ldots+p_{N} x_{N}\right) / \hbar}
$$

\footnotetext{
${ }^{1}$ En muchos sistemas el valor esperado es cero $(\langle\hat{p}\rangle=0)$ debido a que la probabilidad de que la velocidad sea positiva o negativa es igual para cada punto de $x$. El momento es una cantidad vectorial y esto se refleja de esta manera en los escalares.

${ }^{2}$ La definición es $\sqrt{\left\langle p^{2}\right\rangle-\langle p\rangle^{2}}$. Más adelante profundizaremos en esto.
} 


\subsection{El Principio de Incertidumbre}

La relación en la Ec. (2.5) entre los espacios es consecuencia de que los operadores de posición y momento no conmutan, su conmutador es

$$
\left[\hat{x}, \hat{p_{x}}\right]=\hat{x} \hat{p_{x}}-\hat{p_{x}} \hat{x}=i \hbar
$$

La no conmutatividad de estos operadores también provoca una relación entre sus incertidumbres, el llamado principio de indeterminación de Heisenberg [22], que usualmente se escribe como,

$$
\Delta x \Delta p_{x} \geq \frac{\hbar}{2}
$$

$\operatorname{con} \Delta x$ y $\Delta p_{x}$

$$
\Delta x=\sqrt{\left\langle x^{2}\right\rangle-\langle x\rangle^{2}}, \quad \Delta p_{x}=\sqrt{\left\langle p_{x}^{2}\right\rangle-\left\langle p_{x}\right\rangle^{2}}
$$

las desviaciones estándar de la posición y del momento respectivamente. Puede verse que la desviación estándar es una medida de la desviación de los valores que la variable puede asumir de acuerdo a su distribución con respecto a un punto específico de la misma, es decir, valor esperado de la variable correspondiente, $\langle\hat{x}\rangle$ ó $\left\langle\hat{p_{x}}\right\rangle$.

Siguiendo a Robertson [23], puede escribirse el principio de incertidumbre de la forma,

$$
\Delta x \Delta p_{x} \geq \frac{1}{2}\left|\left\langle\left[\hat{x}, \hat{p}_{x}\right]\right\rangle\right|=\left|\frac{\left\langle\hat{x} \hat{p}_{x}-\hat{p}_{x} \hat{x}\right\rangle}{2}\right|=\frac{1}{2} \int d x \Psi^{*}(x)\left\{\hat{x} \hat{p}_{x}-\hat{p}_{x} \hat{x}\right\} \Psi(x)
$$

La forma más general de escribir el principio de incertidumbre utilizando las desviaciones estándar es la de Schrödinger [24],

$$
(\Delta x)^{2}\left(\Delta p_{x}\right)^{2} \geq\left(\frac{\left\langle\hat{x} \hat{p}_{x}+\hat{p}_{x} \hat{x}\right\rangle}{2}-\langle\hat{x}\rangle\left\langle\hat{p}_{x}\right\rangle\right)^{2}+\left|\frac{\left\langle\hat{x} \hat{p}_{x}-\hat{p}_{x} \hat{x}\right\rangle}{2}\right|^{2} .
$$

El primer término del lado derecho de la desigualdad es la covarianza cuántica entre $\hat{x}$ y $\hat{p}_{x}, \operatorname{cov}\left(\hat{x}, \hat{p}_{x}\right)$, elevada al cuadrado. Destacaremos algunos puntos tomando en cuenta esta ecuación: 
- La cota es más estricta que la de la Ec. (2.9). Si, $\operatorname{cov}\left(\hat{x}, \hat{p}_{x}\right)=0$, ambas cotas son iguales.

- El principio de incertidumbre puede relacionarse con la correlación estadística existente entre los operadores $\hat{x}$ y $\hat{p}_{x}$ medida en este caso con $\operatorname{cov}\left(\hat{x}, \hat{p}_{x}\right)$.

- La correlación entre incertidumbres involucra la correlación entre operadores y por lo tanto entre las variables que los representan.

- Si $\Psi$ es una función real, $\operatorname{cov}\left(\hat{x}, \hat{p}_{x}\right)=0$ [25], por lo tanto la correlación entre posición y momento debe estar relacionada con la fase de la función de onda.

- $\operatorname{cov}\left(\hat{x}, \hat{p}_{x}\right)=0$ no implica que no exista correlación entre posición y momento, simplemente que la covarianza no puede detectarla ${ }^{3}$.

Los estados que cumplen con la cota inferior del Principio de Incertidumbre son llamados "estados de mínima incertidumbre" o coherentes, este es el caso de las funciones gaussianas, soluciones del estado basal del oscilador armónico.

Si quisiéramos estudiar el principio de incertidumbre en distribuciones de dos partículas deberíamos introducir una desviación estándar multidimensional, para lo cual se puede utilizar la matriz de covarianzas, pero su interpretación no es tan clara como en el caso unidimensional, es decir, no es una simple generalización. En nuestra investigación no hemos encontrado una discusión del principio de incertidumbre de Heisenberg de dos partículas.

En contraste, en la teoría de la información existe una definición de un principio de incertidumbre para dos partículas, que es una generalización físicamente transparente del de una partícula.

\footnotetext{
${ }^{3}$ La covarianza es capaz de detectar solamente correlaciones lineales.
} 


\subsection{El átomo de Moshinsky}

El átomo de Moshinsky es un modelo de dos partículas interactuando a través de un potencial armónico y que se encuentran atrapadas en una trampa armónica [26, 27]. Puede interpretarse como modelando un átomo de Helio, con los potenciales de Coulomb sustituidos por potenciales armónicos, por esta analogía es que se suele llamar "átomo" al modelo de Moshinsky. La versión unidimensional [28] del Hamiltoniano del sistema es,

$$
\hat{H}=\frac{\hat{p}_{1}^{2}}{2 m}+\frac{\hat{p}_{2}^{2}}{2 m}+\frac{1}{2} \omega^{2} m \hat{x}_{1}^{2}+\frac{1}{2} \omega^{2} m \hat{x}_{2}^{2} \pm \frac{1}{2} \lambda^{2} m\left(\hat{x}_{1}-\hat{x}_{2}\right)^{2}
$$

Los subíndices etiquetan a las partículas, $\omega$ es la frecuencia de la trampa armónica, $m$ es la masa de los osciladores (que tomaremos $m=1$ ) y $\lambda$ es la frecuencia de la interacción armónica entre las partículas. El signo $(+)$ se corresponde con un potencial atractivo, y (-) corresponde a un potencial repulsivo. Este Hamiltoniano puede resolverse analíticamente si se escribe en términos de las coordenadas del centro de masa y de las relativas que respectivamente se definen por,

$$
\hat{R}=\frac{\hat{x}_{1}+\hat{x}_{2}}{\sqrt{2}}, \quad \hat{r}=\frac{\hat{x}_{1}-\hat{x}_{2}}{\sqrt{2}}
$$

y los momentos correspondientes,

$$
\hat{P}=\frac{\hat{p}_{1}+\hat{p}_{2}}{\sqrt{2}}, \quad \hat{p}=\frac{\hat{p}_{1}-\hat{p}_{2}}{\sqrt{2}}
$$

donde $(\operatorname{con} \hbar=1)$

$$
\hat{H}=\hat{H}_{R}+\hat{H}_{r}=\frac{1}{2}\left(\hat{P}^{2}+\omega^{2} \hat{R}^{2}\right)+\frac{1}{2}\left(\hat{p}^{2}+\Lambda^{2} \hat{r}^{2}\right) .
$$

El nuevo potencial asociado con las coordenadas relativas es

$$
\Lambda=\sqrt{\omega^{2} \pm 2 \lambda^{2}}=\omega \sqrt{1 \pm 2 \frac{\lambda^{2}}{\omega^{2}}}
$$

con la misma interpretación de los signos que se presentó antes. Puede verse que $\omega \rightarrow \infty$ es el caso límite no interactuante. Para un potencial atractivo, $\omega \in(0, \infty)$, y 
para un potencial repulsivo, $\omega \in(\sqrt{2} \lambda, \infty)$, para que $\Lambda$ sea real. $\omega \rightarrow 0$ y $\omega \rightarrow \sqrt{2} \lambda$ son los casos límites de partículas interactuantes sin confinamiento en una trampa en los regímenes atractivo y repulsivo, respectivamente.

La energía de los estados está dada por,

$$
E_{n_{R}, n_{r}}(\omega, \lambda)=\omega\left(n_{R}+\frac{1}{2}\right)+\sqrt{\omega^{2} \pm 2 \lambda^{2}}\left(n_{r}+\frac{1}{2}\right) .
$$

Las soluciones en el espacio de posición son [29],

$$
\psi_{n_{R}}(R)=\left(\frac{\omega^{1 / 2}}{2^{n_{R}} n_{R} ! \pi^{1 / 2}}\right)^{1 / 2} e^{-\omega R^{2} / 2} H_{n_{R}}(\sqrt{\omega} R)
$$

$\mathrm{y}$

$$
\psi_{n_{r}}(r)=\left(\frac{\Lambda^{1 / 2}}{2^{n_{r}} n_{r} ! \pi^{1 / 2}}\right)^{1 / 2} e^{-\Lambda r^{2} / 2} H_{n_{r}}(\sqrt{\Lambda} r)
$$

Por lo tanto,

$$
\Psi\left(x_{1}, x_{2}\right)=\psi_{n_{R}}(R) \psi_{n_{r}}(r) .
$$

Por otra parte, si escribimos el Hamiltoniano en la representación de momento conseguimos las soluciones,

$$
\phi_{n_{R}}(P)=\left(\frac{1}{2^{n_{R}} n_{R} ! \omega^{1 / 2} \pi^{1 / 2}}\right)^{1 / 2} e^{-P^{2} / 2 \omega} H_{n_{R}}(P / \sqrt{\omega})
$$

$\mathrm{y}$

$$
\phi_{n_{r}}(p)=\left(\frac{1}{2^{n_{r}} n_{r} ! \Lambda^{1 / 2} \pi^{1 / 2}}\right)^{1 / 2} e^{-p^{2} / 2 \Lambda} H_{n_{r}}(p / \sqrt{\Lambda}) .
$$

Entonces,

$$
\Phi\left(p_{1}, p_{2}\right)=\phi_{n_{R}}(P) \phi_{n_{r}}(p)
$$

Las etiquetas $n_{R}$ y $n_{r}$ corresponden a los números cuánticos asociados con el centro de masa y las coordenadas relativas respectivamente, y $H_{n}(x)$ son los polinomios de Hermite de $n$-ésimo orden. Las soluciones no son separables en términos de $x_{1}$ y $x_{2}$ ó $p_{1}$ y $p_{2}$, por lo que existe correlación estadística entre las variables originales.

La noción de entrelazado depende de las coordenadas en que se trata y resuelve el sistema. Por ejemplo, en este caso existe entrelazado entre las variables originales pero no en las coordenadas del centro de masa y las coordenadas relativas. 
El átomo de Moshinsky se ha estudiado extensamente en muchos contextos, tales como la teoría de la información [30, 31, 32, 33, 34], correlaciones cuánticas y entrelazado [34, 29, 35, 36, 37, 38, 39], espacio fase de Wigner [38, 39, 40, 28, 41], matrices de la densidad y teoría de funcionales de la densidad [28, 41, 42, 43, 44, 45, 46, 47, 48, 49, 50], funciones de distribución de pares [32, 51, 52, 53], energía de correlación $[30,38,54,55,56]$ y otros contextos $[57,58,59]$. Para enfatizar la importancia del modelo señalamos que puede reproducir comportamientos generales de sistemas atómicos realistas [37].

\subsection{Una variación en el modelo}

En el átomo de Moshinsky tenemos un régimen de interacción atractivo y otro repulsivo según el signo del potencial, $\lambda$. Para ambos el límite no interactuante es el mismo, $\omega \rightarrow \infty$, pero el límite de no confinamiento es distinto.

Tenemos interés en estudiar cambios en las propiedades cuando pasamos del régimen atractivo al repulsivo por lo que debemos formular el Hamiltoniano de manera tal que permita ir de un régimen a otro de manera continua. Para ello definimos un parámetro que controle la interacción entre partículas como $\kappa=\lambda / \omega$, y tenemos $(\operatorname{con} m=1$ y $\hbar=1)$

$$
\hat{H}=\frac{1}{2} \hat{p}_{1}^{2}+\frac{1}{2} \hat{p}_{2}^{2}+\frac{1}{2} \omega^{2} \hat{x}_{1}^{2}+\frac{1}{2} \omega^{2} \hat{x}_{2}^{2}+\frac{1}{2} \omega^{2} \kappa\left(\hat{x}_{1}-\hat{x}_{2}\right)^{2} .
$$

Hemos escrito el potencial entre partículas en las unidades del potencial de confinamiento, $\omega$. Expresando el Hamiltoniano en términos de las coordenadas del centro de masa y relativas: $\hat{H}=\hat{H}_{c m}+\hat{H}_{r e l}$

$$
\hat{H}_{c m}=\frac{\hat{P}^{2}}{2}+\frac{1}{2} \omega^{2} \hat{R}^{2}, \quad \hat{H}_{r e l}=\frac{\hat{p}^{2}}{2}+\frac{1}{2} \Lambda^{2} \hat{r}^{2}
$$

donde resulta el potencial asociado a las coordenadas relativas,

$$
\Lambda=\omega \sqrt{2 \kappa+1}
$$


$\kappa$ es el parámetro que controla la intensidad de la interacción entre las partículas,

$$
-\frac{1}{2}<\kappa<\infty
$$

para que $\Lambda$ permanezca real. La interacción es repulsiva para $\kappa<0$ y es atractiva para $\kappa>0, \kappa=0$ es el caso de no interacción. Podemos entonces ir de un régimen de interacción al otro de manera continua.

A lo largo de este trabajo estudiaremos la interacción entre partículas valiéndonos de $\kappa$ o de $\lambda$ dependiendo del tipo de efectos a analizar. 


\section{Función de Wigner}

\subsection{La representación de Dirac}

Podemos escribir las ecuaciones de la mecánica cuántica en la representación de Dirac, lo cual es más general que escribirlas en la representación de posición o de momento. En esta representación la ecuación de Schrödinger se lee

$$
i \hbar \frac{\partial}{\partial t}|\psi(t)\rangle=\hat{H}|\psi(t)\rangle
$$

donde el símbolo $|\psi\rangle$ representa el vector de estado que simboliza un estado cuántico y es llamado un ket. Las funciones de onda de posición y momento, respectivamente, se escriben es esta representación como,

$$
\psi(x, t)=\langle x \mid \psi(t)\rangle, \quad \phi(p, t)=\langle p \mid \phi(t)\rangle .
$$

Para representar el estado los kets deben estar normalizados,

$$
\langle\psi \mid \psi\rangle=1
$$

donde $\langle\psi|$ es llamado un bra y se encuentra en correspondencia uno a uno con el ket, cumpliendo $\langle\psi|=| \psi\rangle^{*}$ y el producto escalar tiene usualmente la propiedad,

$$
\langle\phi \mid \psi\rangle=\langle\psi \mid \phi\rangle^{*}
$$

la cantidad $\langle\phi \mid \psi\rangle$ es la amplitud de probabilidad de observar al sistema en el estado $|\phi\rangle$, dado que sabemos que se encuentra en el estado $|\psi\rangle$. 
El espacio vectorial al cual pertenecen los vectores $|\psi\rangle$ es conocido como el espacio de Dirac-Hilbert. En este espacio podemos elegir bases continuas, discretas, o una combinación para representar el vector de estado. Podemos elegir bases ortonormales, en el caso de bases discretas esta condición significa que,

$$
\left\langle\psi_{i} \mid \psi_{j}\right\rangle=\delta_{i j}
$$

y en el caso continuo,

$$
\langle\alpha \mid \alpha \prime\rangle=\delta(\alpha-\alpha \prime)
$$

donde $\delta$ es la delta de Dirac, y $\alpha$ podría representar la posición $(x)$ o el momento $(p)$.

Un operador $\hat{A}$ es representado en la notación de Dirac por una matriz, $\left\langle\psi_{i}|\hat{A}| \psi_{j}\right\rangle$ en el caso discreto y $\langle\alpha|\hat{A}| \alpha \prime\rangle$ en el continuo.

Se dice la elección de la base en el espacio de Dirac-Hilbert define una representación. La base de vectores $|x\rangle$ define la representación de posición, $|p\rangle$ la representación de momento, etcétera.

\subsection{El operador de la densidad}

En lugar de representar un estado mecano-cuántico por medio de un vector de estado normalizado, $|\psi\rangle^{1}$, podemos representarlo por medio del operador,

$$
\hat{\rho}=|\psi\rangle\langle\psi|
$$

cuyo valor esperado es,

$$
\langle\hat{\rho}\rangle=\langle\psi \mid \psi\rangle\langle\psi \mid \psi\rangle=1
$$

El operador de la densidad permite calcular los valores esperados de los operadores mediante la ecuación,

$$
\langle\hat{A}\rangle=\operatorname{Tr}(\hat{\rho} \hat{A})=\operatorname{Tr}(\hat{A} \hat{\rho}),
$$

y la diagonal de este operador en las representaciones correspondientes produce las densidades de probabilidad, en el espacio de posición,

\footnotetext{
${ }^{1}$ Que escribimos independiente del tiempo.
} 


$$
\langle x|\hat{\rho}| x\rangle=\langle x \mid \psi\rangle\langle\psi \mid x\rangle=\psi(x) \psi(x)^{*}=\rho(x)
$$

y en el de momento,

$$
\langle p|\hat{\rho}| p\rangle=\langle p \mid \psi\rangle\langle\psi \mid p\rangle=\psi(p) \psi(p)^{*}=\pi(p)
$$

que hemos escrito para una partícula en 1D pero puede generalizarse al caso de varias partículas y/o varias dimensiones.

El operador de la densidad mecano-cuántico guarda una analogía con la densidad clásica en espacio fase, en el sentido de que pueden usarse de manera muy similar. También, el operador de la densidad podemos representarlo en el espacio fase, si escribimos,

$$
W(x, p)=\frac{1}{\pi \hbar} \int d y\langle x+y \mid \psi\rangle\langle\psi \mid x-y\rangle e^{-2 i p y / \hbar}
$$

$\mathrm{O}$

$$
W(x, p)=\frac{1}{\pi \hbar} \int d y \psi(x-y)^{*} \psi(x+y) e^{-2 i p y / \hbar}
$$

$W(x, p)$ es la función de Wigner, introducida en 1932 por E. P. Wigner [10]. Todas las integrales en este capítulo se realizan en el intervalo $(-\infty, \infty)$, a menos que se indique otra cosa. Una forma equivalente en términos de la función de onda en el espacio de momentos es,

$$
W(x, p)=\frac{1}{\pi \hbar} \int d u \phi(p-u)^{*} \phi(p+u) e^{2 i x u / \hbar}
$$

La función de Wigner es entonces una representación del operador de la densidad y puede utilizarse formalmente como una densidad de probabilidad con la que pueden calcularse valores esperados de una forma totalmente análoga a la mecánica clásica,

$$
\langle A(\hat{x}, \hat{p})\rangle=\int d x d p a(x, p) W(x, p)
$$

donde $a(x, p)$ se obtiene sustituyendo los operadores por variables en $A(\hat{x}, \hat{p})$. 


\subsection{Propiedades de la función de Wigner}

Las propiedades más notables de la función de Wigner son que sus marginales son las densidades de posición y momento que se obtendrían en el formalismo de Schrödinger, y por lo tanto la función está normalizada. Para derivar estas propiedades seguiremos el tratamiento que realiza Dahl [60].

Sea $\psi(x)$ la función de onda normalizada de un estado cuántico en el espacio de posición y $\phi(p)$ la función de onda en el espacio de momento. Como se ha anotado en el capítulo anterior, ambas funciones se encuentran conectadas por medio de la transformada de Dirac-Fourier,

$$
\begin{gathered}
\psi(x)=\frac{1}{\sqrt{2 \pi \hbar}} \int d p \phi(p) e^{i p x / \hbar} \\
\phi(p)=\frac{1}{\sqrt{2 \pi \hbar}} \int d x \psi(x) e^{-i p x / \hbar} .
\end{gathered}
$$

Las cantidades

$$
\psi_{i}(x)^{*} \psi_{j}(x)
$$

y

$$
\phi_{i}(p)^{*} \phi_{j}(p)
$$

representan densidades de probabilidad en los espacios de posición y momento, respectivamente, cuando $i$ y $j$ refieren al mismo estado $(i=j)$ y densidades de transición cuando $i$ y $j$ refieren a estados diferentes $(i \neq j)$. Sustituyendo la Ec. (3.16) en la Ec. (3.18) para $\psi_{j}(x)$ y la Ec. (3.17) en la Ec. (3.19) para $\phi_{i}(p)^{*}$, obtenemos,

$$
\psi_{i}(x)^{*} \psi_{j}(x)=\frac{1}{\sqrt{2 \pi \hbar}} \int d p \psi_{i}(x)^{*} \phi_{j}(p) e^{i p x / \hbar}
$$

y

$$
\phi_{i}(p)^{*} \phi_{j}(p)=\frac{1}{\sqrt{2 \pi \hbar}} \int d x \psi_{i}(x)^{*} \phi_{j}(p) e^{i p x / \hbar}
$$

Estas expresiones muestran que la distribución en espacio fase

$$
f_{i j}^{0}(x, p)=\frac{1}{\sqrt{2 \pi \hbar}} \psi_{i}(x)^{*} \phi_{j}(p) e^{i p x / \hbar}
$$


tiene las funciones $\psi_{i}(x)^{*} \psi_{j}(x)$ y $\phi_{i}(p)^{*} \phi_{j}(p)$ como densidades marginales. Por lo tanto podemos considerar a $f_{i j}^{0}$ formalmente como una densidad en espacio fase.

Sustituyendo la Ec. (3.17) por $\phi_{j}(p)$ en la Ec. (3.22), obtenemos la expresión,

$$
f_{i j}^{0}(x, p)=\frac{1}{2 \pi \hbar} \int d x^{\prime} \psi_{i}(x)^{*} \psi_{j}\left(x^{\prime}\right) e^{i p\left(x-x^{\prime}\right) / \hbar} .
$$

Alternativamente, podemos definir $x^{\prime}$ en términos de $x$ valiéndonos de otra variable, $y$. Así, haciendo el cambio de variable $x^{\prime}=x+2 y$, tenemos $d x^{\prime}=2 d y$, la integral es sobre $y$ porque $x$ es una constante en la integral de la Ec. $(3.23)^{2}$,

$$
f_{i j}^{0}(x, p)=\frac{1}{\pi \hbar} \int d y \psi_{i}(x)^{*} \psi_{j}(x+2 y) e^{-2 i p y / \hbar}
$$

pero $f_{i j}^{0}(x, p)$ es una función compleja aún para $i=j$. Una función está evaluada en $x$ y la otra en una distancia a $2 y$ de ésta.

Podemos cambiar los argumentos de las funciones manteniendo que la distancia que separa una función de la otra es $2 y$, mediante la sustitución $(x \rightarrow x-y)$. Cambiamos así la fase en la integral y conseguimos la función,

$$
f_{i j}^{W}(x, p)=\frac{1}{\pi \hbar} \int d y \psi_{i}(x-y)^{*} \psi_{j}(x+y) e^{-2 i p y / \hbar}=W(x, p)
$$

que tiene las mismas marginales que $f_{i j}^{0}(x, p)$ y es real para $i=j$, esta es la función de Wigner.

Vamos a discutir brevemente por qué la función de Wigner es real. Primero dividamos la integral en la Ec. (3.25) alrededor de cero,

$$
\begin{aligned}
W(x, p) & =\frac{1}{\pi \hbar} \int_{-\infty}^{0} d y \psi_{i}(x-y)^{*} \psi_{j}(x+y) e^{-2 i p y / \hbar} \\
& +\frac{1}{\pi \hbar} \int_{0}^{\infty} d y \psi_{i}(x-y)^{*} \psi_{j}(x+y) e^{-2 i p y / \hbar} .
\end{aligned}
$$

La variable de integración es muda, por tanto en la primera integral podemos sustituir, $y \rightarrow-y$, obtenemos,

$$
\begin{aligned}
W(x, p)= & -\frac{1}{\pi \hbar} \int_{\infty}^{0} d y \psi_{i}(x+y)^{*} \psi_{j}(x-y) e^{2 i p y / \hbar} \\
& +\frac{1}{\pi \hbar} \int_{0}^{\infty} d y \psi_{i}(x-y)^{*} \psi_{j}(x+y) e^{-2 i p y / \hbar}
\end{aligned}
$$

\footnotetext{
${ }^{2}$ Podemos utilizar $x+y$, en lugar de $x+2 y$.
} 
En esa misma integral invertimos los límites de integración,

$$
\begin{aligned}
W(x, p) & =\frac{1}{\pi \hbar} \int_{0}^{\infty} d y \psi_{i}(x+y)^{*} \psi_{j}(x-y) e^{2 i p y / \hbar} \\
& +\frac{1}{\pi \hbar} \int_{0}^{\infty} d y \psi_{i}(x-y)^{*} \psi_{j}(x+y) e^{-2 i p y / \hbar}
\end{aligned}
$$

Ahora, notemos que los límites de integración son los mismos por lo que podemos sumar los integrandos. El integrando de la primera integral es el complejo conjugado del integrando en la segunda integral. La suma de un número complejo y su complejo conjugado es real,

$$
a+a^{*}=(x+i y)+(x-i y)=2 x .
$$

por lo tanto la función definida en la Ec. (3.25) es real.

Ahora nos referiremos a la negatividad de la función de Wigner. Podemos escribir el traslape de dos funciones de Wigner correspondientes a los estados $\psi(x)$ y $\xi(x)$,

$$
\left|\int d x \psi^{*}(x) \xi(x)\right|^{2}=(2 \pi \hbar) \int d x d p W_{\psi}(x, p) W_{\xi}(x, p) .
$$

Si $\psi(x)=\xi(x)$,

$$
\int d x d p W(x, p)^{2}=\frac{1}{2 \pi \hbar}
$$

lo que excluye distribuciones muy localizadas del tipo [11],

$$
P_{\psi}(x, p)=\delta\left(x-x^{\prime}\right) \delta\left(p-p^{\prime}\right)
$$

donde $\delta$ es la función delta de Dirac.

Si $\psi(x)$ y $\xi(x)$ son ortogonales,

$$
\int d x d p W_{\psi}(x, p) W_{\xi}(x, p)=0
$$

que implica que la función de Wigner no es positivo-definida, de hecho sólo es positiva en todo el espacio para funciones gaussianas [11, 12] como el estado basal del oscilador armónico. Ambos resultados para el traslape de la función de Wigner son consecuencia 
de que debe respetar el principio de incertidumbre. Debido a la negatividad a la función de Wigner se le llama una densidad de "cuasiprobabilidad" o una "cuasidensidad".

Además, los valores de la función de Wigner de un estado puro se encuentran acotados [12],

$$
-\frac{1}{\pi \hbar} \leq W(x, p) \leq \frac{1}{\pi \hbar}
$$

En unidades atómicas $(\hbar=1)$ la cota es, $1 / \pi \approx 0.318$. Esta es una consecuencia también del principio de incertidumbre pues prohibe funciones de Wigner muy localizadas. Queda manifiesta una relación entre localización en el espacio fase y el principio de incertidumbre que ha sido explorada en la literatura [16, 17, 13, 14, 15, 61, 62, 63, 64]. Cabe señalar que la función de Wigner puede ser reconstruida a partir de datos experimentales $[65,66]$.

La función de Wigner se utiliza en muchos campos de la física, particularmente en óptica. Sin embargo, el interés en la función de Wigner para estudiar sistemas atómicos $[67,68,69,70,71]$ y moleculares $[72,73]$ ha aumentado en los últimos años.

Especialmente nos interesa mencionar que ha encontrado aplicación en la Teoría de Funcionales de la Densidad, donde existe una dificultad en la evaluación de las cantidades relacionadas naturalmente con el espacio de momento, tales como la energía cinética, debido a que dependen en los elementos no diagonales de la matriz de la densidad. Considerando la función de Wigner y no suprimiendo la dependencia en los momentos se esperaría nuevas vías para el diseño y cálculo de funcionales de la densidad que son difíciles de aproximar con la densidad en el espacio de posición [28, 41, 50]. 


\section{Teoría de la Información}

La Teoría de la Información se establece sobre la base de una serie de axiomas que debe cumplir una medida cuantitativa de la información: la entropía de Shannon; actualmente se le reconoce como una parte de la teoría estadística, por lo que sus aplicaciones no están limitadas a los casos en los que la información en su sentido comunicativo es importante. En el marco de la teoría pueden definirse una medida de la incertidumbre en una distribución (la entropía de Shannon) y una medida de correlación estadística entre variables (la información mutua), las cuales son alternativas a la desviación estándar y al coeficiente de correlación respectivamente. Los conceptos de localización y de correlación son importantes en muchas disciplinas científicas.

El trabajo de Shannon de 1948, "A mathematical theory of communication" [74], aborda el problema de la información en su sentido comunicativo, lo cual dirigió, casi exclusivamente, la teoría por algunos años. Medir la información es también medir la incertidumbre y ésta no es propia de los problemas comunicacionales sino de cualquier problema estadístico. Sus aplicaciones son extensas en los procesos de transmisión de información, porque ofrece una medida cuantitativa de la información y también es una medida del ruido, del ancho del canal de transmisión, etcétera. Además, la teoría de la información provee de métodos por los cuales puede tratarse el ruido y otros problemas en ese campo.

Como parte de la teoría estadística, sus aplicaciones se extienden a todas las ramas de la ciencia donde el análisis estadístico es importante. La mecánica cuántica es una teoría estadística que nos permite calcular densidades de probabilidad (y probabilidades) de eventos por lo que muchos de sus resultados son susceptibles de ser 
analizados por medio de la teoría de la información.

Por otro lado, de la interacción entre la teoría de la información y la mecánica cuántica surge un nuevo campo de la ciencia, la Teoría de la Información Cuántica, que estudia el procesamiento y los mecanismos de la información en sistemas que obedecen la mecánica cuántica [75]; la posibilidad del cómputo cuántico es uno de los resultados más conocidos.

\subsection{La entropía de Shannon}

Vamos a introducir la entropía de Shannon y a elaborar argumentos que sirvan para establecer su pertinencia, seguiremos el libro de Khinchin [76], cuyo trabajo fue fundamental para darle a la teoría de la información el estatus de teoría estadística.

Sea $A_{1}, A_{2}, \ldots, A_{n}$ un sistema completo de eventos, ${ }^{1}$ y sean $p_{1}, p_{2}, \ldots, p_{n}$ las probabilidades asociadas a cada uno de los eventos $\left(\sum_{i=1}^{n} p_{i}=1\right)$. Diremos que tenemos un esquema finito:

$$
A=\left(\begin{array}{cccc}
A_{1} & A_{2} & \ldots & A_{n} \\
p_{1} & p_{2} & \ldots & p_{n}
\end{array}\right) .
$$

Naturalmente, cada esquema finito describe un estado de incertidumbre. Representa un experimento en que sólo conocemos las probabilidades asociadas a cada uno de los eventos posibles. Diferentes esquemas finitos se asocian con diferentes estados de incertidumbre, por ejemplo si comparamos los dos esquemas:

$$
\left(\begin{array}{ll}
A_{1} & A_{2} \\
0.5 & 0.5
\end{array}\right), \quad\left(\begin{array}{cc}
A_{1} & A_{2} \\
0.99 & 0.01
\end{array}\right)
$$

resulta evidente que el primero representa un estado de incertidumbre mayor que el segundo. En el segundo esquema, el resultado del experimento será "casi seguramente" $A_{1}$, en tanto que seríamos cuidadosos con predecir el resultado del primer experimento si tuviésemos que apostar. El esquema,

\footnotetext{
${ }^{1}$ Es decir, un conjunto de eventos tales que uno y sólo uno de ellos ocurre a la vez.
} 


$$
\left(\begin{array}{ll}
A_{1} & A_{2} \\
0.4 & 0.6
\end{array}\right)
$$

representa un estado de incertidumbre intermedio entre los anteriores. Estamos interesados en establecer una medida cuantitativa de la incertidumbre en un esquema finito. Introduciremos la cantidad

$$
H\left(p_{1}, p_{2}, \ldots, p_{n}\right)=-\sum_{k=1}^{n} p_{k} \log p_{k}
$$

conocida como la entropía de Shannon y veremos que debido a sus propiedades puede servir como una medida de la incertidumbre en el esquema finito. Los logaritmos se toman en una base arbitaria, si la base del logaritmo es 2 , las unidades en que se mide son bits (contracción de "binary units") y si se utiliza el logaritmo natural, las unidades son nats ("natural units"), que son las que se utilizarán en este trabajo.

En este punto, hacemos notar que $\lim _{p_{k} \rightarrow 0} p_{k} \log p_{k}=0$.

$H\left(p_{1}, p_{2}, \ldots, p_{n}\right)=0$ si uno y sólo uno de los números $p_{1}, p_{2}, \ldots, p_{n}$ es uno y todos los demás son cero (debido a que $\sum_{i=1}^{n} p_{i}=1$ ). Este es el caso en que el resultado de un experimento puede predecirse con certidumbre, es decir que no hay incertidumbre. En todos los demás casos la entropía es positiva² ${ }^{2}$

Para un $n$ fijo el esquema de mayor incertidumbre es aquel en que todos los resultados tienen la misma probabilidad, es decir, $p_{k}=1 / n$ con $(k=1,2, \ldots, n)$, y de hecho la entropía asume su máximo valor en esta situación.

Ahora supongamos que tenemos dos esquemas finitos que son mutuamente independientes,

$$
A=\left(\begin{array}{cccc}
A_{1} & A_{2} & \ldots & A_{n} \\
p_{1} & p_{2} & \ldots & p_{n}
\end{array}\right) \quad B=\left(\begin{array}{cccc}
B_{1} & B_{2} & \ldots & B_{m} \\
q_{1} & q_{2} & \ldots & q_{m}
\end{array}\right)
$$

es decir, la probabilidad $\pi_{k l}$ de la ocurrencia conjunta de los eventos $A_{k}$ y $B_{l}$ es $p_{k} q_{l}$. Entonces el conjunto de eventos $A_{k} B_{l}(1 \leq k \leq n, 1 \leq l \leq m)$ con probabilidades $\pi_{k l}$

\footnotetext{
${ }^{2}$ Porque, $\log (x)<0$, si $x<1$.
} 
representa otro esquema finito que llamaremos el producto de los esquemas $A$ y $B$ y denotaremos por $A B$. Si $H(A), H(B)$ y $H(A B)$ son las entropías de los esquemas $A$, $B$ y $A B$, entonces,

$$
H(A B)=H(A)+H(B)
$$

Llevar a cabo el experimento del esquema $A B$ es equivalente a llevar a cabo los experimentos de los esquemas $A$ y $B$, y si son equivalentes, esperamos que la información que nos provee $A B$ sea equivalente a la suma de la información que nos proveen $A$ y $B$.

Si los esquemas son mutuamente dependientes denotamos por $q_{k l}$ la probabilidad (condicional) de que el evento $B_{l}$ del esquema $B$ ocurra dado que ha ocurrido el evento $A_{k}$ del esquema $A$, de manera que

$$
\pi_{k l}=p_{k} q_{k l}(1 \leq k \leq n, 1 \leq l \leq m)
$$

\section{Entonces}

$$
-H(A B)=\sum_{k} \sum_{l} p_{k} q_{k l}\left(\log p_{k}+\log q_{k l}\right)=\sum_{k} \sum_{l} q_{k l} p_{k} \log p_{k}+\sum_{k} p_{k} \sum_{l} q_{k l} \log q_{k l}
$$

$\sum_{l} q_{k l}=1$ para cualquier $k$, y la suma $-\sum_{l} q_{k l} \log q_{k l}$ puede considerarse como la entropía condicional $H_{k}(B)$ del esquema $B$ calculada sobre la suposición de que el evento $A_{k}$ del esquema $A$ ocurrió. Entonces,

$$
H(A B)=H(A)+\sum_{k} p_{k} H_{k}(B)
$$

La entropía condicional $H_{k}(B)$ es una variable aleatoria del esquema $A$ por lo que su valor queda totalmente determinado por el conocimiento del evento $A_{k}$ del esquema $A$ que efectivamente ocurre. Por lo tanto, el último término del lado derecho de la ecuación es el valor esperado de la cantidad $H(B)$ en el esquema $A$ que designaremos como $H_{A}(B)$. Así, en el caso más general,

$$
H(A B)=H(A)+H_{A}(B)
$$


En todos los casos

$$
H_{A}(B) \leq H(B)
$$

Lo que puede probarse por las propiedades que tiene cualquier función convexa. La interpretación de esta desigualdad es: La cantidad de información dada por la realización de un esquema $B$ sólo puede decrecer si un esquema $A$ se ha realizado antes ${ }^{3}$.

Agregaremos una nueva propiedad relacionada con incorporar un evento imposible (o cualquier número de ellos) a un esquema finito. Los esquemas,

$$
\left(\begin{array}{cccc}
A_{1} & A_{2} & \ldots & A_{n} \\
p_{1} & p_{2} & \ldots & p_{n}
\end{array}\right) \quad \mathrm{y} \quad\left(\begin{array}{ccccc}
A_{1} & A_{2} & \ldots & A_{n} & A_{n+1} \\
p_{1} & p_{2} & \ldots & p_{n} & 0
\end{array}\right)
$$

no son esencialmente diferentes, por lo que debe ocurrir,

$$
H\left(p_{1}, p_{2}, \ldots, p_{n}, 0\right)=H\left(p_{1}, p_{2}, \ldots, p_{n}\right) .
$$

En la siguiente sección listaremos las propiedades que hemos introducido para la entropía, estas propiedades son adecuadas para una medida de la información y por lo tanto de la incertidumbre de una distribución de probabilidades.

\subsection{Propiedades de la entropía de Shannon}

- P1. Si tenemos certeza de un resultado $\left(p_{i}=1, p_{j \neq i}=0\right)$, su realización no nos brinda información, y esta debe ser cero $H\left(p_{1}, p_{2}, \ldots, p_{n}\right)=0$.

- P2. Si dos eventos, $A$ y $B$, son independientes la información que aporta realizar el evento que se compone del producto de ambos, $A B$, debe ser igual a la que aporta la suma de los eventos individuales, $H(A B)=H(A)+H(B)$. Esta propiedad se conoce como extensividad de la entropía.

\footnotetext{
${ }^{3} \mathrm{Si}$ los esquemas son independientes, resulta evidente que el conocimiento de $A$ no afecta el conocimiento de $B$, por lo tanto este enunciado se funda en la posibilidad de correlaciones entre los eventos de los esquemas $A$ y $B$. Este punto lo retomaremos más adelante.
} 
- P3. $H\left(p_{1}, p_{2}, \ldots, p_{n}\right)$ tiene su mayor valor cuando todos los eventos son equiprobables $p_{k}=1 / n$ con $(k=1,2, \ldots, n)$, con $\sum_{k=1}^{n} p_{k}=1$.

- P4. En general, $H(A B)=H(A)+H_{A}(B)$.

- P5. $H\left(p_{1}, p_{2}, \ldots, p_{n}, 0\right)=H\left(p_{1}, p_{2}, \ldots, p_{n}\right)$.

- P6. $H\left(p_{1}, p_{2}, \ldots, p_{n}\right)$ es continua en todos sus argumentos.

Ahora mostraremos que la forma funcional de la entropía de Shannon es la única que cumple simultáneamente con estas propiedades.

\subsection{Teorema de unicidad}

Demostrar que la forma funcional de la entropía de Shannon es la única posible es probar el siguiente teorema de unicidad [76, 77].

\section{Teorema}

Sea $H\left(p_{1}, p_{2}, \ldots, p_{n}\right)^{4}$ una función definida para cualquier entero $n$ y para todos los valores $p_{1}, p_{2}, \ldots p_{n}$ tal que $p_{k} \geq 0(k=1,2, \ldots, n), \sum_{k=1}^{n} p_{k}=1 . p_{i}$ es la probabilidad de ocurrencia del evento $X_{i}$ perteneciente al esquema $X$. Si esta función tiene las propiedades P3, P4, P5 y P6, entonces:

$$
H\left(p_{1}, p_{2}, \ldots, p_{n}\right)=-\lambda \sum_{k=1}^{n} p_{k} \log p_{k}
$$

donde $\lambda$ es una constante positiva.

Prueba $[76,77]$.

Definamos primero $A(n)=H\left(\frac{1}{n}, \frac{1}{n}, \ldots, \frac{1}{n}\right)$. Dividiremos la prueba en tres partes:

(a)

Mostraremos primero que $A(n)=\lambda \log (n)$, así, estableciendo el teorema en el caso en que la variable $X$ está uniformemente distribuida. Por P5 y P3 tenemos,

$$
A(n)=H\left(\frac{1}{n}, \frac{1}{n}, \ldots, \frac{1}{n}, 0\right) \leq A(n+1)
$$

\footnotetext{
${ }^{4}$ Indistintamente, utilizaremos la notación $H(X) \equiv H\left(p_{1}, p_{2}, \ldots, p_{n}\right)$.
} 
Por lo tanto $A$ es una función no decreciente de $n$.

Ahora permitamos que $X_{1}, X_{2}, \ldots, X_{m}$ sea una variable aleatoria independiente e idénticamente distribuida, cada una con $r$ valores en su rango, de manera que $H\left(X_{j}\right)=$ $A(r), 1 \leq j \leq m$, entonces debido a $\mathrm{P} 4$, tenemos

$$
H\left(X_{1}, X_{2}\right)=H\left(X_{1}\right)+H\left(X_{2}\right)=2 A(r)
$$

por inducción tenemos,

$$
H\left(X_{1}, X_{2}, \ldots, X_{m}\right)=m A(r)
$$

Sin embargo, el vector $X=\left(X_{1}, X_{2}, \ldots, X_{m}\right)$ tiene $r^{m}$ resultados igualmente probables, por lo tanto,

$$
H\left(X_{1}, X_{2}, \ldots, X_{m}\right)=A\left(r^{m}\right)
$$

Entonces,

$$
A\left(r^{m}\right)=m A(r)
$$

Si utilizamos $n$ variables independientes idénticamente distribuidas cada una de rango $s$, debe cumplirse entonces,

$$
A\left(s^{n}\right)=n A(s)
$$

Ahora elijamos $r, s, n$ arbitrariamente y fijemos el valor de $m$ según,

$$
r^{m} \leq s^{n} \leq r^{m+1}
$$

Utilicemos el hecho de que $A$ es una función no decreciente para obtener,

$$
A\left(r^{m}\right) \leq A\left(s^{n}\right) \leq A\left(r^{m+1}\right)
$$

por lo tanto,

$$
m A(r) \leq n A(s) \leq(m+1) A(r)
$$

esto es

$$
\frac{m}{n} \leq \frac{A(s)}{A(r)} \leq \frac{m}{n}+\frac{1}{n}
$$


y por lo tanto,

$$
\left|\frac{A(s)}{A(r)}-\frac{m}{n}\right| \leq \frac{1}{n}
$$

Si tomamos el logaritmo de las cantidades en la desgiualdad (4.9) tenemos,

$$
m \log (r) \leq n \log (s) \leq(m+1) \log (r)
$$

por un argumento similar al de arriba, tenemos,

$$
\left|\frac{\log (s)}{\log (r)}-\frac{m}{n}\right| \leq \frac{1}{n}
$$

Ahora, utilizando la desigualdad del triángulo de dos números reales $a$ y $b,|a+b| \leq$ $|a|+|b|$, obtenemos,

$$
\begin{aligned}
\left|\frac{A(s)}{A(r)}-\frac{\log (s)}{\log (r)}\right| & =\left|\left(\frac{A(s)}{A(r)}-\frac{m}{n}\right)+\left(\frac{m}{n}-\frac{\log (s)}{\log (r)}\right)\right| \\
& \leq\left|\frac{A(s)}{A(r)}-\frac{m}{n}\right|+\left|\frac{m}{n}-\frac{\log (s)}{\log (r)}\right| \\
& \leq \frac{2}{n} \quad \text { debido a (4.12) y (4.14) }
\end{aligned}
$$

y como podemos hacer $n$ arbitrariamente grande,

$$
\frac{A(s)}{A(r)}=\frac{\log (s)}{\log (r)}
$$

de donde podemos deducir que $A(s)=\lambda \log (s)$. El hecho de que $A(s)$ es una función no decreciente, lo cual se ha mostrado antes, conduce a que $\lambda \geq 0$.

Con este resultado completamos la parte (a) de la prueba.

(b)

Probaremos ahora el teorema en el caso en que cada $p_{j}$ es un número racional, entonces puede escribirse,

$$
p_{j}=\frac{m_{j}}{m}, \quad \text { donde } \quad \sum_{j=1}^{n} m_{j}=m
$$

Ahora introducimos una nueva variable aleatoria $Y$ que tiene $m$ valores y que dividiremos en $n$ grupos como sigue,

$$
y_{11}, y_{12}, \ldots y_{1 m_{1}}, y_{21}, y_{22}, \ldots y_{2 m_{2}}, \ldots, y_{n 1}, y_{n 2}, \ldots y_{n m_{n}}
$$


La razón de que agrupemos de esta manera es que queremos que $Y$ dependa de $X$, lo que se logra definiendo las probabilidades condicionales (donde condicionamos sobre el evento $\left.X=x_{r}\right)$

$$
\begin{gathered}
P_{r}\left(Y=y_{r k}\right)=\frac{1}{m_{r}} \quad \text { para } \quad 1 \leq k \leq m_{r} \\
P_{r}\left(Y=y_{r k}\right)=0 \quad \text { para } \quad 1 \leq k \leq m_{s}, s \neq r
\end{gathered}
$$

para $1 \leq r \leq n$

Obtenemos así $H_{r}(Y)=\lambda \log \left(m_{r}\right)$ debido a la parte (a) de la prueba y por lo tanto,

$$
H_{X}(Y)=\sum_{r=1}^{n} p_{r} H_{r}(Y)=\lambda \sum_{r=1}^{n} \frac{m_{r}}{m} \log \left(m_{r}\right) .
$$

Por otro lado tenemos las probabilidades conjuntas

$$
\begin{aligned}
P\left(\left(X=x_{r}\right) \cap\left(Y=y_{s k}\right)\right) & =p_{r} P_{r}\left(Y=y_{s k}\right)=0 \quad \text { cuando } \quad s \neq r \\
& =\frac{m_{r}}{m} \times \frac{1}{m_{r}}=\frac{1}{m} \quad \text { para cada } \quad 1 \leq k \leq m_{r}
\end{aligned}
$$

Entonces, otra vez debido a (a) y a P5 podemos deducir que

$$
H(X, Y)=\lambda \log (m)
$$

Ahora, debido a P4, encontramos,

$$
\begin{aligned}
H(X) & =H(X, Y)-H_{X}(Y) \\
& =\lambda \log (m)-\lambda \sum_{r=1}^{n} \frac{m_{r}}{m} \log \left(m_{r}\right) \\
& =-\lambda \sum_{r=1}^{n} \frac{m_{r}}{m} \log \left(\frac{m_{r}}{m}\right), \quad \text { como queríamos }
\end{aligned}
$$

donde hemos usado el hecho que $\sum_{r=1}^{n} \frac{m_{r}}{m}=1$.

Con esto completamos la prueba de (b).

(c)

Ahora permitamos que las probabilidades sean números reales arbitrarios, de manera que cada $p_{j}$ puede aproximarse por una secuencia de racionales $p_{j}^{(N)}$, donde cada 
$p_{j}^{(N)}$ puede escribirse en la forma dada en $(\mathbf{b})$. Sea entonces $U^{(N)}(X)$ la correspondiente secuencia de entropías y definamos

$$
U(X)=-\sum_{j=1}^{n} p_{j} \log \left(p_{j}\right)
$$

entonces tenemos que

$$
U(X)=\lim _{N \rightarrow \infty} U^{(N)}(X) .
$$

Sin embargo, por la condición de continuidad, P6, y el resultado en (b) también tenemos

$$
H(X)=\lim _{N \rightarrow \infty} U^{(N)}(X)
$$

por lo tanto, debido a la unicidad del límite, debemos tener $H(X)=U(X)$, con lo cual completamos la prueba del teorema.

\subsection{Entropía discreta y entropía diferencial}

Hemos incluido la prueba anterior debido a la relevancia que tiene mostrar la unicidad de la entropía de Shannon en el caso discreto. La entropía de Shannon puede generalizarse al caso continuo para ello se procede por analogía con el caso discreto.

Esta generalización tiene algunas particularidades, una de las más importantes es que la densidad de probabilidad, en contraste con las probabilidades del caso discreto, no está acotada ahora en el intervalo $(0,1)$ y la entropía de Shannon puede ahora adoptar valores negativos.

En el mismo sentido, en el caso continuo la propiedad P5 que hemos usado en la prueba de la sección anterior no tiene un análogo claro.

Por otro lado, respecto a la propiedad P3: la entropía en el caso continuo también tiene el valor máximo cuando la distribución es constante (eventos equiprobables). Para que esta densidad de probabilidad sea cuadráticamente integrable, integre a la unidad $^{5}$, la función debe acotarse a un intervalo, por ejemplo $(a, b)$, y la función debe

\footnotetext{
${ }^{5}$ Como solicitaremos de toda función que pueda llamarse una densidad de probabilidad.
} 
ser igual a $1 /(a-b)$. Sólo de esta forma puede cumplirse que,

$$
\int_{a}^{b} f(x) d x=\int_{a}^{b} \frac{1}{a-b} d x=1
$$

Si pensamos en sistemas que cumplen restricciones físicas, por ejemplo los sistemas cuánticos, hay dos alternativas: 1) una función que es constante no es cuadráticamente integrable si se define en el intervalo $(-\infty, \infty)$ ó $(0, \infty)$; ó 2$)$ una función que cumple con la Ec. (4.27) no cumple con las condiciones a la frontera de Dirichlet para estos sistemas confinados ${ }^{6}$. Entonces la propiedad P3 no siempre tiene un análogo en el caso continuo.

Debemos señalar que la entropía de Shannon en el caso continuo no es invariante al cambio de coordenadas.

En sistemas cuánticos, el uso de la entropía discreta es habitual, se utilizan los eigenvalores de la matriz de la densidad y se le conoce como la entropía de von Neumann o la entropía de Jaynes. En esta tesis estamos interesados en tratar la incertidumbre asociada a la propia densidad en los espacios de posición, momento y en un espacio fase cuántico, es decir, tratamos con variables continuas, por ello utilizaremos la formulación continua de la entropía de Shannon. Enseguida introduciremos el resto de los conceptos entrópicos que utilizaremos en su versión continua.

\subsection{La entropía diferencial de Shannon}

Si tenemos una distribución de probabilidad de la variable aleatoria $x, f(x)$, que cumple con $\int d x f(x)=1$. La entropía diferencial de Shannon (o simplemente entropía de Shannon) se define [74],

$$
s=-\int d x f(x) \ln f(x)
$$

\footnotetext{
${ }^{6}$ Sin embargo, es posible encontrar problemas que tienen condiciones a la frontera periódicas, por ejemplo el estado basal de una partícula en una circunferencia, también el sistema de dos partículas no interactuantes en una circunferencia. Ahí no hay problema y se cumple la normalización y las condiciones a la frontera.
} 
Es posible definir la entropía de Shannon $\left(s_{N}\right)$ si la distribución está normalizada a un número, por ejemplo $N$, cuya relación con la Ec. (4.28) es,

$$
s=\frac{s_{N}}{N}+\ln (N) \text {. }
$$

A la Ec. (4.28) puede llamársele la entropía del factor de forma (shape function), $\rho(x) / N$. Para ver una discusión del factor de forma citamos [78].

Es posible definir una entropía relativa que es una "distancia" estadística de una distribución de probabilidad respecto de una referencia, $q(x)$ [79],

$$
s(f, q)=\int d x f(x) \ln \frac{f(x)}{q(x)} .
$$

Usando la propiedad de convexidad, se demuestra que $s(f, q) \geq 0$, con la igualdad sí y sólo sí $f(x)=q(x)[80]$.

Para casos bidimensionales se puede definir una entropía conjunta. Dada una distribución de probabilidad conjunta que cumpla con todas las propiedades exigidas por la teoría de la probabilidad, $f(x, p)$, definimos la entropía de Shannon de dos variables,

$$
s_{x_{1}, x_{2}}=-\iint d x_{1} d x_{2} f\left(x_{1}, x_{2}\right) \ln f\left(x_{1}, x_{2}\right) .
$$

Si definimos,

$$
s_{x_{1}}=-\int d x_{1} f_{1}\left(x_{1}\right) \ln f_{1}\left(x_{1}\right)
$$

$\mathrm{y}$

$$
s_{x_{2}}=-\int d x_{2} f_{2}\left(x_{2}\right) \ln f_{2}\left(x_{2}\right)
$$

con,

$$
f_{1}\left(x_{1}\right)=\int d x_{2} f\left(x_{1}, x_{2}\right)
$$

$\mathrm{y}$

$$
f_{2}\left(x_{2}\right)=\int d x_{1} f\left(x_{1}, x_{2}\right)
$$

puede verificarse que la entropía conjunta cumple con la propiedad de subaditividad, como se señaló antes para el caso discreto,

$$
s_{x_{1}, x_{2}} \leq s_{x_{1}}+s_{x_{2}}
$$


y la igualdad se satisface sólo si los dos eventos son independientes, es decir $f\left(x_{1}, x_{2}\right)=$ $f_{1}\left(x_{1}\right) f_{2}\left(x_{2}\right)$.

Se puede definir también una entropía relativa para distribuciones de más de una variable introduciendo una distribución de referencia, $q\left(x_{1}, x_{2}\right)$, como sigue,

$$
s(f, q)=\iint d x_{1} d x_{2} f\left(x_{1}, x_{2}\right) \ln \frac{f\left(x_{1}, x_{2}\right)}{q\left(x_{1}, x_{2}\right)} .
$$

que tiene un caso particular muy importante cuando la referencia es el producto de las marginales de la distribución porque es una medida de la correlación entre dos variables. El nombre que recibe esta entropía relativa es Información mutua,

$$
I_{x_{1}, x_{2}}=\iint d x_{1} d x_{2} f\left(x_{1}, x_{2}\right) \ln \frac{f\left(x_{1}, x_{2}\right)}{f_{1}\left(x_{1}\right) f_{2}\left(x_{2}\right)}=s_{x_{1}}+s_{x_{2}}-s_{x_{1}, x_{2}},
$$

y mide qué tan separable es la distribución de dos variables en sus marginales. Se desprende de la propiedad de subaditividad que la información mutua siempre es no negativa y sólo es cero cuando los eventos son independientes, $f\left(x_{1}, x_{2}\right)=f_{1}\left(x_{1}\right) f_{2}\left(x_{2}\right)$.

\subsection{Problemas observados en las entropías diferen- ciales}

Es pertinente señalar algunos problemas que hemos observado en el cálculo de las entropías. El primero es la negatividad de una entropía relativa.

En la Ec. (4.30) se muestra que, $s(f, q) \geq 0$, y el único caso donde $s(f, q)=0$, es $f(x)=q(x)$. A pesar de lo anterior hemos obervado, en nuestros resultados y en la literatura, que en el caso en que $q(x)=c$ y $c$ es una constante, puede ocurrir que $s(f, q)<0$.

Un caso importante de este problema está relacionado con las unidades de la entropía de Shannon.

Cuando calculamos la entropía de Shannon el argumento del logartimo debe carecer de unidades (en un caso físico); usualmente para conseguirlo la convención es que se 
divide el argumento del logaritmo por la unidad con dimensiones adecuadas ${ }^{7}$. En este caso la entropía de Shannon puede ser negativa, según lo anotado en el párrafo anterior.

Otra forma de resolver el problema de las dimensiones del argumento del logaritmo es utilizar una densidad de probabilidad adimensional, en el caso del espacio de posición lo logramos dividiendo adecuadamente por alguna "longitud característica" en el problema. Dicho sea de paso, en la entropía relativa, Ecs. (4.30) y (4.37), el argumento del logaritmo es adimensional porque se cancelan las de ambas distribuciones.

El segundo problema que señalaremos se refiere a la necesidad de que ambas distribuciones en la entropía relativa tengan nodos en los mismos puntos.

Para la entropía relativa, Ecs. (4.30) y (4.37) es necesario calcular, respectivamente,

$$
\int d x f(x) \ln g(x) \quad \int d x_{1} d x_{2} f\left(x_{1}, x_{2}\right) \ln g\left(x_{1}, x_{2}\right)
$$

si $f$ y $g$ tienen nodos en diferentes lugares estos términos divergen. Esto complica el cálculo, por ejemplo, de una distancia estadística entre los estados del oscilador armónico si la que se utiliza como referencia es una distribución diferente al estado basal. También se encuentra el problema si se calcula la distancia estadística entre una referencia antisimétrica y una función simétrica.

Estos problemas son propios de la versión continua de la entropía de Shannon.

\subsection{Medidas de incertidumbre cuántica}

Enfrentados al problema de medir incertidumbre cuántica es posible optar por la desviación estándar o por la entropía de Shannon. Primero liguemos el concepto de incertidumbre con el de localización.

Si tenemos dos distribuciones como las mostradas en la Fig. (4.1), sea que provengan de un experimento o de la resolución de una ecuación diferencial, visualmente podemos concluir que la gráfica de abajo tendrá una mayor desviación estándar y por tanto

\footnotetext{
${ }^{7}$ Es una entropía relativa donde la referencia es una constante, aprovechando que $\ln (1)=0$. Puede verse una discusión en [81].
} 
tendrá una mayor incertidumbre asociada, que la de arriba. También visualmente podemos establecer que la distribución de arriba se encuentra más localizada ${ }^{8}$.
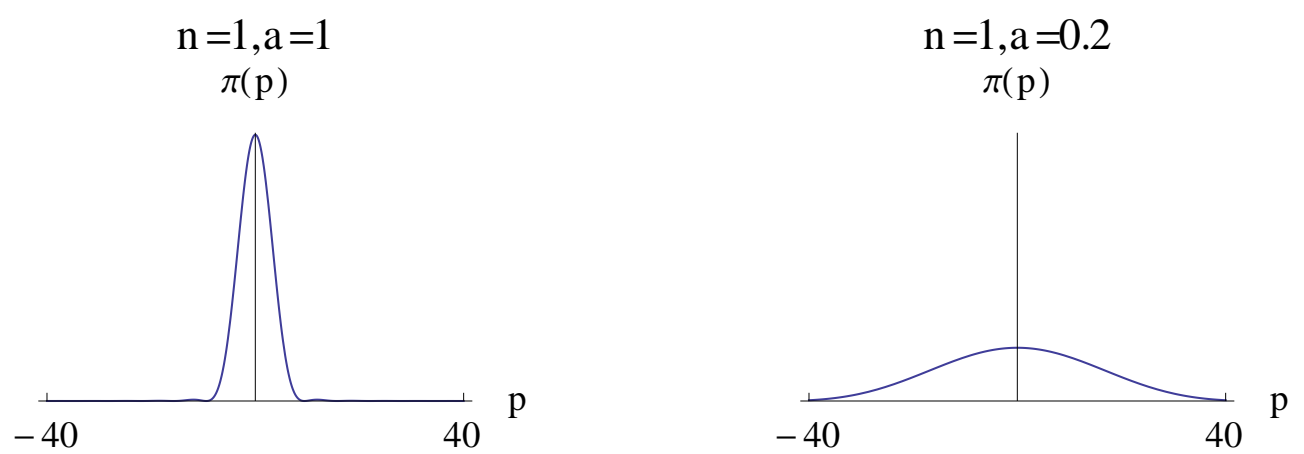

Figura 4.1: Densidad de probabilidad del estado basal de la partícula en la caja en el espacio de momento, para diferentes valores de tamaño de caja.

La desviación estándar es una medida de la incertidumbre que es exacta si tratamos con distribuciones gaussianas, está formulada dentro de ese tipo de distribuciones. La función general de una gaussiana es,

$$
f(x)=\frac{1}{\Delta x \sqrt{2 \pi}} e^{-\frac{1}{2}\left(\frac{x-\langle x\rangle}{\Delta x}\right)^{2}}
$$

La entropía de Shannon es,

$$
s_{x}=\int d x f(x) \ln f(x)=\ln (\Delta x \sqrt{2 \pi e})
$$

Entonces en las gaussianas, donde es exacta la desviación estándar, la entropía de Shannon es consistente con ésta. $s_{x}$ crece si $\Delta x$ crece, por tanto recupera su comportamiento.

La pregunta debemos dirigirla entonces hacia las distribuciones que se apartan del comportamiento gaussiano, ¿qué medida de incertidumbre será más adecuada?

\footnotetext{
${ }^{8} \mathrm{Si}$ se trata de datos de un experimento, éstos están localizados alrededor del valor medio. Si se trata de la densidad de probabilidad de encontrar partículas en algún sitio, éstas están localizadas en la región del espacio alrededor del valor esperado de la distribución.
} 
Hemos mencionado que la desviación estándar depende en gran medida del valor medio de la distribución. En contraste la entropía de Shannon es una cantidad global que no depende de un punto específico de la distribución, además, se ha mostrado su dependencia en el tamaño relativo de los picos de la distribución y con ello su capacidad de reflejar esta característica estructural [82].

Pueden encontrarse problemas apartados del comportamiento gaussiano en los que el valor esperado de la variable es cero, por ejemplo $\langle p\rangle=0$, pero la distribución no está concentrada alrededor de ese punto. Nos serviremos de un modelo canónico de la mecánica cuántica para ejemplificar este comportamiento [83].

La Fig. (4.2) muestra la densidad de momentos de diferentes estados de la partícula en la caja, $\pi(p)$. El hecho de que $\langle p\rangle=0$ indica que, por su carácter vectorial, el momento tiene la misma probabilidad de ser positivo o negativo y no demuestra que la distribución está concentrada alrededor de $p=0$. Este es un caso muy común, por ejemplo en los sistemas con un potencial central.

Conforme aumenta el número cuántico, $n$, se alejan los picos del origen lo que muestra un aumento en el valor absoluto del momento promedio de las partículas ${ }^{9}$. Conforme $n$ va creciendo puede verificarse visualmente que la estructura de la distribución cambia cada vez menos.

La Fig. (4.3) muestra la desviación estándar y la entropía de Shannon de la distribución de la Fig (4.2). El comportamiento de la desviación estándar $\Delta p$ se debe a que los picos se alejan del origen y crece linealmente con $n$, esto refleja su dependencia en un punto específico de la distribución, $\langle p\rangle=0$.

En contraste, la entropía de Shannon, $s_{p}$, se comporta asintóticamente con $n$, y refleja mejor el hecho de que la estructura de la distribución cambia poco. En este caso, concluimos que $s_{p}$ es una medida de la incertidumbre más conveniente que $\Delta p$, dado el comportamiento no gaussiano de la densidad.

\footnotetext{
${ }^{9}$ En este modelo sólo hay energía cinética, $E_{c}$, entonces si aumentamos la energía con $n$, estamos aumentando $E_{c}$ y tiene que crecer el valor absoluto del momento promedio. Existe una relación con $\Delta p$ porque ambos dependen en el segundo momento de la distribución, $E_{c}=\left\langle p^{2}\right\rangle / 2 m$, con $m$ la masa.
} 

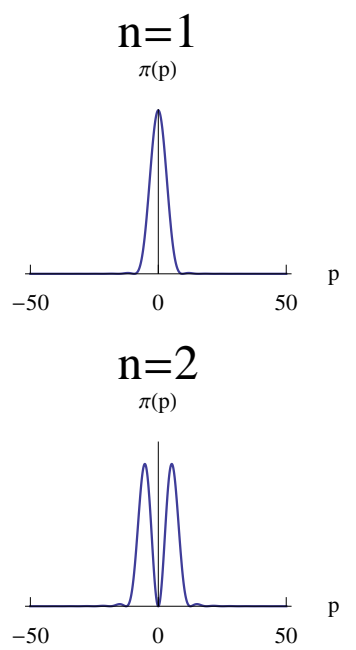

$$
\mathrm{n}=5
$$
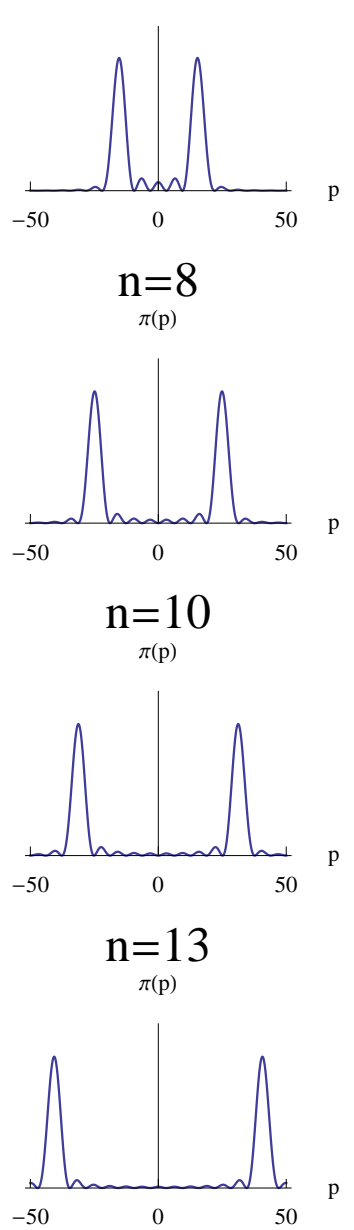

Figura 4.2: Densidad de momentos, $\pi(p)$, para diferentes estados de la partícula en la caja. Sólo hay variación en la escala para $n=1$. 
La conveniencia de una u otra medida es un problema particular que debe resolverse en cada caso de análisis, no obstante, estos ejemplos son evidencia de que una cantidad como la entropía podría medir la incertidumbre mejor que el segundo momento de la distribución. Otra perspectiva de esta observación refiere a las fluctuaciones que usualmente se miden por medio del segundo momento de la distribución; si hiciéramos este análisis con una partícula en una caja no obtendríamos conclusiones adecuadas.
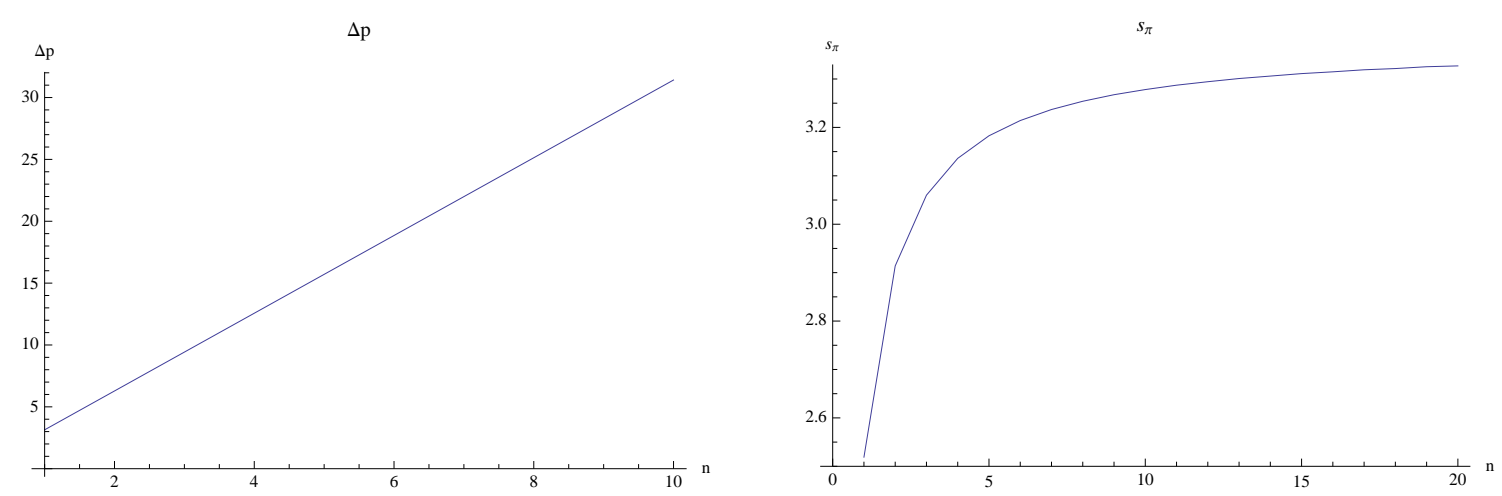

Figura 4.3: Desviación estándar (izquierda) y entropía de Shannon (derecha) en el espacio de momento, como función del estado cuántico, de una partícula en una caja.

Existen trabajos que muestran una relación de la entropía de Shannon con cantidades experimentales en la Aproximación de Plasma Local (LPA) [84, 85]. La posibilidad de medir experimentalmente la entropía de Shannon es un campo que se investiga actualmente [86]. También existe evidencia numérica de que cantidades entrópicas están relacionadas con propiedades como la energía de correlación [87, 88, 89, 90].

La cuestión es si la entropía de Shannon es una propiedad del sistema y puede medirse en un experimento. En cualquier caso sus aplicaciones como parte de la teoría estadística son independientes de ello.

Entre otras posibles aplicaciones, los vínculos entre la teoría de funcionales de la densidad y la teoría de la información se han estrechado gradualmente y la discusión sobre su pertinencia es una actual, para ver una de las polémicas referimos a [91, 92]. Por otro lado, la entropía de Shannon en el espacio de posición $\left(s_{\rho}\right)$ ha sido relacionada 
con la energía de correlación en un gas de electrones [93].

La entropía de Shannon es un funcional de la densidad y puede considerarse una función de respuesta cuyo análisis brinda información sobre el comportamiento de una cantidad que definitivamente es física, la densidad de carga o la densidad de momento.

En sistemas químicos, se han estudiado las entropías de Shannon mono-electrónicas [94, 95, 96, 97, 98, 99, 100, 101, 102, 103, 104], y también las entropías de pares $[30,105,106,107]$.

\subsection{Medidas de la correlación estadística en sis- temas cuánticos}

Enfrentados ahora al problema de medir correlaciones estadísticas, tenemos como opciones el coeficiente de correlación (que es la versión normalizada de la covarianza) y la información mutua.

Un problema típico de correlación donde las distribuciones son gaussianas, es la distribución normal bivariada, que tiene la forma,

$$
\begin{aligned}
f\left(x_{1}, x_{2}\right) & =\frac{1}{2 \pi \Delta x_{1} \Delta x_{2}\left(1-\tau^{2}\right)^{1 / 2}} \\
& \times \exp \left[-\frac{1}{2\left(1-\tau^{2}\right)}\left(\frac{x_{1}^{2}}{\left(\Delta x_{1}\right)^{2}}-2 \tau \frac{x_{1} x_{2}}{\Delta x_{1} \Delta x_{2}}+\frac{x_{2}^{2}}{\left(\Delta x_{2}\right)^{2}}\right)\right],
\end{aligned}
$$

donde $\Delta x_{1}$ y $\Delta x_{2}$ son las desviaciones estándar de ambas variables, y $\tau$ es el coeficiente de correlación, definido por,

$$
\tau=\frac{\left\langle x_{1} x_{2}\right\rangle-\left\langle x_{1}\right\rangle\left\langle x_{2}\right\rangle}{\sqrt{\left\langle x_{1}^{2}\right\rangle-\left\langle x_{1}\right\rangle^{2}} \sqrt{\left\langle x_{2}^{2}\right\rangle-\left\langle x_{2}\right\rangle^{2}}} .
$$

con

$$
\begin{gathered}
-1 \leq \tau \leq 1 \\
\left\langle x_{1} x_{2}\right\rangle=\int d x_{1} d x_{2} x_{1} x_{2} f\left(x_{1}, x_{2}\right), \quad\left\langle x_{1}\right\rangle=\int d x_{1} d x_{2} x_{1} f\left(x_{1}, x_{2}\right)
\end{gathered}
$$




$$
\begin{gathered}
\left\langle x_{2}\right\rangle=\int d x_{1} d x_{2} x_{2} f\left(x_{1}, x_{2}\right), \quad\left\langle x_{1}^{2}\right\rangle=\int d x_{1} d x_{2} x_{1}^{2} f\left(x_{1}, x_{2}\right) \\
\left\langle x_{2}^{2}\right\rangle=\int d x_{1} d x_{2} x_{2}^{2} f\left(x_{1}, x_{2}\right)
\end{gathered}
$$

La información mutua, Ec. (4.38), para esta distribución es [108],

$$
I_{x_{1}, x_{2}}=-\frac{1}{2} \ln \left(1-\tau^{2}\right)
$$

puede notarse que no depende de las desviaciones estándar, sólo del coeficiente de correlación; crece cuando $\tau$ aumenta, y varía en el intervalo $(0, \infty)$. Si el sistema está débilmente correlacionado, $|\tau| \ll 1$, entonces $I_{x_{1}, x_{2}} \approx \tau^{2} / 2$.

Es sencillo encontrar problemas donde el coeficiente de correlación es cero y la distribución no es separable. Debido a simetrías en el problema puede ocurrir que $\left\langle x_{1} x_{2}\right\rangle=0$, y si además $\left\langle x_{1}\right\rangle=0$ el coeficiente es cero sin necesidad de que la función sea separable.

Por otro lado, el coeficiente de correlación es sensible a la correlación lineal entre variables en tanto que la información mutua detecta, además de esa, correlaciones no lineales.

La información mutua es una medida más general de correlación estadística que el coeficiente de correlación. Es posible utilizar la curtosis y otros momentos más altos de la distribución para estudiar la correlación que no detecta el segundo momento. La comparación de la información mutua con la curtosis u otros momentos de la distribución es un tema que aún está abierto.

Por otra parte, en sistemas químicos se ha estudiado el coeficiente de correlación [109] y también se ha introducido la información mutua [110, 111]

\subsection{Relaciones entrópicas de incertidumbre}

Como hemos visto, mediante el cálculo de las entropías de Shannon podemos medir la incertidumbre en el espacio de posición y en el espacio de momento, la suma de estas entropías debe cumplir una cota para ser consistente con el principio de incertidumbre. 
Bialynicki-Birula y Mycielski [112] establecieron una relación de incertidumbre para las entropías de Shannon en los espacios de posición y momento,

$$
s_{\rho}+s_{\pi} \geq 1+\ln \pi
$$

donde,

$$
s_{\rho}=-\int d x \rho(x) \ln \rho(x)
$$

y

$$
s_{\pi}=-\int d p \pi(p) \ln \pi(p) .
$$

La cota proviene de la transformada de Dirac-Fourier que relaciona las funciones de onda. La suma entrópica puede expresarse como la entropía de una distribución separable en espacio fase [108],

$$
s_{\rho}+s_{\pi}=-\int d x d p \rho(x) \pi(p) \ln \rho(x) \pi(p) .
$$

De esta manera puede relacionarse la localización en un espacio fase separable con las relaciones de incertidumbre. Una generalización importante es la definición de una entropía que utilice distribuciones en espacio fase no separables, como la función de Wigner.

La suma entrópica es el análogo a la relación de incertidumbre que escribimos en términos de las desviaciones estándar, en las Ecs. (2.7), (2.9) y (2.10). Hacemos notar que son ecuaciones para una partícula.

Podemos definir también entropías de pares, que son medidas de la incertidumbre en las distribuciones de dos partículas por lo que existe entre ellas una relación de incertidumbre. Esta es una diferencia notable con la desviación estándar, porque no existe una manera sencilla de definir la incertidumbre de dos variables ${ }^{10}$.

Las entropías de Shannon de pares se definen en ambos espacios,

\footnotetext{
${ }^{10}$ Debe usarse en ese caso la matriz de covarianzas.
} 


$$
\begin{aligned}
& s_{\Gamma}=-\int d x_{1} d x_{2} \Gamma\left(x_{1}, x_{2}\right) \ln \Gamma\left(x_{1}, x_{2}\right) \\
& s_{\Pi}=-\int d p_{1} d p_{2} \Pi\left(p_{1}, p_{2}\right) \ln \Pi\left(p_{1}, p_{2}\right)
\end{aligned}
$$

La relación de incertidumbre de dos partículas debe cumplir la cota [106],

$$
s_{\Gamma}+s_{\Pi} \geq 2(1+\ln \pi)
$$

y puede verse como una medida de la localización en un espacio fase separable de dos partículas,

$$
s_{\Gamma}+s_{\Pi}=\int d x_{1} d x_{2} d p_{1} d p_{2} \Gamma\left(x_{1}, x_{2}\right) \Pi\left(p_{1}, p_{2}\right) \ln \left[\Gamma\left(x_{1}, x_{2}\right) \Pi\left(p_{1}, p_{2}\right)\right] .
$$

También en este caso es importante generalizar esta expresión utilizando una densidad no separable, como la función de Wigner.

\subsection{Información mutua entre posición y momento, $I_{x, p}$}

Las incertidumbres de la posición y del momento están correlacionadas en el principio de incertidumbre por lo que pueden variar siempre y cuando cumplan la cota. Queremos estudiar la correlación entre las propias variables $x$ y $p$.

Una primera opción de estudio es el coeficiente de correlación, pero éste es cero para funciones de onda reales [25], por lo que la correlación posición-momento está relacionada con la fase de la función. Por otro lado, el hecho de que el coeficiente es cero no indica que no existe correlación estadística entre la posición y el momento, sino que no puede detectarla. La información mutua es una medida de correlación más general y es una segunda opción.

En la Ec. (4.47) se observa que las variables no están correlacionadas en la distribución separable; para estudiar esta correlación debemos estudiar una distribución 
no separable cuyas marginales sean la densidad de posición y de momento. Como argumentamos anteriormente la función de Wigner cumple con estos requisitos.

Utilizando la función de Wigner y la información mutua podemos medir la correlación entre posición y momento mediante,

$$
I=\int d x d p W(x, p) \ln \frac{W(x, p)}{\rho(x) \pi(p)}=s_{\rho}+s_{\pi}-s_{w}
$$

donde $s_{w}$ es la entropía de Shannon de la función de Wigner, que definiremos enseguida.

Es importante notar en esta expresión que la correlación está definida como la diferencia entre la localización de la función de Wigner y la suma entrópica, que forma la base del principio de incertidumbre. Localización y correlación son entonces dos conceptos que no son lejanos.

\subsection{Entropía de la función de Wigner, $s_{w}$}

\subsubsection{Definición}

Se ha sugerido que la correlación entre posición y momento podría detectarse estudiando la estructura de la función de Wigner. La correlación posición-momento se manifiesta en los picos de la distribución [113]. La entropía de Shannon de la función de Wigner es una medida cuantitativa de su estructura (localización), por lo tanto debe contener la información de tales picos.

La información mutua entre posición y momento se basa en el compromiso entre la localización de la función de Wigner y la distribución separable del producto de las densidades.

Wehrl introdujo el concepto de entropía de una distribución en espacio fase cuántico [114] y la definió en términos de la función de Husimi [64, 62].

En el espíritu de la entropía de Wehrl definimos la entropía de Shannon de la función de Wigner. El problema de calcular esta entropía se ha discutido en la literatura y existen varias objeciones cuyo argumento es la existencia de las regiones negativas en 
la distribución [61, 63].

Sin embargo, es posible definir la entropía de Shannon de la función de Wigner, $s_{w}$, que aparece en la Ec. (4.52) como [108, 106],

$$
s_{w}=-\int d x d p W(x, p) \ln W(x, p) .
$$

La regiones negativas de la función de Wigner y la presencia del logaritmo provocan la aparición de un componente imaginario de la entropía, puesto que el cálculo del logaritmo de un número negativo sólo requiere especificar en que rama del logaritmo estamos trabajando [115].

Para calcular $s_{w}$ separamos las regiones donde la función de Wigner es positiva de las regiones donde es negativa. Si se procede numéricamente esta separación es aún más natural. Así, la normalización se calcula,

$$
N_{w}=\int_{+} d x d p W^{+}(x, p)+\int_{-} d x d p W^{-}(x, p)
$$

donde $\int_{+} \mathrm{y} \int_{-}$denotan que el espacio de integración es aquel donde la función de Wigner es positiva $(+)$ y negativa $(-)$, con la misma simbología para $W^{+}(x, p)$ y $W^{-}(x, p)$.

Para calcular la entropía escribimos,

$$
\begin{aligned}
s_{w}= & -\int_{+} d x d p W^{+}(x, p) \ln W^{+}(x, p) \\
& -\int_{-} d x d p W^{-}(x, p) \ln W^{-}(x, p)
\end{aligned}
$$

y el segundo término puede factorizarse,

$$
\begin{aligned}
-\int_{-} d x d p W^{-}(x, p) \ln W^{-}(x, p) & =-\int_{-} d x d p W^{-}(x, p) \ln \left[(-1)\left|W^{-}(x, p)\right|\right] \\
& =-\int_{-} d x d p W^{-}(x, p) \ln \left|W^{-}(x, p)\right| \\
& -\int_{-} d x d p W^{-}(x, p) \ln (-1) \\
& =-\int_{-} d x d p W^{-}(x, p) \ln \left|W^{-}(x, p)\right| \\
& -i \pi \int_{-} d x d p W^{-}(x, p)
\end{aligned}
$$


donde las barras "| |" denotan el valor absoluto, además hemos utilizado la convención de trabajar en la rama principal del logaritmo. Podemos separar las partes real e imaginaria de $s_{w}$,

$$
\begin{gathered}
\operatorname{Re}\left[s_{w}\right]=-\int_{+} d x d p W^{+}(x, p) \ln W^{+}(x, p) \\
+\int_{-} d x d p\left|W^{-}(x, p)\right| \ln \left|W^{-}(x, p)\right| \\
\operatorname{Im}\left[s_{w}\right]=-i \pi \int_{-} d x d p W^{-}(x, p) .
\end{gathered}
$$

Debido a que la función de Wigner está acotada ${ }^{11}$ según la Ec. (3.32), el signo del primer término de la Ec. (4.57) siempre es positivo y por lo tanto contibuye a deslocalizar la distribución (aumenta el valor de la entropía), en tanto que el segundo término siempre es negativo y contribuye a localizarla. La interpretación de esto es que las regiones positivas de la función de Wigner contribuyen deslocalizando el sistema en el espacio fase en tanto que las regiones negativas lo localizan.

La parte imaginaria de la entropía, Ec. (4.58), es proporcional al volumen de las regiones negativas de la función de Wigner. Si sabemos el valor del componente imaginario de la entropía y dividimos por $\pi$ tendremos el volumen. Se ha señalado la importancia y la necesidad de algún esquema para estimar ese volumen [116]. Este hecho es interesante porque las regiones negativas de la función de Wigner se han asociado con fenómenos cuánticos como el entrelazado $[116,117]$ y el volumen de estas regiones se ha utilizado como un criterio para estimar cuál sistema está más entrelazado [116].

\subsubsection{Interpretación de $s_{w}$}

En esta parte plantearemos tres preguntas necesarias para la discusión que se desarrollará a lo largo de esta tesis.

\footnotetext{
${ }^{11}$ En unidades atómicas $(\hbar=1)$ es siempre menor que 1 , y $\ln x$, si $x<1$, es un número negativo.
} 


\section{¿Por qué trabajar con la función de Wigner y no con alguna otra función de distribución en el espacio fase?}

La función de Wigner tiene las marginales correctas y el producto de esas densidades es la distribución en espacio fase separable a la cual está asociada la relación entrópica de incertidumbre. Además, la función de Wigner puede reconstruirse a partir de datos experimentales $[65,66]$, también cumple con la normalización y es una función real.

A pesar de que no podemos descartar las otras distribuciones por medio de un criterio matemático, se suele considerar a la función de Wigner como la función canónica en el espacio fase porque cumple con condiciones físicas adecuadas [60], por ello es la más cercana a la idea que tenemos de una distribución conjunta [12, 118], y además asocia a cada punto del espacio fase un observable físico [60].

Podríamos utilizar la función positivo-definida de Husimi [119], en términos de la cual se define la entropía de Wehrl. Sin embargo, nos interesa estudiar la relación de la correlación posición-momento con el principio de incertidumbre y como la función de Husimi no tiene las marginales correctas no podemos relacionar ambos conceptos. Por otro lado, nos hemos planteado estudios de la función de Husimi como una de las perspectivas de esta tesis.

\section{¿Qué significan las regiones negativas de la función de Wigner?}

La positividad de la probabilidad y de la densidad de probabilidad se garantiza axiomáticamente por construcción [120]. La función de Wigner ha motivado la discusión sobre si extender o no la teoría de las probabilidades a los números negativos, si llamarlos o no probabilidades y, en ese sentido, si darle o no una interpretación probabilista a esta distribución [121, 122].

Operacionalmente no existe diferencia entre las diferentes representaciones de la cuántica. Esto se refiere a que todos los resultados que podemos obtener con la mecánica cuántica en espacio de posición o de momento pueden obtenerse también con la función de Wigner, de hecho ésta ofrece una expresión para calcular los valores 
esperados que es análoga a como se calculan en la mecánica estadística. Entonces, operacionalmente es viable considerar probabilidades negativas sin intentar asociarlas con la ocurrencia de un observable. Pueden utilizarse como intermediarias en operaciones, tal como se realiza con números negativos en operaciones que sólo admiten como resultado un número natural [122], para preservar la congruencia en la terminología podría llamárselas probabilidades también.

En este espíritu, se ha formulado la mecánica cuántica con probabilidades extendidas [123, 124].

En otro orden de ideas, puede enfocarse el problema desde la interpretación misma de una probabilidad. La probabilidad se entiende usualmente como la frecuencia relativa de ocurrencia de un evento dentro de un conjunto de realizaciones de un experimento. Esta noción evidencia la fuerte carga experimental del concepto probabilidad. Al mismo tiempo plantea un desafío porque matemáticamente la probabilidad se consigue en el límite en el que el número de realizaciones es infinito y no se puede repetir un experimento infinitas veces. Si las probabilidades se interpretan en un sentido bayesiano, como "falta de información", es más factible pensar en que la cota inferior (cero) pueda ser removida para considerar probabilidades negativas [121].

\section{¿Es $s_{w}$ una medida de la localización de la función de Wigner?}

En el caso discreto, la condición $0 \leq p_{i} \leq 1$ implica que $s_{x} \geq 0$.

En el caso continuo la densidad de probabilidad no está acotada por arriba y si bien tenemos una cota inferior, $\rho(x)>0$, como puede tomar valores mayores que uno la entropía diferencial puede ser negativa.

En la función de Wigner la cota inferior de la probabilidades ya no es el cero y esto conduce a que la entropía de Shannon es ahora un número complejo. En el mismo orden de ideas, los números complejos también aparecen cuando se estudia la correlación cuántica entre posición y momento con la covarianza: la covarianza cuántica de dos operadores que no conmutan tiene un componente imaginario [18].

Nuestra medida de localización de la función de Wigner en el espacio fase tiene 
características similares. La interpretación de $s_{w}$ debemos hacerla estudiando ambos componentes del número complejo y la norma.

Por otro lado, la propiedad de subaditividad implica $I \geq 0$, y se prueba utilizando la convexidad del logaritmo, y esta propiedad depende de la positividad de la función de distribución de probabilidad. Por lo tanto, no puede establecerse una cota inferior a la información mutua si trabajamos con una función que asume valores negativos.

En general, las aplicaciones de las herramientas informacionales en distribuciones con regiones negativas constituyen un problema abierto. 


\section{Correlaciones estadísticas entre}

\section{las posiciones y entre los momentos en el Átomo de Moshinsky}

En este capítulo vamos a examinar el estado basal y un estado excitado del Átomo de Moshinsky. El estado basal es una gaussiana en ambos espacios (posición y momento) y el ancho de la distribución es gobernado por los potenciales.

\subsection{Estado basal}

\subsubsection{Medidas de localización}

Podemos obtener expresiones analíticas para las entropías de pares y para las de las densidades reducidas en los espacios de posición y momento,

$$
\begin{gathered}
s_{\Gamma}=1+\ln \pi-\left[\ln \omega+\frac{1}{4} \ln (2 \kappa+1)\right], \\
s_{\rho}=\frac{1}{2}(1+\ln \pi)+\frac{1}{2}\left\{-\ln 2-\ln \omega+\ln \left[\frac{1+(2 \kappa+1)^{1 / 2}}{(2 \kappa+1)^{1 / 2}}\right]\right\}, \\
s_{\Pi}=1+\ln \pi+\left[\ln \omega+\frac{1}{4} \ln (2 \kappa+1)\right],
\end{gathered}
$$




$$
s_{\pi}=\frac{1}{2}(1+\ln \pi)+\frac{1}{2}\left\{-\ln 2+\ln \omega+\ln \left[1+(2 \kappa+1)^{1 / 2}\right]\right\} .
$$

Todas las entropías dependen de ambos potenciales y están dadas en unidades naturales, nats. En el espacio de posición, las entropías de pares y las de la densidad reducida son funciones monótonamente crecientes tanto del potencial de confinamiento, $\omega$, como del potencial entre partículas, $\kappa$, en tanto que en el espacio de momento tales entropías son funciones monótonamente decrecientes de los potenciales.

En la Fig. (5.1) se muestran las gráficas de las entropías de pares como funciones de $\kappa$ para tres valores del potencial de confinamiento, $\omega=0.7,1,1.2 . s_{\Gamma}$ aumenta con la magnitud del potencial repulsivo entre partículas $(\kappa<0)$, esto es, la densidad de pares en el espacio de posición se deslocaliza conforme la intensidad de la interacción aumenta. En el espacio de momento $s_{\Pi}$ disminuye, entonces la densidad de pares se localiza conforme aumenta la interacción. El comportamiento inverso en los espacios de posición y momento puede interpretarse utilizando las relaciones de incertidumbre que deben cumplirse. Para potenciales atractivos $(\kappa>0)$ el comportamiento es opuesto al observado para potenciales repulsivos. $s_{\Gamma}$ disminuye con la magnitud del potencial, es decir, la distribución se localiza en el espacio de posición, en tanto que $s_{\Pi}$ aumenta y la distribución se deslocaliza en el espacio de momento.

El mismo tipo de comportamiento se observa para las entropías de las densidades reducidas. El punto donde cruzan las entropías, esto es donde $s_{\Gamma}=s_{\Pi}$, separa las regiones donde $s_{\Gamma}>s_{\Pi}$ (a la izquierda), y donde $s_{\Gamma}<s_{\Pi}$ (a la derecha del punto). Este punto es el mismo que el que ocurre entre las entropías de las densidades reducidas, y se caracteriza por,

$$
\kappa=\frac{1-\omega^{4}}{2 \omega^{4}}
$$

Sumando las Ecs. (5.1) y (5.3), se observa que la suma entrópica en el nivel de dos partículas no depende de ninguno de los potenciales, 

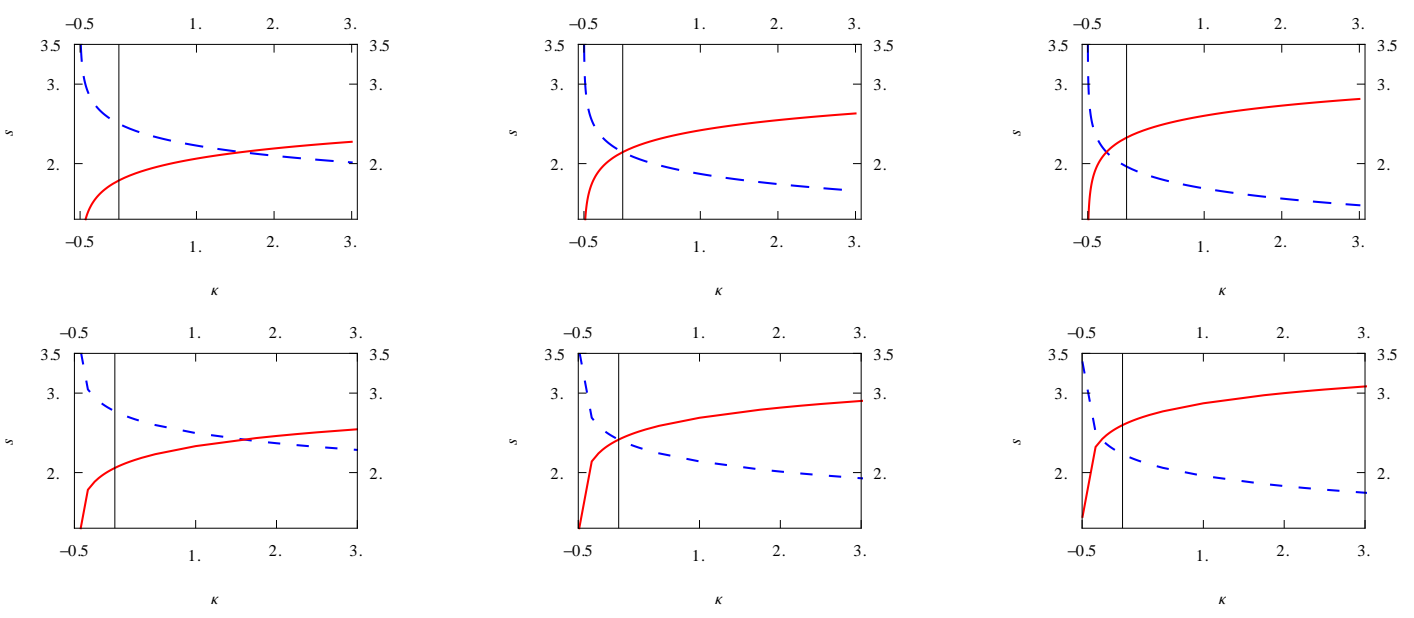

Figura 5.1: Entropías de pares, $s_{\Gamma}$ (azul, línea discontinua) y $s_{\Pi}$ (rojo, línea continua) para los estados (a) basal, $(0,0)$, y (b) excitado, $(0,1)$, como funciones del potencial entre partículas, $\kappa$, para potenciales de confinamiento $\omega=0.7,1,1.2$.

$$
s_{\Gamma}+s_{\Pi}=2(1+\ln \pi)
$$

y el valor es exactamente la cota inferior que impone la relación de incertidumbre. En tanto que si sumamos las Ecs. (5.2) y (5.4), conseguimos la expresión para la suma entrópica,

$$
s_{\rho}+s_{\pi}=1+\ln \pi-\ln 2+\ln \left[\frac{1+(2 \kappa+1)^{1 / 2}}{(2 \kappa+1)^{1 / 4}}\right],
$$

que depende del potencial entre partículas pero no del potencial de confinamiento, $\omega$. La cota inferior de esta relación de incertidumbre se alcanza cuando $\kappa=0$ y el valor aumenta para los otros valores de $\kappa$. Anteriormente se ha relacionado esta suma entrópica, calculada numéricamente, con efectos debidos a la correlación electrónica en sistemas atómicos reales, con potenciales de Coulomb [125]. La Ec. (5.7) es una expresión analítica que muestra que la suma entrópica depende de la interacción entre partículas en este modelo. 


\subsubsection{Medidas de correlación}

Pueden escribirse expresiones analíticas para el coeficiente de correlación en el estado basal en ambos espacios,

$$
\sigma_{x}=\frac{(2 \kappa+1)^{1 / 2}-1}{(2 \kappa+1)^{1 / 2}+1}=-\sigma_{p} .
$$

El coeficiente de correlación indica que la correlación entre las posiciones y entre los momentos de las partículas es de la misma magnitud, independientemente del valor de $\kappa$. Sin embargo, la correlación entre posiciones es de signo opuesto a la correlación entre momentos y esto las distingue. Debemos enfatizar que el coeficiente de correlación no depende del potencial de confinamiento, $\omega$, y solamente depende del potencial entre partículas, $\kappa$. Las gráficas correspondientes se muestran en la Fig. (5.2). Para un potencial repulsivo $(\kappa<0)$, existen correlaciones repulsivas entre las posiciones de las partículas $\left(\sigma_{x}<0\right)$, y correlaciones atractivas entre sus momentos $\left(\sigma_{p}>0\right)$. Para un potencial atractivo $(\kappa>0)$, ocurre la situación exactamente inversa. Note también que la magnitud de $\sigma$ aumenta conforme nos alejamos de $\kappa=0$, el caso no correlacionado $(\sigma=0)$.

Las relaciones para la información mutua son,

$$
I_{x}=\ln \left[\frac{1+(2 \kappa+1)^{1 / 2}}{2(2 \kappa+1)^{1 / 4}}\right]=I_{p} .
$$

De manera similar al coeficiente de correlación, la magnitud de $I_{x}$ es igual a la de $I_{p}$, y no dependen del potencial de confinamiento, $\omega$, sino solamente del potencial entre partículas, $\kappa . I_{x}$ and $I_{p}$ se muestran en la Fig. (5.3). También, la magnitud de $I$ aumenta conforme nos alejamos del caso no correlacionado, $\kappa=0$. La información mutua difiere del coeficiente de correlación en que no admite valores negativos y, por lo tanto, no podemos hacer una interpretación comparable basada en su signo. 


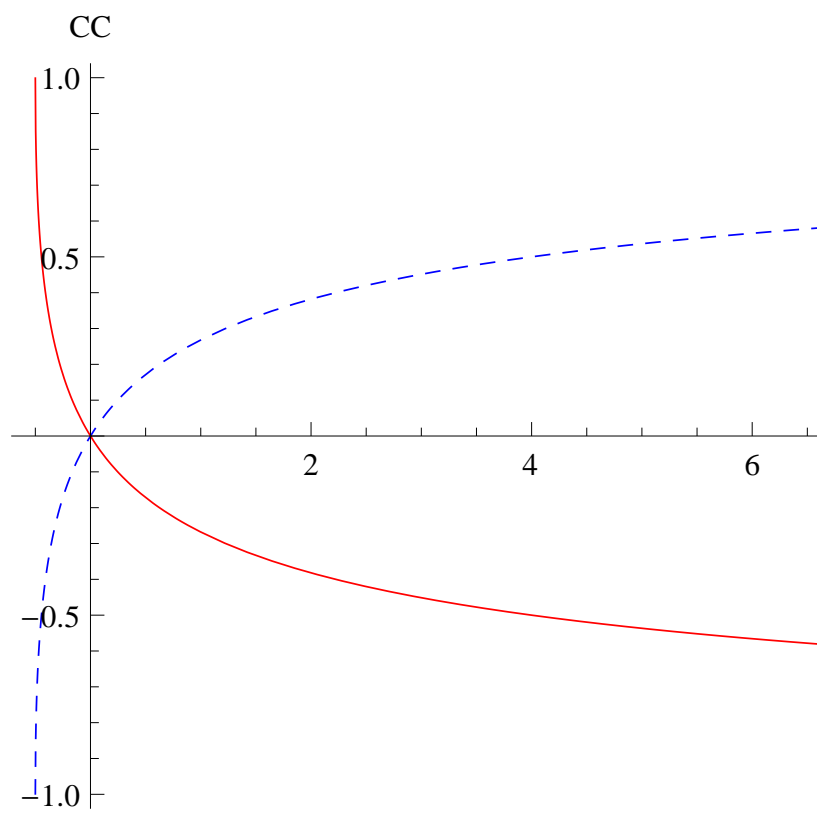

Figura 5.2: (CC) Coeficiente de correlación, $\sigma_{x}$ (azul, línea discontinua) y $\sigma_{p}$ (rojo, línea continua) para el estado basal del átomo de Moshinsky $(0,0)$, como funciones del potencial entre partículas, $\kappa$.

\section{$5.2 \quad$ Estado Excitado $\left(n_{R}, n_{r}\right)=(0,1)$}

\subsubsection{Medidas de localización}

Las entropías de los estados excitados se obtuvieron mediante la integración numérica de las Ecs. (4.45), (4.48), (4.46) y (4.49). En la Fig. (5.1b) se comparan $s_{\Gamma}$ у $s_{\Pi}$ como funciones de $\kappa$ para tres valores diferentes del potencial de confinamiento, $\omega=$ $0.7,1,1.2$, en este estado excitado. Primero, en términos generales, el comportamiento y la interpretación como funciones del potencial entre partículas es el mismo que en el estado basal. Segundo, en contraste con el estado basal las entropías del estado excitado muestran valores mayores (densidades más deslocalizadas) en ambos espacios, posición y momento.

Una característica importante es que la localización relativa (es decir, $s_{\Gamma}<s_{\Pi}$ ó $s_{\Gamma}>s_{\Pi}$ ), es controlada por ambos potenciales como en el estado basal. Hemos verificado numéricamente que los puntos en que se cruzan las entropías de posición y 


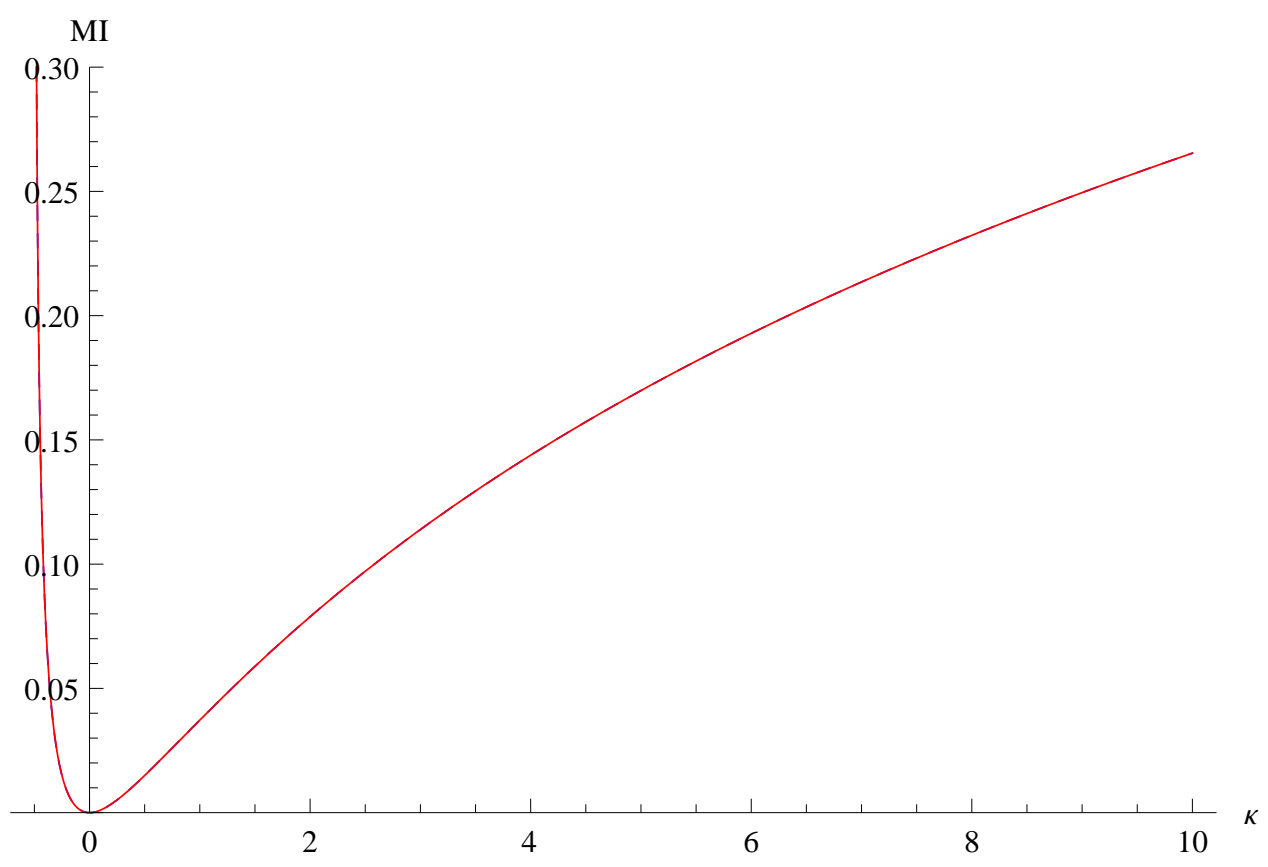

Figura 5.3: (MI) Información mutua, $I_{x}$ y $I_{p}$ para el estado basal del átomo de Moshinsky $(0,0)$, como funciones del potencial entre partículas, $\kappa$. Note que $I_{x}=I_{p}$ para este estado.

momento, caracterizados por la Ec. (5.5), son los mismos que en el estado basal (en los estados excitados esto no puede verificarse analíticamente). Lo mismo ocurre en otros estados excitados, particularmente en los casos: $\left(n_{R}, n_{r}\right)=(1,2),(1,3)$. Esto sugiere que el punto en que se cruzan (donde $s_{\Gamma}=s_{\Pi}$ ) es una función de los potenciales y no de los números cuánticos, por lo tanto es independiente del estado que nos interese. Debemos mencionar también que observamos las mismas características en el caso de las entropías de las densidades reducidas.

La simetría de la función de onda (respecto al intercambio de las variables originales, $x_{1}$ y $x_{2}$, ó $p_{1}$ y $p_{2}$ ) es controlada por $n_{r}$, el número cuántico asociado con las coordenadas relativas. Si $n_{r}$ es par, la función de onda es simétrica. Si es impar, la función de onda es antisimétrica. Por lo tanto es relevante notar que la localización relativa de las distribuciones de posición y momento parece ser controlada sólo por los potenciales. No se observan cambios cualitativos para las diferentes simetrías. La base de este argumento es que el punto en que se cruzan es el mismo para el estado basal $[(0,0)$ 
función de onda simétrica], y para el estado excitado discutido en esta sección $[(0,1)$ función de onda antisimétrica], y también para los otros estados excitados calculados $[(1,2)$ función de onda simétrica y $(1,3)$ función de onda antisimétrica].

Los resultados para la suma entrópica (obtenidos numéricamente) en los niveles de una y dos partículas son consistentes con aquellos del estado basal. La suma de las entropías de pares es constante y no depende de ninguno de los potenciales. La suma de las entropías de la densidad reducida de una partícula no depende del potencial de confinamiento, $\omega$, únicamente del potencial entre partículas, $\kappa$, y por lo tanto es sensible a las correlaciones entre ellas. Su valor aumenta conforme nos alejamos de $\kappa=0$, lo que es consistente con el estado basal. Los valores de ambas sumas son mayores en el estado excitado analizado en esta sección que en el estado basal.

Debemos enfatizar que la (in)dependencia de las entropías del potencial de confinamiento y del potencial entre partículas parece tener el mismo patrón sin importar el estado del sistema y por tanto sin importar la simetría de la función de onda.

\subsubsection{Medidas de correlación}

Los coeficientes de correlación para este estado son,

$$
\begin{gathered}
\sigma_{x}=\frac{(2 \kappa+1)^{1 / 2}-3}{(2 \kappa+1)^{1 / 2}+3}, \\
\sigma_{p}=\frac{1-3(2 \kappa+1)^{1 / 2}}{1+3(2 \kappa+1)^{1 / 2}} .
\end{gathered}
$$

Ambos coeficientes de correlación son iguales para $\kappa=0$, pero la correlación no es cero porque estamos tratando un estado excitado cuyas funciones no son separables en las variables originales $\left(x_{1} \mathrm{y} x_{2}\right.$, ó $p_{1}$ y $\left.p_{2}\right)$. Los coeficientes de correlación no dependen del potencial de confinamiento, solamente del potencial entre partículas; este comportamiento es consistente con el del estado basal.

Las gráficas de los coeficientes de correlación se muestran en la Fig. (5.4). Para un potencial repulsivo entre partículas $(\kappa<0)$ la correlación es mayor en el espacio de posición que en el de momento $\left(\left|\sigma_{x}\right|>\left|\sigma_{p}\right|\right)$, mientras que para un potencial atractivo 
entre partículas $(\kappa>0)$ la correlación es mayor en el espacio de momento $\left(\left|\sigma_{p}\right|>\left|\sigma_{x}\right|\right)$. Por lo tanto el tipo de interacción entre partículas (si ésta es atractiva o repulsiva) es el único parámetro que controla la magnitud relativa (es decir, cuál es mayor) de la correlación entre las posiciones de las partículas o entre sus momentos.

La interpretación del signo de la correlación no es tan clara como en el estado basal. Este comportamiento se resume en la Tabla (5.1). En el espacio de momento, un valor suficientemente pequeño del potencial repulsivo $\left[\kappa \in\left(-\frac{4}{9}, 0\right)\right]$ puede provocar que exista una correlación repulsiva (a diferencia de lo que pasa en el estado basal) mientras que para algunos valores del potencial atractivo $[\kappa \in(0,4)]$, existe una correlación repulsiva en el espacio de posición (lo cual también es distinto del estado basal). Debe notarse que el coeficiente de correlación es cero en el espacio de posición en $\kappa=4$ y en el espacio de momento en $\kappa=-\frac{4}{9}$, lo cual no significa de ninguna manera que no exista una correlación entre las variables en esos valores del potencial, sino que el coeficiente no es capaz de detectarla. Se puede observar en la Fig. (5.4) que la magnitud de la correlación disminuye desde la izquierda hacia esos puntos y aumenta desde esos puntos hacia la derecha. 


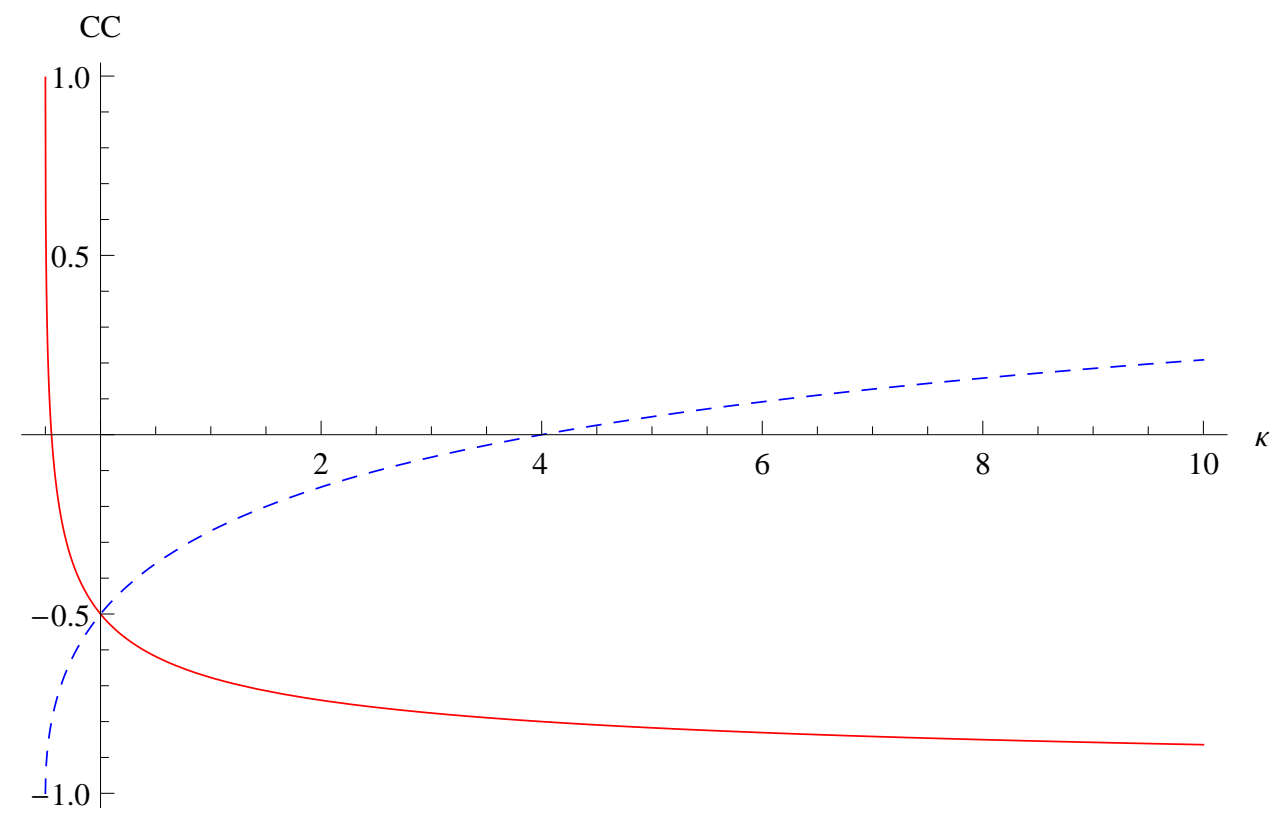

Figura 5.4: (CC) Coeficiente de correlación, $\sigma_{x}$ (azul, línea discontinua) y $\sigma_{p}$ (rojo, línea continua) para un estado excitado del átomo de Moshinsky $(0,1)$, como funciones del potencial entre partículas, $\kappa$.

Los cálculos realizados para diferentes valores del potencial de confinamiento, $\omega=$ $0.7,1,1.2,2$, muestran que la información mutua no depende en $\omega$, como en el estado basal. La Fig. (5.5) ilustra que $I_{x}>I_{p}$ si la interacción entre las partículas es repulsiva $(\kappa<0)$ en tanto que $I_{p}>I_{x}$ si ésta es atractiva $(\kappa>0)$. Esto es consistente con el comportamiento del coeficiente de correlación, que puede calcularse analíticamente para este estado. 


\begin{tabular}{|c|c|}
\hline$\kappa<0$ & $\kappa>0$ \\
\hline \multicolumn{2}{|l|}{ Coeficiente de correlación } \\
\hline$\left|\sigma_{x}\right|>\left|\sigma_{p}\right|$ & $\left|\sigma_{x}\right|<\left|\sigma_{p}\right|$ \\
\hline $\begin{array}{l}\sigma_{x}<0 \text { potencial más fuerte, } \\
\text { mayor correlación repulsiva }\end{array}$ & $\begin{array}{c}\sigma_{p}<0 \text { potencial más fuerte, } \\
\text { mayor correlación repulsiva }\end{array}$ \\
\hline $\begin{array}{c}\kappa \in\left(-\frac{1}{2},-\frac{4}{9}\right) \\
\sigma_{p}>0 \text { potencial más fuerte, } \\
\text { mayor correlación atractiva }\end{array}$ & $\begin{array}{c}\kappa \in(0,4) \\
\sigma_{x}<0 \text { potencial más fuerte, } \\
\text { menor correlación repulsiva }\end{array}$ \\
\hline $\begin{array}{c}\kappa \in\left(-\frac{4}{9}, 0\right) \\
\sigma_{p}<0 \text { potencial más fuerte, } \\
\text { menor correlación repulsiva }\end{array}$ & $\begin{array}{c}\kappa \in(4, \infty) \\
\sigma_{x}>0 \text { potencial más fuerte, } \\
\text { mayor correlación atractiva }\end{array}$ \\
\hline \multicolumn{2}{|l|}{ Información mutua } \\
\hline$I_{x}>I_{p}$ & $I_{x}<I_{p}$ \\
\hline $\begin{array}{c}I_{x} \text { potencial más fuerte, } \\
\text { mayor correlación }\end{array}$ & $\begin{array}{c}I_{p} \text { potencial más fuerte, } \\
\text { mayor correlación }\end{array}$ \\
\hline $\begin{array}{c}\quad \kappa \in\left(-\frac{1}{2},-0.43\right) \\
I_{p} \text { potencial más fuerte, } \\
\text { mayor correlación }\end{array}$ & $\begin{array}{l}\quad \kappa \in(0,3.33) \\
I_{x} \text { potencial más fuerte, } \\
\text { menor correlación }\end{array}$ \\
\hline $\begin{array}{c}\quad \kappa \in(-0.43,0) \\
I_{p} \text { potencial más fuerte, } \\
\text { menor correlación }\end{array}$ & $\begin{array}{c}\quad \kappa \in(3.33, \infty) \\
I_{x} \text { potencial más fuerte, } \\
\text { mayor correlación }\end{array}$ \\
\hline
\end{tabular}

Tabla 5.1: Análisis del coeficiente de correlación y de la información mutua para el estado excitado del átomo de Moshinsky $(0,1)$. 


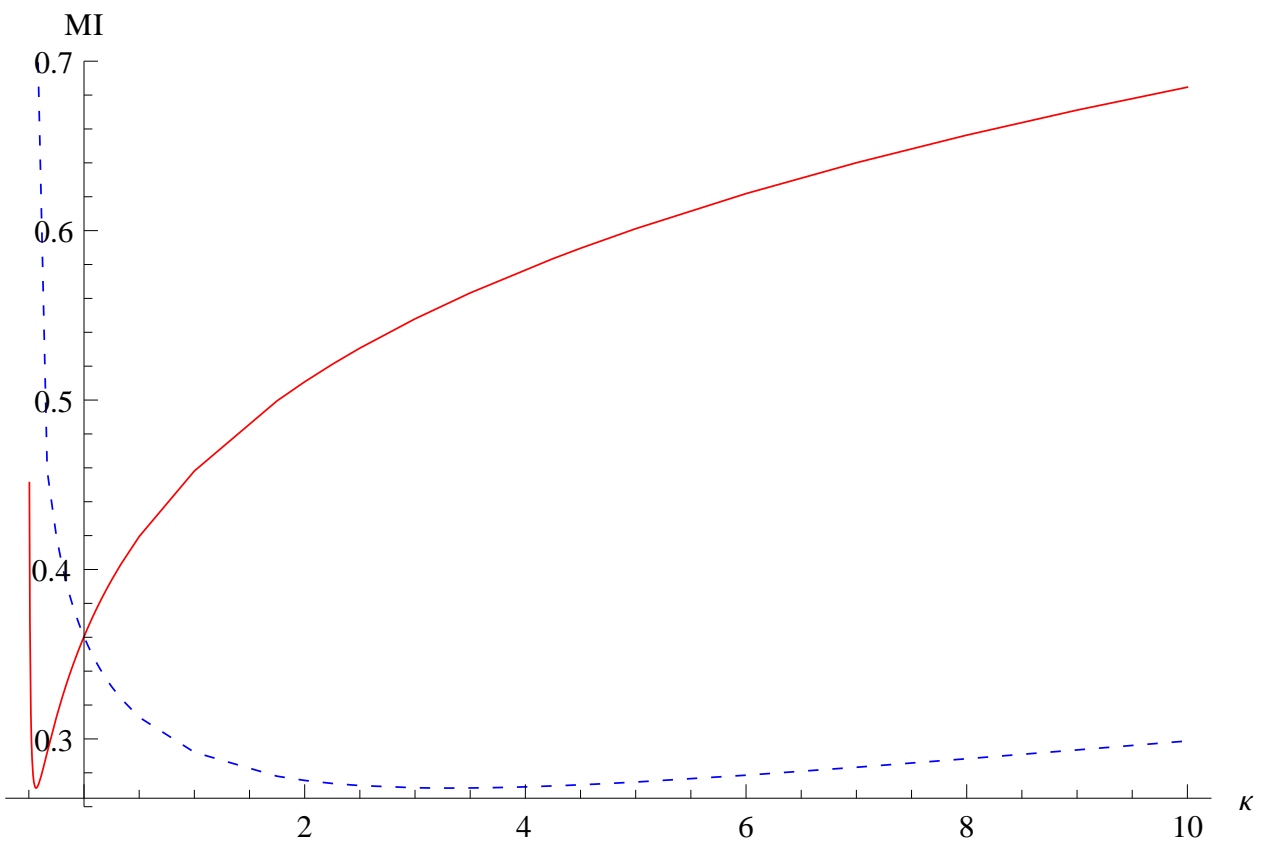

Figura 5.5: (MI) Información mutua, $I_{x}$ (azul, línea discontinua) y $I_{p}$ (rojo, línea continua) para un estado excitado del átomo de Moshinsky $(0,1)$, como funciones del potencial entre partículas, $\kappa$.

Existen dos mínimos a partir de los cuales la correlación aumenta en ambos espacios (hacia la izquierda o hacia la derecha), la interpretación de estos puntos es similar a la que hicimos para los ceros del coeficiente de correlación. Los mínimos se encuentran en, $\kappa \approx 3.33$ en el espacio de posición y en $\kappa \approx-0.43$ en el espacio de momento. En la Fig. (5.6) se muestra un análisis gráfico de estas dos regiones donde los mínimos se observan claramente. Queremos destacar que estos puntos no coinciden con los ceros en el coeficiente de correlación ( $\kappa=4$ en espacio- $x$ y $\kappa=-\frac{4}{9}$ en espacio- $p$ ) y que los mínimos no son cero en la información mutua. Por otra parte, en los valores del potencial en los que el coeficiente de correlación es cero, la información mutua no lo es. En dichos puntos la información mutua es capaz de detectar correlación en el sistema mientras que el coeficiente de correlación no lo es. Por otra parte, calculamos la información mutua para otros estados excitados $\left(n_{R}, n_{r}\right)=(1,2),(1,3)$ y en ellos se observa un comportamiento similar. 

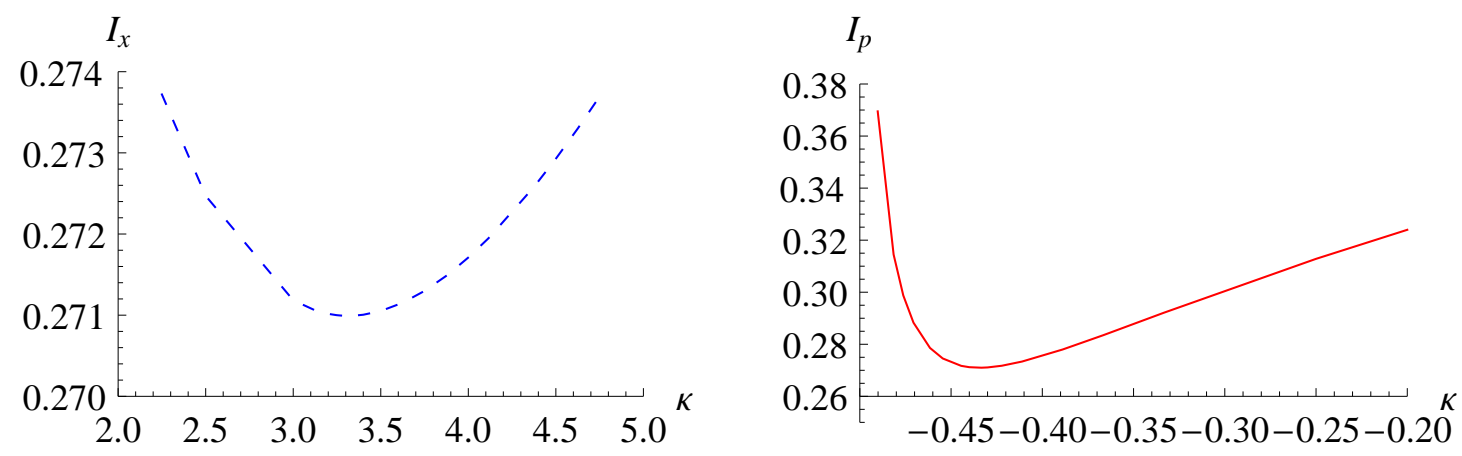

Figura 5.6: Análisis de las regiones en la información mutua, $I_{x}$ (izquierda, azul, línea discontinua) y $I_{p}$ (derecha, rojo, línea continua), para un estado excitado del átomo de Moshinsky $(0,1)$, que contienen los puntos donde la correlación aumenta cuando se varía el potencial entre partículas, $\kappa$.

\subsection{Conclusiones}

Utilizamos las entropías de Shannon para examinar la localización de las distribuciones de dos partículas y de las densidades reducidas del átomo de Moshinsky en los espacios de posición, momento y espacio fase en el estado basal y en algunos estados excitados. El comportamiento de las entropías se examinó como función del potencial de confinamiento, $\omega$, y del potencial entre partículas, $\kappa$.

El punto en el que se cruzan las entropías de ambos espacios depende de los potenciales y es independiente del estado y de la simetría de la función de onda.

La suma entrópica del estado basal depende explícitamente del potencial entre partículas, lo que demuestra su sensibilidad a las correlaciones entre partículas. Resultados numéricos también demuestran esta sensibilidad en estados excitados.

Hemos examinado las medidas de correlación y encontramos que son independientes del potencial de confinaminento, $\omega$, y sólo dependen del potencial entre partículas, $\kappa$. En el estado basal, la magnitud de la correlación entre las posiciones y entre los momentos es igual. 
En estados excitados, la magnitud de la correlación es mayor entre los momentos de las partículas que entre sus posiciones si éstas interactúan a través de un potencial atractivo. Cuando interactúan a través de un potencial repulsivo, las correlaciones entre las posiciones son mayores que entre los momentos. 


\section{Simetría en la función de onda,}

\section{huecos de simetría, interacciones y correlación estadística}

En un sistema cuántico de más de una partícula y cuando éstas son idénticas, las interacciones entre ellas son gobernadas por el potencial y por la simetría en la función de onda. La simetría en la función de onda es una restricción que se impone para garantizar que tales partículas sean indistinguibles. La indistinguibilidad implica que el tratamiento estadístico de cada partícula es equivalente.

En este capítulo discutiremos la influencia mutua entre la simetría de la función de onda y el potencial a través del estudio de la localización de las partículas y de la correlación entre ellas en el átomo de Moshinsky. El análisis se puede realizar en el espacio de posición o en el de momento.

Dado que hay dos regímenes posibles de potencial, podemos analizar la influencia que el signo de la interacción ejerce sobre la localización de las partículas y sobre la correlación estadística en un estado con una cierta simetría. A partir de esto, el tipo de preguntas que nos podemos plantear son ¿Si el potencial es repulsivo (como en el caso electrónico) qué distribución es más localizada, la simétrica o la antisimétrica? ¿Y en cuál de estos estados es mayor la correlación entre las partículas? Y si variara la intensidad del potencial ¿podría ésta inducir cambios? Es decir, invertir el orden relativo de la localización y de la correlación por medio de variar el potencial. La 
simetría en la función de onda tiene un impacto en la localización de las distribuciones y en la correlación entre partículas.

Si fuera posible fijar todos los factores que influyen en la localización y en la corrrelación en un sistema y mantenerlos iguales para una función simétrica y una antisimétrica, esperaríamos que la distribución que proviene de la función antisimétrica estuviese más localizada que la de la función simétrica. La razón es que en un sistema de dos partículas, la posición de una de ellas (o del momento), restringe la posición (o el momento) de la otra, que puede adoptar todos excepto ese si su dinámica es gobernada por una función antisimétrica. Esto es consecuencia de que no pueden estar en la misma posición, $\Psi_{\text {antisim }}(x, x)=0$, ni tener el mismo valor del momento, $\Psi_{\text {antisim }}(p, p)=0$, en tanto que en general $\Psi_{\text {sim }}(x, x) \neq 0$ ó $\Psi_{\text {sim }}(p, p) \neq 0$.

Por la misma razón esperaríamos que la correlación entre las partículas fuera mayor si el estado de éstas se representa por una función antisimétrica.

La simetría en la función de onda, por lo general, provoca que las funciones no sean separables en funciones de una partícula. El potencial también evita que las soluciones de una ecuación de Schrödinger de varias partículas puedan escribirse como producto de funciones de una sola partícula.

Hemos analizado antes la influencia que tiene la simetría en la localización y en la correlación $[2,3]$ en un sistema de dos partículas no interactuantes. Si no hay un potencial entre las partículas se puede encontrar una solución separable y simetrizarla para conseguir un estado simétrico o antisimétrico de dos partículas indistinguibles. Sin embargo, cuando existe un potencial entre las partículas las soluciones no pueden escribirse de forma separable y simplemente simetrizarse, sino que la no separabilidad que proviene del potencial se mezcla con la no separabilidad que proviene de la simetría en la función de onda, de manera que para estudiar la mutua influencia debemos diseñar un modelo adecuado.

El átomo de Moshinsky es un sistema analítico en el cuál podemos controlar la simetría de la función de onda a través de variar uno de los números cuánticos, lo cual puede aprovecharse para diseñar un esquema en que puede estudiarse la influencia del 
potencial y la simetría.

En este capítulo pretendemos ampliar algunos de los resultados que pueden encontrarse en la literatura. Estudiaremos el "Hueco de Moshisnky" 1, que ha sido analizado previamente [38, 126, 127, 56] añadiendo en nuestro anális el hueco de Fermi, con énfasis en la manera en que ambos huecos se influyen y tomando en consideración potenciales repulsivos y atractivos. Recientemente se ha reportado una simetría entre las medidas de entrelazado en este modelo: cuando se intercambian las escalas energéticas del centro de masa y de las coordenadas relativas, el entrelazado es invariante [29, 35]; para algunos estados explicamos este comportamiento considerando el hueco de contrapeso que se ha observado en otros sistemas [128, 129].

\subsection{Diseño del modelo}

Por lo antes explicado, no es posible fijar el resto de los factores que influyen y permitir que sólo el potencial y la simetría sean variables. Si esto fuera posible tendríamos un par de funciones del tipo:

$$
\begin{aligned}
& \Psi_{a}\left(x_{1}, x_{2}\right)=\phi_{1}\left(x_{1}\right) \phi_{2}\left(x_{2}\right)-\phi_{2}\left(x_{1}\right) \phi_{1}\left(x_{2}\right) \\
& \Psi_{s}\left(x_{1}, x_{2}\right)=\phi_{1}\left(x_{1}\right) \phi_{2}\left(x_{2}\right)+\phi_{2}\left(x_{1}\right) \phi_{1}\left(x_{2}\right)
\end{aligned}
$$

es decir, soluciones que son separables y que simetrizamos como si se tratara de dos partículas no interactuantes en una caja [3] o de dos osciladores no interactuantes. Con esto se podrían estudiar las diferencias entre ambas funciones atendiendo a la localización o la correlación de las partículas mientras varía el potencial entre ellas. Escribir las funciones de esta forma implicaría que:

$$
\hat{H}=\frac{{\hat{p_{1}}}^{2}}{2 m}+\frac{{\hat{p_{2}}}^{2}}{2 m}+V\left(x_{1}\right)+V\left(x_{2}\right),
$$

\footnotetext{
${ }^{1}$ Que es el análogo al hueco de Coulomb en los potenciales armónicos
} 
pero si tenemos un potencial entre partículas en el Hamiltoniano $\left(V\left(x_{1}, x_{2}\right)\right)$, la solución exacta no es separable en las coordenadas (o en los momentos) de cada una de ellas. Así, la no separabilidad de la función de onda se debe tanto al potencial como a la simetría.

Para este capítulo, es relevante que la simetría de la función de onda en el átomo de Moshinsky puede controlarse con el número cuántico asociado con las coordenadas relativas $\left(r=x_{1}-x_{2}\right.$ ó $\left.p=p_{1}-p_{2}\right)$. La razón es que la paridad del polinomio de Hermite $\left[H_{n_{r}}(r)\right]$ está dada por su orden: a $n_{r}$ par (impar) corresponde un polinomio par (impar). Si intercambiamos las coordenadas de las partículas $\left(r^{\prime}=x_{2}-x_{1}=-r\right)$ y si $n_{r}$ es par, entonces $H_{n_{r}}(r)=H_{n_{r}}(-r)$, por lo tanto la función de onda es simétrica. En cambio si $n_{r}$ es impar, entonces $H_{n_{r}}(r)=-H_{n_{r}}(-r)$, por lo tanto la función de onda es antisimétrica. La magnitud de la interacción entre las partículas (gobernada por $\lambda / \omega)$ puede manipularse variando la magnitud del potencial de la trampa $(\omega)$.

Recordemos que la energía de un estado arbitrario está dada por,

$$
E_{n_{R}, n_{r}}(\omega, \lambda)=\omega\left(n_{R}+\frac{1}{2}\right)+\sqrt{\omega^{2} \pm 2 \lambda^{2}}\left(n_{r}+\frac{1}{2}\right)
$$

por lo tanto, en el límite no interactuante, $\omega \rightarrow \infty$, si se cumple que $n_{R_{1}}+n_{r_{1}}=$ $n_{R_{2}}+n_{r_{2}}$, los estados $\left(n_{R_{1}}, n_{r_{1}}\right)$ y $\left(n_{R_{2}}, n_{r_{2}}\right)$ son degenerados y sus funciones de onda están dadas por,

$$
\begin{aligned}
& \Psi\left(x_{1}, x_{2}\right)=\phi_{n_{R_{1}}}\left(x_{1}\right) \phi_{n_{r_{1}}}\left(x_{2}\right) \pm \phi_{n_{r_{1}}}\left(x_{1}\right) \phi_{n_{R_{1}}}\left(x_{2}\right) \\
& \Psi\left(x_{1}, x_{2}\right)=\phi_{n_{R_{2}}}\left(x_{1}\right) \phi_{n_{r_{2}}}\left(x_{2}\right) \pm \phi_{n_{r_{2}}}\left(x_{1}\right) \phi_{n_{R_{2}}}\left(x_{2}\right)
\end{aligned}
$$

los signos, y por tanto la simetría bajo el intercambio, dependen de la paridad del polinomio de Hermite asociado con las coordenadas relativas.

En casos donde dos estados son degenerados en el límite no interactuante podemos tener funciones de onda que sólo difieren en la simetría, así, "prender" el potencial puede dar indicios idea de su influencia en la simetría del sistema. 
También existen casos de estados degenerados en el límite no interactuante que además de diferir en la simetría lo hacen también en los números cuánticos involucrados, pues la única condición para la degeneración es que $n_{R_{1}}+n_{r_{1}}=n_{R_{2}}+n_{r_{2}}$. Por tal razón hay estados que no difieren en la simetría pero sí lo hacen en los números cuánticos. La caracterización de la localización y de la correlación en los estados debería tener en cuenta los diferentes casos.

En las secciones siguientes analizaremos parejas de estados con diferente simetría que son degenerados en el límite no interactuante. Aunque estamos interesados en enfatizar las comparaciones entre diferentes simetrías, para completar el análisis del modelo, dedicaremos una sección a estudiar los estados cuyas funciones de onda tienen la misma simetría y son degenerados en ese límite.

Asumimos que $n_{R}, n_{r}=0,1,2,3$, por lo tanto las parejas de estados que analizaremos son:

- $[(0,1),(1,0)]$.

- $[(1,1),(2,0)],[(1,1),(0,2)] \mathrm{y}[(2,0),(0,2)]$.

- $[(0,3),(3,0)],[(2,1),(1,2)],[(0,3),(1,2)],[(2,1),(3,0)],[(0,3),(2,1)] \mathrm{y}[(3,0),(1,2)]$.

- $[(1,3),(3,1)],[(3,1),(2,2)] \mathrm{y}[(1,3),(2,2)]$.

- $[(2,3),(3,2)]$.

\subsection{Simetría de la función de onda y huecos de simetría}

Primero vamos a analizar los casos en que sólo están intercambiados los números cuánticos en funciones de diferente simetría. Los estados que analizaremos en esta sección serán $[(0,1),(1,0)],[(0,3),(3,0)],[(2,1),(1,2)]$ y $[(2,3),(3,2)]$. 
En el límite no interactuante las funciones de onda tendrán la forma,

$$
\begin{gathered}
\Psi_{21}\left(x_{1}, x_{2}\right)=\psi_{2}\left(x_{1}\right) \psi_{1}\left(x_{2}\right)-\psi_{1}\left(x_{1}\right) \psi_{2}\left(x_{2}\right) \\
\Psi_{12}\left(x_{1}, x_{2}\right)=\psi_{2}\left(x_{1}\right) \psi_{1}\left(x_{2}\right)+\psi_{1}\left(x_{1}\right) \psi_{2}\left(x_{2}\right) .
\end{gathered}
$$

hemos tomado como ejemplo el caso $[(2,1),(1,2)]$. Debe notarse que ambas funciones sólo intercambian los números cuánticos, $n_{R}$ y $n_{r}$. Esto, sumado a que la única diferencia entre estas funciones es que una es simétrica y la otra antisimétrica, obliga a que alguno de los números cuánticos sea necesariamente impar y el otro sea par. Estas funciones pueden entenderse como formadas por "orbitales". Intercambiando los números cuánticos podemos conseguir para el estado una función simétrica o una antisimétrica en el límite no interactuante, con los mismos orbitales ocupados. Todas las funciones de onda tratadas en esta sección se reducen a formas similares.

En otra parte del capítulo se discutirán los casos $[(2,0),(0,2)]$ y $[(1,3),(3,1)]$ en que el intercambio de los números cuánticos no altera la simetría de la función de onda.

A continuación analizaremos la localización de las partículas con la entropía de Shannon de la densidad de pares, para posteriormente estudiar la entropía de Shannon de la densidad reducida de una variable, y para finalmente tratar la correlación estadística en el sistema estudiando la información mutua. En todos los casos discutiremos los espacios de posición y momento.

\subsubsection{Localización en la densidad de pares}

La Tabla (6.1) compara las diferentes medidas de localización y correlación para los estados $[(2,1),(1,2)]$. A primera vista se advierte que las entropías de pares son iguales para ambos estados, esto es, $s_{\Gamma_{21}}=s_{\Gamma_{12}}$ y $s_{\Pi_{21}}=s_{\Pi_{12}}$, lo que quiere decir que se encuentran igualmente localizados o deslocalizados. De donde se desprende que la suma entrópica también es igual para ambos estados, $s_{T_{21}}=s_{T_{12}}$.

El estado simétrico y el antisimétrico tienen valores iguales de las entropías de pares, sin importar si nos fijamos en el espacio de posición $\left(s_{\Gamma}\right)$, en el de momento $\left(s_{\Pi}\right)$, o en el 


\begin{tabular}{|ccc|}
\hline $\begin{array}{c}(2,1),(1,2) \\
\text { Atractivo }\end{array}$ & No interactuante & Repulsivo \\
\hline$E_{21}<E_{12}$ & $E_{21}=E_{12}$ & $E_{21}>E_{12}$ \\
\hline$s_{\Gamma_{21}}=s_{\Gamma_{12}}$ & $s_{\Gamma_{21}}=s_{\Gamma_{12}}$ & $s_{\Gamma_{21}}=s_{\Gamma_{12}}$ \\
\hline$s_{\rho_{21}}>s_{\rho_{12}}$ & $s_{\rho_{21}}=s_{\rho_{12}}$ & $s_{\rho_{21}}<s_{\rho_{12}}$ \\
\hline$I_{x_{21}}>I_{x_{12}}$ & $I_{x_{21}}=I_{x_{12}}$ & $I_{x_{21}}<I_{x_{12}}$ \\
\hline$s_{\Pi_{21}}=s_{\Pi_{12}}$ & $s_{\Pi_{21}}=s_{\Pi_{12}}$ & $s_{\Pi_{21}}=s_{\Pi_{12}}$ \\
\hline$s_{\pi_{21}}<s_{\pi_{12}}$ & $s_{\pi_{21}}=s_{\pi_{12}}$ & $s_{\pi_{21}}>s_{\pi_{12}}$ \\
\hline$I_{p_{21}}<I_{p_{12}}$ & $I_{p_{21}}=I_{p_{12}}$ & $I_{p_{21}}>I_{p_{12}}$ \\
\hline$s_{T_{21}}=s_{T_{12}}$ & $s_{T_{21}}=s_{T_{12}}$ & $s_{T_{21}}=s_{T_{12}}$ \\
\hline$s_{t_{21}}=s_{t_{12}}$ & $s_{t_{21}}=s_{t_{12}}$ & $s_{t_{21}}=s_{t_{12}}$ \\
\hline$I_{t_{21}}=I_{t_{12}}$ & $I_{t_{21}}=I_{t_{12}}$ & $I_{t_{21}}=I_{t_{12}}$ \\
\hline
\end{tabular}

Tabla 6.1: Comparación de los estados $(2,1)$ y $(1,2)$.

espacio fase separable $\left(s_{T}\right)$, como puede observarse en la Fig. (6.1), donde mostramos las entropías para $\lambda=0.5$.

La función de onda antisimétrica tiene la restricción del hueco de Fermi en tanto que la función simétrica no la tiene. ¿Cómo podemos explicar entonces que la simetría no sea un factor en la localización de las partículas en ambos estados? 

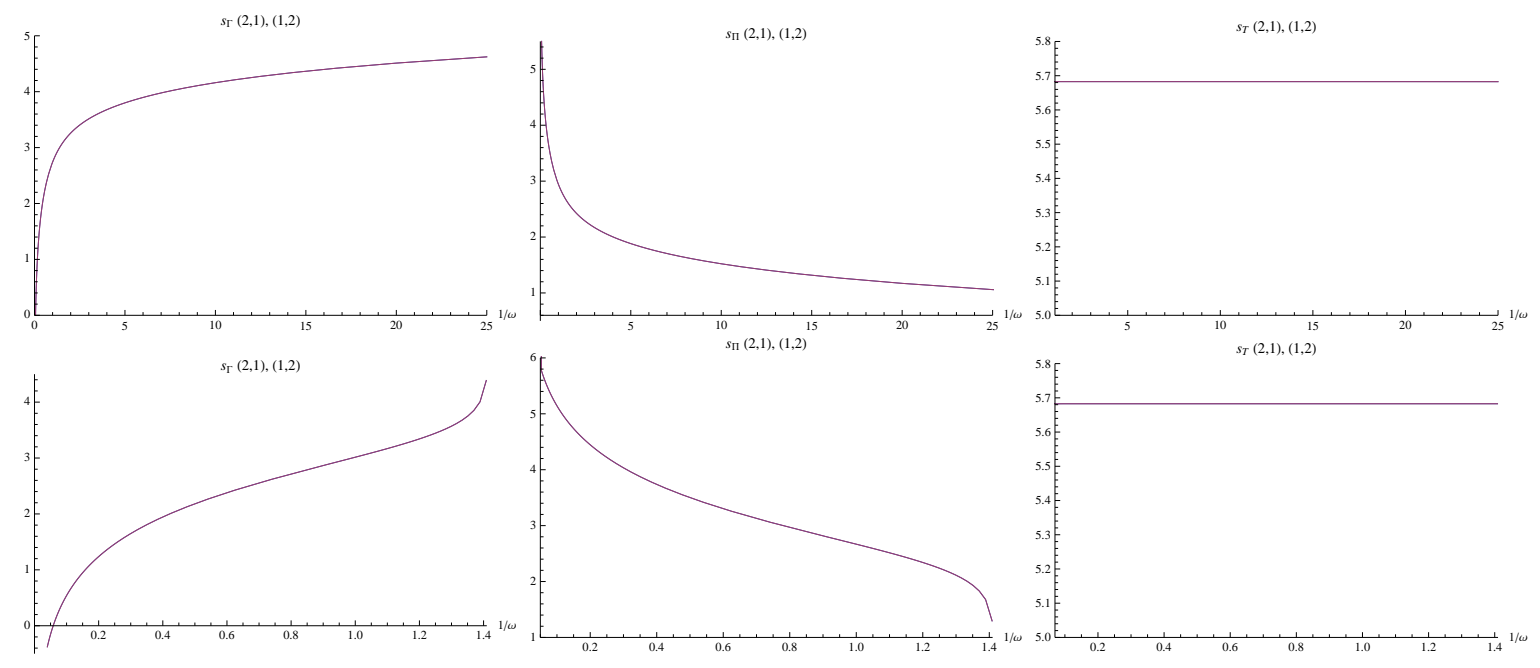

Figura 6.1: Entropías de pares para los estados $[(2,1),(1,2)]$ con potencial atractivo (arriba) y con potencial repulsivo (abajo), con $\lambda=0.5$.

La presencia del hueco de Fermi afecta la localización de la densidad porque si $x_{1}=x_{2}=x$, entonces $\Psi_{21}(x, x)=0$, es decir, para cada $x_{1}=x$, la otra partícula puede estar en todo el espacio excepto en $x_{2}=x$, esperamos que la densidad sea más localizada si comparamos con el caso donde no existe tal restricción. Sin embargo, la función simétrica del ejemplo (y todas las de este capítulo) tiene otro tipo de hueco. Este hueco de "contrapeso" ha sido observado y reportado antes para sistemas atómicos [128, 129] y se manifiesta a través de una restricción sobre la función de onda, y por tanto sobre las densidades, cuando las partículas se encuentran a la misma distancia del origen. Si $x_{1}=-x_{2}$, entonces $x_{1}=x$, nos conduce a $\Psi_{12}(x,-x)=0$.

El hueco de Fermi está presente en todos los estados con $n_{r}$ impar. Por su parte, si $n_{R}$ es impar existirá en la función un hueco de contrapeso. La presencia de los huecos está asociada con la presencia de los polinomios de Hermite como puede inferirse a partir la forma de las funciones. Enfatizamos que el hueco de Fermi está asociado con las coordenadas relativas y el hueco de contrapeso con las coordenadas del centro de masa.

En esta sección alguno de los dos números cuánticos será impar porque la diferente 
simetría se debe a que sólo intercambiamos $n_{r}$ y $n_{R}$, por lo que siempre habrá uno de los dos huecos en las densidades.

El resultado anterior permite concluir que las entropías de dos partículas no distinguen entre un hueco de Fermi y un hueco de contrapeso, sea en el espacio de posición o en el de momento y en cualquier régimen de interacción (atractivo o repulsivo). Los dos tipos de hueco influyen de la misma manera en la entropía y por lo tanto afectan la localización de las partículas en la misma medida.

En la Fig. (6.2) se grafican las densidades de pares de los estados $[(2,1),(1,2)]$ en los espacios de posición y momento para potenciales repulsivos y atractivos. Se puede observar la presencia del hueco de Fermi en el estado $(2,1)$ sobre las líneas $x_{1}=x_{2} \mathrm{y}$ $p_{1}=p_{2}$, donde $\Gamma$ y $\Pi$ son cero. De manera similar, el hueco de contrapeso en el estado $(1,2)$, cuando $x_{1}=-x_{2}$ y $p_{1}=-p_{2}$ también es muy notorio.

Ahora nos enfocaremos en el comportamiento de las entropías como funciones del potencial de confinamiento. En la Fig. (6.1) se muestran las entropías de Shannon como funciones de $1 / \omega$ en los espacios de posición, momento y en el espacio fase separable. El límite no interactuante se alcanza cuando el potencial de confinamiento tiende a infinito y domina por encima del potencial entre partículas, $1 / \omega \rightarrow 0$. La interacción entre partículas aumenta a lo largo del eje $x$ conforme la intensidad de la trampa armónica decrece.

En la Tabla (6.1) puede observarse que la igualdad entre las entropías de pares de los diferentes estados es independiente de la magnitud del potencial de confinamiento $\omega$.

La densidad de pares en el espacio de posición se deslocaliza (la entropía de pares aumenta) conforme el potencial de confinamiento disminuye. Esta deslocalización está presente en ambos regímenes del potencial entre partículas como puede observarse en la Fig. (6.1). 

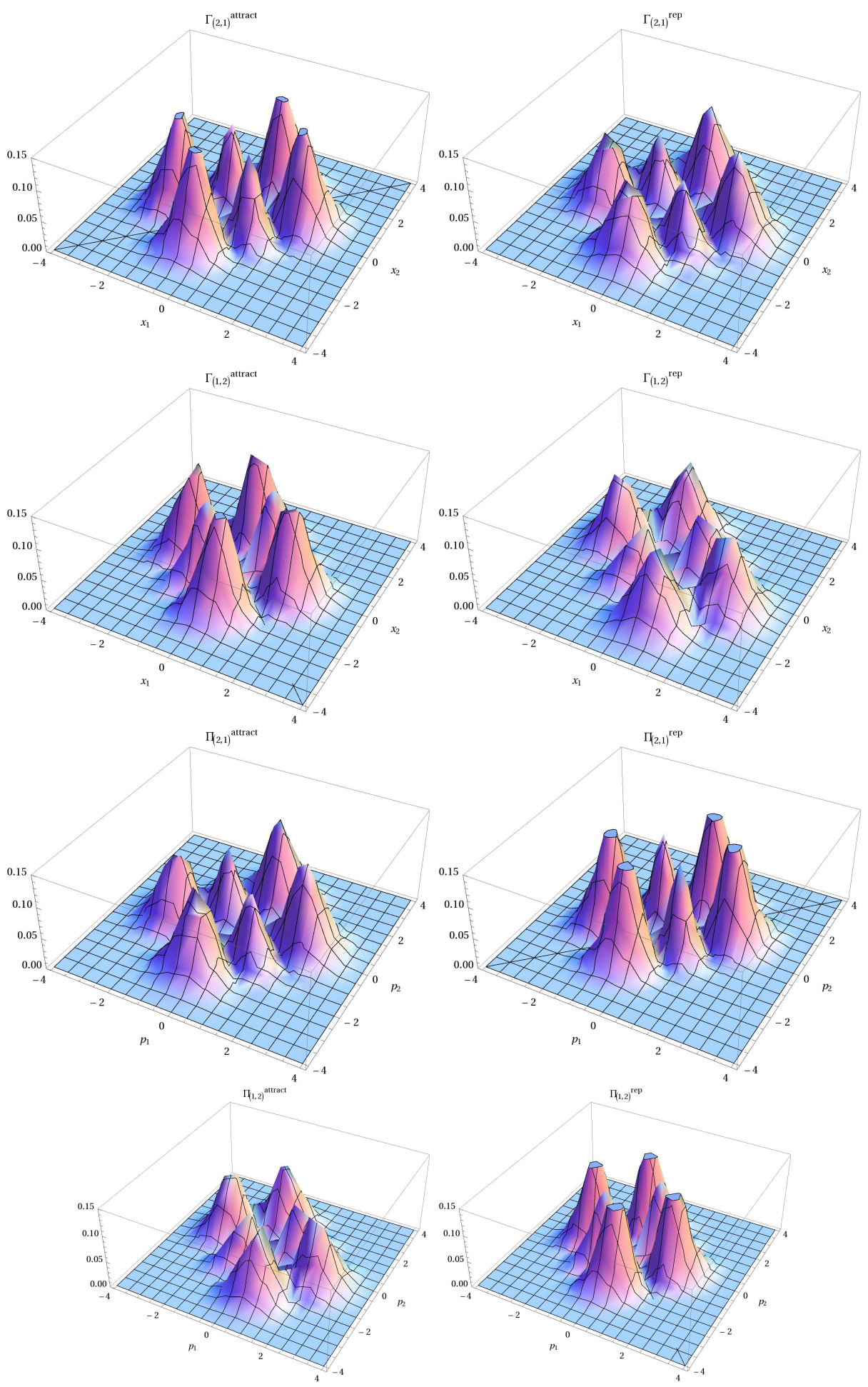

Figura 6.2: Densidades de pares para los estados $[(2,1),(1,2)]$ con potencial atractivo (columna izquierda) y con potencial repulsivo (columna derecha), con $\lambda=0.5$ y $\omega=1$. 
No obstante existe una diferencia evidente en las regiones donde $\omega$ es pequeña, es decir las regiones de confinamiento ligero donde el potencial entre partículas juega un papel muy importante. Observamos que la curva para el potencial repulsivo aumenta de una forma más pronunciada si se le compara con la correspondiente al potencial atractivo. Este comportamiento demuestra que además de la disminución de la fuerza de la trampa el potencial repulsivo es otro factor importante de deslocalización en espacio de posición porque obliga a las partículas a apartarse. Con el potencial atractivo el factor dominante en la deslocalización es la disminución de la fuerza de la trampa.

En el espacio de momento pueden observarse características comparables. La densidad se localiza (las entropías de pares disminuyen) conforme 1/ $\omega$ aumenta. Este comportamiento es consistente con la deslocalización en el espacio de posición y puede interpretarse como proveniente de las limitaciones que imponen las relaciones de incertidumbre. Con el potencial repulsivo la localización de la densidad es más abrupta que con el atractivo, como puede apreciarse en la Fig. (6.1). Esto es análogo a lo observado en el espacio de posición.

Finalmente, debe notarse que el compromiso entre la deslocalización en el espacio de posición y la localización en el espacio de momento es tal que las sumas entrópicas son constantes en ambos regímenes del potencial. De hecho, el valor de la suma entrópica no cambia si nos alejamos del límite no interactuante. Queremos subrayar la insensibilidad al potencial de la suma entrópica de dos partículas. Hacemos notar también que la suma entrópica toma valores por encima de la cota para $s_{T}$, que es $2(1+\ln \pi) \approx 4.29$.

Estos resultados también son válidos para las parejas de estados $[(0,1),(1,0)]$, $[(0,3),(3,0)], \mathrm{y}[(2,3),(3,2)]$, como puede advertirse en las Tablas $(6.2),(6.3)$ y $(6.4)$. 


\begin{tabular}{|ccc|}
\hline $\begin{array}{c}(0,1),(1,0) \\
\text { Atractivo }\end{array}$ & No interactuante & Repulsivo \\
\hline$E_{01}>E_{10}$ & $E_{01}=E_{10}$ & $E_{01}<E_{10}$ \\
\hline$s_{\Gamma_{01}}=s_{\Gamma_{10}}$ & $s_{\Gamma_{01}}=s_{\Gamma_{10}}$ & $s_{\Gamma_{01}}=s_{\Gamma_{10}}$ \\
\hline$s_{\rho_{01}}<s_{\rho_{10}}$ & $s_{\rho_{01}}=s_{\rho_{10}}$ & $s_{\rho_{01}}>s_{\rho_{10}}$ \\
\hline$I_{x_{01}}<I_{x_{10}}$ & $I_{x_{01}}=I_{x_{10}}$ & $I_{x_{01}}>I_{x_{10}}$ \\
\hline$s_{\Pi_{01}}=s_{\Pi_{10}}$ & $s_{\Pi_{01}}=s_{\Pi_{10}}$ & $s_{\Pi_{01}}=s_{\Pi_{10}}$ \\
\hline$s_{\pi_{01}}>s_{\pi_{10}}$ & $s_{\pi_{01}}=s_{\pi_{10}}$ & $s_{\pi_{01}}<s_{\pi_{10}}$ \\
\hline$I_{p_{01}}>I_{p_{10}}$ & $I_{p_{01}}=I_{p_{10}}$ & $I_{p_{01}}<I_{p_{10}}$ \\
\hline$s_{T_{01}}=s_{T_{10}}$ & $s_{T_{01}}=s_{T_{10}}$ & $s_{T_{01}}=s_{T_{10}}$ \\
\hline$s_{t_{01}}=s_{t_{10}}$ & $s_{t_{01}}=s_{t_{10}}$ & $s_{t_{01}}=s_{t_{10}}$ \\
\hline$I_{t_{01}}=I_{t_{10}}$ & $I_{t_{01}}=I_{t_{10}}$ & $I_{t_{01}}=I_{t_{10}}$ \\
\hline
\end{tabular}

Tabla 6.2: Comparación de los estados $(0,1)$ y $(1,0)$. 


\begin{tabular}{|ccc|}
\hline $\begin{array}{c}(0,3),(3,0) \\
\text { Atractivo }\end{array}$ & No interactuante & Repulsivo \\
\hline$E_{03}>E_{30}$ & $E_{03}=E_{30}$ & $E_{03}<E_{30}$ \\
\hline$s_{\Gamma_{03}}=s_{\Gamma_{30}}$ & $s_{\Gamma_{03}}=s_{\Gamma_{30}}$ & $s_{\Gamma_{03}}=s_{\Gamma_{30}}$ \\
\hline$s_{\rho_{03}}<s_{\rho_{30}}$ & $s_{\rho_{03}}=s_{\rho_{30}}$ & $s_{\rho_{03}}>s_{\rho_{30}}$ \\
\hline$I_{x_{03}}<I_{x_{30}}$ & $I_{x_{03}}=I_{x_{30}}$ & $I_{x_{03}}>I_{x_{30}}$ \\
\hline$s_{\Pi_{03}}=s_{\Pi_{30}}$ & $s_{\Pi_{03}}=s_{\Pi_{30}}$ & $s_{\Pi_{03}}=s_{\Pi_{30}}$ \\
\hline$s_{\pi_{03}}>s_{\pi_{30}}$ & $s_{\pi_{03}}=s_{\pi_{30}}$ & $s_{\pi_{03}}<s_{\pi_{30}}$ \\
\hline$I_{p_{03}}>I_{p_{30}}$ & $I_{p_{03}}=I_{p_{30}}$ & $I_{p_{03}}<I_{p_{30}}$ \\
\hline$s_{T_{03}}=s_{T_{30}}$ & $s_{T_{03}}=s_{T_{30}}$ & $s_{T_{03}}=s_{T_{30}}$ \\
\hline$s_{t_{03}}=s_{t_{30}}$ & $s_{t_{03}}=s_{t_{30}}$ & $s_{t_{03}}=s_{t_{30}}$ \\
\hline$I_{t_{03}}=I_{t_{30}}$ & $I_{t_{03}}=I_{t_{30}}$ & $I_{t_{03}}=I_{t_{30}}$ \\
\hline
\end{tabular}

Tabla 6.3: Comparación de los estados $(0,3)$ y $(3,0)$. 


\begin{tabular}{|ccl|}
\hline $\begin{array}{c}(2,3),(3,2) \\
\text { Atractivo }\end{array}$ & No interactuante & Repulsivo \\
\hline$E_{23}>E_{32}$ & $E_{23}=E_{32}$ & $E_{23}<E_{32}$ \\
\hline$s_{\Gamma_{23}}=s_{\Gamma_{32}}$ & $s_{\Gamma_{23}}=s_{\Gamma_{32}}$ & $s_{\Gamma_{23}}=s_{\Gamma_{32}}$ \\
\hline$s_{\rho_{23}}<s_{\rho_{32}}$ & $s_{\rho_{23}}=s_{\rho_{32}}$ & $s_{\rho_{23}}>s_{\rho_{32}}$ \\
\hline$I_{x_{23}}<I_{x_{32}}$ & $I_{x_{23}}=I_{x_{32}}$ & $I_{x_{23}}>I_{x_{32}}$ \\
\hline$s_{\Pi_{23}}=s_{\Pi_{32}}$ & $s_{\Pi_{23}}=s_{\Pi_{32}}$ & $s_{\Pi_{23}}=s_{\Pi_{32}}$ \\
\hline$s_{\pi_{23}}>s_{\pi_{32}}$ & $s_{\pi_{23}}=s_{\pi_{32}}$ & $s_{\pi_{23}}<s_{\pi_{32}}$ \\
\hline$I_{p_{23}}>I_{p_{32}}$ & $I_{p_{23}}=I_{p_{32}}$ & $I_{p_{23}}<I_{p_{32}}$ \\
\hline$s_{T_{23}}=s_{T_{32}}$ & $s_{T_{23}}=s_{T_{32}}$ & $s_{T_{23}}=s_{T_{32}}$ \\
\hline$s_{t_{23}}=s_{t_{32}}$ & $s_{t_{23}}=s_{t_{32}}$ & $s_{t_{23}}=s_{t_{32}}$ \\
\hline$I_{t_{23}}=I_{t_{32}}$ & $I_{t_{23}}=I_{t_{32}}$ & $I_{t_{23}}=I_{t_{32}}$ \\
\hline
\end{tabular}

Tabla 6.4: Comparación de los estados $(2,3)$ y $(3,2)$.

\subsubsection{Localización en la densidad reducida de una variable}

Las entropías correspondientes a las densidades reducidas de una partícula que se observan en las Tablas (6.2), (6.3) y (6.4) muestran un comportamiento muy diferente comparadas con las entropías de pares. En ellas los efectos de la simetría son diferentes en cada espacio, de hecho, son opuestos en cualquiera de los regímenes del potencial.

Para el análisis retomaremos el ejemplo $[(2,1),(1,2)]$. Para potenciales atractivos en el espacio de posición observamos que $s_{\rho_{21}}>s_{\rho_{12}}$. En el espacio de momento ocurre la situación opuesta, $s_{\pi_{21}}<s_{\pi_{12}}$. Esta oposición en los signos puede entenderse si se nota que $s_{t_{21}}=s_{t_{12}}$.

En este caso, la densidad que proviene de la función antisimétrica (con el hueco de Fermi) se encuentra más localizada en el espacio de momento que la que proviene de la función simétrica (con el hueco de contrapeso). Por otro lado, la densidad proveniente de la función simétrica está más localizada que la antisimétrica en el espacio de posición. 

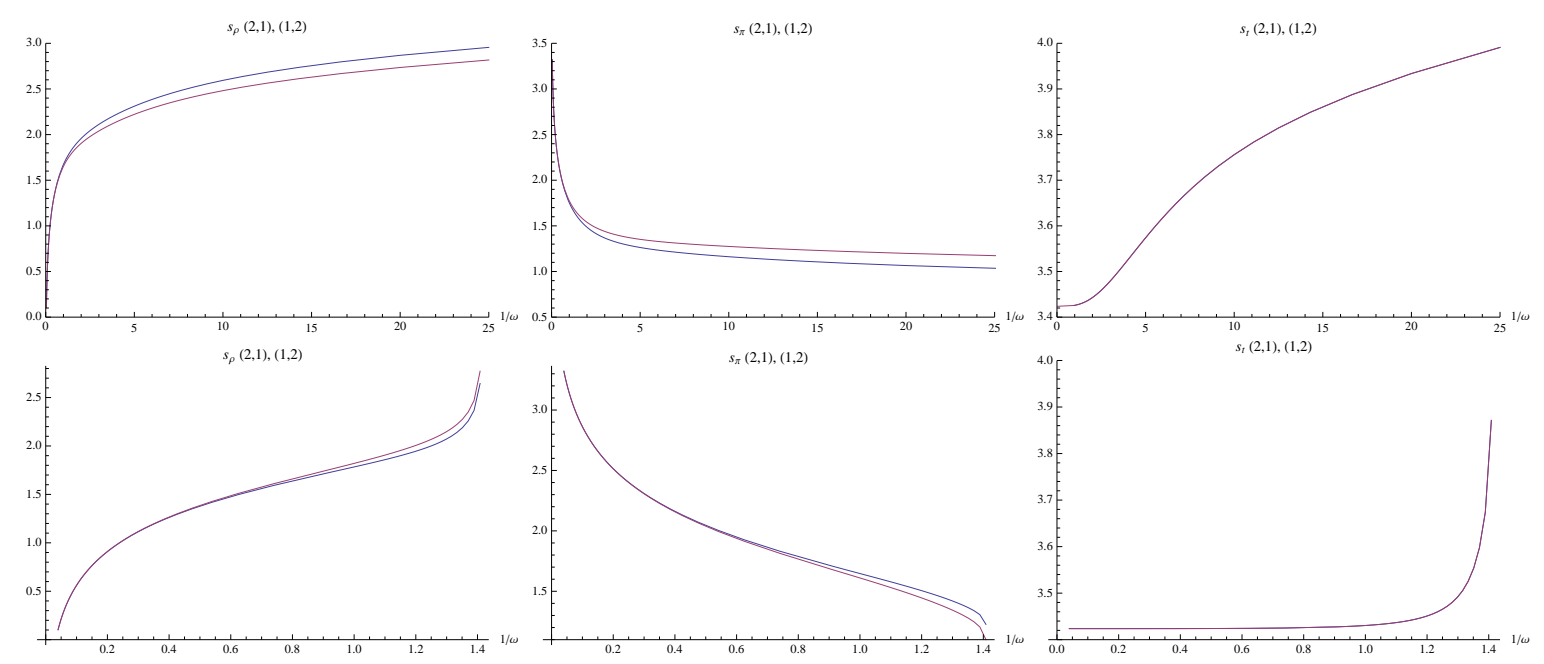

Figura 6.3: Entropías de la densidad reducida de una partícula para los estados $(2,1)$ [azul], y $(1,2)$ [rojo] con potencial atractivo (arriba) y potencial repulsivo (abajo), con $\lambda=0.5$.

Por su parte, la suma entrópica, $s_{t}$, que mide la incertidumbre en el espacio fase separable, no detecta estos cambios en la simetría, es decir tenemos $s_{t_{21}}=s_{t_{12}}$. En la Fig. (6.3) se muestra que estos resultados son independientes del régimen del potencial que estemos tratando.

De la forma de las funciones de onda en el límite no interactuante puede desprenderse que $\rho_{21}(x)=\rho_{12}(x)$ y $\pi_{21}(p)=\pi_{12}(p)$, consecuentemente $s_{\rho_{21}}=s_{\rho_{12}}$ y $s_{\pi_{21}}=s_{\pi_{12}}$.

Considerando el potencial repulsivo tenemos que $s_{\rho_{21}}<s_{\rho_{12}}$ y la situación contraria en el espacio de momento, es decir, $s_{\pi_{21}}>s_{\pi_{12}}$. La densidad reducida que proviene de la función antisimétrica está más localizada que la que proviene de la simétrica en el espacio de posición y más deslocalizada en el espacio de momento. Los signos entre las entropías son exactamente opuestos si consideramos el potencial atractivo. Pero en ambos casos tenemos que $s_{t_{21}}=s_{t_{12}}$.

Dado que el signo entre las entropías es diferente para cada régimen de potencial (considere por ejemplo las $s_{\rho}$ 's en ambos regímenes), podemos argumentar que el orden de éstas nos permite distinguir entre las densidades reducidas que provienen de funciones de onda con diferente simetría. Para reforzar nuestro argumento, en la Fig. 
(6.3) se muestra que conforme aumenta la magnitud del potencial las curvas se separan cada vez más.

Tal comportamiento es interesante porque el distinguir entre funciones de diferente simetría, asociadas a partículas bajo un potencial se conoce como el problema de la $v$-representabilidad en otros contextos, como DFT [130].

Las relaciones precisas entre las entropías para el resto de los estados se observan claramente si se inspeccionan las Tablas (6.2), (6.3) y (6.4), las que muestran que en todos los casos ocurre esta oposición de los signos entre los espacios.

A continuación hacemos un análisis detallado de los signos opuestos de las entropías de las densidades reducidas mostrados en la Tabla (6.1). Para ello, primero analizaremos los signos opuestos entre las energías de los dos estados. En el límite no interactuante, tenemos que $E_{21}=E_{12}$, en tanto que bajo un potencial atractivo tenemos que $E_{21}<E_{12}$, y exactamente el caso opuesto bajo un potencial repulsivo, es decir $E_{21}>E_{12}$.

Si tomamos la diferencia en energía entre dos estados arbitrarios encontramos,

$$
\begin{aligned}
\frac{E_{n_{R}, n_{r}}-E_{m_{R}, m_{r}}}{\omega}= & {\left[\left(n_{R}+\frac{1}{2}\right)+\sqrt{1 \pm 2 \frac{\lambda^{2}}{\omega^{2}}}\left(n_{r}+\frac{1}{2}\right)\right]-} \\
& {\left[\left(m_{R}+\frac{1}{2}\right)+\sqrt{1 \pm 2 \frac{\lambda^{2}}{\omega^{2}}}\left(m_{r}+\frac{1}{2}\right)\right] } \\
= & \left(n_{R}-m_{R}\right)+\sqrt{1 \pm 2 \frac{\lambda^{2}}{\omega^{2}}}\left(n_{r}-m_{r}\right) .
\end{aligned}
$$

Para el análisis nos hemos restringido en este capítulo a los casos donde $\left(n_{R}+n_{r}\right)=$ $\left(m_{R}+m_{r}\right)$, esto es, $\left(n_{R}-m_{R}\right)=\left(m_{r}-n_{r}\right)$. Esta condición se satisface automáticamente si consideramos estados que difieren solamente por el intercambio de sus número cuánticos, es decir, $n_{R}=m_{r}$ y $m_{R}=n_{r}$. Reordenando la ecuación de arriba encontramos

$$
\frac{E_{n_{R}, n_{r}}-E_{m_{R}, m_{r}}}{\omega}=\left(\sqrt{1 \pm 2 \frac{\lambda^{2}}{\omega^{2}}}-1\right)\left(n_{r}-m_{r}\right) .
$$

La existencia de degeneraciones en el límite no interactuante $(\omega \rightarrow \infty)$ es evidente. Ahora dividiremos en casos. Para un potencial atractivo tenemos que

$$
\frac{\Delta E}{\omega}=\frac{E_{n_{R}, n_{r}}-E_{m_{R}, m_{r}}}{\omega}=\left(\sqrt{1+2 \frac{\lambda^{2}}{\omega^{2}}}-1\right)\left(n_{r}-m_{r}\right) .
$$


El factor que multiplica la diferencia de los números cuánticos siempre es positivo. Concluimos entonces que el signo de $\Delta E$ es gobernado por la diferencia entre $n_{r}$ y $m_{r}$, o entre $n_{R}$ y $m_{R}$, debido a las restricciones sobre su suma. Entonces,

$$
\begin{array}{llll}
\Delta E>0 & \text { si } & n_{r}>m_{r}, & n_{R}<m_{R} \\
\Delta E<0 & \text { si } & n_{r}<m_{r}, & n_{R}>m_{R} .
\end{array}
$$

Para un potencial repulsivo, tenemos

$$
\frac{\Delta E}{\omega}=\frac{E_{n_{R}, n_{r}}-E_{m_{R}, m_{r}}}{\omega}=\left(\sqrt{1-2 \frac{\lambda^{2}}{\omega^{2}}}-1\right)\left(n_{r}-m_{r}\right) .
$$

El factor arriba mencionado es siempre negativo y el signo de $\Delta E$ es opuesto al del caso atractivo.

El signo entre las energías es opuesto al signo entre las correspondientes entropías de Shannon en el espacio de posición y es el mismo que entre las entropías en el espacio de momento. Así, si $E_{1}>E_{2}$, entonces $s_{\rho_{1}}<s_{\rho_{2}}$ y $s_{\pi_{1}}>s_{\pi_{2}}$ como puede observarse en las Tablas (6.1), (6.2), (6.3) y (6.4).

Ahora utilizaremos que, en virtud del teorema virial, para este modelo $\bar{T}=\bar{V}$ [43]. Entonces, $E_{1}>E_{2}$ implica $\bar{T}_{1}>\bar{T}_{2}$ y también $\bar{V}_{1}>\bar{V}_{2}$.

Si tomamos el caso en que $\bar{T}_{1}>\bar{T}_{2}$ se esperaría que un incremento en la energía cinética condujera a un incremento en el momento promedio de las partículas. La distribución de momentos debería entonces ensancharse y deslocalizarse en el caso con mayor $\bar{T}$, lo que resultaría en $s_{\pi_{1}}>s_{\pi_{2}}$.

Por otro lado, $\bar{V}_{1}>\bar{V}_{2}$ implica que el potencial de confinamiento es mayor en el primer caso. Es plausible entonces que la densidad se localice en el caso con mayor $\bar{V}$ y tener $s_{\rho_{1}}<s_{\rho_{2}}$.

Estos efectos pueden apreciarse en las gráficas de las densidades reducidas presentadas en la Fig. (6.4). En el espacio de momento se observa una disminución de la densidad alrededor del origen en el estado con mayor energía comparado con el otro, en ambos regímenes del potencial. Puesto que ambos estados tienen densidades con la misma normalización la consecuencia debe ser una transferencia de densidad a valores mayores de $|p|$. En el espacio de posición se ve una concentración de la densidad 
alrededor del origen en los estados con mayor energía, esta situación es caracterizada entrópicamente como una localización; en los estados con menor energía la remoción de densidad hacia regiones lejanas al origen provoca deslocalización.

La magnitud de los números cuánticos es un factor que influye en el ordenamiento de las energías y por tanto en la localización de las distribuciones. Los casos $(0,1),(1,0)$; $(0,3),(3,0) ; \mathrm{y}(2,3),(3,2)$ arrojan resultados similares como puede observarse en las Tablas (6.2), (6.3) y (6.4).

Los argumentos anteriores pueden utilizarse para explicar por qué el orden de las curvas en la Fig. (6.3) es diferente según la naturaleza del potencial. Para comprender el orden hay que atender al ordenamiento energético para un potencial repulsivo y para uno atractivo. Además, es importante señalar que para intensidades grandes del potencial repulsivo existe una localización más pronunciada en el espacio de momento y una deslocalización más pronunciada en espacio de posición si se compara con el potencial atractivo, lo cual es similar a lo observado en las entropías de pares.

Por su parte, las medidas de localización en el espacio fase separable, $s_{t}$, no distinguen entre el hueco de Fermi y el de contrapeso. Sin embargo, sí dependen de la magnitud del potencial entre partículas, en contraste con $s_{T}$. $s_{t}$ se incrementa con la magnitud del potencial en ambos regímenes. El valor de $s_{t}$ para el límite no interactuante está por encima de la cota $1+\ln \pi$. 


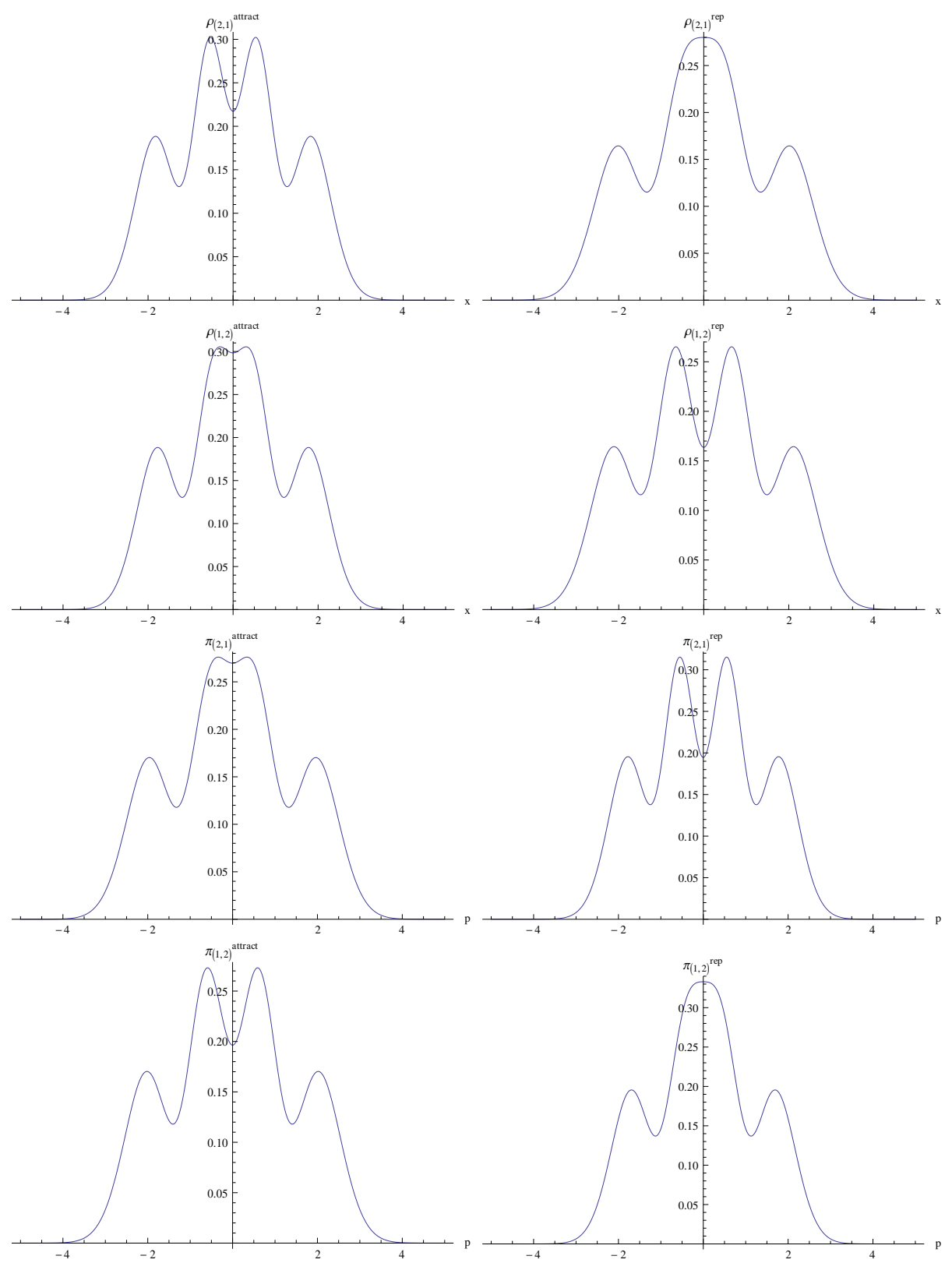

Figura 6.4: Densidades reducidas de una partícula para los estados $[(2,1),(1,2)]$ con un potencial atractivo (columna izquierda) y con un potencial repulsivo (columna derecha), con $\omega=1$ у $\lambda=0.5$. 


\subsubsection{Correlación estadística}

La correlación estadística medida a través de la información mutua es un balance entre la localización de la densidad de dos partículas y de la densidad reducida. En los casos tratados en esta sección el orden relativo de la información mutua para estados de diferente simetría está determinado por el orden de las entropías de Shannon de las densidades reducidas porque las entropías de pares son iguales. El estado con una densidad reducida más deslocalizada corresponde al estado con mayor correlación.

En la Fig. (6.5) se presentan las gráficas correspondientes a la información mutua para el par de estados $[(2,1),(1,2)]$. El estado antisimétrico, que tiene el hueco de Fermi, está más correlacionado en el espacio de posición que el simétrico, que tiene el hueco de contrapeso, con un potencial atractivo. Por otro lado, el estado simétrico es el más correlacionado en el espacio de momento. Con un potencial repulsivo la situación es la inversa. En el estado antisimétrico las partículas están más correlacionadas en el espacio de momento en tanto que en el estado simétrico están más correlacionadas en el espacio de posición.

Como un rasgo general la información mutua muestra una tendencia creciente conforme 1/ $\omega$ aumenta. Sin embargo, se observan mínimos en varios casos conforme la magnitud del potencial varía. Los mínimos pueden caracterizarse según el siguiente esquema: Si $n_{r}>n_{R}$, y el potencial es atractivo, entonces $I_{x}$ tendrá un mínimo y si el potencial es repulsivo habrá un mínimo en $I_{p}$. En cambio si $n_{r}<n_{R}$, habrá un mínimo en $I_{x}$ si el potencial es repulsivo y en $I_{p}$ si es atractivo.

Para explicar estos mínimos en las medidas de correlación utilizaremos el balance que existe entre la localización en la densidad de pares y en la densidad reducida. Para ejemplificar consideremos la situación en el espacio de posición, donde $s_{\Gamma}$ y $s_{\rho}$ aumentan conforme aumenta $1 / \omega$. Si la información mutua tiene un mínimo, debemos concluir que $s_{\Gamma}$ exhibe una deslocalización más pronunciada comparada con la que exhibe $s_{\rho}$ en la región previa al mínimo y por lo tanto la correlación disminuye. La región posterior al mínimo, donde la correlación aumenta, está mostrando que la deslocalización en $\rho$ 
es más pronunciada que la deslocalización en $\Gamma$ con la variación de $\omega$. Estas diferencias se deben al efecto del potencial. Estos resultados demuestran que el potencial afecta de manera distinta a la densidad de pares y a la densidad reducida y esta diferencia se refleja en las medidas de localización lo que sólo es evidente examinando las medidas de correlación.
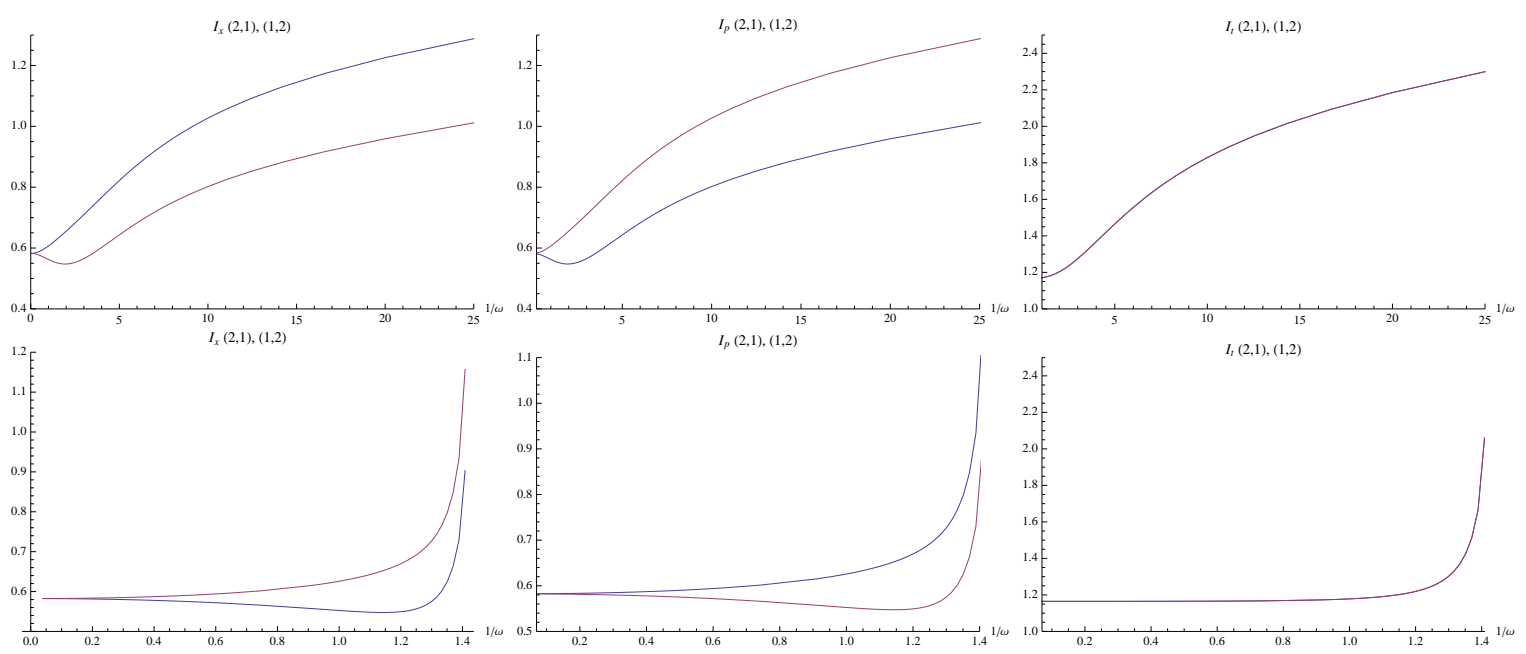

Figura 6.5: Información mutua para los estados $(2,1)$ [azul] y $(1,2)$ [rojo] con potencial atractivo (arriba) y potencial repulsivo (abajo), con $\lambda=0.5$.

La suma de las correlaciones en los espacios de posición y momento, $I_{t}$, no distingue entre los estados simétricos y los antisimétricos ya que sus componentes, $s_{T}$ y $s_{t}$, no lo hacen. Su comportamiento como función del potencial es el mismo que el de $s_{t}$ porque $s_{T}$ es constante. Para los casos atractivo y repulsivo $I_{t}$ aumenta con la magnitud del potencial, de manera similar a $s_{t}$.

Para poner en perspectiva estos resultados es necesario señalar que el comportamiento es consistente con el observado para los estados singulete y triplete de la serie isoelectrónica del helio donde $s_{t}$ e $I_{t}$ aumentan para $Z$ pequeño (correlación repulsiva grande) [131]. Por otro lado la suma entrópica, $s_{t}$, ha sido propuesta como una medida de correlación en sistemas atómicos [125], sobre la base de resultados obtenidos numéricamente. El estudio de este modelo ilustra que $s_{t}$ (proveniente de la densidad 
reducida) tiene el mismo comportamiento que la suma de $I_{x}$ e $I_{p}$ que son medidas de correlación relacionadas con la densidad de pares. Los resultados obtenidos en sistemas atómicos reales muestran tendencias consistentes con los obtenidos para este modelo que utiliza potenciales armónicos.

\subsection{Simetría en la función de onda, huecos de simetría y números cuánticos}

En esta sección también vamos a comparar las propiedades de una función antisimétrica con las de una función simétrica. En contraste con la sección anterior, elegimos estados en que las partículas ocupan diferente orbitales en el límite no interactuante. Nuestro análisis se enfoca en los efectos del potencial conforme nos alejamos de este límite. La única restricción sobre estos estados es que sean degenerados en el límite no interactuante, esto es, deben satisfacer $\left(n_{R}+n_{r}\right)=\left(m_{R}+m_{r}\right)$. Los pares que consideramos en esta sección son $[(1,1),(2,0)] ;[(1,1),(0,2)] ;[(0,3),(1,2)] ;[(2,1),(3,0)] ;[(3,1),(2,2)]$ y $[(1,3),(2,2)]$. Usaremos el par $[(2,1),(3,0)]$ para ilustrar el comportamiento general. Las funciones de onda en el límite no interactuante son,

$$
\begin{aligned}
& \Psi_{21}\left(x_{1}, x_{2}\right)=\psi_{1}\left(x_{1}\right) \psi_{2}\left(x_{2}\right)-\psi_{2}\left(x_{1}\right) \psi_{1}\left(x_{2}\right) \\
& \Psi_{30}\left(x_{1}, x_{2}\right)=\psi_{0}\left(x_{1}\right) \psi_{3}\left(x_{2}\right)+\psi_{3}\left(x_{1}\right) \psi_{0}\left(x_{2}\right) .
\end{aligned}
$$

Ya que las partículas están en diferentes orbitales en ambos estados el orden relativo de las entropías y de las informaciones mutuas en el límite no interactuante está determinado por los números cuánticos; para el caso que discutiremos más ampliamente el orden relativo de las medidas en este límite se muestra en la Tabla (6.5).

Para completar el análisis del modelo también mostramos el resumen de los resultados de los otros estados en las Tablas (6.6), (6.7), (6.8), (6.9) y (6.10).

Existen características comunes a todas las tablas. Una notable es que en todos los casos existen cruces en las medidas de localización y en las de correlación en alguno 
de los regímenes de potencial. Otra característica importante es que todas las Tablas contienen cruces en $I_{t}$, la correlación total, en ambos regímenes del potencial.

La caracterización de los cruces en las medidas de correlación y de localización se sintetizan como sigue:

- $[(2,1),(3,0)]$ y $[(1,1),(2,0)]$. Tablas $(6.5)$ y $(6.6)$.

- Potencial atractivo. Cruces en $s_{\rho}$ e $I_{p}$.

- Potencial repulsivo. Cruces en $s_{\pi}$ e $I_{x}$.

- $[(1,1),(0,2)]$ y $[(0,3),(1,2)]$. Tablas $(6.7)$ y $(6.8)$.

- Potencial atractivo. Cruces en $s_{\pi}$ e $I_{x}$.

- Potencial repulsivo. Cruces en $s_{\rho}$ e $I_{p}$.

- $[(3,1),(2,2)]$. Tabla $(6.9)$.

- Potencial atractivo. Cruces en $s_{\pi}, I_{p}$ y $s_{t}$.

- Potencial repulsivo. Cruces en $s_{\rho}, I_{x}$ y $s_{t}$.

- $[(1,3),(2,2)]$. Tabla $(6.10)$.

- Potencial atractivo. Cruces en $s_{\rho}, I_{x}$ y $s_{t}$.

- Potencial repulsivo. Cruces en $s_{\pi}, I_{p}$ y $s_{t}$. 


\begin{tabular}{|ccc|}
\hline $\begin{array}{c}(2,1),(3,0) \\
\text { Atractivo }\end{array}$ & No interactuante & Repulsivo \\
\hline$E_{21}>E_{30}$ & $E_{21}=E_{30}$ & $E_{21}<E_{30}$ \\
\hline$s_{\Gamma_{21}}>s_{\Gamma_{30}}$ & $s_{\Gamma_{21}}>s_{\Gamma_{30}}$ & $s_{\Gamma_{21}}>s_{\Gamma_{30}}$ \\
\hline$s_{\rho_{21}}>s_{\rho_{30}(<2.55)}$ & $s_{\rho_{21}}>s_{\rho_{30}}$ & $s_{\rho_{21}}>s_{\rho_{30}}$ \\
$s_{\rho_{21}}<s_{\rho_{30}}(>2.55)$ & & \\
\hline$I_{x_{21}}<I_{x_{30}}$ & $I_{x_{21}}<I_{x_{30}}$ & $I_{x_{21}}<I_{x_{30}}(<1.18)$ \\
& & $I_{x_{21}}>I_{x_{30}}(>1.18)$ \\
\hline$s_{\Pi_{21}}>s_{\Pi_{30}}$ & $s_{\Pi_{21}}>s_{\Pi_{30}}$ & $s_{\Pi_{21}}>s_{\Pi_{30}}$ \\
\hline$s_{\pi_{21}}>s_{\pi_{30}}$ & $s_{\pi_{21}}>s_{\pi_{30}}$ & $s_{\pi_{21}}>s_{\pi_{30}}(<1.23)$ \\
& & $s_{\pi_{21}}<s_{\pi_{30}}(>1.23)$ \\
\hline$I_{p_{21}}<I_{p_{30}}(<2.12)$ & $I_{p_{21}}<I_{p_{30}}$ & $I_{p_{21}}<I_{p_{30}}$ \\
$I_{p_{21}}>I_{p_{30}}(>2.12)$ & & $I_{t_{21}}>I_{t_{30}}(>1.36)$ \\
\hline$s_{T_{21}}>s_{T_{30}}$ & $s_{T_{21}}>s_{T_{30}}(<1.36)$ \\
\hline$s_{t_{21}}>s_{t_{30}}$ & $s_{t_{21}}>s_{t_{30}}$ & $s_{t_{21}}>s_{t_{30}}$ \\
\hline$I_{t_{21}}<I_{t_{30}}(<4.45)$ & $I_{t_{21}}<I_{t_{30}}$ \\
$I_{t_{21}}>I_{t_{30}}(>4.45)$ & & $I_{t_{2}}$ \\
\hline
\end{tabular}

Tabla 6.5: Comparación entre los estados $(2,1)$ y $(3,0)$. Los valores de $1 / \omega$ para los puntos de cruce se indican entre paréntesis y son aproximados. 


\begin{tabular}{|ccc|}
\hline $\begin{array}{c}(1,1),(2,0) \\
\text { Atractivo }\end{array}$ & No interactuante & Repulsivo \\
\hline$E_{11}>E_{20}$ & $E_{11}=E_{20}$ & $E_{11}<E_{20}$ \\
\hline$s_{\Gamma_{11}}>s_{\Gamma_{20}}$ & $s_{\Gamma_{11}}>s_{\Gamma_{20}}$ & $s_{\Gamma_{11}}>s_{\Gamma_{20}}$ \\
\hline$s_{\rho_{11}}>s_{\rho_{20}}(<0.55)$ & $s_{\rho_{11}}>s_{\rho_{20}}$ & $s_{\rho_{11}}>s_{\rho_{20}}$ \\
$s_{\rho_{11}}<s_{\rho_{20}}(>0.55)$ & & \\
\hline$I_{x_{11}}<I_{x_{20}}$ & $I_{x_{11}}<I_{x_{20}}$ & $I_{x_{11}}<I_{x_{20}}(<1.045)$ \\
& & $I_{x_{11}}>I_{x_{20}}(>1.045)$ \\
\hline$s_{\Pi_{11}}>s_{\Pi_{20}}$ & $s_{\Pi_{11}}>s_{\Pi_{20}}$ & $s_{\Pi_{11}}>s_{\Pi_{20}}$ \\
\hline$s_{\pi_{11}}>s_{\pi_{20}}$ & $s_{\pi_{11}}>s_{\pi_{20}}$ & $s_{\pi_{11}}>s_{\pi_{20}}(<0.50)$ \\
& & $s_{\pi_{11}}<s_{\pi_{20}}(>0.50)$ \\
\hline$I_{p_{11}}<I_{p_{20}}(<1.55)$ & $I_{p_{11}}<I_{p_{20}}$ & $I_{p_{11}}<I_{p_{20}}$ \\
$I_{p_{11}}>I_{p_{20}}(>1.55)$ & & $s_{T_{11}}>s_{T_{20}}$ \\
\hline$s_{T_{11}}>s_{T_{20}}$ & $s_{T_{11}}>s_{T_{20}}$ & $s_{t_{11}}>s_{t_{20}}$ \\
\hline$s_{t_{11}}>s_{t_{20}}$ & $s_{t_{11}}>s_{t_{20}}$ & $I_{t_{11}}<I_{t_{20}}(<1.3)$ \\
\hline$I_{t_{11}}<I_{t_{20}}(<3.3)$ & $I_{t_{11}}<I_{t_{20}}$ \\
$I_{t_{11}}>I_{t_{20}(>3.3)}$ & & \\
\hline
\end{tabular}

Tabla 6.6: Comparación entre los estados $(1,1)$ y $(2,0)$. Los valores de $1 / \omega$ para los puntos de cruce se indican entre paréntesis y son aproximados. 


\begin{tabular}{|ccc|}
\hline $\begin{array}{c}(1,1),(0,2) \\
\text { Atractivo }\end{array}$ & No interactuante & Repulsivo \\
\hline$E_{11}<E_{02}$ & $E_{11}=E_{02}$ & $E_{11}>E_{02}$ \\
\hline$s_{\Gamma_{11}}>s_{\Gamma_{02}}$ & $s_{\Gamma_{11}}>s_{\Gamma_{02}}$ & $s_{\Gamma_{11}}>s_{\Gamma_{02}}$ \\
\hline$s_{\rho_{11}}>s_{\rho_{02}}$ & $s_{\rho_{11}}>s_{\rho_{02}}$ & $s_{\rho_{11}}>s_{\rho_{02}}(<0.51)$ \\
& & $s_{\rho_{11}}<s_{\rho_{02}}(>0.51)$ \\
\hline$I_{x_{11}}<I_{x_{02}}(<1.55)$ & $I_{x_{11}}<I_{x_{02}}$ & $I_{x_{11}}<I_{x_{02}}$ \\
$I_{x_{11}}>I_{x_{02}}(>1.55)$ & & \\
\hline$s_{\Pi_{11}}>s_{\Pi_{02}}$ & $s_{\Pi_{11}}>s_{\Pi_{02}}$ & $s_{\Pi_{11}}>s_{\Pi_{02}}$ \\
\hline$s_{\pi_{11}}>s_{\pi_{02}}(<0.55)$ & $s_{\pi_{11}}>s_{\pi_{02}}$ & $s_{\pi_{11}}>s_{\pi_{02}}$ \\
$s_{\pi_{11}}<s_{\pi_{02}}(>0.55)$ & & \\
\hline$I_{p_{11}}<I_{p_{02}}$ & $I_{p_{11}}<I_{p_{02}}$ & $I_{p_{11}}<I_{p_{02}}(<1.045)$ \\
& & $I_{p_{11}}>I_{p_{02}}(>1.045)$ \\
\hline$s_{T_{11}}>s_{T_{02}}$ & $s_{T_{11}}>s_{T_{02}}$ & $s_{T_{11}}>s_{T_{02}}$ \\
\hline$s_{t_{11}}>s_{t_{02}}$ & $s_{t_{11}}>s_{t_{02}}$ & $s_{t_{11}}>s_{t_{02}}$ \\
\hline$I_{t_{11}}<I_{t_{02}}(<3.3)$ & $I_{t_{11}}<I_{t_{02}}$ & $I_{t_{11}}<I_{t_{02}}(<1.3)$ \\
$I_{t_{11}}>I_{t_{02}}(>3.3)$ & & $I_{t_{11}}>I_{t_{02}}(>1.3)$ \\
\hline
\end{tabular}

Tabla 6.7: Comparación entre los estados $(1,1)$ y $(0,2)$. Los valores de $1 / \omega$ para los puntos de cruce se indican entre paréntesis y son aproximados. 


\begin{tabular}{|ccc|}
\hline $\begin{array}{c}(0,3),(1,2) \\
\text { Atractivo }\end{array}$ & No interactuante & Repulsivo \\
\hline$E_{03}>E_{12}$ & $E_{03}=E_{12}$ & $E_{03}<E_{12}$ \\
\hline$s_{\Gamma_{03}}<s_{\Gamma_{12}}$ & $s_{\Gamma_{03}}<s_{\Gamma_{12}}$ & $s_{\Gamma_{03}}<s_{\Gamma_{12}}$ \\
\hline$s_{\rho_{03}}<s_{\rho_{12}}$ & $s_{\rho_{03}}<s_{\rho_{12}}$ & $s_{\rho_{03}}<s_{\rho_{12}}(<1.25)$ \\
& & $s_{\rho_{03}}>s_{\rho_{12}}(>1.25)$ \\
\hline$I_{x_{03}}>I_{x_{12}}(<2.12)$ & $I_{x_{03}}>I_{x_{12}}$ & $I_{x_{03}}>I_{x_{12}}$ \\
$I_{x_{03}}<I_{x_{12}}(>2.12)$ & & $s_{\Pi_{03}}<s_{\Pi_{12}}$ \\
\hline$s_{\Pi_{03}}<s_{\Pi_{12}}$ & $s_{\Pi_{03}}<s_{\Pi_{12}}$ & $s_{\pi_{03}}<s_{\pi_{12}}$ \\
\hline$s_{\pi_{03}}<s_{\pi_{12}}(<2.55)$ & $s_{\pi_{03}}<s_{\pi_{12}}$ & \\
$s_{\pi_{03}}>s_{\pi_{12}}(>2.55)$ & & $I_{p_{03}}>I_{p_{12}}(<1.176)$ \\
\hline$I_{p_{03}}>I_{p_{12}}$ & $I_{p_{03}}>I_{p_{12}}$ & $I_{p_{03}}<I_{p_{12}}(>1.176)$ \\
\hline$s_{T_{03}}<s_{T_{12}}$ & & $s_{T_{03}}<s_{T_{12}}$ \\
\hline$s_{t_{03}}<s_{t_{12}}$ & $s_{T_{03}}<s_{T_{12}}(>1.36)$ \\
\hline$I_{t_{03}}>I_{t_{12}}(<4.45)$ & $s_{t_{03}}<s_{t_{12}}$ & $s_{t_{03}}<s_{t_{12}}$ \\
$I_{t_{03}}<I_{t_{12}}(>4.45)$ & & $I_{t_{03}}>I_{t_{12}}(<1.36)$ \\
\hline
\end{tabular}

Tabla 6.8: Comparación entre los estados $(0,3)$ y $(1,2)$. Los valores de $1 / \omega$ para los puntos de cruce se indican entre paréntesis y son aproximados. 


\begin{tabular}{|c|c|c|}
\hline$(3,1),(2,2)$ & & \\
\hline Atractivo & No interactuante & Repulsivo \\
\hline$E_{31}<E_{22}$ & $E_{31}=E_{22}$ & $E_{31}>E_{22}$ \\
\hline$s_{\Gamma_{31}}<s_{\Gamma_{22}}$ & $s_{\Gamma_{31}}<s_{\Gamma_{22}}$ & $s_{\Gamma_{31}}<s_{\Gamma_{22}}$ \\
\hline$s_{\rho_{31}}>s_{\rho_{22}}$ & $s_{\rho_{31}}>s_{\rho_{22}}$ & $\begin{array}{l}s_{\rho_{31}}>s_{\rho_{22}}(<0.63) \\
s_{\rho_{31}}<s_{\rho_{22}}(>0.63)\end{array}$ \\
\hline$I_{x_{31}}>I_{x_{22}}$ & $I_{x_{31}}>I_{x_{22}}$ & $\begin{array}{l}I_{x_{31}}>I_{x_{22}}(<0.96) \\
I_{x_{31}}<I_{x_{22}}(>0.96)\end{array}$ \\
\hline$s_{\Pi_{31}}<s_{\Pi_{22}}$ & $s_{\Pi_{31}}<s_{\Pi_{22}}$ & $s_{\Pi_{31}}<s_{\Pi_{22}}$ \\
\hline $\begin{array}{l}s_{\pi_{31}}>s_{\pi_{22}}(<0.70) \\
s_{\pi_{31}}<s_{\pi_{22}}(>0.70)\end{array}$ & $s_{\pi_{31}}>s_{\pi_{22}}$ & $s_{\pi_{31}}>s_{\pi_{22}}$ \\
\hline $\begin{array}{l}I_{p_{31}}>I_{p_{22}}(<1.3) \\
I_{p_{31}}<I_{p_{22}}(>1.3)\end{array}$ & $I_{p_{31}}>I_{p_{22}}$ & $I_{p_{31}}>I_{p_{22}}$ \\
\hline$s_{T_{31}}<s_{T_{22}}$ & $s_{T_{31}}<s_{T_{22}}$ & $s_{T_{31}}<s_{T_{22}}$ \\
\hline $\begin{array}{l}s_{t_{31}}>s_{t_{22}}(<1.3) \\
s_{t_{31}}<s_{t_{22}}(>1.3)\end{array}$ & $s_{t_{31}}>s_{t_{22}}$ & $\begin{array}{l}s_{t_{31}}>s_{t_{22}}(<0.96) \\
s_{t_{31}}<s_{t_{22}}(>0.96)\end{array}$ \\
\hline $\begin{array}{l}I_{t_{31}}>I_{t_{22}}(<2.36) \\
I_{t_{31}}<I_{t_{22}}(>2.36)\end{array}$ & $I_{t_{31}}>I_{t_{22}}$ & $\begin{array}{l}I_{t_{31}}>I_{t_{22}}(<1.21) \\
I_{t_{31}}<I_{t_{22}}(>1.21)\end{array}$ \\
\hline
\end{tabular}

Tabla 6.9: Comparación entre los estados $(3,1)$ y $(2,2)$. Los valores de $1 / \omega$ para los puntos de cruce se indican entre paréntesis y son aproximados. 


\begin{tabular}{|c|c|c|}
\hline$(1,3),(2,2)$ & & \\
\hline Atractivo & No interactuante & Repulsivo \\
\hline$E_{13}>E_{22}$ & $E_{13}=E_{22}$ & $E_{13}<E_{22}$ \\
\hline$s_{\Gamma_{13}}<s_{\Gamma_{22}}$ & $s_{\Gamma_{13}}<s_{\Gamma_{22}}$ & $s_{\Gamma_{13}}<s_{\Gamma_{22}}$ \\
\hline $\begin{array}{l}s_{\rho_{13}}>s_{\rho_{22}}(<0.70) \\
s_{\rho_{13}}<s_{\rho_{22}}(>0.70)\end{array}$ & $s_{\rho_{13}}>s_{\rho_{22}}$ & $s_{\rho_{13}}>s_{\rho_{22}}$ \\
\hline $\begin{array}{l}I_{x_{13}}>I_{x_{22}}(<1.3) \\
I_{x_{13}}<I_{x_{22}}(>1.3)\end{array}$ & $I_{x_{13}}>I_{x_{22}}$ & $I_{x_{13}}>I_{x_{22}}$ \\
\hline$s_{\Pi_{13}}<s_{\Pi_{22}}$ & $s_{\Pi_{13}}<s_{\Pi_{22}}$ & $s_{\Pi_{13}}<s_{\Pi_{22}}$ \\
\hline$s_{\pi_{13}}>s_{\pi_{22}}$ & $s_{\pi_{13}}>s_{\pi_{22}}$ & $\begin{array}{l}s_{\pi_{13}}>s_{\pi_{22}}(<0.63) \\
s_{\pi_{13}}<s_{\pi_{22}}(>0.63)\end{array}$ \\
\hline$I_{p_{13}}>I_{p_{22}}$ & $I_{p_{13}}>I_{p_{22}}$ & $\begin{array}{l}I_{p_{13}}>I_{p_{22}}(<0.96) \\
I_{p_{13}}<I_{p_{22}}(>0.96)\end{array}$ \\
\hline$s_{T_{13}}<s_{T_{22}}$ & $s_{T_{13}}<s_{T_{22}}$ & $s_{T_{13}}<s_{T_{22}}$ \\
\hline $\begin{array}{l}s_{t_{13}}>s_{t_{22}}(<1.3) \\
s_{t_{13}}<s_{t_{22}}(>1.3)\end{array}$ & $s_{t_{13}}>s_{t_{22}}$ & $\begin{array}{l}s_{t_{13}}>s_{t_{22}}(<0.96) \\
s_{t_{13}}<s_{t_{22}}(>0.96)\end{array}$ \\
\hline $\begin{array}{l}I_{t_{13}}>I_{t_{22}}(<2.36) \\
I_{t_{13}}<I_{t_{22}}(>2.36)\end{array}$ & $I_{t_{13}}>I_{t_{22}}$ & $\begin{array}{l}I_{t_{13}}>I_{t_{22}}(<1.21) \\
I_{t_{13}}<I_{t_{22}}(>1.21)\end{array}$ \\
\hline
\end{tabular}

Tabla 6.10: Comparación entre los estados $(1,3)$ y $(2,2)$. Los valores de $1 / \omega$ para los puntos de cruce se indican entre paréntesis y son aproximados.

\subsubsection{Localización en la densidad de pares}

Vamos a analizar ahora la localización en la densidad de pares de los estados $[(2,1),(3,0)]$. En la Tabla (6.5) se oberva que en el límite no degenerado $s_{\Gamma_{21}}>s_{\Gamma_{30}}$ y $s_{\Pi_{21}}>s_{\Pi_{30}}$. Este orden relativo no se afecta cuando se enciende el potencial, sin importar que se trate del caso atractivo o repulsivo. De hecho las curvas son equidistantes en todo el intervalo de la magnitud del potencial y el signo entre las entropías es igual en ambos 
espacios. Esto es, el estado más deslocalizado en el espacio de posición es también el más deslocalizado en espacio de momento. Por inspección en las Tablas (6.6), (6.7), (6.8), (6.9) y (6.10) puede notarse que esto último es una característica general.

Las Tablas pueden clasificarse según el ordenamiento de la localización en la densidad de pares de los estados:

- $[(2,1),(3,0)],[(1,1),(2,0)] \mathrm{y}[(1,1),(0,2)]$. Tablas $(6.5),(6.6)$ y (6.7). El estado antisimétrico está más deslocalizado que el simétrico.

- $[(0,3),(1,2)],[(3,1),(2,2)] \mathrm{y}[(1,3),(2,2)]$. Tablas $(6.8),(6.9)$ y $(6.10)$. El estado simétrico está más deslocalizado que el antisimétrico.

La magnitud de la diferencia $\left|n_{r}-n_{R}\right|$ puede relacionarse con el ordenamiento en la localización. Como se desprende de esta clasificación el estado que tiene la menor diferencia es el más deslocalizado en los espacios de posición y momento independientemente del potencial (atractivo o repulsivo).

\subsubsection{Localización en la densidad reducida de una variable}

Los números cuánticos también juegan un papel en los valores de las entropías de la densidad reducida en el límite no interactuante, y por lo tanto determinan el orden relativo. La Tabla (6.5) muestra que en este caso tenemos $s_{\rho_{21}}>s_{\rho_{30}}$ y $s_{\pi_{21}}>s_{\pi_{30}}$.

Analizaremos la existencia de cruces entre las entropías, para ello tomemos como ejemplo las entropías en el espacio de posición. En el límite no interactuante, $E_{21}=E_{30}$ y $s_{\rho_{21}}>s_{\rho_{30}}$. Cuando encendemos el potencial repulsivo provocamos que $E_{21}<E_{30}$. Usando los argumentos del teorema virial que vertimos la sección anterior $\left(\bar{V}_{21}<\bar{V}_{30}\right)$, podemos entender por qué el ordenamiento que tienen las entropías en el límite no interactuante se mantiene en presencia de este potencial repulsivo.

Por otra parte, al encender el potencial atractivo logramos que $E_{21}>E_{30}$, entonces $\left(\bar{V}_{21}>\bar{V}_{30}\right)$ y por tanto esperamos que $s_{\rho_{21}}<s_{\rho_{30}}$. La presencia de un potencial atractivo debería inducir que se invierta el orden relativo de las entropías conforme nos 
alejamos del límite no interactuante. Y así es, el valor de $1 / \omega$ del punto en que ocurre el cambio de orden de estas entropías se muestra en la Tabla (6.5).

Argumentos similares pueden utilizarse para las entropías en el espacio de momento. En el régimen repulsivo, $E_{21}<E_{30}$, y entonces $\bar{T}_{21}<\bar{T}_{30}$. Esperaríamos que la densidad de momento esté más dispersa o deslocalizada en el estado $(3,0)$, y por tanto que $s_{\pi_{21}}<s_{\pi_{30}}$. Este ordenamiento es opuesto al que se observa en el límite no interactuante. Si prendemos el potencial repulsivo debería resultar una inversión en el orden de las entropías, y efectivamente así es, el valor de $1 / \omega$ en que este reordenamiento ocurre se muestra en la Tabla (6.5). En cambio, si encendemos el potencial atractivo tenemos que $\bar{T}_{21}>\bar{T}_{30}$ y por lo tanto esperamos que $s_{\pi_{21}}>s_{\pi_{30}}$, este es el mismo orden que en el límite no interactuante y por ello no se observa un cruce. El comportamiento de las entropías alrededor del punto en que cruzan se muestra en la Fig. (6.6).
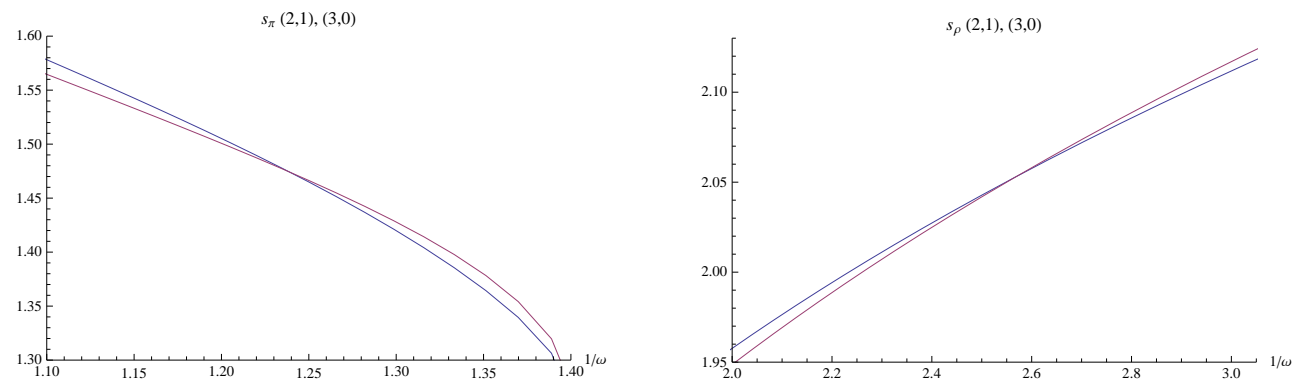

Figura 6.6: Detalle de los cruces que ocurren en los estados $(2,1)$ [azul] y $(3,0)$ [rojo]. $s_{\pi_{21}}$ y $s_{\pi_{30}}$ con potencial repulsivo (izquierda). $s_{\rho_{21}}$ y $s_{\rho_{30}}$ con potencial atractivo (derecha).

Exactamente los mismos argumentos energéticos pueden utilizarse para explicar los cruces que ocurren en todas las Tablas. Dado un régimen de potencial siempre ocurre un cruce en alguno de los espacios y en dos casos, Tablas (6.9) y (6.10), para ambos regímenes de potencial ocurre un cruce en $s_{t}$, una medida de la localización en un espacio fase separable.

El resultado principal de esta sección es que el potencial entre partículas puede usarse para controlar la localización de la densidad reducida de un estado relativo a otro con diferente simetría. Esto ilustra la influencia mutua entre la simetría y el 
potencial.

\subsubsection{Correlación estadística}

Es razonable esperar un punto de cruce en las medidas de correlación porque hay cruces entre las entropías reducidas de una partícula. Para el par de estados en que nos hemos concentrado estos puntos se reportan en la Tabla (6.5) y las informaciones mutuas se grafican en la Fig. (6.7). La interpretación de este comportamiento es que la magnitud de la correlación en un estado relativo al otro de diferente simetría puede controlarse por medio del potencial entre partículas.
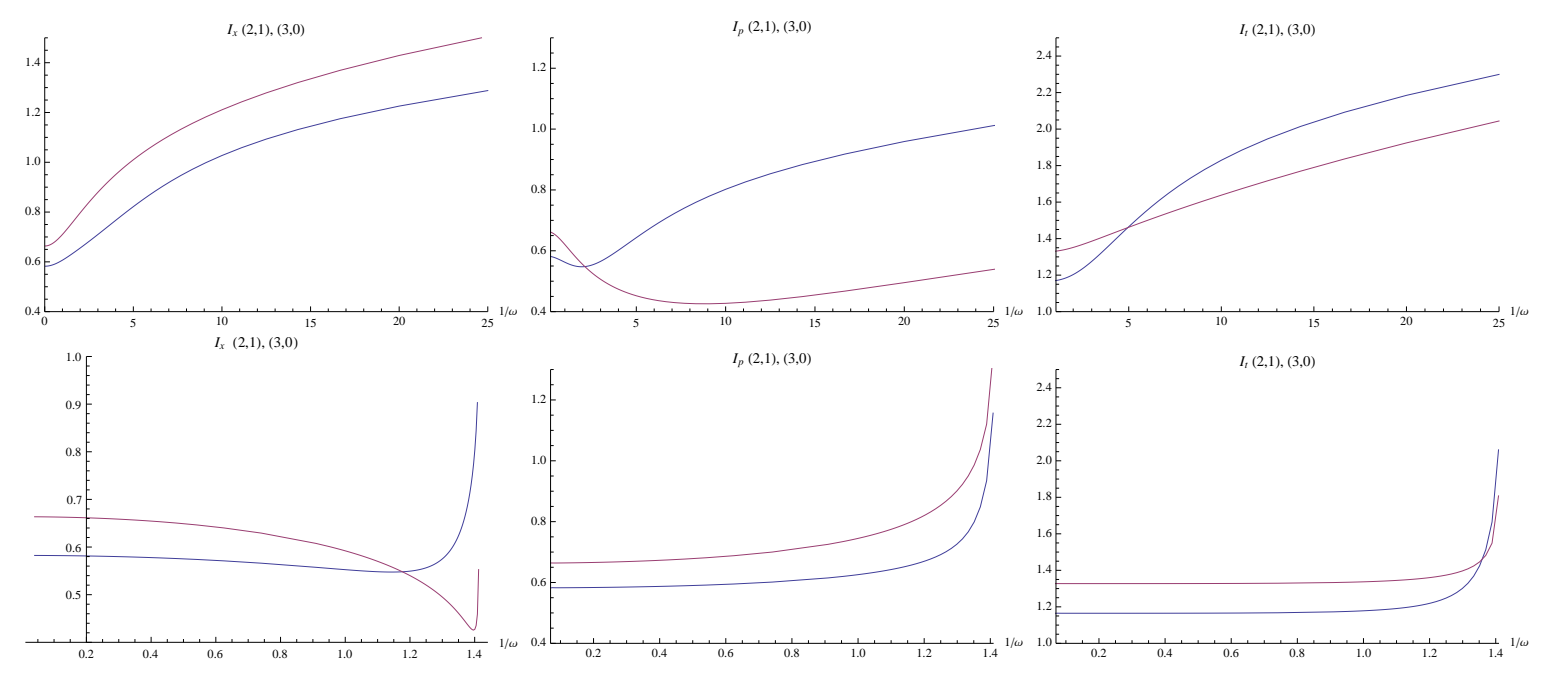

Figura 6.7: Información mutua para los estados $[(2,1)[$ azul],(3,0)[rojo]] con potencial atractivo (arriba) y potencial repulsivo (abajo).

Como se ha señalado, en todos los casos estudiados existe un punto de cruce en alguno de los espacios (dado un potencial cruza $I_{x}$ o bien cruza $I_{p}$ ). En todos los casos ocurre un cruce en la correlación total entre partículas, $I_{t}$. Esto demuestra la importancia del potencial para gobernar el ordenamiento de la correlación entre estados, resaltamos que este control puede ejercerse en estados con diferente simetría.

En el caso discutido,Tabla (6.5), el cruce ocurre en el espacio de posición para el potencial atractivo en tanto que otro cruce ocurre en el espacio de momento para el 
repulsivo. Esta es una característica general del modelo: Si ocurre un cruce en uno de los espacios bajo un régimen de potencial existirá otro cruce en el otro espacio bajo el otro régimen de potencial.

\subsection{Otras características en la simetría del modelo}

Para ser exhaustivos en el análisis de la interrelación entre la simetría y el potencial en este modelo examinaremos brevemente otros pares de estados con características diferentes a los que hemos estudiado en las secciones anteriores. Los pares de estados que examinamos antes comparten la característica de poseer difierente simetría, por lo que el hueco de Fermi es siempre una variable para el análisis. Ahora dirigimos nuestra atención hacia pares de estados cuyas funciones de onda son ambas simétricas o antisimétricas.

\begin{tabular}{|ccc|}
\hline $\begin{array}{c}(2,0),(0,2) \\
\text { Atractivo }\end{array}$ & No interactuante & Repulsivo \\
\hline$E_{20}<E_{02}$ & $E_{20}=E_{02}$ & $E_{20}>E_{02}$ \\
\hline$s_{\Gamma_{20}}=s_{\Gamma_{02}}$ & $s_{\Gamma_{20}}=s_{\Gamma_{02}}$ & $s_{\Gamma_{20}}=s_{\Gamma_{02}}$ \\
\hline$s_{\rho_{20}}>s_{\rho_{02}}$ & $s_{\rho_{20}}=s_{\rho_{02}}$ & $s_{\rho_{20}}<s_{\rho_{02}}$ \\
\hline$I_{x_{20}}>I_{x_{02}}$ & $I_{x_{20}}=I_{x_{02}}$ & $I_{x_{20}}<I_{x_{02}}$ \\
\hline$s_{\Pi_{20}}=s_{\Pi_{02}}$ & $s_{\Pi_{20}}=s_{\Pi_{02}}$ & $s_{\Pi_{20}}=s_{\Pi_{02}}$ \\
\hline$s_{\pi_{20}}<s_{\pi_{02}}$ & $s_{\pi_{20}}=s_{\pi_{02}}$ & $s_{\pi_{20}}>s_{\pi_{02}}$ \\
\hline$I_{p_{20}}<I_{p_{02}}$ & $I_{p_{20}}=I_{p_{02}}$ & $I_{p_{20}}>I_{p_{02}}$ \\
\hline$s_{T_{20}}=s_{T_{02}}$ & $s_{T_{20}}=s_{T_{02}}$ & $s_{T_{20}}=s_{T_{02}}$ \\
\hline$s_{t_{20}}=s_{t_{02}}$ & $s_{t_{20}}=s_{t_{02}}$ & $s_{t_{20}}=s_{t_{02}}$ \\
\hline$I_{t_{20}}=I_{t_{02}}$ & $I_{t_{20}}=I_{t_{02}}$ & $I_{t_{20}}=I_{t_{02}}$ \\
\hline
\end{tabular}

Tabla 6.11: Comparación entre los estados $(2,0)$ y $(0,2)$.

Consideremos por ejemplo el par de estados $[(2,0),(0,2)]$, Tabla $(6.11)$, donde no 
hay huecos de simetría, ni el de Fermi ni el de contrapeso y ambas funciones de onda son simétricas. Una característica de estos estados es que la localización de las densidades de pares es la misma (ambas entropías tienen el mismo valor) independientemente de la magnitud del potencial, que es una situación similar a la que se presenta entre los estados $[(2,1),(1,2)]$ cuyas relaciones se muestran en la Tabla (6.1). En el límite no interactuante tenemos que $\rho_{20}(x)=\rho_{02}(x)$, las entropías de Shannon de las densidades reducidas correspondientes tienen el mismo valor. La influencia del potencial sobre las entropías conforme nos alejamos del límite no interactuante es la misma que la que se discutió para el par de estados $[(2,1),(1,2)]$.

\begin{tabular}{|ccc|}
\hline $\begin{array}{c}(1,3),(3,1) \\
\text { Atractivo }\end{array}$ & No interactuante & Repulsivo \\
\hline$E_{13}>E_{31}$ & $E_{13}=E_{31}$ & $E_{13}<E_{31}$ \\
\hline$s_{\Gamma_{13}}=s_{\Gamma_{31}}$ & $s_{\Gamma_{13}}=s_{\Gamma_{31}}$ & $s_{\Gamma_{13}}=s_{\Gamma_{31}}$ \\
\hline$s_{\rho_{13}}<s_{\rho_{31}}$ & $s_{\rho_{13}}=s_{\rho_{31}}$ & $s_{\rho_{13}}>s_{\rho_{31}}$ \\
\hline$I_{x_{13}}<I_{x_{31}}$ & $I_{x_{13}}=I_{x_{31}}$ & $I_{x_{13}}>I_{x_{31}}$ \\
\hline$s_{\Pi_{13}}=s_{\Pi_{31}}$ & $s_{\Pi_{13}}=s_{\Pi_{31}}$ & $s_{\Pi_{13}}=s_{\Pi_{31}}$ \\
\hline$s_{\pi_{13}}>s_{\pi_{31}}$ & $s_{\pi_{13}}=s_{\pi_{31}}$ & $s_{\pi_{13}}<s_{\pi_{31}}$ \\
\hline$I_{p_{13}}>I_{p_{31}}$ & $I_{p_{13}}=I_{p_{31}}$ & $I_{p_{13}}<I_{p_{31}}$ \\
\hline$s_{T_{13}}=s_{T_{31}}$ & $s_{T_{13}}=s_{T_{31}}$ & $s_{T_{13}}=s_{T_{31}}$ \\
\hline$s_{t_{13}}=s_{t_{31}}$ & $s_{t_{13}}=s_{t_{31}}$ & $s_{t_{13}}=s_{t_{31}}$ \\
\hline$I_{t_{13}}=I_{t_{31}}$ & $I_{t_{13}}=I_{t_{31}}$ & $I_{t_{13}}=I_{t_{31}}$ \\
\hline
\end{tabular}

Tabla 6.12: Comparación entre los estados $(1,3)$ y $(3,1)$.

Ahora discutiremos el par de estados $[(1,3),(3,1)]$ que tienen funciones antisimétricas. Una diferencia con el caso discutido en los párrafos previos es que los dos huecos están presentes en ambos estados. Este par comparte, con algunos analizados en secciones previas, la característica de que los números cuánticos sólo han sido intercam- 
biados. Este par conduce a resultados del mismo tipo que los discutidos para el caso $[(2,1),(1,2)]$. La síntesis de los datos puede observarse en la Tabla (6.12).

Ahora veremos el par $[(1,2),(3,0)]$. Ambos estados son simétricos (ho hay hueco de Fermi) pero los dos tienen el hueco de contrapeso debido a que $n_{R}$ es impar. En el límite no interactuante diferentes orbitales se encuentran ocupados, como es el caso de algunos pares de estados discutidos en secciones previas de este capítulo. Las relaciones entre las cantidades se presentan en la Tabla (6.13). Puede utilizarse el mismo tipo de análisis y consideraciones energéticas que sirvieron para estudiar los estados $[(0,3),(1,2)]$ para explicar el comportamiento y los cruces de las medidas de localización y correlación. Las entropías de pares del estado $(1,2)$ son siempre mayores que las entropías del estado $(3,0)$. En ambos regímenes de potencial ocurre un cruce en la correlación total entre partículas, $I_{t}$. Para el potencial atractivo ocurren además cruces en $s_{\rho}$ e $I_{p}$, en tanto que para el potencial repulsivo ocurren los cruces en $s_{\pi}$ e $I_{x}$.

Finalmente, en la Tabla (6.14) se muestran los resultados para dos estados antisimétricos, $[(0,3),(2,1)]$, que sólo contienen el hueco de Fermi. Sus características pueden discutirse siguiendo los mismos argumentos que se detallaron para analizar los estados $[(0,3),(1,2)]$ anteriormente. En este caso las entropías de pares del estado $(2,1)$ son siempre mayores que las entropías del estado $(0,3)$. También en ambos regímenes de potencial ocurre un cruce en la correlación total entre partículas, $I_{t}$. Para el potencial atractivo ocurren además cruces en $s_{\pi}$ e $I_{x}$, en tanto que para el potencial repulsivo ocurren los cruces en $s_{\rho}$ e $I_{p}$. 


\begin{tabular}{|ccc|}
\hline $\begin{array}{c}(1,2),(3,0) \\
\text { Atractivo }\end{array}$ & No interactuante & Repulsivo \\
\hline$E_{12}>E_{30}$ & $E_{12}=E_{30}$ & $E_{12}<E_{30}$ \\
\hline$s_{\Gamma_{12}}>s_{\Gamma_{30}}$ & $s_{\Gamma_{12}}>s_{\Gamma_{30}}$ & $s_{\Gamma_{12}}>s_{\Gamma_{30}}$ \\
\hline$s_{\rho_{12}}>s_{\rho_{30}}(<1.10)$ & $s_{\rho_{12}}>s_{\rho_{30}}$ & $s_{\rho_{12}}>s_{\rho_{30}}$ \\
$s_{\rho_{12}}<s_{\rho_{30}}(>1.10)$ & & \\
\hline$I_{x_{12}}<I_{x_{30}}$ & $I_{x_{12}}<I_{x_{30}}$ & $I_{x_{12}}<I_{x_{30}}(<0.87)$ \\
& & $I_{x_{12}}>I_{x_{30}}(>0.87)$ \\
\hline$s_{\Pi_{12}}>s_{\Pi_{30}}$ & $s_{\Pi_{12}}>s_{\Pi_{30}}$ & $s_{\Pi_{12}}>s_{\Pi_{30}}$ \\
\hline$s_{\pi_{12}}>s_{\pi_{30}}$ & $s_{\pi_{12}}>s_{\pi_{30}}$ & $s_{\pi_{12}}>s_{\pi_{30}}(<0.87)$ \\
& & $s_{\pi_{12}}<s_{\pi_{30}}(>0.87)$ \\
\hline$I_{p_{12}}<I_{p_{30}}(<1.12)$ & $I_{p_{12}}<I_{p_{30}}$ & $I_{p_{12}}<I_{p_{30}}$ \\
$I_{p_{12}}>I_{p_{30}}(>1.12)$ & & $s_{t_{12}}>I_{t_{30}}(>1.36)$ \\
\hline$s_{T_{12}}>s_{T_{30}}$ & $s_{T_{12}}>s_{T_{30}}$ \\
\hline$s_{t_{12}}>s_{t_{30}}$ & $s_{t_{12}}>s_{t_{30}}$ & $s_{t_{12}}>s_{t_{30}}$ \\
\hline$I_{t_{12}}<I_{t_{30}}(<4.95)$ & $I_{t_{12}}<I_{t_{30}}$ & $I_{t_{12}}<I_{t_{30}}(<1.36)$ \\
$I_{t_{12}}>I_{t_{30}(>4.95)}$ & & \\
\hline
\end{tabular}

Tabla 6.13: Comparación entre los estados $(1,2)$ y $(3,0)$. Los valores de $1 / \omega$ para los puntos de cruce se indican entre paréntesis y son aproximados. 


\begin{tabular}{|ccc|}
\hline $\begin{array}{c}(0,3),(2,1) \\
\text { Atractivo }\end{array}$ & No interactuante & Repulsivo \\
\hline$E_{03}>E_{21}$ & $E_{03}=E_{21}$ & $E_{03}<E_{21}$ \\
\hline$s_{\Gamma_{03}}<s_{\Gamma_{21}}$ & $s_{\Gamma_{03}}<s_{\Gamma_{21}}$ & $s_{\Gamma_{03}}<s_{\Gamma_{21}}$ \\
\hline$s_{\rho_{03}}<s_{\rho_{21}}$ & $s_{\rho_{03}}<s_{\rho_{21}}$ & $s_{\rho_{03}}<s_{\rho_{21}}(<0.87)$ \\
& & $s_{\rho_{03}}>s_{\rho_{21}}(>0.87)$ \\
\hline$I_{x_{03}}>I_{x_{21}}(<1.12)$ & $I_{x_{03}}>I_{x_{21}}$ & $I_{x_{03}}>I_{x_{21}}$ \\
$I_{x_{03}}<I_{x_{21}}(>1.12)$ & & $s_{\Pi_{03}}<s_{\Pi_{21}}$ \\
\hline$s_{\Pi_{03}}<s_{\Pi_{21}}$ & $s_{\Pi_{03}}<s_{\Pi_{21}}$ & $s_{\pi_{03}}<s_{\pi_{21}}$ \\
\hline$s_{\pi_{03}}<s_{\pi_{21}}(<1.1)$ & $s_{\pi_{03}}<s_{\pi_{21}}$ & \\
$s_{\pi_{03}}>s_{\pi_{21}}(>1.1)$ & & $I_{p_{03}}>I_{p_{21}}(<0.87)$ \\
\hline$I_{p_{03}}>I_{p_{21}}$ & $I_{p_{03}}>I_{p_{21}}$ & $I_{p_{03}}<I_{p_{21}}(>0.87)$ \\
\hline$s_{T_{03}}<s_{T_{21}}$ & $s_{T_{03}}<s_{T_{21}}$ & $s_{T_{21}}<s_{t_{21}}$ \\
\hline$s_{t_{03}}<s_{t_{21}}$ & $s_{t_{03}}<s_{t_{21}}$ & $I_{t_{03}}>I_{t_{21}}(<1.36)$ \\
\hline$I_{t_{03}}>I_{t_{21}(<4.95)}(>1.36)$ \\
$I_{t_{03}}<I_{t_{21}(>4.95)}$ & $I_{t_{03}}>I_{t_{21}}$ \\
\hline
\end{tabular}

Tabla 6.14: Comparación entre los estados $(0,3)$ y $(2,1)$. Los valores de $1 / \omega$ para los puntos de cruce se indican entre paréntesis y son aproximados.

\subsection{Conclusiones}

Las entropías de Shannon de la densidad de pares no distinguen entre la presencia de un hueco de Fermi (función de onda antisimétrica) y de un hueco de contrapeso (función de onda simétrica) en el modelo del átomo de Moshinsky. Estados que ocupan los mismos orbitales en el límite no interactuante pero que difieren en la simetría de la función de onda tienen la misma entropía de pares en el espacio de posición, esto ocurre también 
en el espacio de momento y es independiente de la naturaleza y de la magnitud del potencial de interacción.

Por otro lado, las entropías de las densidades reducidas de una partícula en estos estados distinguen entre las funciones simétrica y antisimétrica. Para sistemas no interactuantes, las densidades reducidas y, por lo tanto, las entropías de estos estados con diferente simetría son iguales. El potencial entre partículas provoca que se diferencien.

Con un potencial atractivo entre partículas, la densidad reducida de una partícula en el espacio de posición está más deslocalizada comparada con la simétrica. En el espacio de momento la densidad correspondiente a la función de onda antisimétrica está más localizada que la simétrica. Esta situación es inversa con potenciales repulsivos. La densidad de la función antisimétrica está ahora más localizada en el espacio de posición y más deslocalizada en el espacio de momento comparada con el caso simétrico.

La correlación estadística, medida a través de la información mutua, depende del balance entre las medidas de localización en los niveles de una y dos partículas. En estos estados en que la entropía de pares es igual, la densidad reducida más deslocalizada implica mayor correlación. Esto conduce al resultado de que con un potencial atractivo las partículas que obedecen una función antisimétrica están más correlacionadas en el espacio de posición, en tanto que las partículas cuya función es simétrica están más correlacionadas en el espacio de momento. La situación es inversa con un potencial repulsivo. Las partículas de la función antisimétrica están ahora más correlacionadas en el espacio de momento y las partículas de la función simétrica están más correlacionadas en el espacio de posición.

La existencia de mínimos en las gráficas de las medidas de correlación como función de la magnitud del potencial entre partículas puede explicarse mediante las diferentes respuestas de las densidades de pares y reducida al potencial y al balance entre las medidas de localización en los niveles de una y dos partículas.

Comparamos funciones simétricas y antisimétricas que ocupan diferentes orbitales en el límite no interactuante, en estos casos el orden relativo de la localización/deslocalización en las densidades y de la correlación estadística entre partículas, pueden regularse var- 
iando la intensidad de la trampa armónica. Esto es, existen puntos en los que se cruzan las curvas respectivas en ambos espacios para potenciales atractivos y repulsivos. Estos resultados ilustran la mutua influencia entre la simetría de la función de onda y el potencial entre partículas y cómo ésta impacta en la localización de las funciones de distribución y en la correlación estadística entre partículas. 


\section{El átomo de Moshinsky en el espacio fase: Correlaciones entre posición y momento}

\subsection{Localización}

\subsubsection{Distribuciones de dos partículas}

En este capítulo analizaremos la localización en el espacio fase. Expresiones entrópicas formuladas en términos de distribuciones mecano-cuánticas en el espacio fase han sido exploradas en la literatura [114,61,62]. La entropía de Shannon de la función de Wigner se ha estudiado también en sistemas modelo de una partícula. Debido a la presencia del logaritmo tiene un componente real y uno imaginario en sistemas donde la función de Wigner tiene valores negativos [132]. La entropía de Shannon de la función de Wigner de dos partículas se define,

$$
s_{W}=\operatorname{Re}\left[s_{W}\right]+\operatorname{Im}\left[s_{W}\right]
$$

con

$$
\begin{aligned}
\operatorname{Re}\left[s_{W}\right] & =-\int_{+} d x_{1} d p_{1} d x_{2} d p_{2} W^{+}\left(x_{1}, p_{1}, x_{2}, p_{2}\right) \ln W^{+}\left(x_{1}, p_{1}, x_{2}, p_{2}\right) \\
& +\int_{-} d x_{1} d p_{1} d x_{2} d p_{2}\left|W^{-}\left(x_{1}, p_{1}, x_{2}, p_{2}\right)\right| \ln \left|W^{-}\left(x_{1}, p_{1}, x_{2}, p_{2}\right)\right|
\end{aligned}
$$




$$
\operatorname{Im}\left[s_{W}\right]=i \pi \int_{-} d x_{1} d p_{1} d x_{2} d p_{2}\left|W^{-}\left(x_{1}, p_{1}, x_{2}, p_{2}\right)\right| .
$$

Como en el caso de una partícula, las ecuaciones anteriores se deben a que la integración puede separarse en las regiones positivas $(+)$ y negativas (-) de la función de Wigner y se han tomado valores absolutos en las partes negativas. Si analizamos la parte real obervamos que la localización tiene contribuciones de ambas regiones y que éstas son de signo contrario y por tanto contribuyen de manera exactamente opuesta a la entropía. La parte imaginaria es proporcional al volumen de las regiones negativas.

En la Fig. (7.1) se muestra el comportamiento de $s_{W}$ como función del número cuántico de las coordenadas relativas, $n_{r}$. Se observa que los componentes real e imaginario aumentan, la interpretación de este comportamiento es que la función de Wigner de dos partículas se deslocaliza con la excitación. También verificamos numéricamente que para un estado $s_{W}$ no depende del régimen de potencial entre partículas (atractivo o repulsivo) ni de su magnitud, es decir, ambos componentes son constantes con $\kappa$.
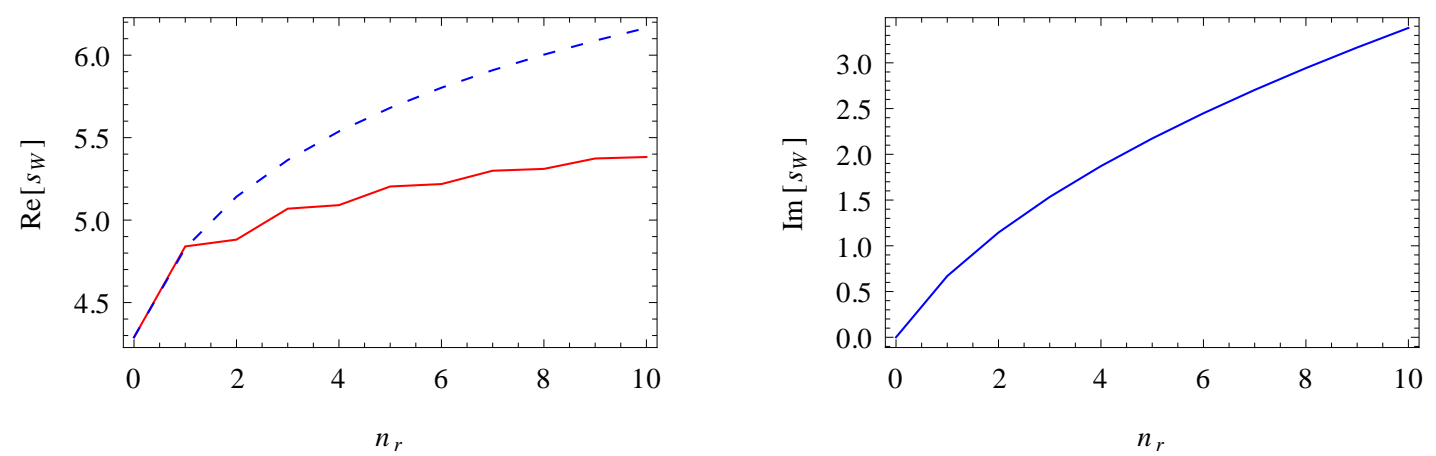

Figura 7.1: Entropía de Shannon de la función de Wigner de dos partículas, $s_{W}$, con la parte real (izquierda, rojo) y la parte imaginaria (derecha, azul), como funciones del número cuántico, $n_{r}$, para $n_{R}=0$. También se muestra la suma entrópica de dos partículas, $s_{T}$, (izquierda, azul, discontinua).

La entropía de Shannon de la función de Wigner de dos partículas puede considerarse como una generalización de la suma entrópica (relación de incertidumbre) en el 
nivel de dos partículas [106],

$$
\begin{aligned}
& s_{T}=s_{\Gamma}+s_{\Pi}=-\int d x_{1} d p_{1} d x_{2} d p_{2} \Gamma\left(x_{1}, x_{2}\right) \Pi\left(p_{1}, p_{2}\right) \ln \left[\Gamma\left(x_{1}, x_{2}\right) \Pi\left(p_{1}, p_{2}\right)\right] \\
& \geq 2(1+\ln \pi) .
\end{aligned}
$$

La suma entrópica, $s_{T}$, mide la localización en una distribución de dos partículas en un espacio fase separable mientras que $s_{W}$ es una medida de la localización en la función de Wigner, una distribución que en general no es separable. $s_{T}$ no depende del potencial y es similar a $s_{W}$ en este aspecto. Ambas aumentan con $n_{r}$ como puede observarse en la Fig. (7.1). Esto es, las distribuciones asociadas se deslocalizan con la excitación.

\subsubsection{Distribuciones reducidas de una partícula}

Analizaremos ahora la localización en las distribuciones reducidas en espacio fase separable $[\rho(x) \pi(p)]$ y no separable $[w(x, p)]$. Estas distribuciones se obtienen por integración de las de dos partículas sobre $d x_{2}$ y $d p_{2}$.

La función de Wigner reducida de una partícula es positivo-definida, esto debido a que la acción de "vestir" una partícula en la otra remueve las regiones negativas de la función de Wigner de dos partículas asociada con este sistema y, por lo tanto, $s_{w}$ es real. Como un ejemplo, mostramos en la Fig. (7.2) las gráficas de la función de Wigner reducida para el estado $\left(n_{R}, n_{r}\right)=(0,1)$, cuya forma es,

$$
w(x, p)=\frac{8(2 \kappa+1)^{3 / 4}}{\pi \omega(1+\sqrt{2 \kappa+1})^{3}}\left(p^{2}+\omega^{2} x^{2}\right) e^{-\frac{2\left(p^{2}+\omega^{2} \sqrt{2 \kappa+1} x^{2}\right)}{\omega(1+\sqrt{2 \kappa+1})}} .
$$

En la Fig. (7.3) se muestra la gráfica de $s_{w}$, la entropía de Shannon de la función de Wigner reducida, $w(x, p)$. Las medidas de la localización en el espacio fase de dos partículas y en el espacio fase reducido de una partícula son consistentes en su comportamiento con el número cuántico de las coordenadas relativas, $n_{r}$, esto es, en ambos casos las distribuciones se deslocalizan con la excitación.

La suma entrópica (relación de incertidumbre) en el nivel de una partícula [112],

$$
s_{t}=s_{\rho}+s_{\pi}=\int d x d p \rho(x) \pi(p) \ln [\rho(x) \pi(p)] \geq 1+\ln \pi,
$$



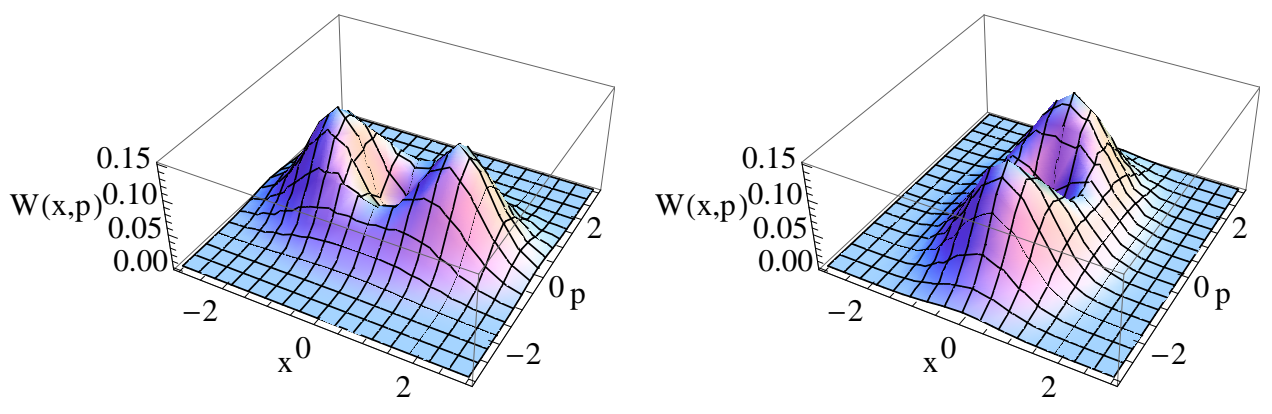

Figura 7.2: Gráficas 3D de la función de Wigner reducida de una partícula para el estado $\left(n_{R}, n_{r}\right)=(0,1)$ con $\omega=1$ y $\kappa=-0.4$ (izquierda), $\kappa=2.0$ (derecha).

puede verse como una medida de la localización en un espacio fase separable. La Fig. (7.3) muestra que el comportamiento de $s_{w}$ es consistente con el de $s_{t}$ como función de $n_{r}$; las distribuciones en espacio fase de dos partículas y reducidas de una partícula se deslocalizan conforme aumentamos $n_{r}$.

Las entropías de las distribuciones reducidas, $s_{\rho}$ y $s_{\pi}$, se comportan de manera similar a $s_{\Gamma}$ y $s_{\Pi}$, como funciones del potencial entre partículas. El comportamiento de estas cantidades se estudió en un capítulo anterior de esta tesis, por lo que nos enfocaremos en las medidas en espacio fase. Las entropías de las distribuciones en espacio fase reducidas, $s_{t}$ y $s_{w}$ dependen del potencial entre partículas en contraste con las entropías de las distribuciones de dos partículas, $s_{T}$ y $s_{W} . s_{t}$ y $s_{w}$ crecen con la magnitud del potencial, $|\kappa|$, como puede verse en la Fig. (7.3), indicando que las distribuciones se deslocalizan. La presencia del potencial se observa en las distribuciones de una partícula debido a la acción de "vestir" una partícula en la otra. 

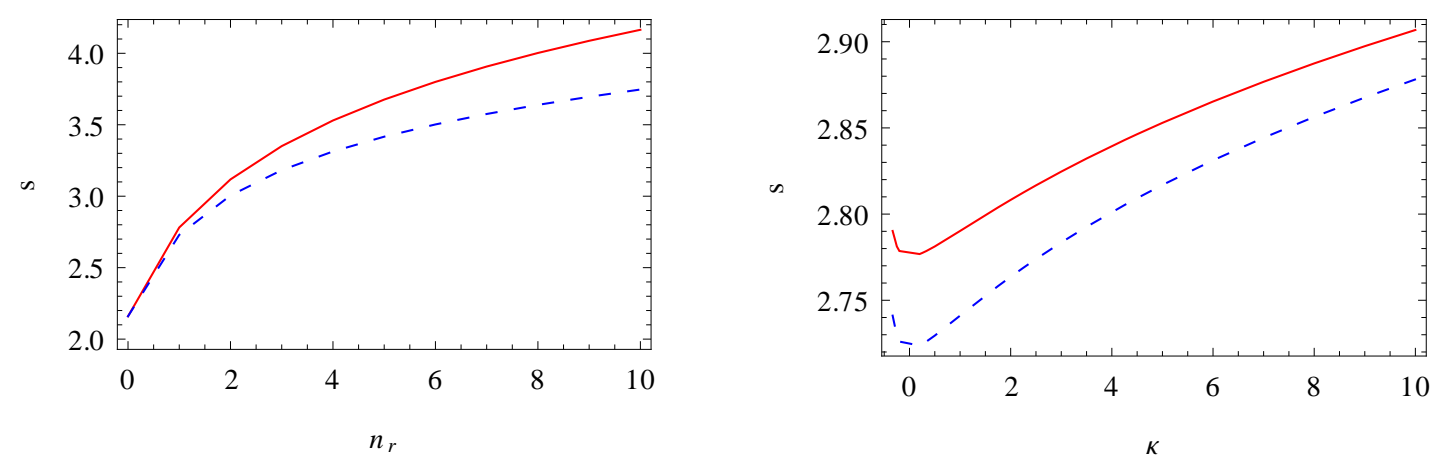

Figura 7.3: Suma entrópica de una partícula, $s_{t}$ (roja, línea sólida) y entropía de Shannon de la función de Wigner reducida de una partícula, $s_{w}$ (azul, línea discontinua). Izquierda: como función de $n_{r}$, para $n_{R}=0$ y $\kappa=-0.25$. Derecha: como funciones del potencial entre partículas, $\kappa$, para el estado excitado $(0,1)$.

\subsection{Correlaciones}

\subsubsection{Correlaciones entre partículas}

En esta sección vamos a reescribir algunas de las expresiones de la correlación que hemos discutido antes e introduciremos otras para generalizarlas utilizando la función de Wigner y para relacionarlas entre sí. La correlación entre las posiciones de las partículas o entre sus momentos puede estudiarse con la información mutua, definida por

$$
I_{x}=\int d x_{1} d x_{2} \Gamma\left(x_{1}, x_{2}\right) \ln \left[\frac{\Gamma\left(x_{1}, x_{2}\right)}{\rho\left(x_{1}\right) \rho\left(x_{2}\right)}\right]=2 s_{\rho}-s_{\Gamma} \geq 0,
$$

para posiciones, $\mathrm{y}$

$$
I_{p}=\int d p_{1} d p_{2} \Pi\left(p_{1}, p_{2}\right) \ln \left[\frac{\Pi\left(p_{1}, p_{2}\right)}{\pi\left(p_{1}\right) \pi\left(p_{2}\right)}\right]=2 s_{\pi}-s_{\Pi} \geq 0,
$$

para momentos. Ambas crecen con $n_{r}$, por lo tanto la correlación entre posiciones o entre momentos aumenta con al excitación. También tienen un comportamiento creciente $I_{x}$ e $I_{p}$ con la magnitud del potencial entre partículas. Su suma, $I_{x}+I_{p}$, 
puede interpretarse como una medida de las correlaciones entre partículas basada en una distribución de dos partículas en espacio fase separable,

$$
I_{x}+I_{p}=\int d x_{1} d p_{1} d x_{2} d p_{2} \Gamma\left(x_{1}, x_{2}\right) \Pi\left(p_{1}, p_{2}\right) \ln \left[\frac{\Gamma\left(x_{1}, x_{2}\right) \Pi\left(p_{1}, p_{2}\right)}{\rho\left(x_{1}\right) \rho\left(x_{2}\right) \pi\left(p_{1}\right) \pi\left(p_{2}\right)}\right],
$$

y puede generalizarse sustituyendo,

$$
\Gamma\left(x_{1}, x_{2}\right) \Pi\left(p_{1}, p_{2}\right) \rightarrow W\left(x_{1}, p_{1}, x_{2}, p_{2}\right), \quad \rho\left(x_{i}\right) \pi\left(p_{i}\right) \rightarrow w\left(x_{i}, p_{i}\right)
$$

Entonces tenemos,

$$
I_{a}=\int d x_{1} d p_{1} d x_{2} d p_{2} W\left(x_{1}, p_{1}, x_{2}, p_{2}\right) \ln \left[\frac{W\left(x_{1}, p_{1}, x_{2}, p_{2}\right)}{w\left(x_{1}, p_{1}\right) w\left(x_{2}, p_{2}\right)}\right]=2 s_{w}-s_{W},
$$

que mide la separabilidad de la función de Wigner de dos partículas en las funciones de Wigner marginales asaciadas a cada una de ellas. $I_{a}$ es en general un número complejo debido a la negatividad de $W\left(x_{1}, p_{1}, x_{2}, p_{2}\right)$, lo que vuelve necesario considerar tres posibles medidas cuantitativas de las correlaciones entre partículas,

$$
I_{a}=\left\{\begin{array}{l}
\operatorname{Re}\left[2 s_{w}-s_{W}\right] \\
\left|2 s_{w}-s_{W}\right| \\
\left|2 s_{w}\right|-\left|s_{W}\right| .
\end{array}\right.
$$

En la Fig. (7.4) se muestran estas cantidades como funciones de $n_{r}$. $R e\left[2 s_{w}-s_{W}\right]$ y $\left|2 s_{w}-s_{W}\right|$ crecen con $n_{r}$, comportamiento similar a $I_{x}+I_{p}$. Por otro lado, el comportamiento de $\left|2 s_{w}\right|-\left|s_{W}\right|$ es cualitativamente diferente, particularmente para estados altamente excitados. De las tres cantidades, esta es la que muestra menor correlación entre las partículas. En contraste con las otras dos cantidades $R e\left[2 s_{w}-s_{W}\right]$ no tiene en cuenta completamente las regiones negativas de la función de Wigner. Las otras dos cantidades toman en cuenta el total de las regiones negativas por lo que puede discutirse la contribución de éstas a la correlación entre partículas comparando con $R e\left[2 s_{w}-s_{W}\right]$; así, se observa que en un caso contribuyen a aumentar la correlación entre partículas $\left(\operatorname{Re}\left[2 s_{w}-s_{W}\right]<\left|2 s_{w}-s_{W}\right|\right)$, en tanto que en el otro caso la disminuyen 
$\left(R e\left[2 s_{w}-s_{W}\right]>\left|2 s_{w}\right|-\left|s_{W}\right|\right)$. Todas las cantidades aumentan con $|\kappa|$ debido a que son medidas de las correlaciones entre partículas. Note que $I_{a}$ depende en $\kappa$ a través de la dependencia de $s_{w}$.
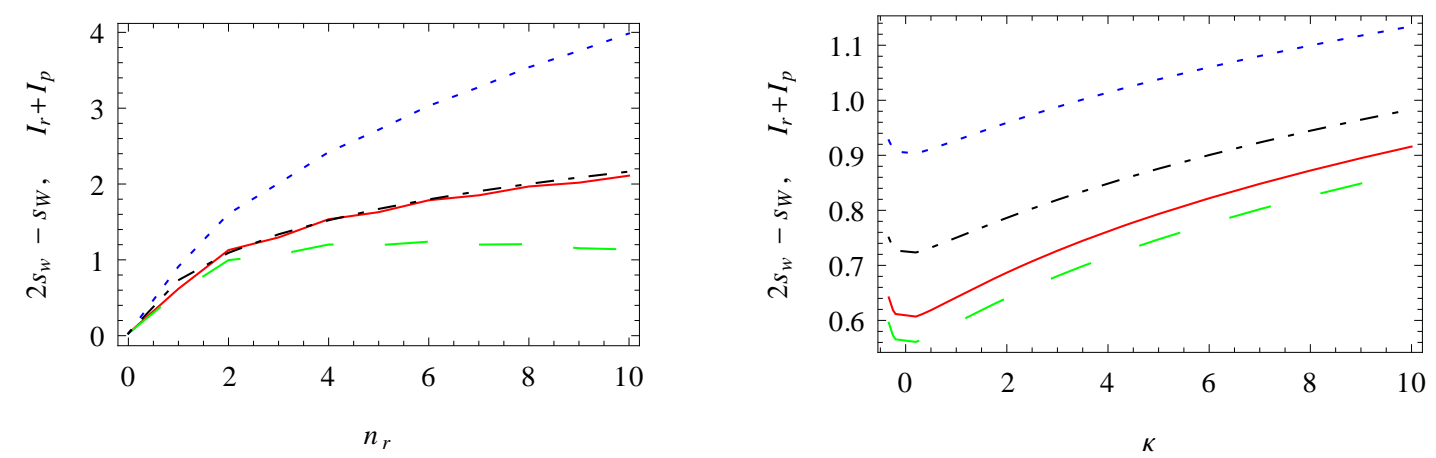

Figura 7.4: Correlación entre partículas, $\operatorname{Re}\left[2 s_{w}-s_{W}\right]$ (rojo, línea sólida), $\left|2 s_{w}\right|-\left|s_{W}\right|$ (verde, línea discontinua), y $\left|2 s_{w}-s_{W}\right|$ (azul, línea punteada). Izquierda: como funciones de $n_{r}$, para $n_{R}=0$ y $\kappa=-0.25$. Derecha: como funciones del potencial entre partículas, $\kappa$, para el estado excitado $(0,1)$. $I_{x}+I_{p}$ (gris, línea de rayas y puntos) también se muestra.

\subsubsection{Correlaciones totales}

El análisis de las correlaciones totales en el modelo puede realizarse definiendo

$$
I_{b}=\int d x_{1} d p_{1} d x_{2} d p_{2} W\left(x_{1}, p_{1}, x_{2}, p_{2}\right) \ln \left[\frac{W\left(x_{1}, p_{1}, x_{2}, p_{2}\right)}{\rho\left(x_{1}\right) \rho\left(x_{2}\right) \pi\left(p_{1}\right) \pi\left(p_{2}\right)}\right]=2 s_{t}-s_{W},
$$

que es una generalización de la distribución en espacio fase separable al sustituir

$$
\Gamma\left(x_{1}, x_{2}\right) \Pi\left(p_{1}, p_{2}\right) \rightarrow W\left(x_{1}, p_{1}, x_{2}, p_{2}\right) .
$$

Es diferente de $I_{a}$ en que el denominador del argumento logarítmico no son las funciones de Wigner reducidas de una partícula sino las densidades de una partícula. Por lo tanto, es una medida de la separabilidad de la función de Wigner de dos partículas en las densidades de cada una de las variables que caracterizan al sistema. Una vez más 
tenemos tres posibilidades,

$$
I_{b}=\left\{\begin{array}{l}
\operatorname{Re}\left[2 s_{t}-s_{W}\right] \\
\left|2 s_{t}-s_{W}\right| \\
\left|2 s_{t}\right|-\left|s_{W}\right| .
\end{array}\right.
$$

La Fig. (7.5) muestra que el comportamiento de estas tres cantidades es consistente; la correlación aumenta con la excitación y también con $|\kappa|$. Su dependencia en $\kappa$ es a través de $s_{t}$ porque $s_{W}$ no depende del potencial entre partículas.
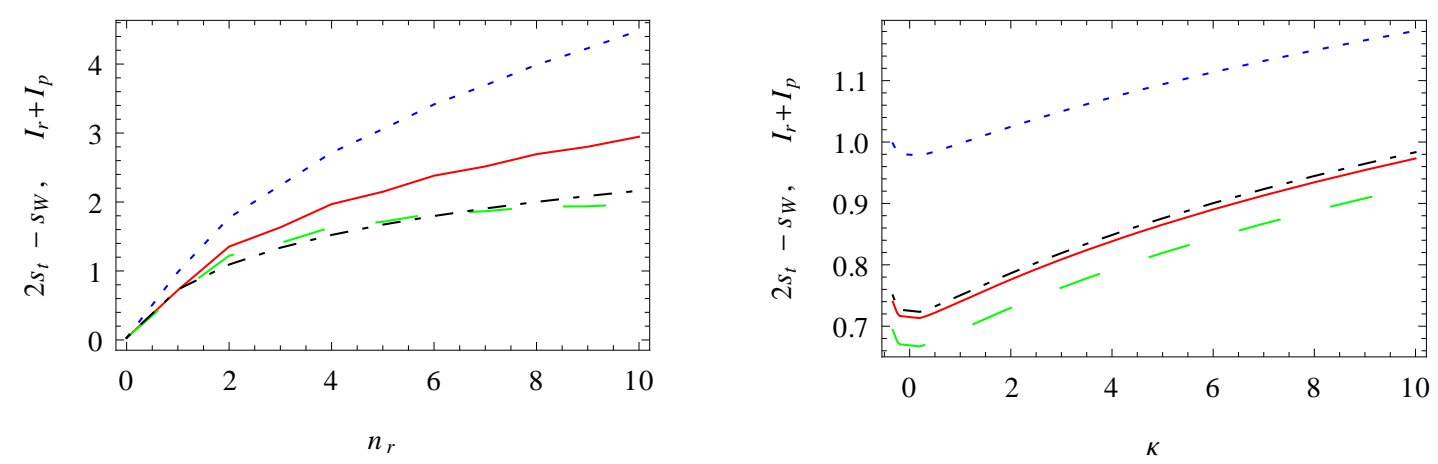

Figura 7.5: Correlación total, $R e\left[2 s_{t}-s_{W}\right]$ (rojo, línea sólida), $\left|2 s_{t}\right|-\left|s_{W}\right|$ (verde, línea discontinua), y $\left|2 s_{t}-s_{W}\right|$ (azul, línea punteada). Izquierda: como funciones de $n_{r}$, para $n_{R}=0$ y $\kappa=-0.25$. Derecha: como funciones del potencial entre partículas, $\kappa$, para el estado excitado $(0,1) . I_{x}+I_{p}$ (gris, línea de rayas y puntos) también se muestra.

\subsubsection{Correlación entre posición y momento}

\section{Nivel de dos partículas}

La correlación posición-momento en el nivel de dos partículas puede estudiarse utilizando,

$$
I_{x p}^{2}=\int d x_{1} d p_{1} d x_{2} d p_{2} W\left(x_{1}, p_{1}, x_{2}, p_{2}\right) \ln \left[\frac{W\left(x_{1}, p_{1}, x_{2}, p_{2}\right)}{\Gamma\left(x_{1}, x_{2}\right) \Pi\left(p_{1}, p_{2}\right)}\right]=s_{T}-s_{W}
$$


una medida de la no-separabilidad de la función de Wigner en las densidades de pares en cada espacio, que es la diferencia de la localización entre la función de distribución en un espacio fase separable $\left(s_{T}\right)$ y la no-separable $\left(s_{W}\right)$. Los valores complejos en $s_{W}$ nos conducen a las tres posibles medidas cuantitativas,

$$
I_{x p}^{2}=\left\{\begin{array}{l}
\operatorname{Re}\left[s_{T}-s_{W}\right] \\
\left|s_{T}-s_{W}\right| \\
\left|s_{T}\right|-\left|s_{W}\right| .
\end{array}\right.
$$

Estas tres cantidades se grafican en la Fig. (7.6) como funciones de $n_{r}$ y de $\kappa$. Las primeras dos, $R e\left[2 s_{w}-s_{W}\right]$ y $\left|2 s_{w}-s_{W}\right|$, aumentan con $n_{r}$, mientras que $\left|2 s_{w}\right|-\left|s_{W}\right|$ oscila. Este comportamiento es consistente con el observado en sistemas de una partícula [132]. $\left|2 s_{w}\right|-\left|s_{W}\right|$ es la que predice la menor correlación posición-momento. Existen algunos estados donde las definiciones empleadas asumen valores negativos, lo cual es provocado porque la distribución en espacio fase no-separable está más deslocalizada que la separable.
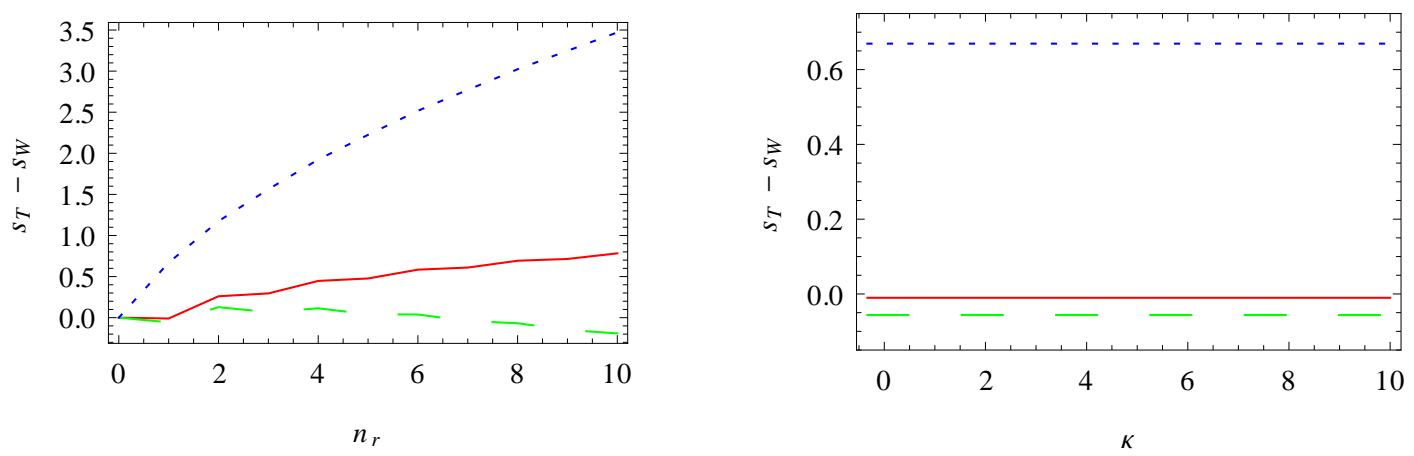

Figura 7.6: Correlación posición-momento en el nivel de dos partículas, $R e\left[s_{T}-s_{W}\right]$ (rojo, línea sólida), $\left|s_{T}\right|-\left|s_{W}\right|$ (verde, línea discontinua), y $\left|s_{T}-s_{W}\right|$ (azul, línea punteada). Izquierda: como funciones de $n_{r}$, para $n_{R}=0$ y $\kappa=-0.25$. Derecha: como funciones del potencial entre partículas, $\kappa$, para el estado excitado $(0,1)$.

Es importante anotar que como $s_{T}$ y $s_{W}$ son independientes del potencial entre 
partículas, $I_{x p}^{2}$ también lo es, tal como se puede apreciar en la Fig. (7.6). Entonces la correlación entre posición y momento en el nivel de dos partículas no se ve influenciada por el potencial entre partículas.

\section{Nivel de una partícula}

Para estudiar la correlación entre la posición y el momento en el nivel de una partícula definimos una medida de la separabilidad de la función de Wigner reducida en las densidades reducidas de una partícula,

$$
I_{x p}^{1}=\int d x d p w(x, p) \ln \left[\frac{w(x, p)}{\rho(x) \pi(p)}\right]=s_{t}-s_{w} .
$$

$I_{x p}^{1} \geq 0$ porque $w(x, p)$ es positivo-definida, lo cual significa que la función de Wigner reducida está siempre más localizada que su contraparte separable $\left(s_{w}<s_{t}\right)$. La Fig. (7.7) muestra $I_{x p}^{1}$ como función de $n_{r}$ y se observa que ésta aumenta con el número cuántico, por lo tanto, es consistente con dos de las cantidades que miden la correlación posición-momento en el nivel de dos partículas.
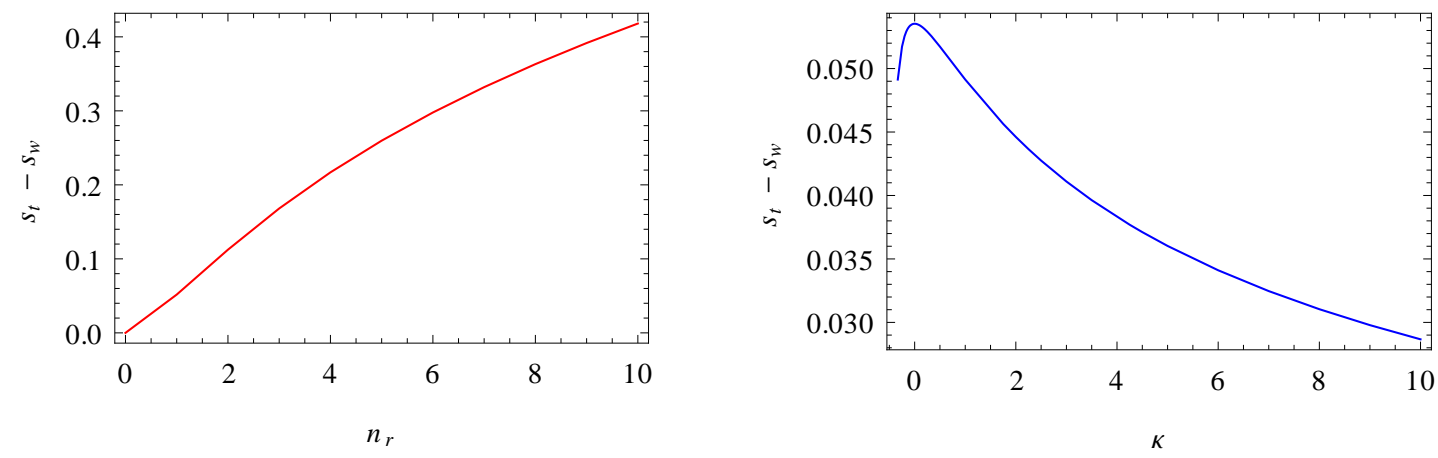

Figura 7.7: Correlación posición-momento en el nivel de una partícula, $s_{t}-s_{w}$. Izquierda (en rojo), como función de $n_{r}$, para $n_{R}=0$ y $\kappa=-0.25$. Derecha (en azul), como función del potencial entre partículas, $\kappa$, para el estado excitado $(0,1)$.

Una diferencia importante entre $I_{x p}^{1}$ y $I_{x p}^{2}$ es que la primera sí depende de $\kappa$ [Fig. (7.7)], entonces la correlación posición-momento en el nivel de una partícula se ve influenciada por la interacción entre partículas. La correlación disminuye con la magnitud 
del potencial en ambos regímenes (repulsivo y atractivo). Este comportamiento contrasta con el observado en el nivel de dos partículas (donde la interacción no tiene influencia en la correlación posición-momento) y se debe a que "vestir" una partícula en la otra guarda la información de la interacción en las densidades reducidas y ahora sí la magnitud de la interacción afecta la correlación posición-momento en el nivel de una partícula.

\subsubsection{Relaciones entre las medidas de correlación}

El propósito de esta sección es ilustrar que las correlaciones totales pueden expresarse en términos de sus componentes: 1) la correlación entre partículas y 2) la correlación posición-momento. Esperamos obtener información acerca de las correlaciones posición-momento eliminando las correlaciones entre partículas de las correlaciones totales $\left(I_{b}\right)$. Así, si restamos la medida generalizada de correlación entre partículas $I_{a}$ de $I_{b}$, tenemos,

$$
I_{b}-I_{a}=\left(2 s_{t}-s_{W}\right)-\left(2 s_{w}-s_{W}\right)=2 s_{t}-2 s_{w}=2 I_{x p}^{1},
$$

alternativamente, usando la correlación entre partículas medida en las distribuciones separables, podemos conseguir

$$
I_{b}-\left(I_{x}+I_{p}\right)=\left(2 s_{t}-s_{W}\right)-\left(2 s_{t}-s_{T}\right)=s_{T}-s_{W}=I_{x p}^{2} .
$$

Estas relaciones no son válidas si utilizamos como medida de correlación la norma de las cantidades involucradas (por ejemplo $\left|2 s_{w}-s_{W}\right|$ ). Sin embargo, son válidas si utilizamos la diferencia de las normas (por ejemplo $\left|2 s_{w}\right|-\left|s_{W}\right|$ ) o sólo el componente real de las medidas de correlación (por ejemplo $R e\left[2 s_{w}-s_{W}\right]$ ). Así, si utilizamos estas dos últimas alternativas para cuantificar la correlación, entonces la correlación posición-momento y la correlación entre partículas son separables según este esquema. 


\subsubsection{Correlación no local $x_{1} \rightarrow p_{2}$}

En el átomo de Moshinsky la correlación entre partículas (entre sus posiciones $x_{1}$ y $x_{2}$ o entre sus momentos $p_{2}$ y $p_{1}$ ) se debe al potencial y a la simetría de la función de onda. La correlación entre $p_{1}$ y $x_{1}$ o entre $x_{2}$ y $p_{2}$ se debe a la no conmutatividad de los operadores de posición y momento, (principio de incertidumbre). Las correlaciones en el modelo pueden resumirse en el siguiente esquema: $\left(x_{1} \rightarrow x_{2} \rightarrow p_{2} \rightarrow p_{1} \rightarrow x_{1}\right)$. Nos preguntamos a partir de éste ¿cómo estudiar la correlación no local entre $x_{1}$ y $p_{2}$ ?

Si queremos obtener información acerca de la correlación entre $x_{1}$ y $p_{2}$ podemos sustraer las correlaciones entre partículas [a través de $I_{a}$ ] y la correlación posición-momento en el nivel de una partícula $\left(I_{x p}^{1}\right)$ de las correlaciones totales $\left(I_{b}\right)$. Alternativamente, también podríamos restar $I_{x}+I_{p}$ y la correlación posición-momento en el nivel de una partícula $\left(I_{x p}^{1}\right)$.

Utilizando $I_{a}$ como medida de la correlación entre partículas, obtenemos,

$$
I_{b}-I_{a}-I_{x p}^{1}=2 I_{x p}^{1}-I_{x p}^{1}=I_{x p}^{1}
$$

El resultado de que la correlación $x_{1} \rightarrow p_{2}$ depende de la correlación posición-momento en el nivel de una partícula no es sorprendente porque en primer lugar $I_{x p}^{1}$ involucra el uso de una función de Wigner reducida donde la información acerca de $p_{2}$ ( $\left.\mathrm{y} x_{2}\right)$ se ha proyectado sobre las otras variables. $\mathrm{Y}$ en segundo lugar porque debido a la indistinguibilidad de las partículas la información acerca de $p_{2}$ debe ser la misma que para $p_{1}$.

Si ahora usamos $I_{x}+I_{p}$ como medida de la correlación entre partículas,

$$
\begin{aligned}
I_{b}-\left(I_{x}+I_{p}\right)-I_{x p}^{1} & =I_{x p}^{2}-I_{x p}^{1}=\left(s_{T}-s_{W}\right)-\left(s_{t}-s_{w}\right) \\
& =\left(s_{\Gamma}-s_{\rho}\right)+\left(s_{\Pi}-s_{\pi}\right)-\left(s_{W}-s_{w}\right) \\
& =s\left(x_{1} \mid x_{2}\right)+s\left(p_{1} \mid p_{2}\right)-s\left(x_{1} p_{1} \mid x_{2} p_{2}\right),
\end{aligned}
$$


donde las entropías condicionales se definen como,

$$
\begin{aligned}
s\left(x_{1} \mid x_{2}\right) & =-\int d x_{1} d x_{2} \Gamma\left(x_{1}, x_{2}\right) \ln \left[\frac{\Gamma\left(x_{1}, x_{2}\right)}{\rho\left(x_{2}\right)}\right], \\
s\left(p_{1} \mid p_{2}\right) & =-\int d p_{1} d p_{2} \Pi\left(p_{1}, p_{2}\right) \ln \left[\frac{\Pi\left(p_{1}, p_{2}\right)}{\pi\left(p_{2}\right)}\right], \\
s\left(x_{1} p_{1} \mid x_{2} p_{2}\right) & =-\int d x_{1} d p_{1} d x_{2} d p_{2} W\left(x_{1}, p_{1}, x_{2}, p_{2}\right) \ln \left[\frac{W\left(x_{1}, p_{1}, x_{2}, p_{2}\right)}{w\left(x_{2}, p_{2}\right)}\right] .
\end{aligned}
$$

Las entropías condicionales miden la incertidumbre en una(s) variable(s) dado que conocemos otra(s), por ejemplo, $s\left(x_{1} \mid x_{2}\right)$ mide la incertidumbre en $x_{1}$ una vez que se ha determinado $x_{2}$.

La Fig. (7.8) muestra las curvas obtenidas de la Ec. (7.22) usando la parte real y también la diferencia de las normas de las cantidades involucradas. Como una tendencia general ambas medidas aumentan (en magnitud) para estados excitados, pero tienen un comportamiento oscilatorio. En varios casos las dos medidas difieren en el signo (una es negativa y la otra positiva). Ambas disminuyen en valor absoluto con la magnitud del potencial, sea éste atractivo o repulsivo. Comparando las tendencias generales en la Fig. (7.8) con las curvas en la Fig. (7.7) $\left(s_{t}-s_{w}\right)$, puede observarse que las Ecs. (7.22) y (7.21) son consistentes en su comportamiento con $n_{r}$ y con $\kappa$.
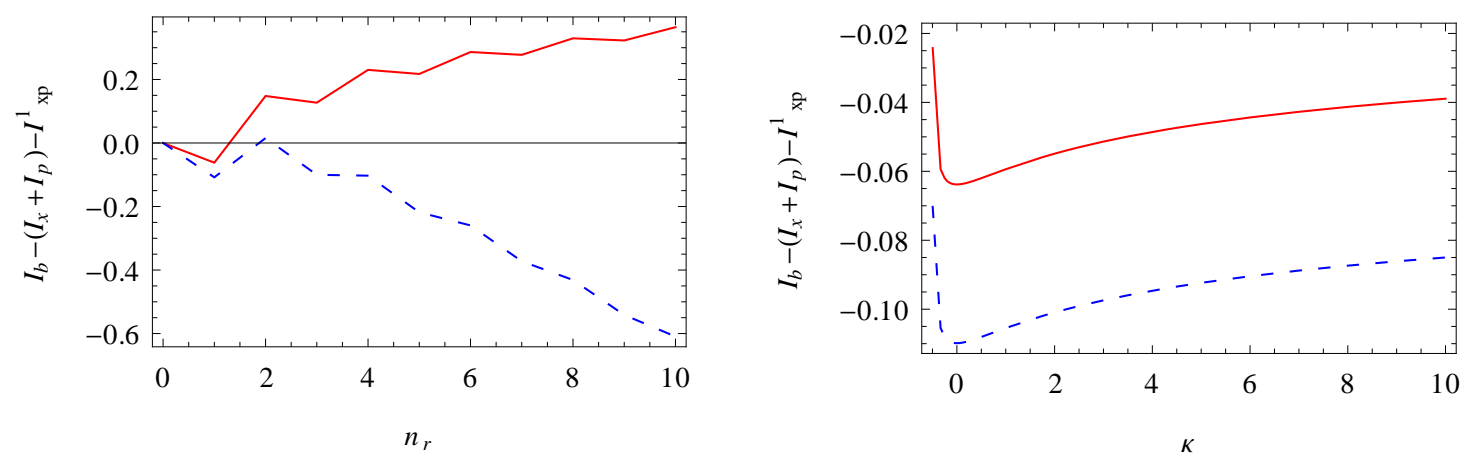

Figura 7.8: $I_{b}-\left(I_{x}+I_{p}\right)-I_{x p}^{1}$, con la parte real (rojo) y la diferencia de las normas (azul). Izquierda: como una función de $n_{r}$ para $n_{R}=0$ y $\kappa=-0.25$. Derecha: como función del potencial entre partículas, $\kappa$, para el estado excitado $(0,1)$.

Otra posibilidad para explorar estas correlaciones es partir del esquema de las cor- 
relaciones en el modelo ( $x_{1} \rightarrow x_{2} \rightarrow p_{2} \rightarrow p_{1} \rightarrow x_{1}$ ) y truncar la secuencia para examinar $\left(x_{1} \rightarrow x_{2} \rightarrow p_{2}\right)$. Esto es equivalente a sustraer de las correlaciones totales $\left(I_{b}\right)$, la correlación posición-momento en el nivel de una partícula $\left(I_{x p}^{1}\right)$ para eliminar la correlación $p_{1} \rightarrow x_{1}$, y la correlación entre los momentos de las partículas $\left(I_{p}\right)$ para eliminar la correlación $p_{2} \rightarrow p_{1}$,

$$
\begin{aligned}
I_{b}-I_{p}-I_{x p}^{1} & =\left(2 s_{t}-s_{W}\right)-\left(2 s_{\pi}-s_{\Pi}\right)-\left(s_{t}-s_{w}\right) \\
& =s_{\rho}+\left(s_{\Pi}-s_{\pi}\right)-\left(s_{W}-s_{w}\right) \\
& =s_{\rho}+s\left(p_{1} \mid p_{2}\right)-s\left(x_{1} p_{1} \mid x_{2} p_{2}\right) .
\end{aligned}
$$

La secuencia $\left(p_{2} \rightarrow p_{1} \rightarrow x_{1}\right)$ permite examinar la correlación $p_{2} \rightarrow x_{1}$ de manera similar al caso anterior con la única diferencia de sustraer $I_{x}$ en lugar de $I_{p}$,

$$
\begin{aligned}
I_{b}-I_{x}-I_{x p}^{1} & =\left(2 s_{t}-s_{W}\right)-\left(2 s_{\rho}-s_{\Gamma}\right)-\left(s_{t}-s_{w}\right) \\
& =s_{\pi}+\left(s_{\Gamma}-s_{\rho}\right)-\left(s_{W}-s_{w}\right) \\
& =s_{\pi}+s\left(x_{1} \mid x_{2}\right)-s\left(x_{1} p_{1} \mid x_{2} p_{2}\right) .
\end{aligned}
$$

En la Fig. (7.9) se muestran la parte real y las normas de los términos de las Ecs. (7.24) y (7.25) como funciones de $n_{r}$ y de $\kappa$. Se observa que su comportamiento con $n_{r}$ es consistente entre sí; sus partes reales aumentan con el número cuántico de las coordenadas relativas mientras las normas de las entropías tienen primero un comportamiento creciente y después decreciente para números cuánticos muy altos. También se observa que su comportamiento como funciones de $\kappa$ es consistente.

En la Fig (7.9) se observa que existen mínimos en las gráficas, demostrando que hay regiones donde al incrementar la magnitud del potencial se reduce la correlación entre $x_{1}$ and $p_{2}$ medida a través de este esquema, y regiones donde la correlación aumenta. Para la Ec. (7.24) el mínimo ocurre para un potencial entre partículas atractivo, en tanto que para la Ec. (7.25) éste ocurre en la región repulsiva. Todo 
este comportamiento evoca las medidas de correlación entre las posiciones o entre los momentos de las partículas, cuyas gráficas se muestran en otro capítulo de esta tesis.
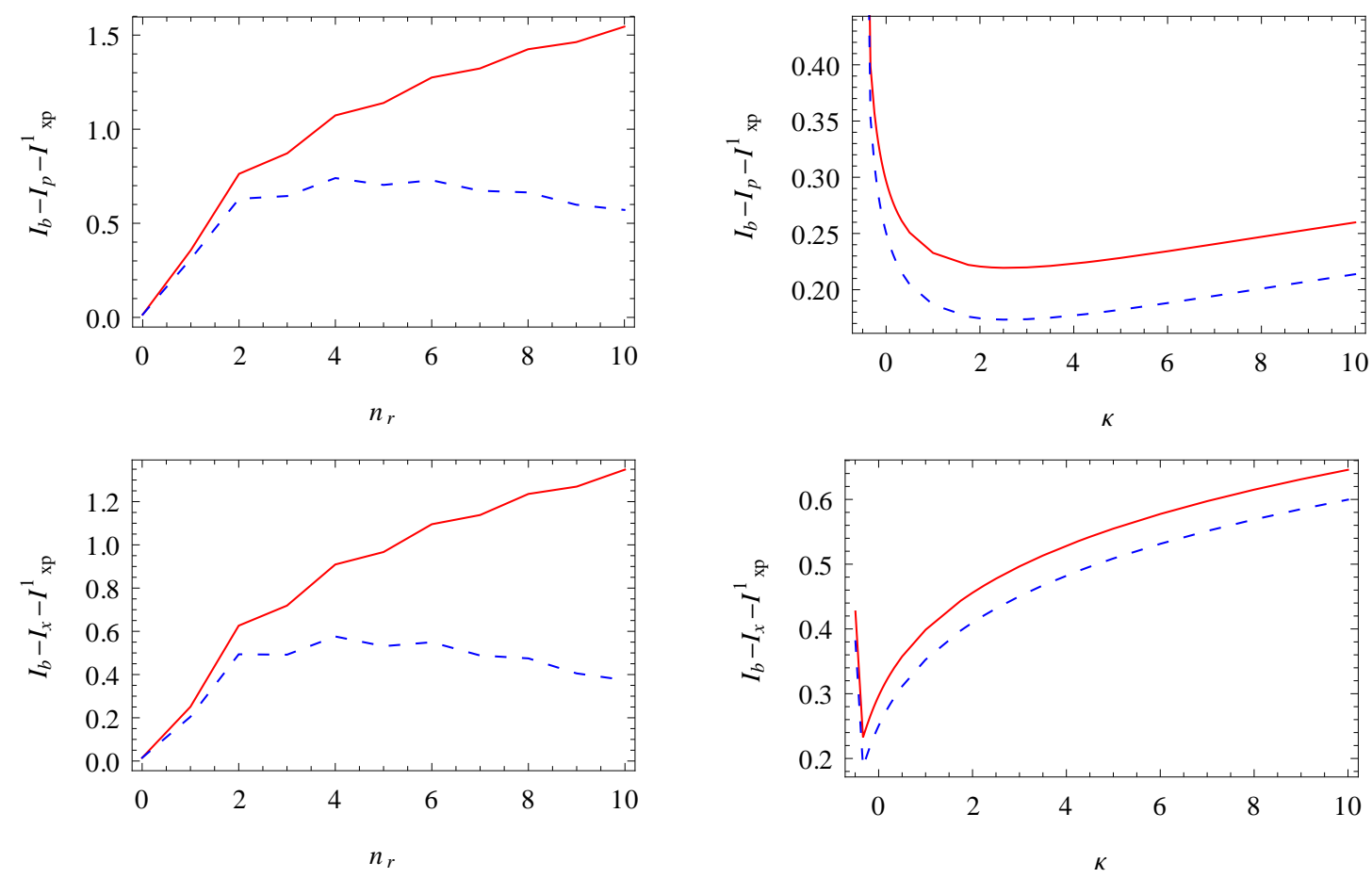

Figura 7.9: $I_{b}-I_{p}-I_{x p}^{1}$ (dos gráficas de arriba) y $I_{b}-I_{x}-I_{x p}^{1}$ (dos gráficas de abajo), con la parte real (rojo) y la diferencia de las normas (azul). Izquierda: como función de $n_{r}$ para $n_{R}=0$ y $\kappa=-0.25$. Derecha: como función del potencial entre partículas, $\kappa$, para el estado excitado $(0,1)$.

Como puede notarse al comparar las ecuaciones, hay una dependencia en el orden que elegimos en el esquema para analizar la correlación entre $x_{1}$ y $p_{2},\left(x_{1} \rightarrow x_{2} \rightarrow p_{2}\right)$ o $\left(p_{2} \rightarrow p_{1} \rightarrow x_{1}\right)$, la cuestión de hasta qué punto este orden influye en nuestra medida de esta correlación puede abordarse analizando la diferencia entre los esquemas. Así, conseguimos

$$
\begin{array}{r}
{\left[I_{b}-I_{p}-I_{x p}^{1}\right]-\left[I_{b}-I_{x}-I_{x p}^{1}\right]=\left(s_{\rho}-s_{\pi}\right)-\left[s\left(x_{1} \mid x_{2}\right)\right.} \\
\left.-s\left(p_{1} \mid p_{2}\right)\right] \\
=I_{x}-I_{p} .
\end{array}
$$


La diferencia entre $I_{x}$ e $I_{p}$ aumenta con $n_{r}$, y también incrementa con la magnitud del potencial entre partículas (atractivo o repulsivo), siendo cero $\left(I_{x}=I_{p}\right)$ cuando $\kappa=0$. Estas relaciones provocan la pregunta de hasta qué punto uno puede inferir información acerca de la correlación posición-momento a partir del análisis de las diferencias de la correlación entre las posiciones $\left(I_{x}\right)$ y entre los momentos $\left(I_{p}\right)$ de las partículas. Enfatizamos que esta discusión no es pertinente si utilizamos las normas de las cantidades, porque estas relaciones no serían válidas.

\subsection{Conclusiones}

Hemos investigado en el átomo de Moshinsky la localización en distribuciones separables y no separables en un espacio fase cuántico por medio del análisis de sus entropías de Shannon.

Las sumas entrópicas, relaciones informacionales de incertidumbre, pueden generalizarse sustituyendo las densidades de posición y momento con las correspondientes funciones de Wigner. En varios casos, la localización de las distribuciones en espacio fase separable y no separable se comportan consistentemente con respecto a las variaciones en el número cuántico de las coordenadas relativas.

Las entropías de pares de las distribuciones de espacio fase no dependen del potencial entre partículas, mentras que las entropías de las funciones reducidas sí dependen de éste. La interpretación de lo anterior es que el potencial entre partículas no tiene influencia en la localización en el espacio fase de dos partículas de este modelo. Otra diferencia entre los niveles de dos partículas y el reducido, es que las entropías de Shannon de dos partículas tienen valores complejos debido a que la función de Wigner presenta regiones negativas. Las entropías de las funciones de Wigner reducidas son números reales puesto que son positivas en todo el espacio.

En el capítulo estudiamos diferentes definiciones de la información mutua para abordar las correlaciones: totales, entre partículas y entre posición y momento. En el caso de las correlaciones entre partículas y totales, las correlaciones aumentan con la 
magnitud del potencial entre partículas y con el número cuántico de las coordenadas relativas.

La correlación posición-momento en los niveles de una y dos partículas generalmente se incrementan con el número cuántico de las coordenadas relativas. Sin embargo, la correlación posición-momento de dos partículas es independiente del potencial entre partículas mientras que la correlación posición-momento medida en las funciones reducidas sí depende del potencial. Esta correlación disminuye con la magnitud de los potenciales atractivo o repulsivo.

Así, hemos estudiado un esquema que permite separar las correlaciones entre partículas de las correlaciones posición-momento. 


\section{Correlación posición-momento en}

\section{un modelo de una partícula en una}

\section{caja con un potencial efectivo}

\subsection{El corral cuántico}

Este modelo bidimensional consiste en una partícula confinada con paredes de potencial infinito en un disco de radio unitario. Se ha utilizado para interpretar la densidad de estados de resultados experimentales [133, 134]. Si se usan coordenadas polares para escribir el Hamiltoniano, podemos encontrar las funciones de onda

$$
\psi_{n k}(r, \phi)=R_{n k}(r) \Phi_{k}(\phi),
$$

donde

$$
\begin{gathered}
R_{n k}(r)=\frac{2^{1 / 2}}{J_{|k|+1}\left(x_{n k}\right)} J_{k}\left(x_{n k} r\right), \\
\Phi_{k}(\phi)=\frac{1}{\sqrt{2 \pi}} e^{i k \phi},
\end{gathered}
$$

y $n=1,2,3, \cdots,|k|=0,1,2,3, \cdots . J_{k}$ es una función de Bessel de primer tipo [135] de orden- $k ;$ y $x_{n k}$ es el $n$-simo cero de esta función. De este modelo se han estudiado las entropías de Shannon en los espacios de posición y momento [136, 137].

Si se sustituye la función de onda de la Ec. (8.1) en la ecuación de Schrödinger, resolviendo para los ángulos obtenemos una ecuación radial, 


$$
-\frac{1}{r} \frac{d}{d r}\left(r \frac{d R_{n k}}{d r}\right)+\frac{k^{2}}{r^{2}} R_{n k}=\epsilon R_{n k}
$$

donde $\epsilon=\frac{2 m E}{\hbar^{2}}$. Si definimos la nueva función como

$$
u_{n k}(r)=\sqrt{r} R_{n k}(r)
$$

y la sustituimos en la Ec. (8.4) conseguimos,

$$
-\frac{d^{2} u_{n k}}{d r^{2}}+\frac{k^{2}-1 / 4}{r^{2}} u_{n k}=\epsilon u_{n k} .
$$

Esta ecuación puede interpretarse como una ecuación de Schrödinger unidimensional con un potencial efectivo dado por

$$
\frac{k^{2}-1 / 4}{r^{2}}
$$

Debido a que las $u$ 's son cero en los puntos $r=0$ y $r=1$, el modelo es equivalente a una partícula en una caja de longitud unitaria bajo la influencia de un potencial efectivo que resulta atractivo hacia el centro de la región de confinamiento para $k=0$, y repulsivo para todos los otros valores enteros de $k$. Con la metodología descrita en la parte introductoria de esta tesis podemos estudiar la influencia de los potenciales atractivos y repulsivos en la correlación posición-momento. Para racionalizar e interpretar estos resultados es posible contrastarlos con los que obtuvimos antes para el mismo modelo sin potencial, una partícula en una caja, PIAB [132, 2]. También podemos estudiar la localización en la función de Wigner utilizando la entropía de Shannon que hemos definido y además es posible contrastar los resultados con los de una partícula en una caja.

\subsection{Funciones de Wigner}

Las funciones de Wigner fueron calculadas numéricamente mediante la transformada de Weyl ,

$$
W_{n k}(r, p)=\frac{1}{\pi} \int d y u_{n k}(r+y) u_{n k}(r-y) e^{2 i p y}
$$


mientras que las entropías de Shannon mediante integración numérica de las Ecs. (4.45), (4.46) y (4.53). Las funciones $u_{n k}(r \pm y)$ deben ser cero fuera del intervalo $[0,1]$. Esta condición se satisface si

$$
0 \leq r+y \leq 1 \quad \text { y } \quad 0 \leq r-y \leq 1,
$$

lo que conduce a los límites de integración siguientes,

$$
\begin{array}{ll}
-r \leq y \leq+r, & \text { si } \quad 0 \leq r \leq \frac{1}{2} \\
-(1-r) \leq y \leq+(1-r), & \text { si } \quad \frac{1}{2}<r \leq 1
\end{array}
$$

Las entropías y la información mutua están dadas en nats. Un procedimiento para verificar nuestra integración numérica es calcular numéricamente con el mismo programa las funciones de Wigner de la partícula en la caja [138] y comparamos los valores que arrojó el cálculo con la expresión analítica; asimismo verificamos en cada caso la normalización de la función de Wigner.

En la Fig. (8.1) mostramos algunas gráficas de la función de Wigner para la partícula en una caja sin potencial y con potenciales atractivos y repulsivos. El primer renglón corresponde a la función de Wigner para el modelo PIAB para los números cuánticos $m=1,2,5$, en tanto que en los otros renglones mostramos las funciones de Wigner correspondientes a un potencial efectivo determinado por los valores $k=0,1,3$. Para hacer posible la comparación con el modelo PIAB, en cada renglón graficamos las funciones correspondientes a los números cuánticos $n=1,2,5$.

Si nos desplazamos sobre un renglón es posible observar tanto la influencia del número cuántico en la función de Wigner, como la manera en que cambia con la excitación. Por otra parte, desplazándose hacia abajo en cualquier columna se observa la introducción de un potencial atractivo (segunda entrada, $k=0$ ) y repulsivo (tercera y cuarta entradas, $k=1,3)$ y sus efectos en la función de Wigner. 

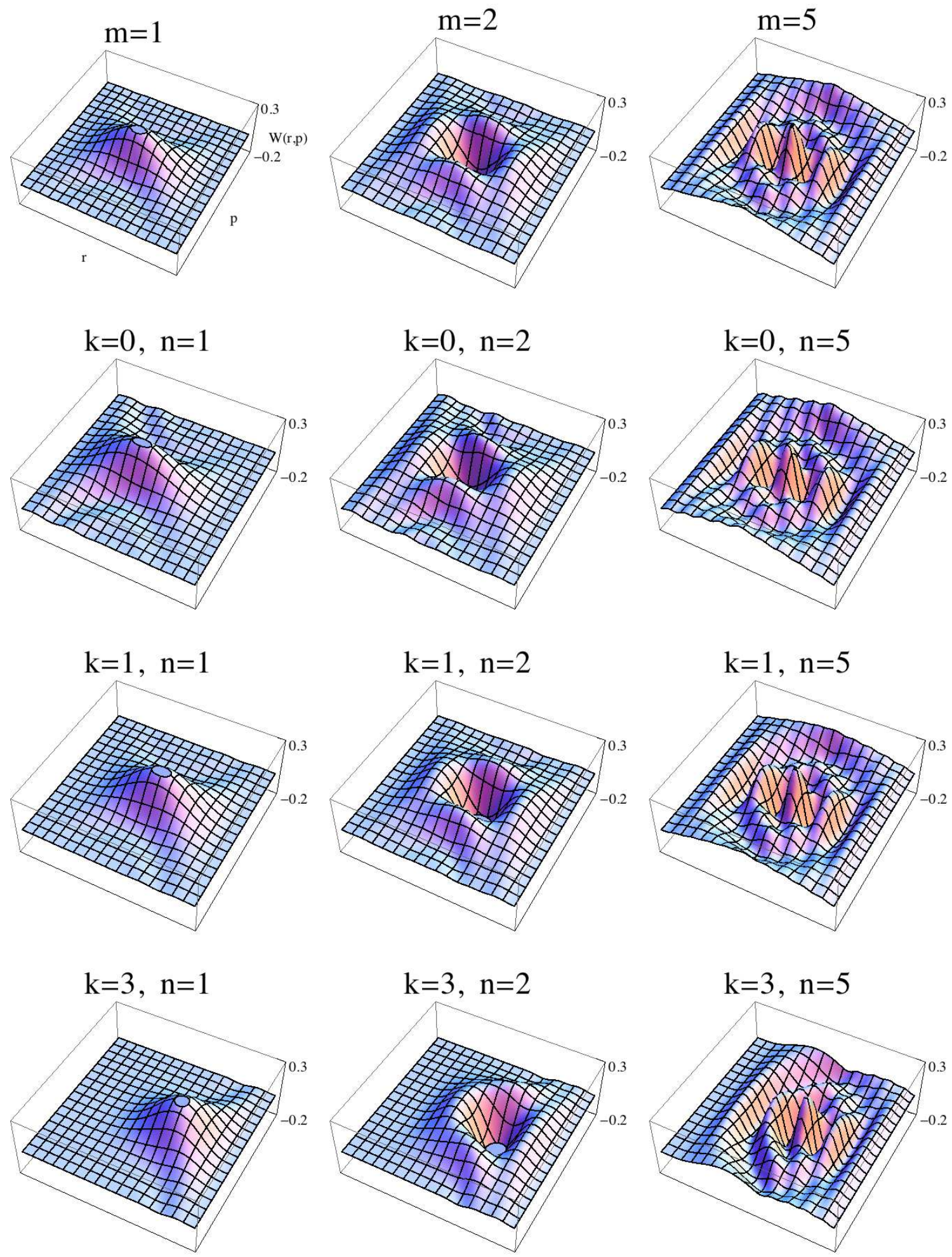

Figura 8.1: Perspectiva superior de las gráficas de la función de Wigner del modelo PIAB (primer renglón) y del modelo radial con $k=0$ (segundo renglón), $k=1$ (tercer renglón) y $k=$ 3 (cuarto renglón). Las columnas corresponden a $n(m)=1,2,5$. Las gráficas corresponden a valores de $r$ en el intervalo $(0,1)$ y valores de $p$ entre $(-15,15)$. 
El modelo radial es muy similar al modelo PIAB (primer renglón). Globalmente, los renglones comparten sus principales características. En las gráficas puede observarse, a lo largo de cada renglón, la aparición de más nodos conforme el sistema se excita hacia números cuánticos mayores. Aparentemente la estructura nodal se ve poco afectada por la introducción del potencial en el modelo, sin importar que éste sea repulsivo o atractivo.

Para observar los efectos del potencial atractivo comparamos la primera y segunda entrada en cada una de las columnas en las que se observa que la función de Wigner se encuentra ligeramente desplazada hacia el origen $(r=0)$ por los efectos de la atracción que ésta siente. Además, se observar que hay una ligera deslocalización en el eje $p$ para compensar la atracción en el eje $r$.

Por otro lado, para visualizar los efectos del potencial repulsivo comparamos los renglones 1 y 3 (ó 1 y 4). Se observa que el efecto del potencial sobre el eje $r$ "empuja" la función de Wigner hacia el extremo de la caja $(r=1)$ que resulta en su localización, en tanto que, también se advierte una cierta deslocalización a lo largo el eje $p$.

En este modelo es posible preguntarse por el efecto de la magnitud del potencial repulsivo, un análisis visual de este efecto puede lograrse comparando las últimas dos entradas de cada columna (correspondientes a $k=1,3$ ). El efecto de un potencial repulsivo más fuerte desplaza en mayor medida la función de Wigner sobre el eje $r$ hacia el extremo $r=1$, en tanto que, también es visible la diferencia en la deslocalización sobre el eje $p$.

En la siguiente sección haremos una caracterización cuantitativa de la localización/deslocalización que por ahora sólo hemos ejemplificado gráficamente. 

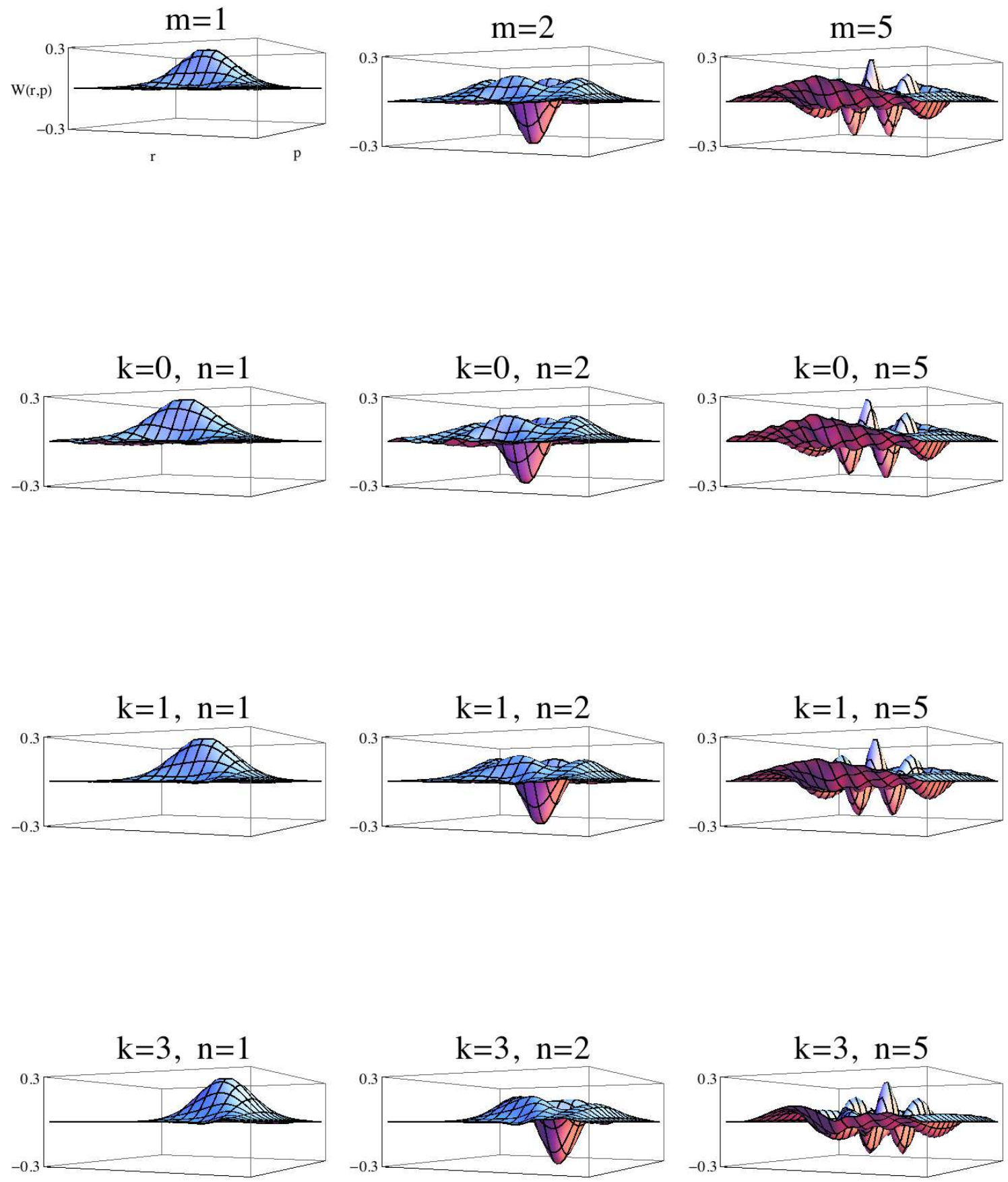

Figura 8.2: Perspectiva lateral de las gráficas de la función de Wigner del modelo PIAB (primer renglón) y del modelo radial con $k=0$ (segundo renglón), $k=1$ (tercer renglón) y $k=3$ (cuarto renglón). Las columnas corresponden a $n(m)=1,2,5$. El cero de la función de Wigner se encuentra a la mitad del eje vertical. Las gráficas corresponden a valores de $r$ en el intervalo $(0,1)$ y valores de $p$ entre $(-15,15)$. 
En el mismo sentido, la Fig. (8.2) muestra una perspectiva diferente de las funciones de Wigner, en la que puede observarse que el número de regiones negativas aumenta con la excitación. La columna correspondiente a $n=2$ muestra el desplazamiento tales regiones hacia el origen a causa del potencial atractivo, y se desplaza hacia la otra pared de la caja como efecto del potencial repulsivo.

Con el análisis de las gráficas no es posible plantear con amplitud la influencia del potencial en el aumento o disminución de las regiones negativas, por lo que, en la siguiente sección cuantificaremos tal influencia en las regiones negativas.

\subsubsection{Entropía de Shannon de la Función de Wigner}

Para analizar la localización en espacio fase de Wigner, utilizamos la entropía de Shannon de la función de Wigner, $s_{w}$. En la Fig. (8.3) mostramos $s_{w}$ como función del número cuántico principal, $n$, para $k=0,1$ y también como función de $n$ para el modelo PIAB. Como $s_{w}$ adquiere valores complejos se grafican las tres diferentes posibilidades para cuantificar la localización, $\operatorname{Re}\left[s_{w}\right], \operatorname{Im}\left[s_{w}\right]$ y $\left|s_{w}\right|$. Todas las cantidades son funciones crecientes de $n$ y $m$ cuyo comportamiento es consistente para cualquier valor de tales números cuánticos. En todas las medidas se puede observar cierta influ-

encia del potencial, no obstante en $R e\left[s_{w}\right]$ es menor la influencia; las diferencias entre las curvas para los diferentes potenciales son pequeñas. 

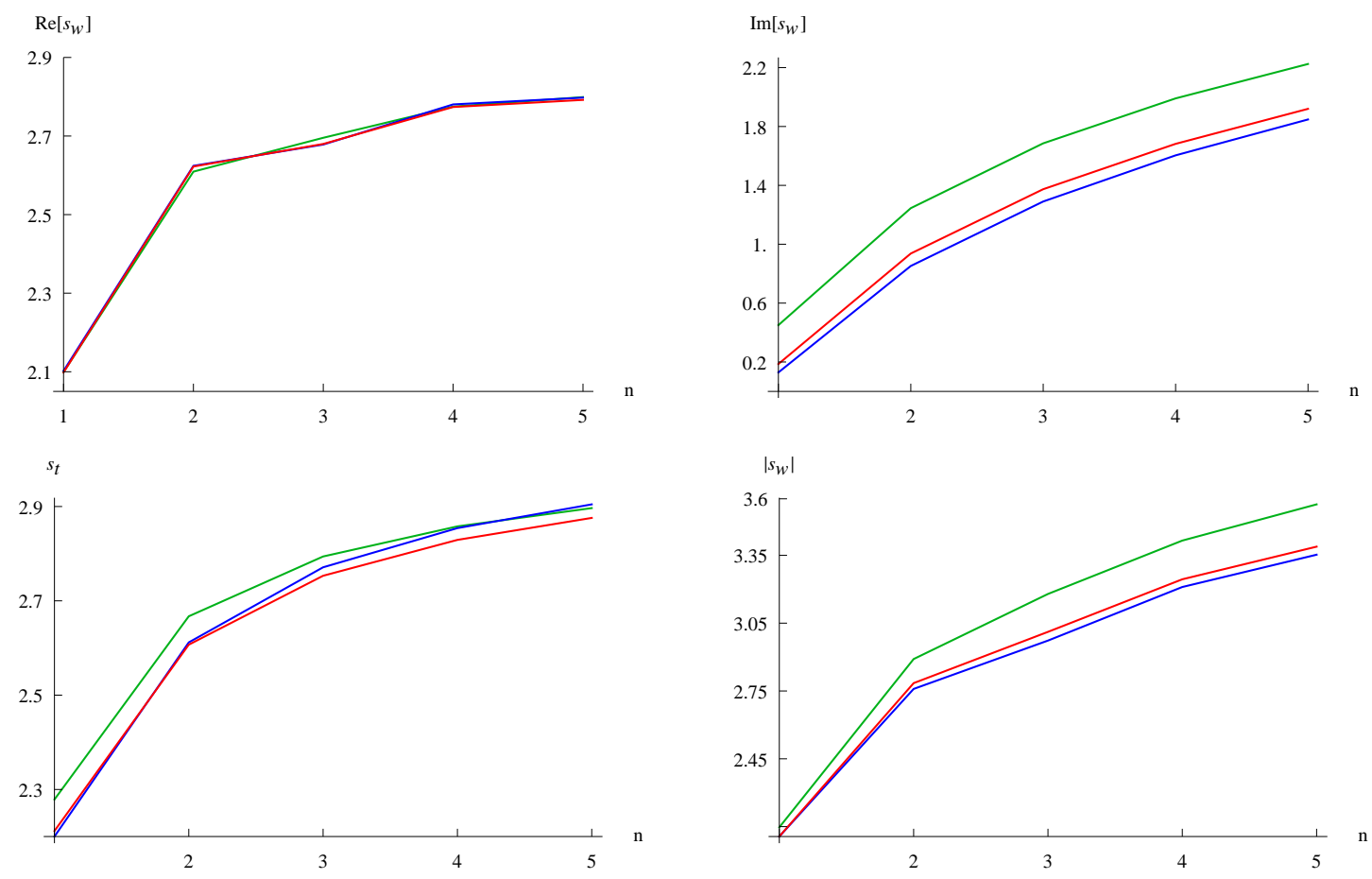

Figura 8.3: Gráficas de las tres posibilidades para la entropía de Shannon de la función de Wigner y de $s_{t}$ como funciones de $n(m): k=0$ (verde), PIAB (rojo), $k=1$ (azul).

En las gráficas para $\operatorname{Im}\left[s_{w}\right]$ y $\left|s_{w}\right|$ la curva para PIAB (valor cero del potencial) interpola entre los valores de $k=0$ (potencial atractivo) y $k=1$ (potencial repulsivo). La interpretación de estas curvas es que las funciones de Wigner se deslocalizan conforme aumenta la estructura nodal cuando crece $n$. Este comportamiento también se observa con otros potenciales cuyos valores ya hemos calculado, $k=3,5$.

El resultado de que $\operatorname{Im}\left[s_{w}\right]$ aumenta con $n$ muestra que las regiones negativas se hacen mayores conforme $n$ aumenta. También se observa que el volumen de las regiones negativas en el modelo PIAB tiene un valor intermedio entre aquel del potencial atractivo $(k=0)$ y aquel del repulsivo $(k>0)$.

Este orden entre los valores para $k=0$, PIAB y $k=1$ de las curvas correspondientes a $\operatorname{Im}\left[s_{w}\right]$ y $\left|s_{w}\right|$ se mantiene para todos los valores de $n$. Esto quiere decir que un potencial atractivo incrementa el volumen de las regiones negativas de la función de Wigner (comparando la curva para $k=0$ con la del modelo PIAB), mientras que 
un potencial repulsivo provoca la disminución del volumen de las regiones negativas (comparando las curvas para $k=1$ y PIAB). Así, un potencial atractivo deslocaliza la función de Wigner en tanto que un potencia repulsivo la localiza, cuando la referencia es el caso sin potencial (PIAB).

En la Fig. (8.3) también se muestran los valores correspondientes para la suma entrópica, $s_{t}$, una medida de la localización en el espacio fase, medido en una distribución separable y que puede ser comparado con las medidas discutidas en los párrafos anteriores. Es importante notar que mientras el comportamiento de $s_{t}$ es similar a las otras medidas, no preserva el orden de las curvas correspondientes a $k=0$, PIAB y $k=1$ como lo hacen las cantidades $\operatorname{Im}\left[s_{w}\right]$ y $\left|s_{w}\right|$. Existen puntos donde las curvas se cruzan (como ocurre con $\operatorname{Re}\left[s_{w}\right]$ ). Así, puede observarse que las medidas que incorporan las regiones negativas de la función de Wigner respetan el orden relativo mencionado, en tanto que $s_{t}$, que proviene de una distribución en espacio fase separable y no negativa, no respeta tal orden.

En la Fig. (8.4) mostramos las gráficas de las tres medidas de correlación posibles como funciones de $k$. Se observa que $\operatorname{Re}\left[s_{w}\right]$ no muestra mucha sensibilidad al potencial. Por otra parte, $\operatorname{Im}\left[s_{w}\right]$, y en menor medida $\left|s_{w}\right|$ muestran claramente cómo cambia la forma de la curva yendo desde $k=0$ hacia $k>0$. Entonces, la diferencia entre los potenciales atractivos y repulsivos se manifiesta en la parte imaginaria de $s_{w}$ (que también está incluida en $\left|s_{w}\right|$. 

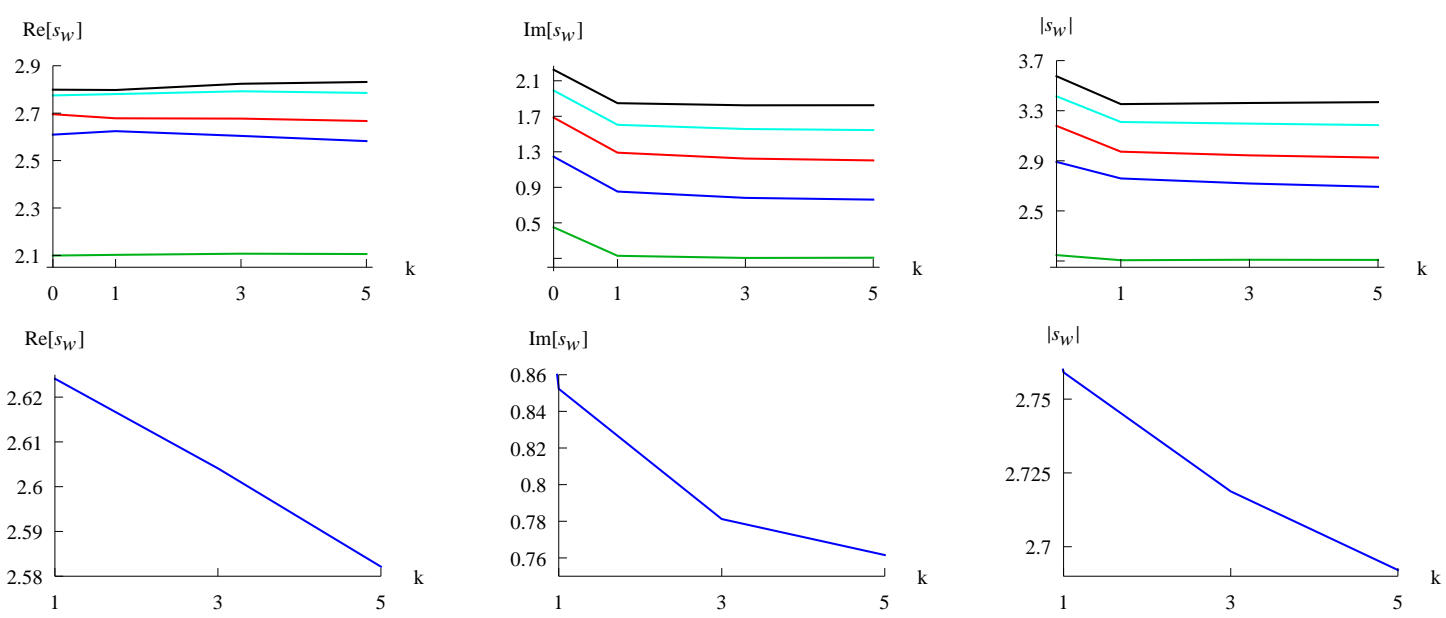

Figura 8.4: Gráficas de las tres posibilidades para la entropía de Shannon de la función de Wigner como funciones de $k: n=1$ (verde), $n=2$ (azul), $n=3$ (rojo), $n=4$ (azul claro), $n=5$ (negro). Hemos amplificado la escala de las tres curvas de abajo, corresponden a $n=2$ con potenciales repulsivos $k \geq 1$.

Las curvas pertenecientes a $\operatorname{Im}\left[s_{w}\right]$ y $\left|s_{w}\right|$ presentan poca variación para los diferentes valores $k \geq 1$. Esto sugiere que la magnitud o intensidad del potencial repulsivo no es un factor dominante en el cambio de las regiones negativas de la función de Wigner y en la localización de la distribución completa. Para interpretar este comportamiento regresaremos a las gráficas de la función de Wigner en la Fig. (8.1), en las que el ejemplo más claro son las dos últimas entradas de la primera columna. El potencial repulsivo más fuerte en la última entrada comprime la función de Wigner hacia la frontera en la dirección $r$. Esta compresión resulta en un ensanchamiento de la función en la dirección $p$ que puede atribuirse al principio de incertidumbre. El compromiso entre estos dos comportamientos provoca que haya una influencia neta pequeña en la localización de la función de Wigner como una totalidad.

El último renglón de la Fig. (8.4) muestra las gráficas correspondientes a $n=2$ para cada una de las cantidades utilizando una amplificación de la escala. Puede observarse que todas las cantidades decrecen conforme $k$ aumenta (potenciales repulsivos más fuertes). Enfatizamos que este comportamiento para $n=2$ se observó también en varios 
grupos de $n^{\prime} s$. Existen, sin embargo, $n^{\prime} s$ donde el comportamiento es creciente con $k$, por ejemplo para $n=5$ las cantidades $R e\left[s_{w}\right]$ y $\left|s_{w}\right|$ muestran este comportamiento. Este comportamiento creciente también ha sido observado para $s_{t}$ [137].

\subsubsection{Información Mutua}

La Fig. (8.5) muestra las gráficas de las tres definiciones de la información mutua, $R e\left[s_{t}-s_{w}\right],\left|s_{t}-s_{w}\right|$ y $\left|s_{t}\right|-\left|s_{w}\right|$ como funciones de $n$ para $k=0$, PIAB y $k=1$. Es importante notar que el comportamiento es similar al modelo PIAB en las tres definiciones. Para las últimas dos definiciones que incorporan las partes imaginarias, la interpretación es que la correlación posición-momento aumenta en magnitud con $n$ como en el modelo PIAB. También, la curva para PIAB interpola perfectamente entre las curvas para $k=0$ y $k=1$. Para las curvas correspondientes a $R e\left[s_{t}-s_{w}\right]$, la interpretación es menos transparente porque la curva aumenta y disminuye sin una tendencia clara.
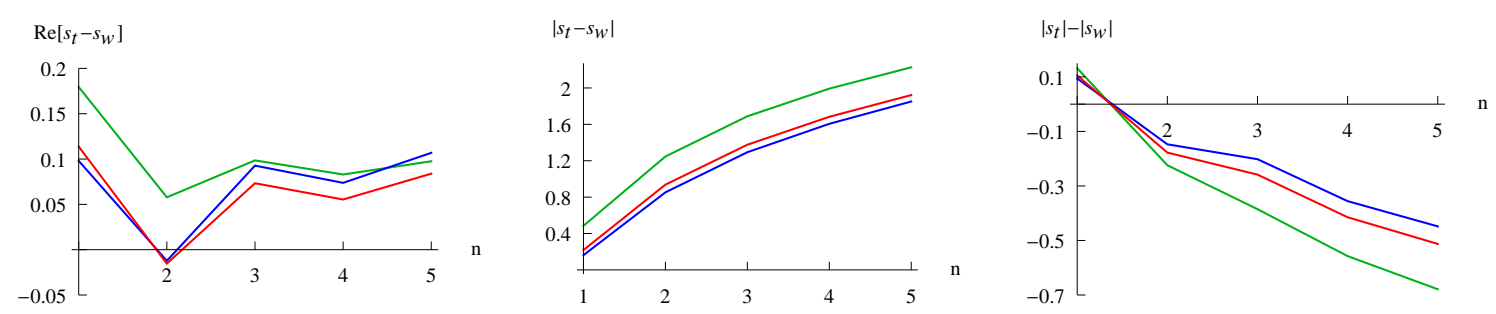

Figura 8.5: Gráficas de las tres posibilidades para la Información mutua como funciones de $n(m): k=0$ (verde), PIAB (rojo), $k=1$ (azul).

En las dos últimas gráficas, la magnitud de la correlación es mayor para la curva correspondiente a $k=0$ que la del modelo PIAB. Por tanto, el potencial atractivo incrementa la magnitud de la correlación posición-momento. Por otra parte, la magnitud de la correlación es menor para $k=1$ que para PIAB. Entonces el potencial repulsivo disminuye la correlación posición-momento.

El componente imaginario de $I_{r p}, \operatorname{Im}\left[s_{t}-s_{w}\right]$, puede también considerarse como 
una medida de correlación, tal componente es $-\operatorname{Im}\left[s_{w}\right]$ y es el negativo de la curva presentada en la Fig. (8.3). Examinando esta curva, la interpretación sería que la magnitud de la correlación aumenta con $n$. Esto es consistente con $\left|s_{t}-s_{w}\right| \mathrm{y}\left|s_{t}\right|-\left|s_{w}\right|$ en la interpretación de los efectos de los potenciales sobre la magnitud de la correlación.

La Fig. (8.6) presenta las curvas como funciones de $k$. Todas las curvas muestran una distinción entre los potenciales atractivo y repulsivo. La interpretación es que la magnitud de la correlación es mayor para potenciales atractivos que para repulsivos. Existen también diferencias entre las medidas de correlación, en $\left|s_{t}-s_{w}\right|$ la correlación posición-momento no se ve muy afectada por el potencial repulsivo $(k \geq 1)$ mientras que para las otras medidas la interpretación es que la magnitud de la correlación aumenta $\left(\operatorname{Re}\left[s_{t}-s_{w}\right]\right)$ o disminuye $\left(\left|s_{t}\right|-\left|s_{w}\right|\right)$ con la intensidad del potencial.
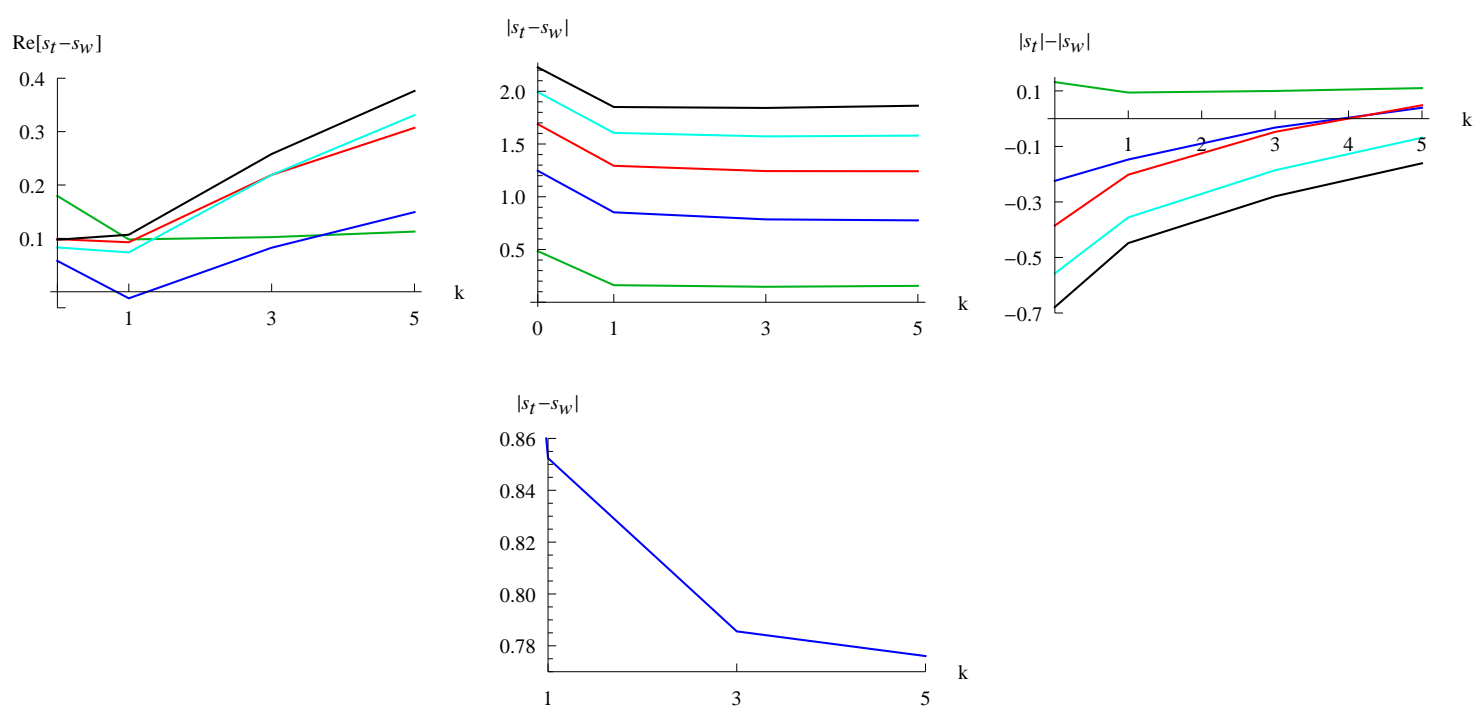

Figura 8.6: Gráficas de las tres posibilidades para la Información mutua como funciones de $k: n=1$ (verde), $n=2$ (azul), $n=3$ (rojo), $n=4$ (azul claro), $n=5$ (negro). La curva de abajo corresponde a $\left|s_{t}-s_{w}\right|$, para $n=2$ con potenciales repulsivos $k \geq 1$, hemos amplificado la escala.

La curva $\left|s_{t}-s_{w}\right|$ para $n=2$ se presenta en la parte de abajo de la Fig. (8.6) con una escala amplificada. Con esta escala, puede advertirse que $\left|s_{t}-s_{w}\right|$ disminuye y 
por lo tanto la correlación disminuye con la intensidad del potencial repulsivo. Este comportamiento es similar al de todas las curvas $\left|s_{t}\right|-\left|s_{w}\right|$. Estos resultados para $n=2$ también son válidos para los puntos $n=3$, pero no para los otros. Por otro lado, una disminución de los valores en $k=1$ y $k=3$ se observa para todos los valores estudiados de $n$.

\subsection{Conclusiones}

Podemos estudiar los efectos de un potencial efectivo repulsivo o atractivo en el comportamiento de las funciones de Wigner en una partícula en la caja por medio de las soluciones de la ecuación radial de Schrödinger para una partícula en un corral cuántico.

Estudiamos la localización/deslocalización de la función de Wigner con la entropía de Shannon. Mostramos que la entropía de Shannon se incrementa con el número cuántico, $n$ para ambos potenciales, atractivo y repulsivo, de manera similar al caso de potencial-cero (PIAB). La parte imaginaria de la entropía aumenta con $n$, lo que ilustra que el volumen de las regiones negativas de la función de Wigner se incrementa con el estado cuántico.

Además, la curva PIAB (potencial-cero) interpola entre el potencial atractivo $(k=$ 0) y el repulsivo $(k=1)$. La interpretación de esto es que potencial atractivo provoca el aumento del volumen negativo de la función de Wigner, en tanto que el potencial repulsivo provoca su disminución. Este análisis de la entropía de Shannon contra $k$ demuestra que la entropía detecta la diferencia entre un potencial atractivo y uno repulsivo.

La información mutua entre posición y momento se utiliza para estudiar la correlación estadística entre esas variables. Esta correlación se incrementa con el número cuántico y es consistente con el modelo PIAB. Además, los valores del modelo PIAB interpolan entre los casos $k=0$ y $k=1$, permitiendo una interpretación en función del signo del potencial. La presencia de un potencial atractivo incrementa la magnitud de esta correlación mientras que un potencial repulsivo disminuye esta correlación. 
Como una función de $k$, los resultados muestran una clara distinción entre los potenciales repulsivo y atractivo. Se observa que la magnitud de la correlación disminuye con el aumento del potencial repulsivo. Enfatizamos la importortancia de los componentes imaginarios de estas medidas informacionales, correspondientes al volumen de las regiones negativas de la función de Wigner. 


\section{Localización en espacio de}

\section{posición, momento y espacio fase, $y$ correlación posición-momento en el oscilador armónico confinado}

El oscilador armónico confinado puede visualizarse como otro modelo de una partícula en una caja con un potencial. Y también puede considerarse como un modelo intermedio entre la partícula en la caja y el oscilador armónico [139]. El Hamiltoniano del modelo es

$$
\hat{H}=-\frac{1}{2} \hat{p}^{2}+V(x),
$$

donde

$$
V(x)= \begin{cases}\frac{\omega^{2}}{2} \hat{x}^{2}, & \text { si }-a<x<a \\ \infty, & \text { si }|x|>a\end{cases}
$$

Las soluciones de este Hamiltoniano son analíticas y conocidas, para $n$ par,

$$
\psi_{n}(x)=A_{n} e^{-x^{2} / 2}{ }_{1} F_{1}\left(\frac{1}{4}-\frac{E_{n}}{2}, \frac{1}{2}, x^{2}\right)
$$

y para $n$ impar,

$$
\psi_{n}(x)=B_{n} e^{-x^{2} / 2}{ }_{1} F_{1}\left(\frac{3}{4}-\frac{E_{n}}{2}, \frac{3}{2}, x^{2}\right)
$$


donde $A_{n}$ y $B_{n}$ son constantes de normalización, y ${ }_{1} F_{1}(a, b, x)$ es la función hipergeométrica confluente de Kummer y $E_{n}$ son las energías del oscilador. Usualmente, para encontrar las $E_{n}$, se fija el radio de confinamiento y se resuelve, numéricamente, para la condición de confinamiento,

$$
{ }_{1} F_{1}\left(\frac{1}{4}-\frac{E_{n}}{2}, \frac{1}{2}, x_{c}^{2}\right)=0 \quad, \quad{ }_{1} F_{1}\left(\frac{3}{4}-\frac{E_{n}}{2}, \frac{3}{2}, x_{c}^{2}\right)=0,
$$

para el caso par e impar, respectivamente. En general debemos considerar que la función hipergeométrica confluente, ${ }_{1} F_{1}$, es una serie, convergente, infinita de potencias de $x^{2}$, y por ello las funciones de onda en el espacio de momento (Transformada de Fourier) y las funciones de Wigner (Transformada de Weyl) deben obtenerse por integración numérica de la Ec. (2.5), así como las funciones de Wigner de la Ec. (3.13)

Una desventaja para transformar la función al espacio de momento (Transformada de Dirac-Fourier) o al espacio fase (Transformada de Weyl) es que la función hipergeométrica confluente, ${ }_{1} F_{1}$, es una serie, convergente, infinita de potencias de $x^{2}$; en este caso la transformación debe ser numérica y dado que estamos interesados en el cálculo de la entropía, que se obtiene integrando numéricamente también, enfrentamos el problema de superponer dos aproximaciones numéricas.

Para ciertas energías, la función de onda en el espacio de momento y la función de Wigner son analíticas por lo que pueden usarse para verificar las tendencias que encontramos numéricamente. Dedicaremos una sección a estos resultados más adelante.

El modelo se ha discutido anteriormente por ser uno de los casos de solución analítica cuando el sistema está confinado, para abordarlo desde diferentes perspectivas $[139,140,141,142,143,144,145,146,147,148,149,150,151]$. 


\subsection{Localización}

\subsubsection{Espacio de posición}

En la Fig. (9.1) se muestra el comportamiento creciente de la desviación estándar con el tamaño de la caja, a, indicando que la partícula se deslocaliza cuando tiene más espacio disponible.

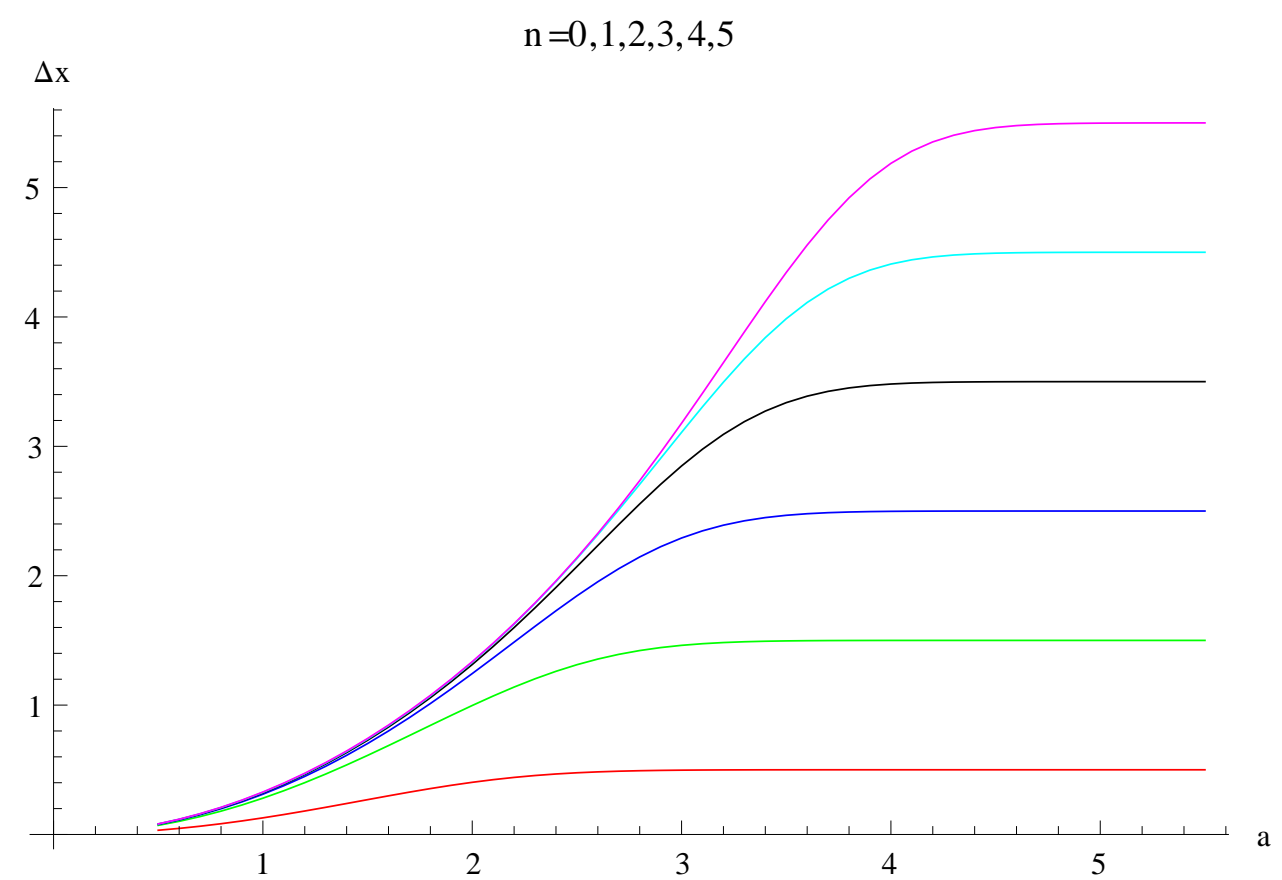

Figura 9.1: $\Delta x$ como función de $a$ para diferentes valores de $n, n=0$ es rojo y los otros valores son ascendentes conforme $n$ crece.

La entropía en el espacio de posición se ha estudiado anteriormente [141]. En la Fig. (9.2) se muestran las gráficas de las entropías en el espacio de posición correspondientes a $n=0,1,2,3,4,5$, en este modelo el número cuántico se verifica observando la cantidad de nodos que tiene la función de onda para una energía dada. Como en el caso de $\Delta x$ se observa que la partícula se deslocaliza cuando tiene más espacio disponible.

Para valores pequeños de $a$ puede advertirse que $s_{x}$ no depende apreciablemente de $n$, evocando el comportamiento de una partícula en una caja $[152,83]$, conforme 
aumentamos el tamaño de la caja van distinguiéndose las curvas correspondientes a los diferentes números cuánticos, similar al oscilador armónico.

En todos los casos ocurre que $s_{x}^{P I A B}(a=1)<s_{x_{n}}^{C H O}<s_{x_{n}}^{H O}(\omega=1)$. El subíndice para PIAB se ha suprimido por su independencia del número cuántico, aunque sí depende del tamaño de la caja. Es notable este comportamiento porque en el modelo CHO variamos el valor de $a$ (y por tanto el de $\omega$ ) y en los límites de $a$ pequeño y grande conseguimos los valores correspondientes a la caja con $a=1$ y al oscilador armónico $\operatorname{con} \omega=1$.

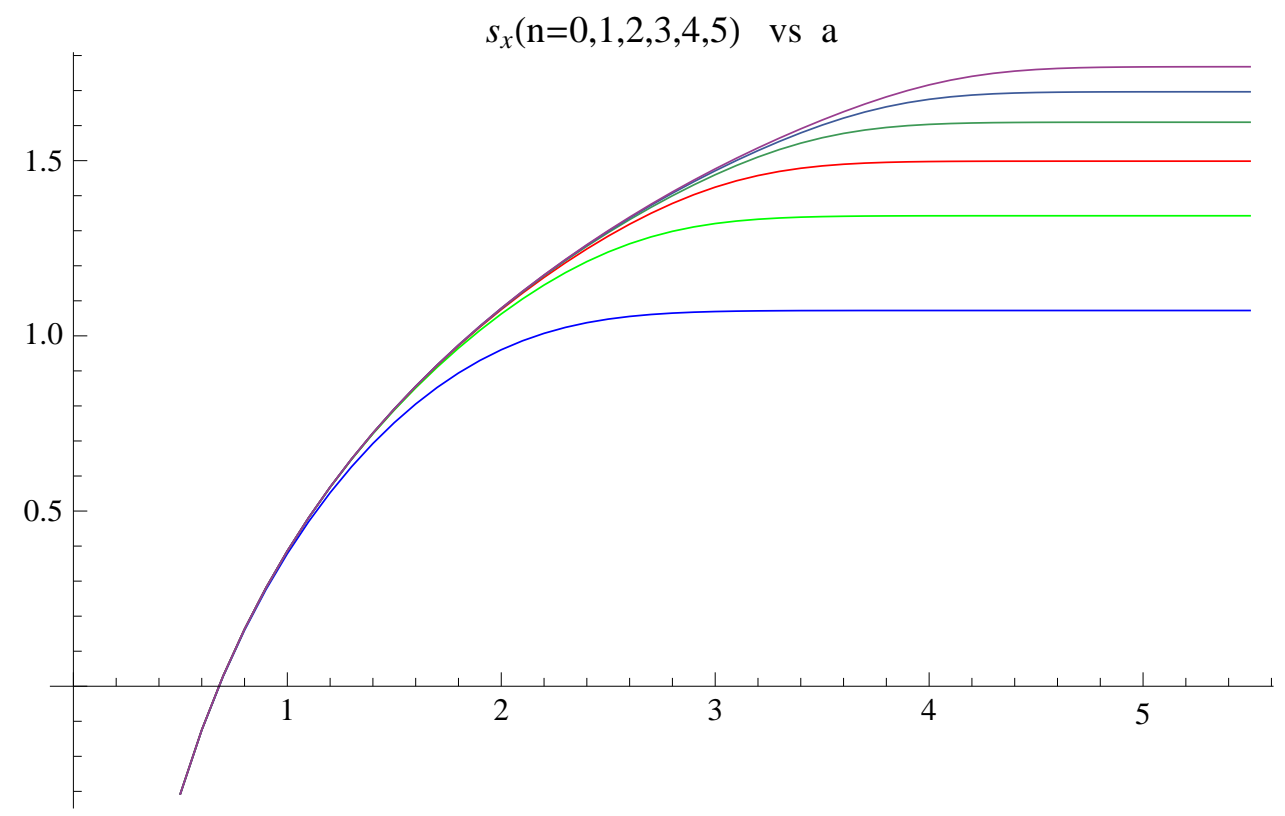

Figura 9.2: $s_{x}$ como función de $a$ para diferentes números cuánticos, $n=0$ es azul y los otros valores son ascendentes conforme $n$ crece.

En cuanto a la dependencia con el número cuántico, se muestra en la Fig. (9.3) que para $a=1 / \sqrt{2}$ en $s_{x}$ no hay dependencia apreciable con $n$, como ocurre con $s_{x}^{P I A B}$. Conforme $a$ aumenta, puede empezar a observarse la dependencia con $n$, lo que demuestra también la transición de un modelo a otro. 


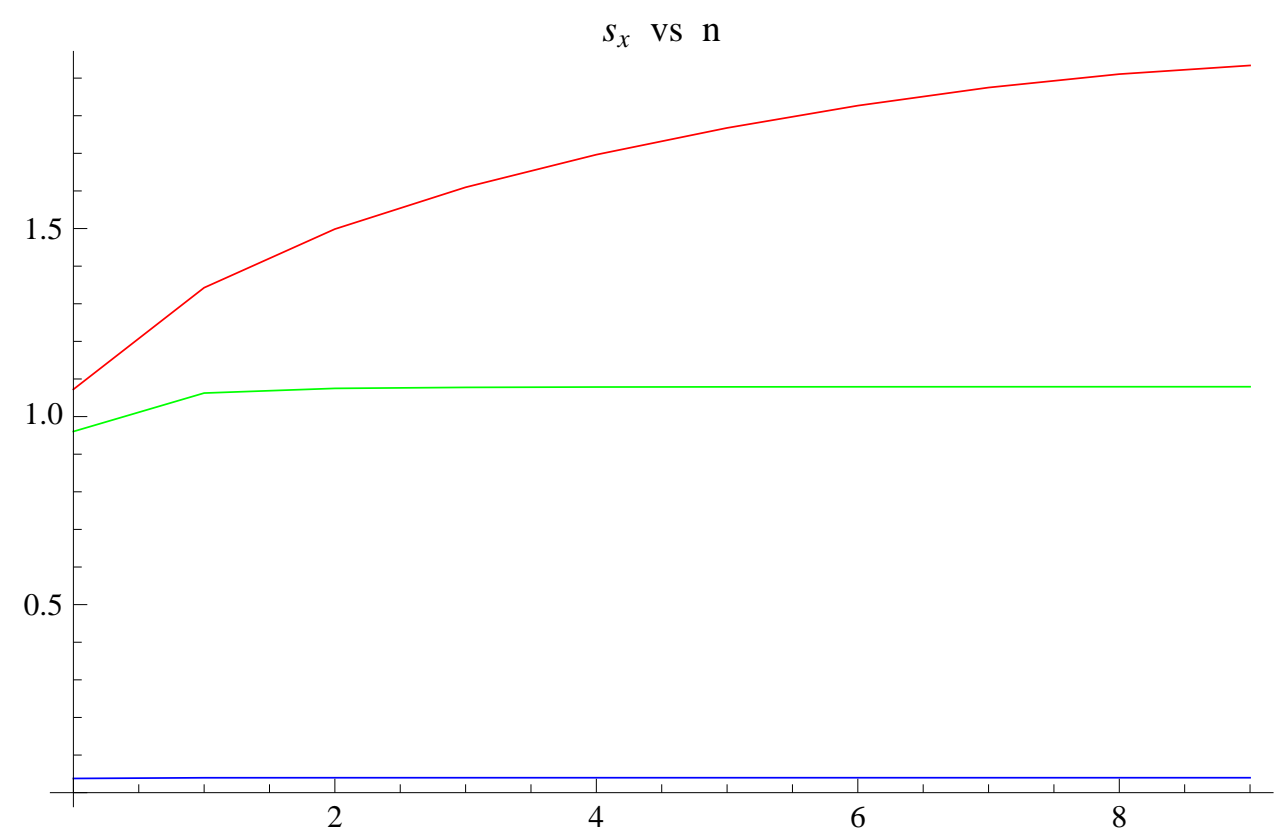

Figura 9.3: $s_{x}$ como función de $n$ para diferentes valores de $a . \quad a=1 / \sqrt{2}$ (azul), $a=2$ (verde), $a=5$ (rojo).

\subsubsection{Espacio de momento}

En la Fig. (9.4) se muestra $\Delta p$ como función del tamaño de la caja y se observa que la partícula se localiza en el espacio de momento. Considerando el comportamiento de $\Delta x$ se puede atribuir esta localización a la relación de incertidumbre entre ambas.

Por otra parte, puede observarse en la Fig. (9.5) que $s_{p}$ depende en $n$ para todo el intervalo de valores de $a$. También ocurre que $s_{p_{n}}^{H O}(\omega=1)<s_{p_{n}}^{C H O}<s_{p_{n+1}}^{P I A B}(a=1)$ para todos los casos, demostrando la capacidad de ambas entropías para detectar la transición de un modelo a otro. Debe resaltarse también que el estado basal del modelo PIAB es $n=1$, en tanto que para el HO es $n=0$, y así sucesivamente en estados excitados, lo que explica el $n+1$ como regla general en el ordenamiento mencionado. Para el HO ocurre que para $\omega=1, s_{x}=s_{p}$, lo que ocurre en el CHO para valores mayores de $a$. 


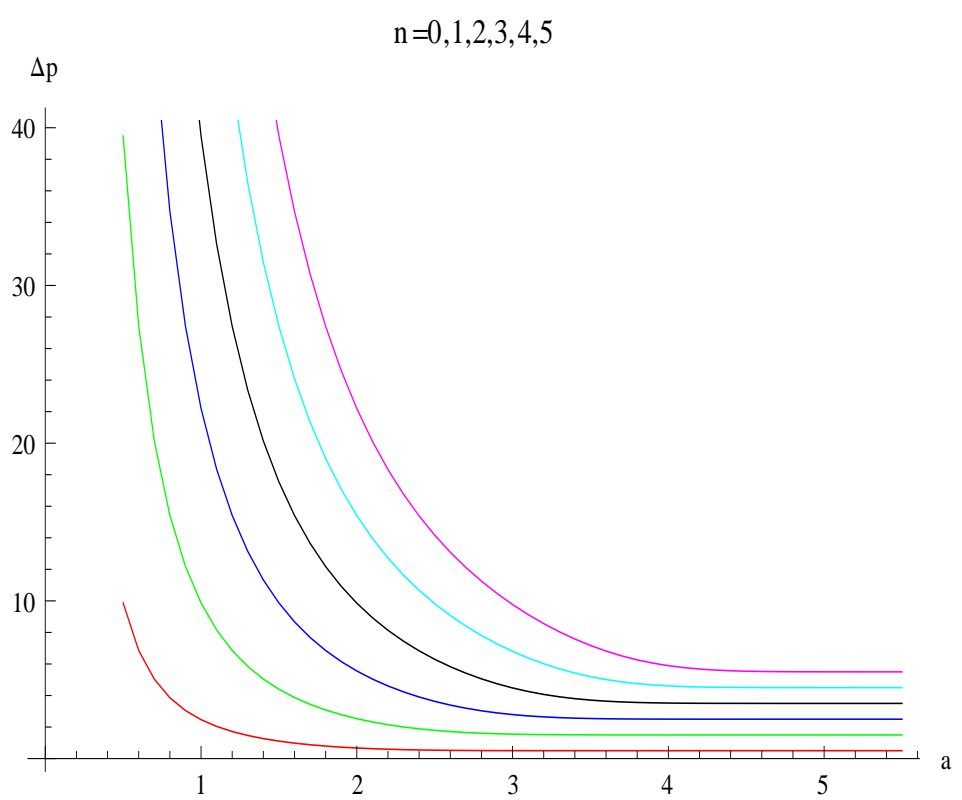

Figura 9.4: $\Delta p$ como función de $a$ para diferentes valores de $n, n=0$ es rojo y los otros valores son ascendentes conforme $n$ crece.

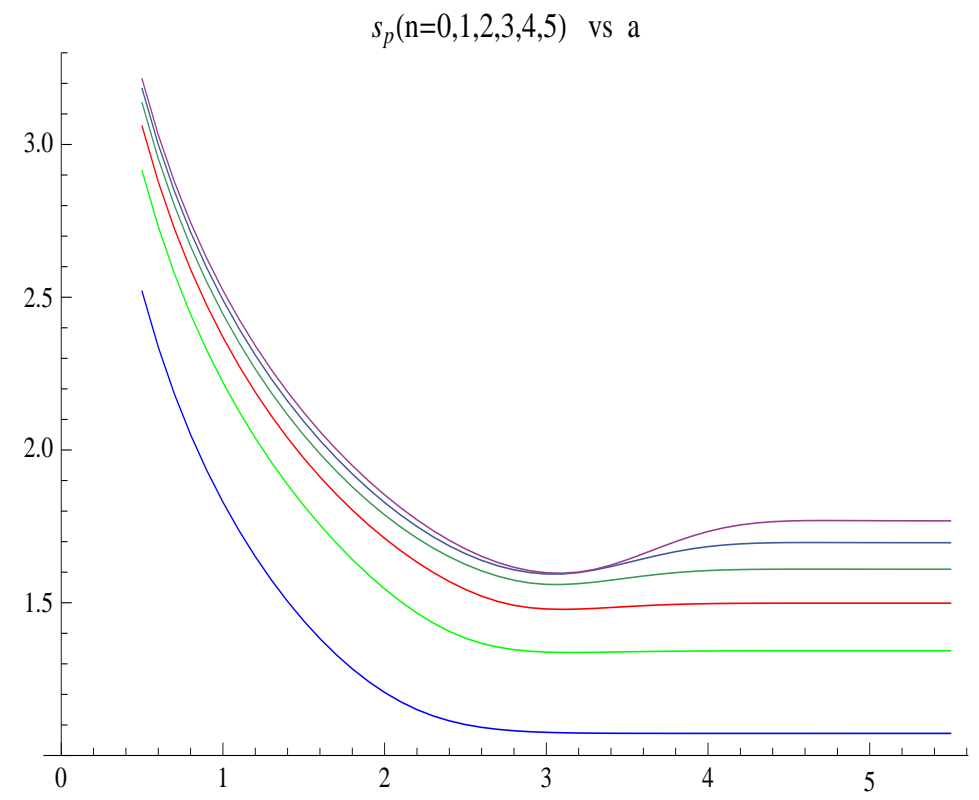

Figura 9.5: $s_{p}$ como función de a para diferentes números cuánticos $n=0$ es azul y los otros valores son ascendentes conforme $n$ crece. 
Debe notarse que en todas las curvas, excepto para $n=0$, existe un mínimo en $s_{p}$ para $a \approx 3$, este comportamiento resulta interesante porque si empezamos a reducir el tamaño de la caja, existen regiones de $a$ (antes del mínimo yendo de a grande a pequeña) en que ambas distribuciones se localizan, aunque su suma siempre respeta la cota de incertidumbre. También el mínimo juega un papel importante para poder conseguir $s_{x}=s_{p}$ para a mayores.

En la Fig. (9.6) se muestran las densidades de momento del CHO entre los dos límites del modelo, PIAB y HO. La escala en el eje de la densidad y en el eje de las $p$ 's es el mismo por lo que las gráficas son comparables entre sí directamente. De manera similar al caso de las densidades de posición, la comparación de los dos primeros renglones mostrarían el efecto del potencial, se observa que predominan las paredes; y los dos últimos mostrarían el efecto de las paredes, se advierte que predomina el potencial, $\omega$.

El análisis de $\pi(p)$ se puede relacionar con el mínimo de $s_{p}$ en $a \approx 3$. Atendiendo al tamaño de los picos de la distribución se puede notar que conforme nos desplazamos hacia abajo en las columnas ocurre una localización (crece la probabilidad de encontrar a las partículas con el valor del momento en los picos). En el caso $a=3$, el cuarto renglón, los picos son mayores que en el caso $a=5$, el quinto renglón, demostrando una deslocalización que efectivamente se observa en la Fig. (9.5).

Por otro lado, $s_{p}$ demuestra un comportamiento consistente con los comportamientos en PIAB y HO como función del número cuántico, como se observa en la Fig. (9.7). Se puede notar un cruce en $s_{p}$ para las curvas correspondientes a $a=2$ y $a=5$, en $n=7$. De manera que en $n=8$ ya es más deslocalizada la curva correspondiente a $a=5$.

En la Fig. (9.6), desplazándose en un renglón de izquierda a derecha, se observa la variación de la densidad con el número cuántico para un a dada. 

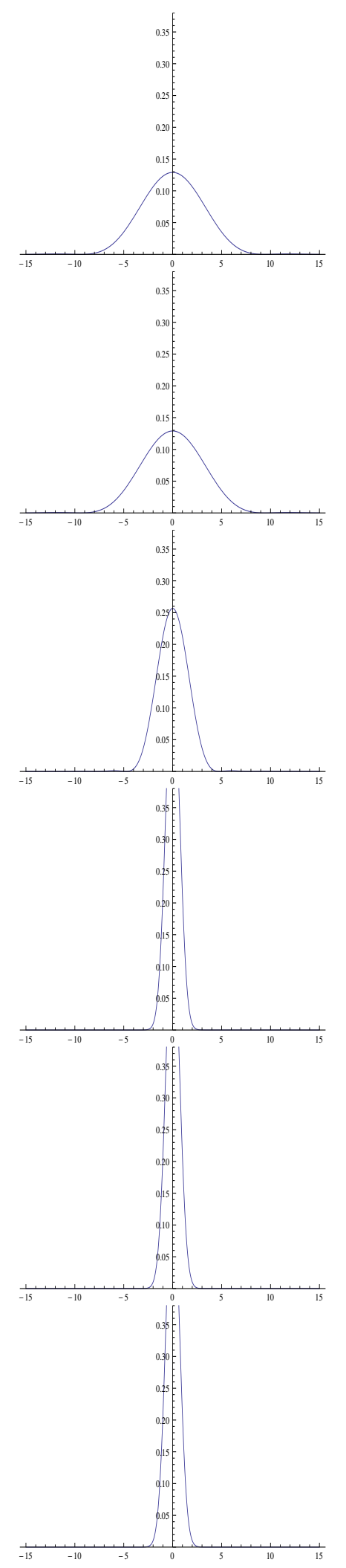
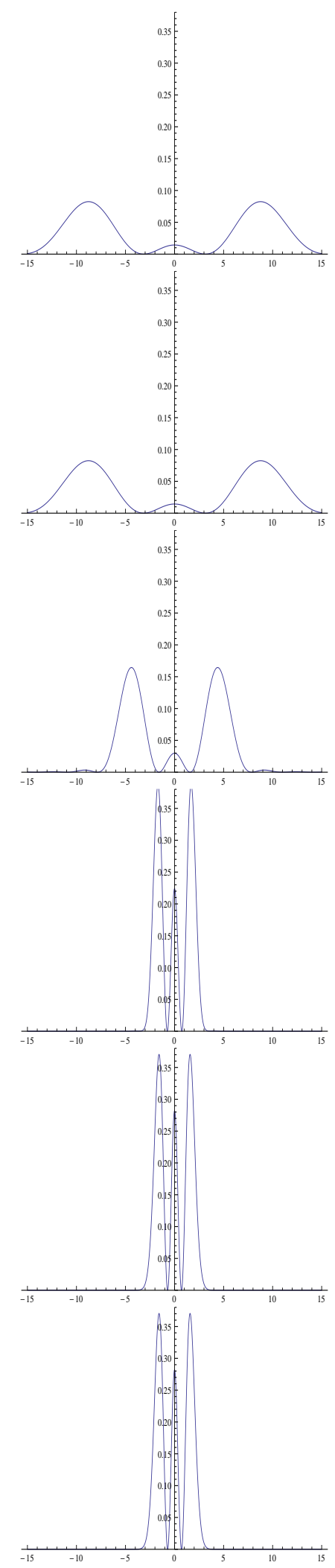

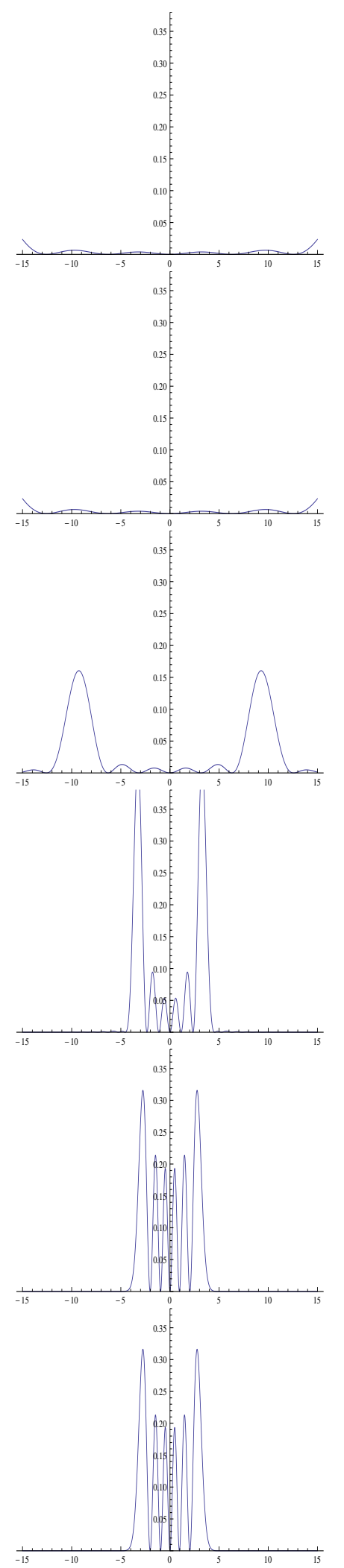

Figura 9.6: $\pi(p)$. De izquierda a derecha, $n=0, n=2, n=5$; de arriba hacia abajo: PIAB, $a=0.5, a=1, a=3, a=5$, HO. 


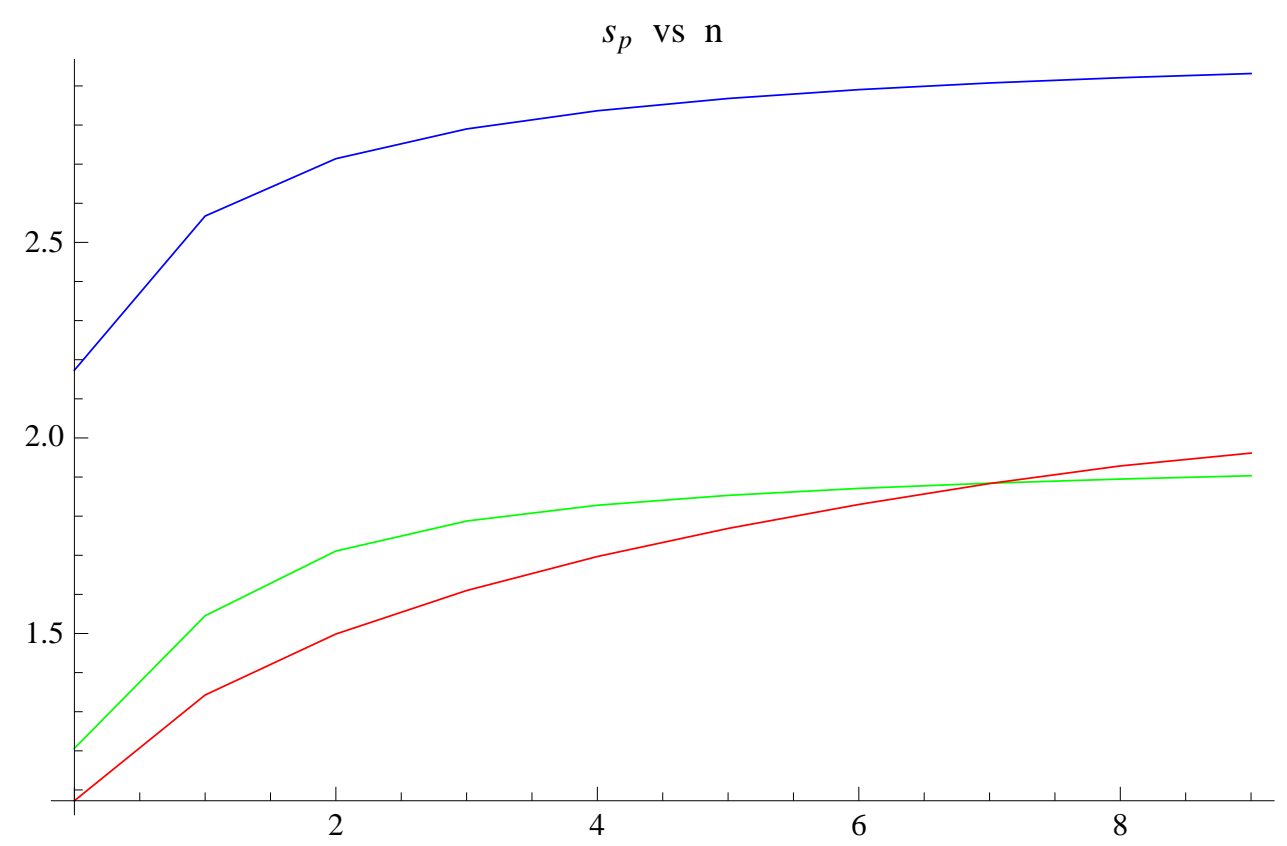

Figura 9.7: $s_{p}$ como función de $n$ para diferentes valores de $a . a=1 / \sqrt{2}$ (azul), $a=2$ (verde), $a=5$ (rojo).

\subsubsection{Relaciones de incertidumbre}

Graficamos primero $\Delta x \Delta p$ en la Fig. (9.8). Los valores van desde los valores de PIAB a los del HO. Para $n=2,3,4,5$ hay un máximo, reflejando un comportamiento de localización/deslocalización que no se observa en las desviaciones estándar individuales. También observamos que para $n=0,1,2,3$ el valor del producto correspondiente a PIAB es más pequeño que el correspondiente a $\mathrm{HO}$, mientras que para $n=4,5$ ocurre la situación opuesta. Para $n=0,1$ el producto disminuye con $a$, en tanto que para $n=2,3,4,5$ el producto primero aumenta y después disminuye. 

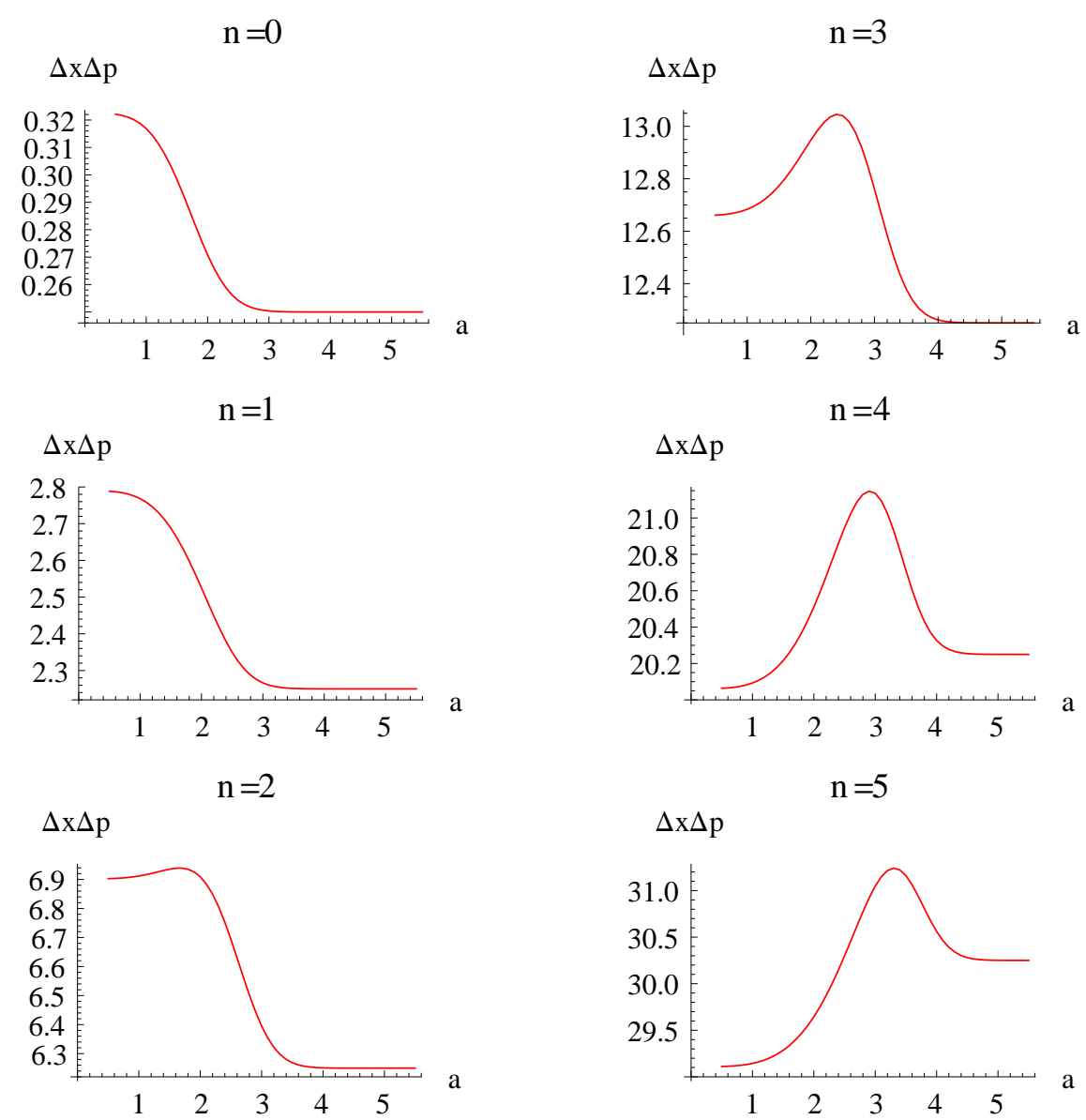

Figura 9.8: $\Delta x \Delta p$, como función de $a$ para diferentes $n$.

Ahora dirigimos nuestra atención a la suma entrópica. Primero debemos mencionar que en todos los casos la cota se cumple. En la Fig. (9.10) se muestra que para $n=0$, $s_{t}$ disminuye con $a$, en tanto que para el resto de los valores de $n, s_{t}$ crece. A su vez, puede advertirse que en ambos límites, $a$ pequeña y grande, $s_{t}$ adquiere valores en el intervalo del modelo PIAB al HO. 


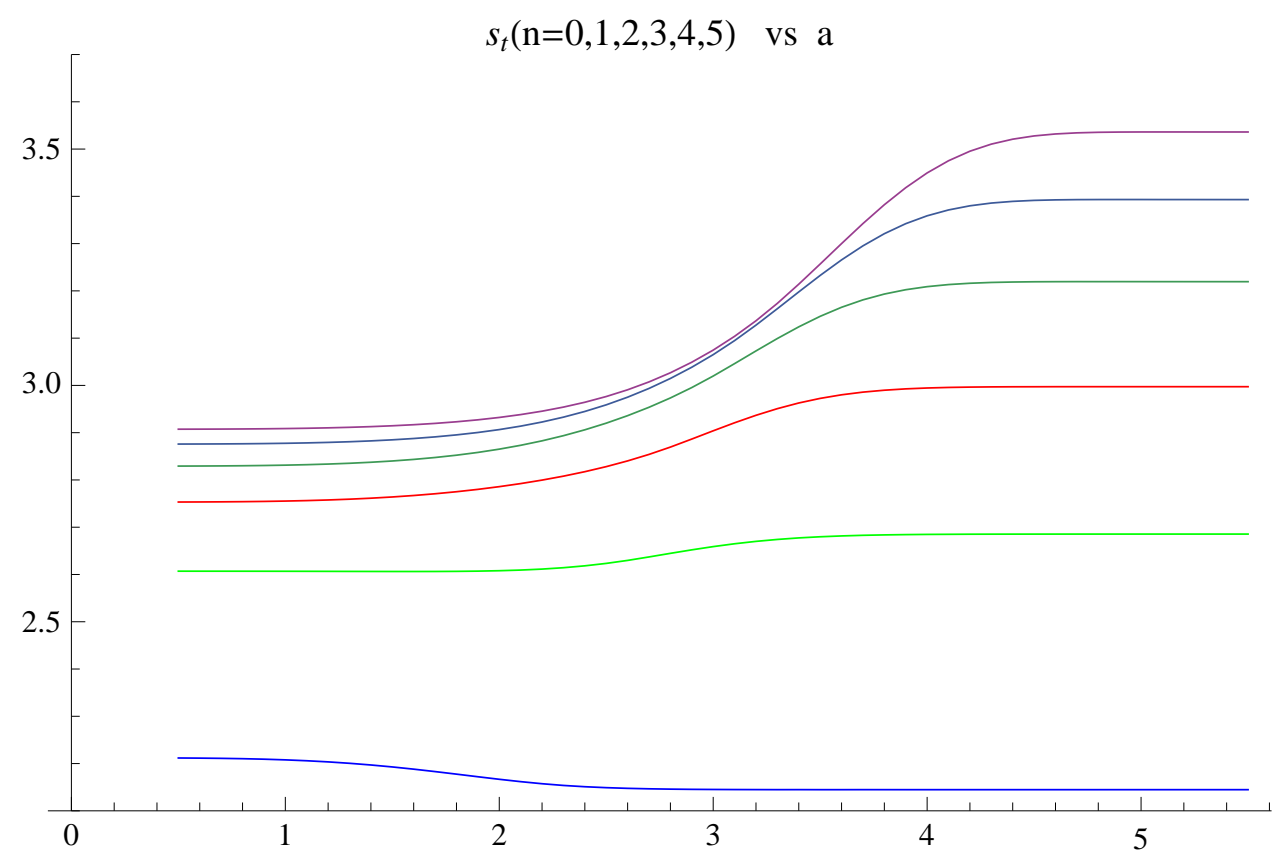

Figura 9.9: $s_{t}$ como función de $a . n=0$ es azul y aumenta con el número cuántico.
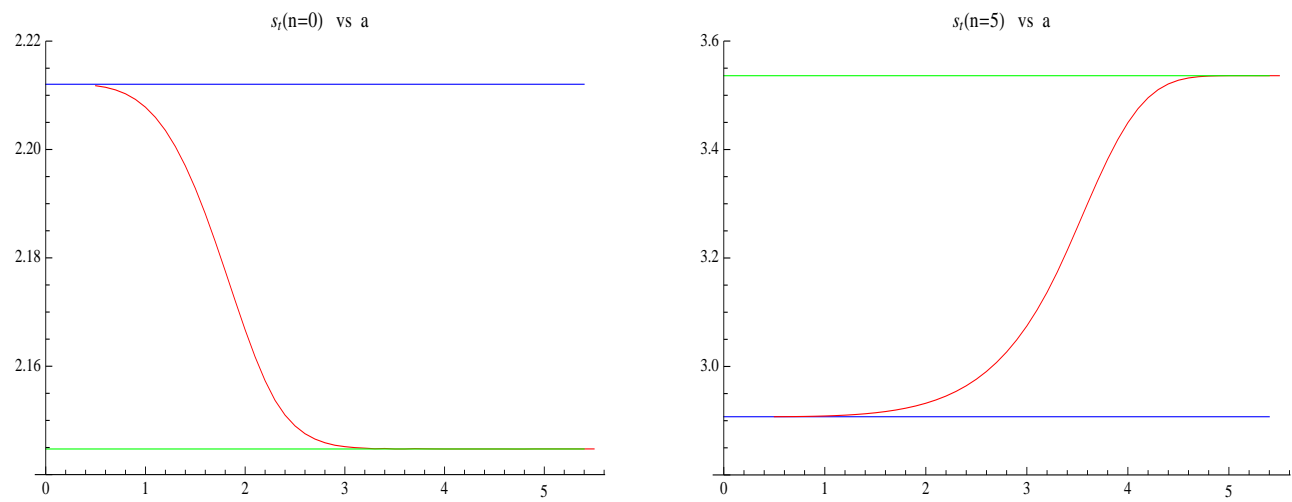

Figura 9.10: Izquierda. $s_{t}^{C H O}$ (rojo) como función de $a$ con $n=0$. Derecha. $s_{t}^{C H O}$ (rojo) como función de $a$ con $n=5$. Además se muestran $s_{t}^{P I A B}$ (azul) y $s_{t}^{H O}$ (verde).

En este comportamiento de $s_{t}$ el mínimo en $s_{p}$ tiene un papel relevante puesto que $n=0$ es el único valor para el que no existe ese mínimo y coincide con el estado en que la suma no es creciente. La importancia de tal mínimo puede observarse en la Fig. (9.11), donde hemos ejemplificado con $n=5$, que exhibe el comportamiento general. 
El mínimo parece ser un efecto combinado de la longitud de la caja y la frecuencia del oscilador confinado, lo que muestra su interrelación.

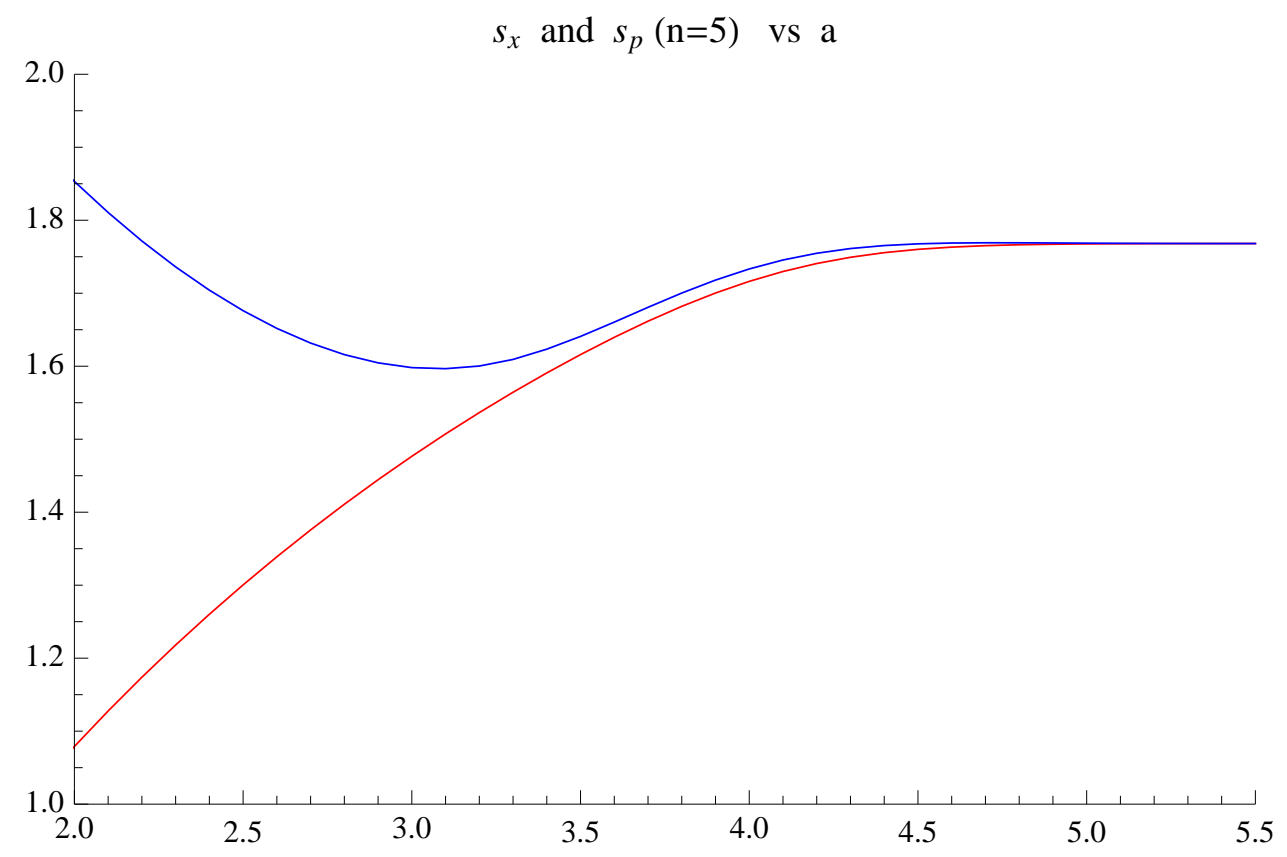

Figura 9.11: Detalles de $s_{x}$ (rojo) y $s_{p}$ (azul) como funciones de $a$ de donde se puede inferir el papel que juega el mínimo en el comportamiento.

Con $n, s_{t}$ tiene un comportamiento consistente con los modelos PIAB y HO, las curvas se muestran en la Fig. (9.12).

La Fig. (9.13) muestra las densidades separables en el espacio fase. Los ejes del valor de la función y el eje $p$ son consistentes para todas las curvas, sin embargo, el eje $x$ en cada caso corresponde a los límites de la caja y por lo tanto las gráficas no son directamente comparables. 


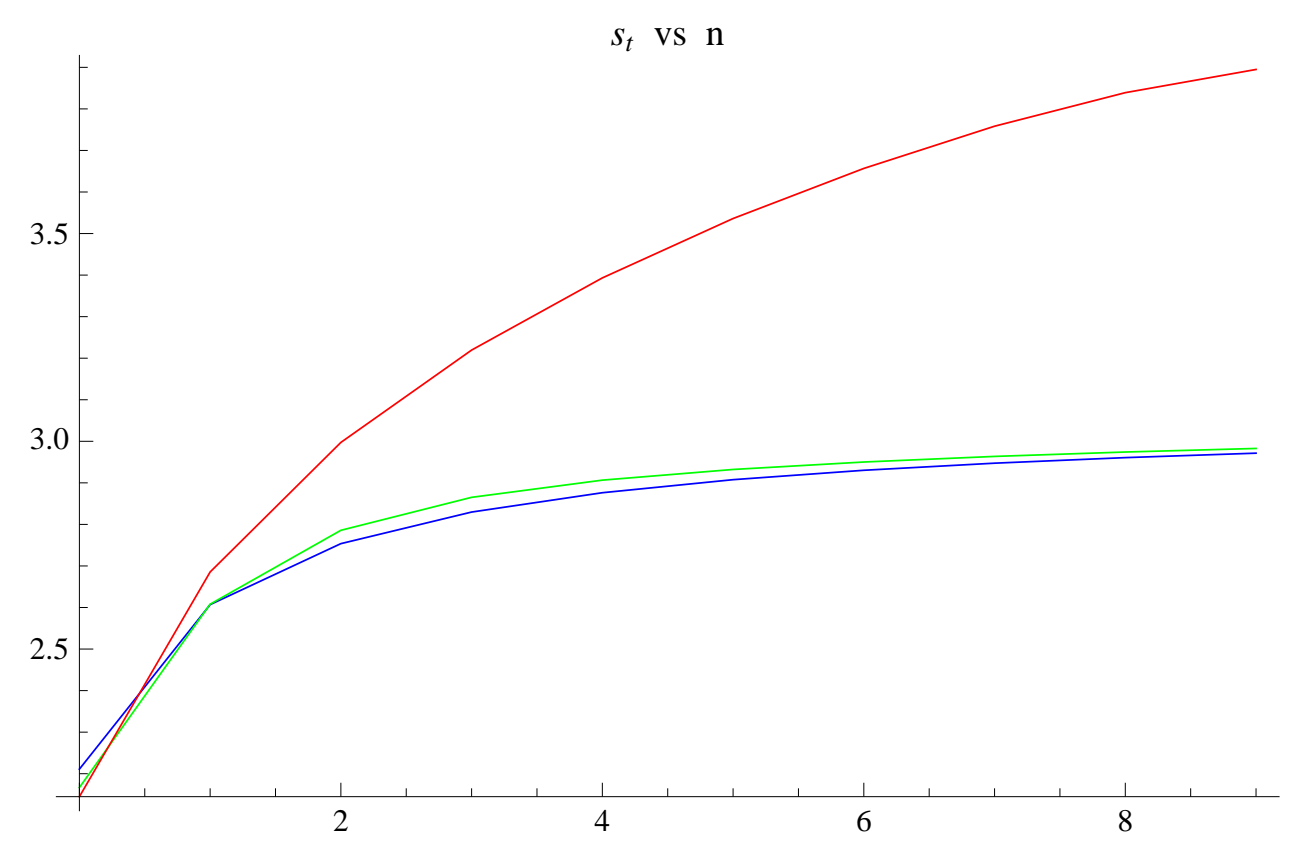

Figura 9.12: $s_{t}$ como función de $n$, con $a=1 / \sqrt{2}$ (azul), $a=2$ (verde), $a=5$ (rojo).

A pesar de este problema, haremos notar que hay una variación abrupta en el valor de $\rho(x) \pi(p)$ conforme crece el valor de $a$ para los $n$ impares. Para los valores de $a=3,5$, conforme $n$ aumenta se observa que las gráficas para los impares son muy diferentes que aquellas para los pares.

Según la suma entrópica, el caso $n=0$ se localiza en este espacio fase separable, en tanto que el resto se deslocaliza. Puede contrastarse este resultado con las gráficas. 

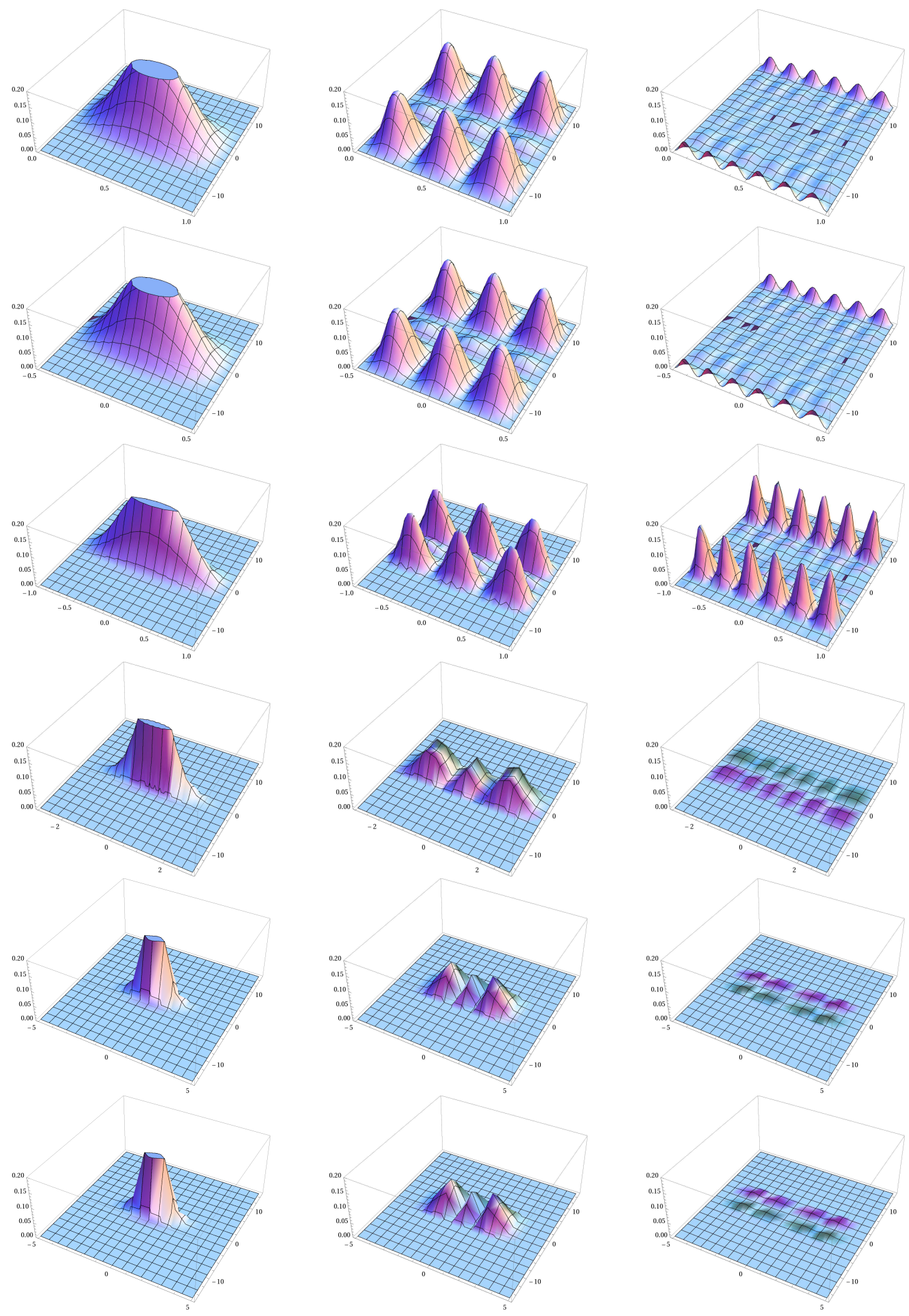

Figura 9.13: $\rho(x) \pi(p)$. De izquierda a derecha, $n=0, n=2, n=5$; de arriba hacia abajo: PIAB, $a=0.7, a=1, a=3, a=5$, HO. 


\subsubsection{Función de Wigner}

El componente real de la entropía de Shannon de la función de Wigner, $R e\left[s_{w}\right]$, aumenta con $a$ para todos los estados, como se observa en la Fig. (9.14) mostrando que la distribución se deslocaliza. Este comportamiento es consistente para $s_{t}$, con la excepción de $n=0$, donde la última disminuye. En contraste, para $\operatorname{Im}\left[s_{w}\right]$ ocurre que es decreciente para $n=0,1,2$, demostrando una disminución en el volumen de las regiones negativas conforme crece la caja, en tanto que para $n=3,4,5$ aumenta y luego disminuye por lo que existen máximos en esos estados.
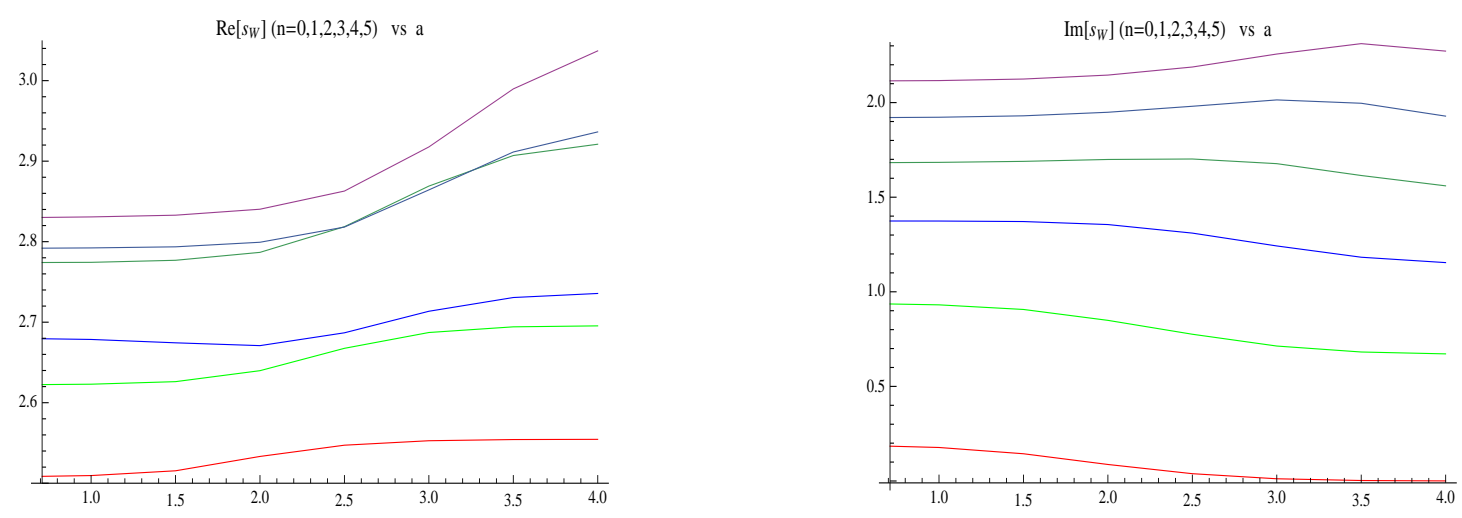

Figura 9.14: $\operatorname{Re}\left[s_{w}\right]$ (izquierda) y $\operatorname{Im}\left[s_{w}\right]$ (derecha) como funciones de $a$.

Por otro lado, en cuanto a los límites en que esperamos que varíen los valores, se muestran en la Fig. (9.15) las curvas correspondientes a $n=0,5$, y representa el comportamiento general, $\operatorname{Re}\left[s_{w}\right]$ parece variar en el intervalo de valores cuyo límite son los valores del modelo PIAB y del HO.

Este comportamiento contrasta con el de $\operatorname{Im}\left[s_{w}\right]$, mostrado en la Fig. (9.16), pues para $n=0$ es claro que los valores varían entre PIAB y HO, pero para $n=5$ el valor comienza en PIAB, pero aumenta por encima del valor de $\mathrm{HO}$ aunque parece que se aproxima para valores mayores de $a$, mostrando que el aumento de las regiones negativas no interpola estrictamente entre esos dos modelos. Resumiendo, si atendemos a las curvas para $\operatorname{Re}\left[s_{w}\right]$ y, más importante, para $\operatorname{Im}\left[s_{w}\right]$, el comportamiento de $n=0$ lo es también de $n=1,2$, en tanto que el comportamiento de $n=5$ lo es de $n=3,4$. 

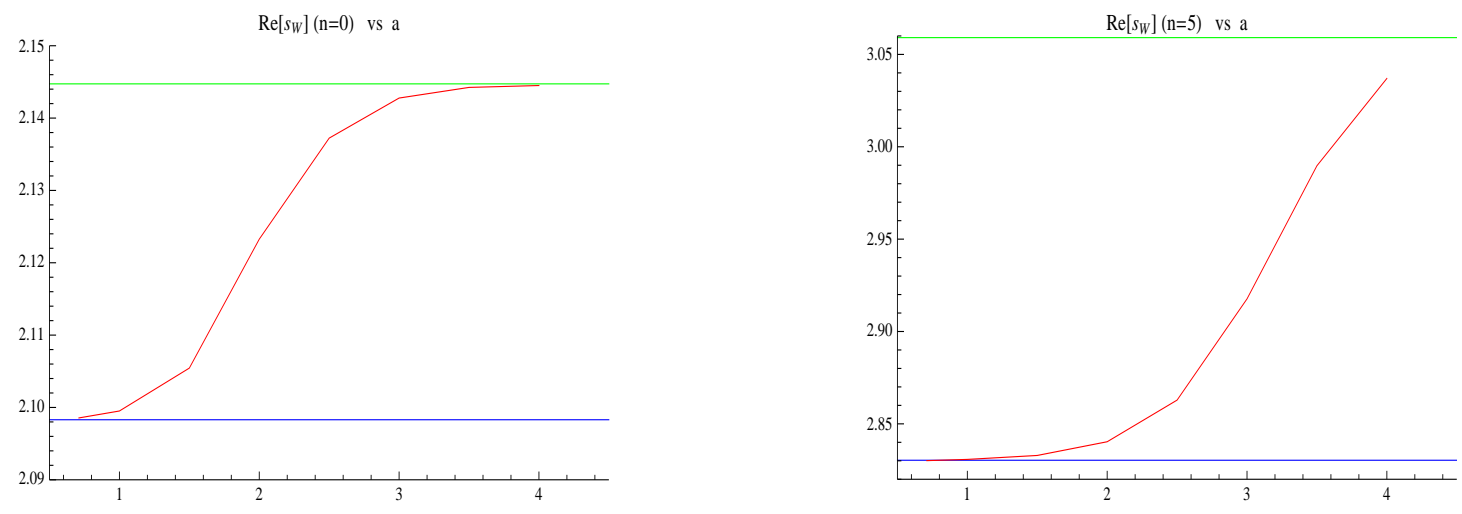

Figura 9.15: $\operatorname{Re}\left[s_{w}\right]$ para $n=0$ (izquierda) y $n=5$ (derecha) como funciones de $a$.
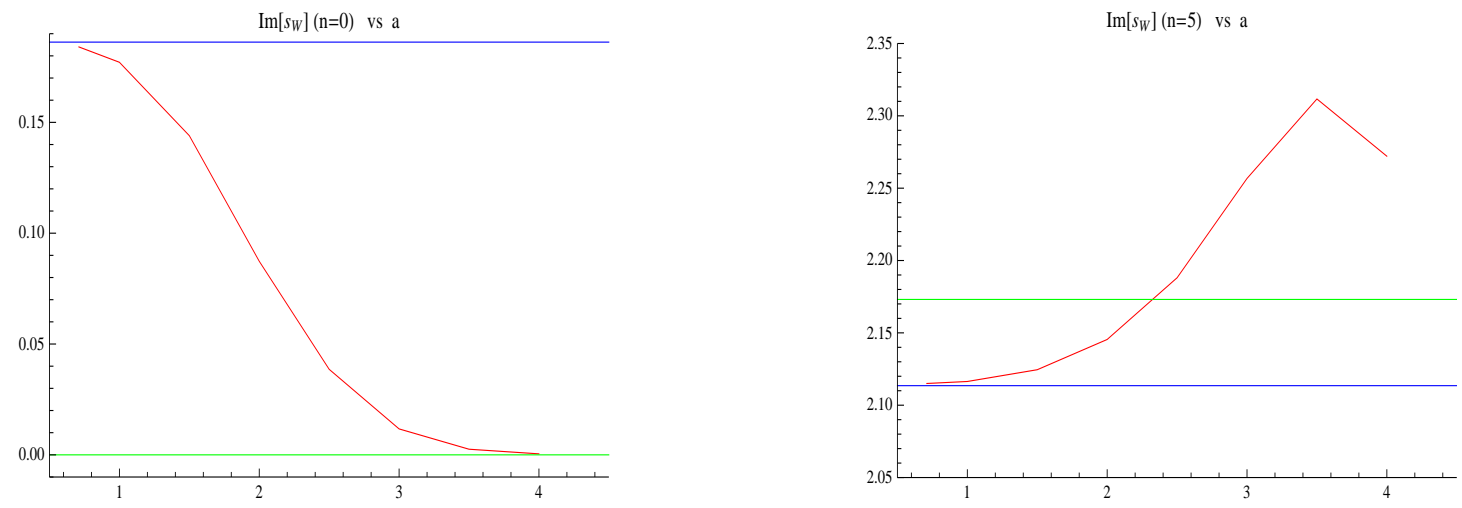

Figura 9.16: $\operatorname{Im}\left[s_{w}\right]$ para $n=0$ (izquierda) y $n=5$ (derecha) como funciones de $a$.

Con $n, \operatorname{Re}\left[s_{w}\right], \operatorname{Im}\left[s_{w}\right]$ y $\left|s_{w}\right|$ muestran un comportamiento consistente con los modelos PIAB y HO, las curvas se muestra en la Fig. (9.17). 


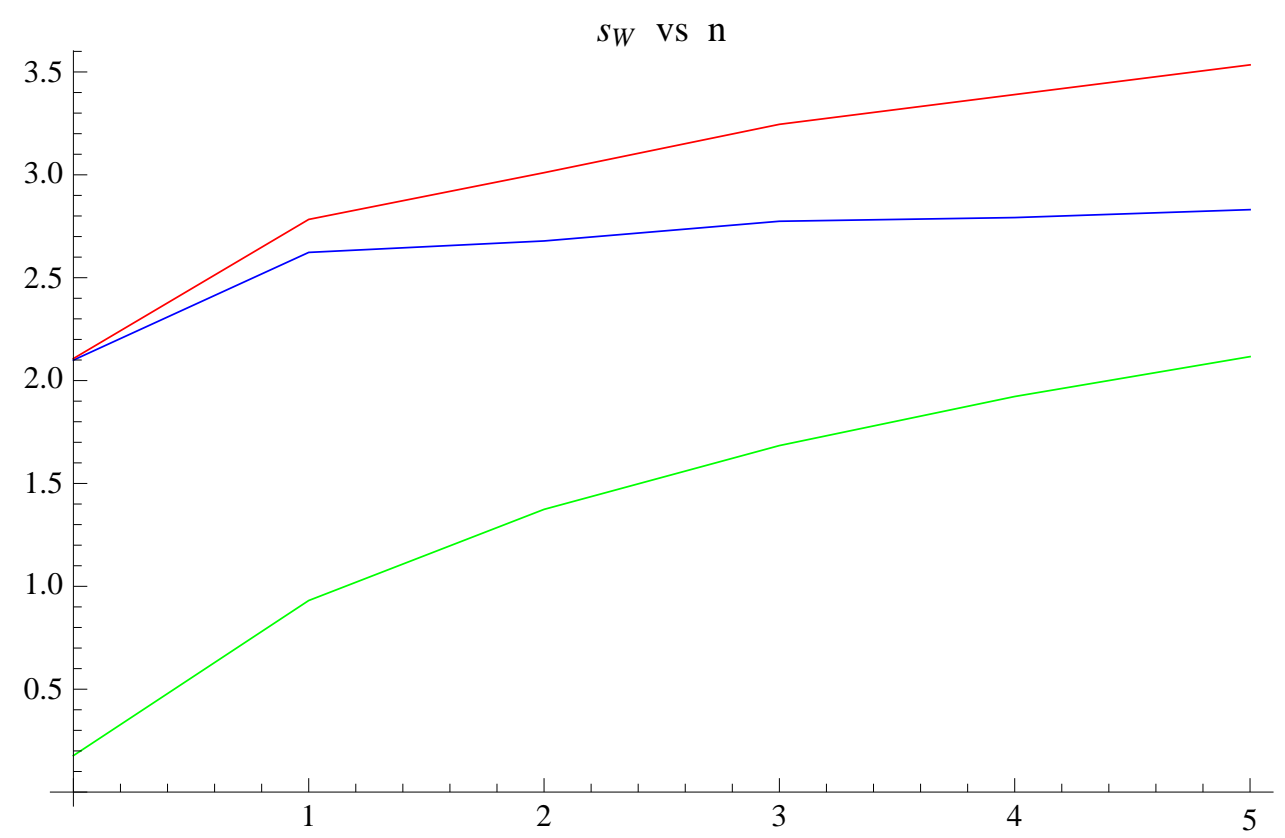

Figura 9.17: $\operatorname{Re}\left[s_{W}\right]$ (azul), $\operatorname{Im}\left[s_{W}\right]$ (verde) y $\left|s_{W}\right|$ (rojo).

Como en el caso de $\rho(x) \pi(p)$, las gráficas no son directamente comparables debido a la diferente escala en $x$. Resalta, sin embargo, el contraste en las dos distribuciones en espacio fase. La función de Wigner muestra más estructura que la distribución separable. La diferencia en la localización de las dos distribuciones es la correlación posición-momento, que abordaremos enseguida. 

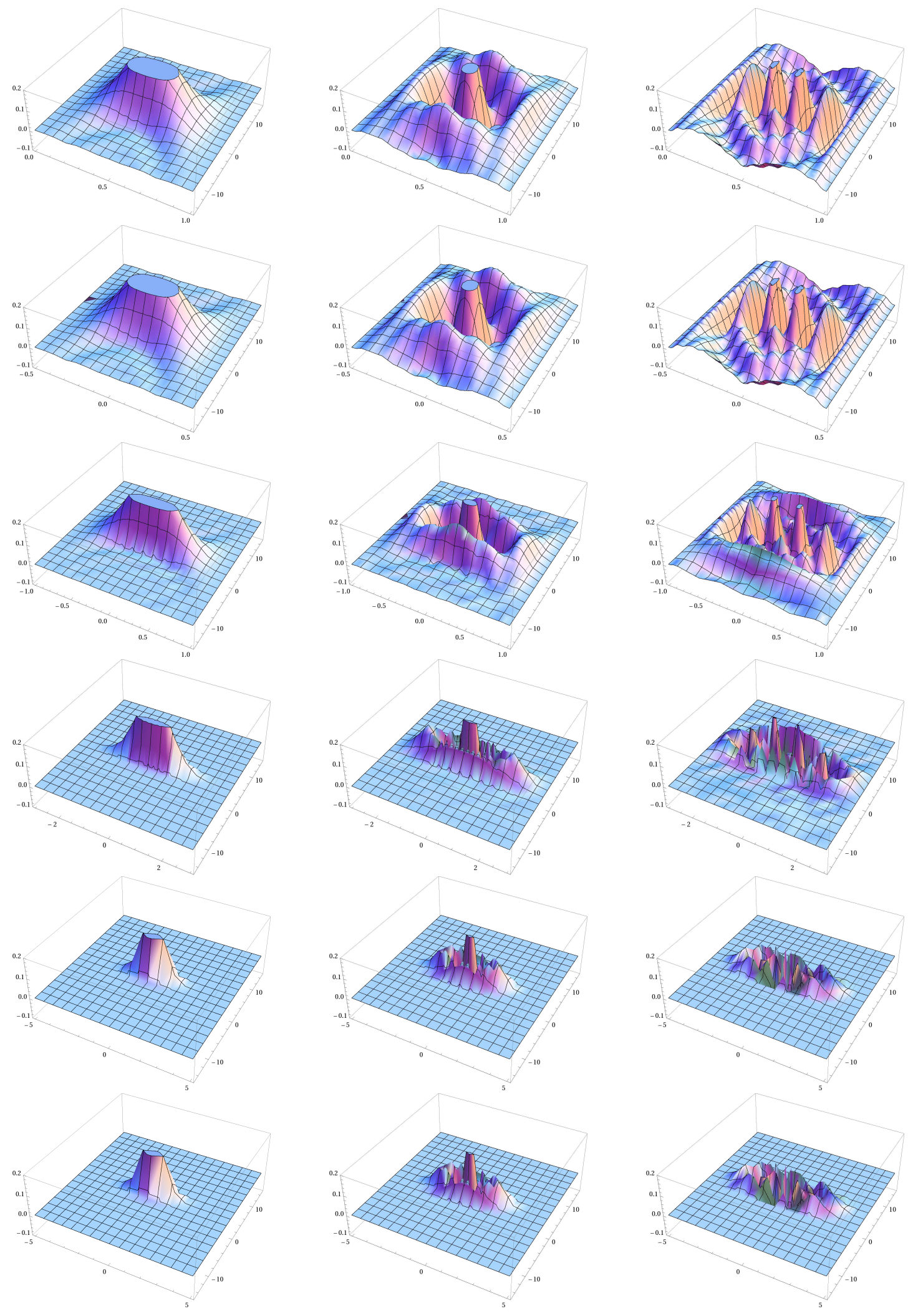

Figura 9.18: $W(x, p)$. De izquierda a derecha, $n=0, n=2, n=5$; de arriba hacia abajo: PIAB, $a=0.7, a=1, a=3, a=5$, HO. 


\subsection{Correlación posición-momento}

En la Fig. (9.19) se muestran las tres definiciones posibles para medir correlación. $R e\left[s_{t}-s_{w}\right]$ disminuye con el tamaño de la caja si el oscilador está en el estado basal, $n=0$, en tanto que $n=1$ tiene un mínimo (que es un máximo en su magnitud), y en todos los demás estados excitados aumenta, como ejemplo sólo se muestra la curva para $n=5$. Por otro lado, los valores de la curva $\operatorname{Re}\left[s_{t}-s_{w}\right]$ se encuentran en el intervalo entre PIAB y $\mathrm{HO}$.

La otra posibilidad, $\left|s_{t}\right|-\left|s_{w}\right|$, disminuye en magnitud en todos los estados, las particularidades son que si el oscilador está en el estado basal, $n=0$, los valores son positivos y la curva es decreciente, mientras que para los estados excitados los valores son negativos y la curva es creciente para aproximar a cero diminuyendo su magnitud, Fig. (9.19). Es importante señalar que para estas curvas los valores de CHO también se encuentran en el intervalo entre PIAB y HO.

Los valores de las curvas para $\left|s_{t}-s_{w}\right|$ del CHO no se encuentran en el intervalo de valores de PIAB a HO. La correlación se predice decreciente para los estados $n=0$ y $n=1$, Fig. (9.19),

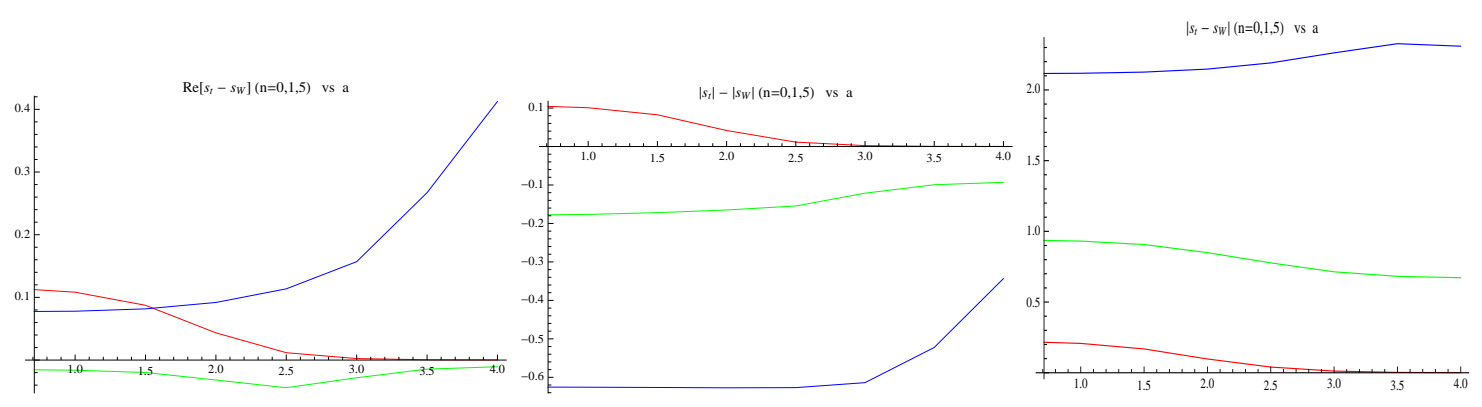

Figura 9.19: Tres posibilidades de $s_{t}-s_{w}$. En cada gráfica $n=0$ (rojo), $n=1$ (verde) y $n=5($ azul).

En su comportamiento con el número cuántico, $\operatorname{Re}\left[s_{t}-s_{W}\right],\left|s_{t}\right|-\left|s_{W}\right|$ y $\left|s_{t}-s_{W}\right|$ son consistentes con PIAB y HO. Re $\left[s_{t}-s_{W}\right]$ es la medida que predice la menor correlación $x-p$ y tiene un valor negativo para $n=1$. Por otro lado, $\left|s_{t}\right|-\left|s_{W}\right|$ tiene valores 
negativos para todos los estados, excepto para $n=0$, Fig. (9.20).

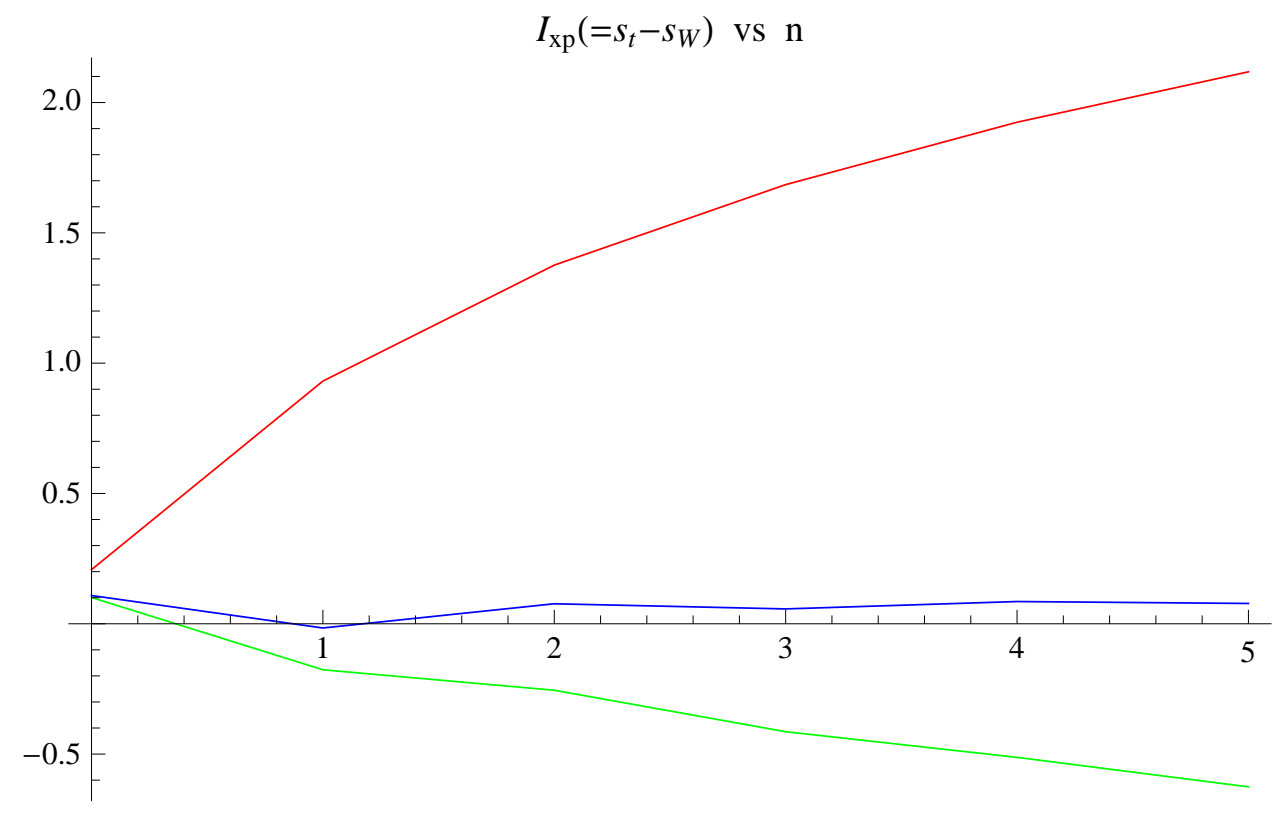

Figura 9.20: $\operatorname{Re}\left[s_{t}-s_{W}\right]$ (azul), $\left|s_{t}\right|-\left|s_{W}\right|$ (verde) y $\left|s_{t}-s_{W}\right|$ (rojo) como funciones de $n$.

\subsection{Resultados analíticos}

\subsubsection{Localización en los espacios de posición y momento}

La función hipergeométrica confluente se define por

$$
{ }_{1} F_{1}(a ; b ; z)=1+\frac{a}{b} z+\frac{a(a+1)}{b(b+1)} \frac{z^{2}}{2 !}+\ldots=\sum_{k=0}^{\infty} \frac{(a)_{k}}{(b)_{k}} \frac{z^{k}}{k !}
$$

donde $(a)_{k} \mathrm{y}(b)_{k}$ son símbolos de Pochhammer,

$$
(x)_{n} \equiv \frac{\Gamma(x+n)}{\Gamma(x)}=x(x+1) \ldots(x+n-1)
$$

Tomemos el caso par, expandamos la función de Kummer en Ec. (9.3) en series como la Ec. (9.6), 


$$
\begin{array}{r}
{ }_{1} F_{1}\left(\frac{1}{4}-\frac{E_{n}}{2}, \frac{1}{2}, x^{2}\right)=1+\left(\frac{1}{2}-E_{n}\right) x^{2}+\frac{1}{3 \cdot 2 !}\left(\frac{1}{2}-E_{n}\right)\left(\frac{5}{2}-E_{n}\right) x^{4} \\
+\frac{1}{5 \cdot 3 !}\left(\frac{1}{2}-E_{n}\right)\left(\frac{5}{2}-E_{n}\right)\left(\frac{9}{2}-E_{n}\right) x^{6} \\
+\frac{1}{7 \cdot 4 !}\left(\frac{1}{2}-E_{n}\right)\left(\frac{5}{2}-E_{n}\right)\left(\frac{9}{2}-E_{n}\right)\left(\frac{13}{2}-E_{n}\right) x^{8} \\
+\frac{1}{9 \cdot 5 !}\left(\frac{1}{2}-E_{n}\right)\left(\frac{5}{2}-E_{n}\right)\left(\frac{9}{2}-E_{n}\right)\left(\frac{13}{2}-E_{n}\right)\left(\frac{17}{2}-E_{n}\right) x^{10} \\
+\ldots
\end{array}
$$

Como puede observarse de la definiciión de los símbolos de Pochhammer, si $E=1 / 2$

$$
{ }_{1} F_{1}\left(\frac{1}{4}-\frac{E_{n}}{2}, \frac{1}{2}, x^{2}\right)=1
$$

porque todos los demás términos son cero. Si $E=5 / 2$, sólo sobreviven el primero y segundo términos,

$$
{ }_{1} F_{1}\left(\frac{1}{4}-\frac{E_{n}}{2}, \frac{1}{2}, x^{2}\right)=1+\left(\frac{1}{2}-E_{n}\right) x^{2}
$$

También se trunca la serie para $E=\frac{9}{2}, \frac{13}{2}, \frac{17}{2}, \frac{21}{2}, \frac{25}{2}, \ldots$, y en estos casos la transformada de Fourier y la de Weyl pueden hacerse de manera analítica. El número de raíces queda fijo, y por lo tanto el estado también. Algunas de estas funciones pueden observarse en la Tabla (9.1).

$x_{c}$ es el radio de confinamiento, y tenemos dos maneras de hacerlo variar en este esquema. La primera es fijando el número de nodos, cambiando cada vez $E_{n}$ y resolviendo para $x_{c}$,

$$
{ }_{1} F_{1}\left(\frac{1}{4}-\frac{E_{n}}{2}, \frac{1}{2}, x_{c}^{2}\right)=0, \quad{ }_{1} F_{1}\left(\frac{3}{4}-\frac{E_{n}}{2}, \frac{3}{2}, x_{c}^{2}\right)=0,
$$

La segunda es fijando $E_{n}$, cambiando el número de nodos $(n)$ y resolviendo las ecuaciones anteriores para $x_{c}$. Tomemos como ejemplo el caso par con $E_{n}=\frac{21}{2}$, en la Tabla (9.2) presentamos los valores de las entropías en los espacios de posición y momento y la suma entrópica. 


\begin{tabular}{|c|l|c|}
\hline$E_{n}$ & \multicolumn{1}{|c|}{$\psi(x)$} & \# Raíces, $\left|x_{c}\right|^{\prime}$ 's \\
\hline \hline$\frac{5}{2}$ & $e^{-x^{2} / 2}\left(1-2 x^{2}\right)$ & 1 \\
\hline$\frac{9}{2}$ & $e^{-x^{2} / 2}\left(1-4 x^{2}+\frac{4}{3} x^{4}\right)$ & 2 \\
\hline$\frac{13}{2}$ & $e^{-x^{2} / 2}\left(1-6 x^{2}+4 x^{4}-\frac{8}{15} x^{6}\right)$ & 3 \\
\hline$\frac{17}{2}$ & $e^{-x^{2} / 2}\left(1-8 x^{2}+8 x^{4}-\frac{32}{15} x^{6}+\frac{16}{105} x^{8}\right)$ & 4 \\
\hline$\frac{21}{2}$ & $e^{-x^{2} / 2}\left(1-10 x^{2}+\frac{40}{3} x^{4}-\frac{16}{3} x^{6}+\frac{16}{21} x^{8}-\frac{32}{945} x^{10}\right)$ & 5 \\
\hline
\end{tabular}

Tabla 9.1: Se muestran algunas funciones de onda cuyas transformadas son analíticas y las energías asociadas

\begin{tabular}{|c|c|c|c|c|}
\hline$x_{c}$ & \# Nodos & $s_{x}$ & $s_{p}$ & $s_{t}$ \\
\hline \hline 0.3429013 & 0 & -0.684 & 2.896 & 2.212 \\
\hline 1.0366108 & 2 & 0.422 & 2.334 & 2.756 \\
\hline 1.7566836 & 4 & 0.949 & 1.944 & 2.893 \\
\hline 2.5327317 & 6 & 1.314 & 1.676 & 2.990 \\
\hline 3.4361591 & 8 & 1.615 & 1.550 & 3.165 \\
\hline
\end{tabular}

Tabla 9.2: Valores de las entropías en los espacios de posición, $s_{\rho}$ y momento $s_{\pi}$ conforme cambia el radio de confinamiento, $x_{c}$ para $E_{n}=\frac{21}{2}$.

En la parte izquierda de la Fig. (9.21) se observa que la distribución se deslocaliza en el espacio de posición, se localiza en el espacio de momento y la suma entrópica crece. En este caso, mantenemos fija la energía, pero cambiamos el número de nodos, por lo que variamos el número cuántico.

Por otro lado, si fijamos nuestra atención en la variación de $x_{c}$ para el estado basal del sistema, parte derecha de la Fig. (9.21), podemos ver que la distribución en espacio de posición se deslocaliza, la distribución en espacio de momento se localiza y la suma entrópica parece no variar. En este caso, donde mantenemos fijo el número de nodos, estamos cambiando la frecuencia del oscilador (distancia entre nodos). La 
principal limitación de realizar estas transformaciones analíticas es que no podemos llegar más lejos de $a=\frac{1}{\sqrt{2}} \mathrm{y}$, de acuerdo con los resultados numéricos presentados, nos encontramos en el régimen en que el CHO tendría más características de PIAB que de $\mathrm{HO}$, lo cual es consistente con la no dependencia de $s_{t}$ en $a$.
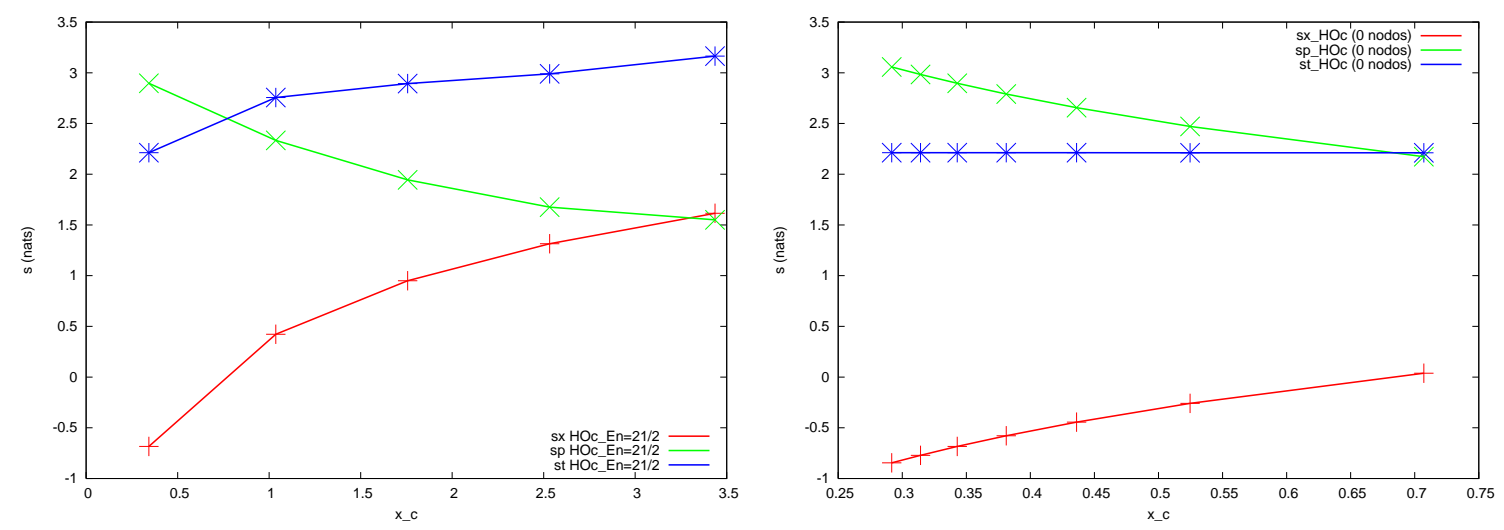

Figura 9.21: Izquierda. Gráfica de las entropías en la Tabla (9.2), con $E_{n}=\frac{21}{2}$. Derecha. Gráfica de las entropías del estado basal del CHO, variando $E_{n}$. Ambas curvas se presentan como función de $x_{c}$.

\subsubsection{Localización de la función de Wigner y correlación posición- momento}

Para el caso de $E_{n}=5 / 2$, mostramos en la Tabla (9.3) los resultados del cálculo de la entropía de Shannon de la función de Wigner, $s_{w}$, y ls entropía de $|\psi(x)|^{2}|\phi(p)|^{2}$, $s_{t}$, para el HO y para el CHO. En el caso del HO, $s_{w}$ no depende en $\omega$, igual que $s_{t}$. También se muestran los resultados de la correlación $x-p$, medida a través de la información mutua, $I_{x p}=s_{t}-s_{w}$. 


\begin{tabular}{|c|c|c|c|c|c|c|c|}
\hline$E_{n}=5 / 2$ & $s_{t}$ & $\operatorname{Re}\left[s_{w}\right]$ & $\operatorname{Im}\left[s_{w}\right]$ & $\left|s_{w}\right|$ & $\operatorname{Re}\left[s_{t}-s_{w}\right]$ & $\left|s_{t}\right|-\left|s_{w}\right|$ & $\left|s_{t}-s_{w}\right|$ \\
\hline \hline no confinado & 2.997 & 2.737 & 1.145 & 2.967 & 0.260 & 0.030 & 1.174 \\
\hline confinado & 2.211 & 2.099 & 0.180 & 2.106 & 0.112 & 0.105 & 0.212 \\
\hline
\end{tabular}

Tabla 9.3: Comparación entre el Oscilador Armónico Confinado y el Oscilador Armónico

En la Tabla (9.3) puede estudiarse el efecto del confinamiento en un oscilador armónico con una cierta energía, que bajo la restricción de tener paredes impenetrables mantiene su energía.

En estas condiciones, podemos afirmar que el confinamiento provoca que la función de Wigner se localice ya que las tres cantidades relacionadas con $s_{w}$ disminuyen. Además se localiza la distribución separable ( $s_{t}$ disminuye). Es importante señalar que el volumen de las regiones negativas de $W(x, p)$ (proporcional a $\operatorname{Im}\left[s_{w}\right]$ ) disminuye notablemente en el caso confinado.

En lo que se refiere a la correlación $x-p$, aumenta si usamos $\left|s_{t}\right|-\left|s_{w}\right|$ para medirla; y disminuye si usamos $R e\left[s_{t}-s_{w}\right]$ ó $\left|s_{t}-s_{w}\right|$ como medidas.

\subsection{Conclusiones}

En general las características de este modelo pueden racionalizarse concibiéndolo como un modelo intermedio entre el $\mathrm{HO}$ y PIAB.

$s_{x}^{C H O}$ para valores pequeños de $a$ se comporta como claramente como $s_{x}^{P I A B}$ (es independientes de $n$ ).

Los mínimos en $s_{p}^{C H O}$ juegan un papel importante en el modelo para que la suma entrópica, $s_{t}^{H O}$, alcance los límites correctos conforme crece el valor de $a$.

Es notable que una comparación de $s_{x}$ y $s_{p}$ puede hacerse con aquellas entropías para PIAB $(a=1)$ y para HO $(\omega=1)$. El mínimo en $s_{p}$ también juega un papel para que se cumpla que $s_{x}=s_{p}$ para $a$ grande; este el comportamiento de HO cuando $\omega=1$, independientemente del estado. 
La parte real de la entropía de Shannon, $R e\left[s_{W}\right]$, se comporta con a como esperamos en analogía con $s_{t}$, en tanto que la parte imaginaria, $\operatorname{Im}\left[s_{W}\right]$, refleja que el volumen de las regiones negativas no cambia con $a$ como se esperaría, demostrando una característica interesante. 


\section{Perspectivas para la ampliación de este trabajo}

Sería deseable extender el análisis de esta tesis a un estudio que permita explorar los problemas que supone trabajar en el espacio fase en relación con el principio de incertidumbre y la correlación posición-momento. De la misma manera, estudiar los efectos de más partículas, más dimensiones, la dependencia en el tiempo, etcétera. Todas estas posibilidades de análisis se pueden realizar en el átomo de Moshinsky.

En lo que concierne al desarrollo conceptual con (y de) herramientas de la teoría de la información, sería útil comparar la información mutua y el coeficiente de correlación con otras medidas de correlación posición-momento que ha sido estudiadas [113, 153], además explorar la definición de cantidades locales.

Finalmente, en un aspecto más general, es deseable contrastar los resultados con potenciales armónicos obtenidos en esta tesis con otros potenciales.

La lista de perspectivas es larga.

\subsection{Ampliación a otras funciones de distribución en espacio fase}

- Explorar la localización en otras distribuciones de espacio fase, particularmente la función de Husimi, positivo-definida, pero sin las marginales correctas. La función de Husimi puede relacionarse con un ordenamiento específico de los productos de 
operadores de posición y momento, además, su interpretación experimental es que se trata de un filtro gaussiano sobre la función de Wigner.

- Estudiar la correlación posición-momento en la función de Husimi. El análisis comparado con los resultados de esta tesis puede aportar datos sobre los efectos de un filtro gaussiano en tal correlación. Extender este estudio a la función de Husimi amplía las posibilidades de entender lo que sucede con la correlación posición-momento en el espacio fase. ¿Tendencialmente son similares o diferentes los comportamientos?

- Estudiar la relación de la localización de la función de Husimi con el principio de incertidumbre. Por ejemplo podría analizarse la distancia de Kullback-Leibler entre la distribución en espacio fase separable, formada por el producto de las marginales de la función de Husimi y el producto de las densidades de posición y momento.

\subsection{Ampliación a variantes del modelo}

- Es deseable ampliar el estudio a dos y tres dimensiones. El modelo $3 D$ es el que se ha comparado con el Helio.

- Este modelo sigue siendo soluble analíticamente para $N$ partículas. Es factible y deseable analizar la influencia de la tercera partícula en la localización y en la correlación posición-momento en la función de Wigner. Si elegimos conveniente las masas de un sistema de tres partículas, tenemos un modelo del enlace químico donde es posible estudiar los efectos de los diferentes tipos de correlación entre sí, y la localización de las diferentes distribuciones.

- Analizar la evolución temporal de la localización y de la correlación posiciónmomento en el modelo, por ejemplo en el contexto de los sistemas cuánticos abiertos. 


\subsection{Desarrollo de las herramientas de la teoría de la información}

- Existen otras propuestas para medir la correlación posición-momento en la función de Wigner. Su análisis en el átomo de Moshinsky (también en los modelos de una partícula, por supuesto) nos daría una posibilidad de comparación para la información mutua que estudiamos en esta tesis, así podríamos comparar la dependencia de las diferentes medidas con los diferentes parámetros del sistema.

- Estudiar las definiciones locales de la entropía de Shannon y de la información mutua entre partículas.

- Proponer y estudiar versiones locales de la entropía de Shannon de la función de Wigner y de la información mutua entre posición y momento. Las posibilidades de análisis local en el espacio fase son interesantes por las conexiones que hay con el principio de incertidumbre e incluso con principios de incertidumbre locales que también han sido estudiados.

\subsection{Ampliación a otros sistemas}

- Estudiar otros potenciales permitiría averiguar si los efectos estudiados son originados por el tipo de potencial o si se presentan en diferentes tipos de interacción entre partículas. Si queremos ampliar nuestros resultados analíticos, pueden estudiarse modelos analíticos donde coexisten potenciales de Coulomb y armónicos.

- Extender el estudio a sistemas atómicos. Existen algunos trabajos que calculan la función de Wigner del átomo de Hidrógeno y de algunos otros átomos de capa cerrada, nos interesa ampliar el estudio de esta tesis a sistemas atómicos y moleculares. 


\subsection{El átomo de Moshinsky acoplado con el ambi- ente}

En esta sección presentamos algunos avances del estudio realizado en sistemas abiertos utilizando las herramientas informacionales.

\subsubsection{La ecuación maestra de muchos cuerpos}

La evolución unitaria del operador de la densidad de un sistema en un baño térmico es descrita por la ecuación,

$$
\frac{d}{d t} \hat{\rho}(t)=-i[\hat{H}(t), \hat{\rho}(t)]
$$

donde,

$$
\hat{H}(t)=\hat{H}_{S}(t)+\hat{H}_{R}+\hat{V}
$$

y a su vez,

$$
\hat{H}_{S}=-\frac{1}{2} \sum_{i=1}^{N} \nabla_{i}^{2}+\sum_{i \neq j}^{N} V\left(x_{i}-x_{j}\right)+\sum_{i=1}^{N} v_{e x t}\left(x_{i}, t\right)
$$

es el Hamiltoniano del sistema de interés, $v_{\text {ext }}(x, t)$ potencial externo y $\hat{V}$ es el acoplamiento sistema-baño que frecuentemente puede tratarse con teoría de perturbaciones. $\hat{H}_{R}$, que es el Hamiltoniano del baño, tiene frecuentemente un espectro de eigenestados denso comparado con el del sistema. La densidad de estados de $\hat{H}_{R}$ gobierna la velocidad con que decaen las funciones de correlación del baño y por ello determina la dinámica reducida del sistema.

El operador de la densidad del sistema se obtiene tomando la traza sobre los grados de libertad del baño,

$$
\hat{\rho}_{S}(t)=\operatorname{Tr}_{R}\{\hat{\rho}(t)\}
$$

Así, podemos escribir la ecuación maestra formalmente exacta,

$$
\frac{d}{d t} \hat{\rho}_{S}(t)=-i\left[\hat{H}_{S}(t), \hat{\rho}_{S}(t)\right]+\int_{t_{0}}^{t} d \tau \Xi(t-\tau) \hat{\rho}_{S}(\tau)+\Psi(t)
$$


$\Xi(t-\tau)$ es el kernel de memoria y $\Psi(t)$ proviene de las correlaciones iniciales sistemabaño. En la práctica se requiere una aproximación para ambos términos.

Estamos interesados en la densidad,

$$
n(r, t)=\operatorname{Tr}_{S}\left\{\hat{\rho}_{S}(t) \hat{n}(r)\right\}
$$

donde $\hat{n}(r)=\sum_{i}^{N} \delta\left(r-\hat{r}_{i}\right)$ es el operador de la densidad de número. La ecuación de continuidad que describe la evolución de la densidad en el tiempo para OQS ${ }^{1}$ es,

$$
\begin{aligned}
\frac{\partial}{\partial t} n(r, t)= & -\nabla \cdot \operatorname{Tr}_{S}\left\{\hat{\rho}_{S}(t) \hat{j}(r)\right\} \\
& +\operatorname{Tr}\left\{\hat{n}(r)\left(\int_{t_{0}}^{t} d \tau \Xi(t-\tau) \hat{\rho}_{S}(\tau)+\Psi(t)\right)\right\} .
\end{aligned}
$$

El primer término es la "corriente Hamiltoniana" que aparece también en sistemas cerrados. El segundo término proviene de la parte no-unitaria de la evolución lo que define una "corriente disipativa" [154, 155],

$$
-\nabla \cdot j_{\text {disp }}(r, t)=\operatorname{Tr}\left\{\hat{n}(r)\left(\int_{t_{0}}^{t} d \tau \Xi(t-\tau) \hat{\rho}_{S}(\tau)+\Psi(t)\right)\right\} .
$$

\subsubsection{Aproximación de Markov y la ecuación maestra de Lindblad}

Es frecuente despreciar las correlaciones iniciales, $\Psi(t)=0$ y utilizar la aproximación de Markov donde el kernel de memoria es local en el tiempo, es decir,

$$
\int_{t_{0}}^{t} d \tau \Xi(t-\tau) \hat{\rho}_{S}(\tau)=\breve{D} \hat{\rho}_{S}(t)
$$

La aproximación de Markov es válida cuando $\tau_{S} \gg \tau_{B}$. $\tau_{S}$ es la escala de tiempo en que el sistema alcanza el equilibrio y $\tau_{B}$ es el tiempo de correlación más grande en el baño. Si las correlacinoes internas del baño decaen antes de que el sistema haya tenido tiempo de evolucionar podemos despreciar la memoria, esta condición implica una correlación sistema-baño débil.

\footnotetext{
${ }^{1}$ OQS: Open Quantum Systems, Sistemas Cuánticos Abiertos.
} 
En el contexto de la aproximación markoviana podemos escribir la ecuación maestra de diferentes maneras. Una forma ampliamente utilizada debido a que garantiza la positividad de la matriz de la densidad es la de Lindblad, en la cual

$$
\breve{D} \hat{\rho}_{S}(t)=\sum_{m n}\left\{L_{m n} \hat{\rho}_{S}(t) L_{m n}^{\dagger}-\frac{1}{2} L_{m n}^{\dagger} L_{m n} \hat{\rho}_{S}(t)-\frac{1}{2} \hat{\rho}_{S}(t) L_{m n}^{\dagger} L_{m n}\right\}
$$

\subsubsection{El átomo de Moshinsky, un modelo soluble analítico}

Estamos interesados en las interacciones repulsivas, tal como ocurren entre electrones, en ese régimen de interacción el Hamiltoniano del átomo de Moshinsky en $1 D$ se escribe,

$$
\hat{H}=\frac{\hat{p}_{1}^{2}}{2 m}+\frac{\hat{p}_{2}^{2}}{2 m}+\frac{1}{2} \omega^{2} m \hat{x}_{1}^{2}+\frac{1}{2} \omega^{2} m \hat{x}_{2}^{2}-\frac{1}{2} \lambda^{2} m\left(\hat{x}_{1}-\hat{x}_{2}\right)^{2}
$$

La ecuación de Lindblad de dos partículas,

$$
\begin{aligned}
\frac{d}{d t} \hat{\rho}_{S}(t)= & -i\left[\hat{H}_{S}(t), \hat{\rho}_{S}(t)\right] \\
& +\sum_{m n}\left\{L_{m n} \hat{\rho}_{S}(t) L_{m n}^{\dagger}-\frac{1}{2} L_{m n}^{\dagger} L_{m n} \hat{\rho}_{S}(t)-\frac{1}{2} \hat{\rho}_{S}(t) L_{m n}^{\dagger} L_{m n}\right\}
\end{aligned}
$$

puede resolverse de manera analítica para obtener $\hat{\rho}_{S}(t)$.

Para la dinámica asumiremos que el estado inicial es una superposición del estado basal y el primer estado excitado del átomo de Moshinsky,

$$
|\psi(0)\rangle=\frac{1}{\sqrt{2}}(|00\rangle+|10\rangle)
$$

ambos estados tienen la misma simetría, son simétricos bajo el intercambio de las coordenadas originales. Esta elección es equivalente a considerar las condiciones iniciales: $\rho_{00,00}(0)=\rho_{00,10}(0)=\rho_{10,00}(0)=\rho_{10,10}(0)=\frac{1}{2}$.

Podemos calcular la densidad de pares por medio de

$$
n\left(x_{1}, x_{2}, t\right)=\left\langle x_{1} x_{2}\left|\hat{\rho}_{S}(t)\right| x_{1} x_{2}\right\rangle
$$

Y también podemos obtener la densidad reducida por integración,

$$
n(x, t)=\int d x_{2} n\left(x, x_{2}, t\right)
$$


Es conveniente considerar dos casos límite de la ecuación maestra para resolver analíticamente la Ec. (10.13). El caso de desfasamiento puro sin relajación y el caso de relajación sin desfasamiento puro.

\section{Desfasamiento puro sin relajación}

Consideraremos primero el caso en que la ecuación maestra de Lindblad induce desfasamiento puro en el sistema y no induce relajación. En este caso, los operadores de Lindblad de dos cuerpos son diagonales en los eigenestados del átomo de Moshinsky,

$$
L_{m n, m^{\prime} n^{\prime}}=\delta_{m n, m^{\prime} n^{\prime}} \sqrt{\frac{\gamma_{m n}}{2}}|m n\rangle\langle m n|
$$

donde $|m n\rangle$ es el eigenestado del átomo de Moshinsky, $m$ es el número cuántico del centro de masa y $n$ el de las coordenadas relativas. Como los operadores $L_{m n, m^{\prime} n^{\prime}}$ son diagonales, las poblaciones de los diferentes niveles no cambia conforme el sistema evoluciona. Esto implica que la energía se conserva porque no puede haber intercambio con el ambiente. Las coherencias decaen de forma exponencial.

El desfasamiento puro describe la situación en que las colisiones entre el sistema y el baño son elásticas, de manera que el baño induce decoherencia en el sistema sin intercambiar energía.

Utilizando estos operadores de Lindblad en la Ec. (10.13) y el estado inicial obtenemos para las poblaciones,

$$
\rho_{00,00}(t)=\rho_{00,00}(0)=\frac{1}{2}
$$

y

$$
\rho_{10,10}(t)=\rho_{10,10}(0)=\frac{1}{2}
$$

son constantes porque no hay relajación en el sistema. Por otra parte, para las coherencias obtenemos,

$$
\rho_{00,10}(t)=\rho_{00,10}(0) e^{-i\left(E_{00}-E_{10}\right) t} e^{-\frac{1}{2}\left(\frac{\gamma_{00}+\gamma_{10}}{2}\right) t}=\frac{1}{2} e^{i \omega t} e^{-\frac{1}{2}\left(\frac{\gamma_{00}+\gamma_{10}}{2}\right) t}
$$

y

$$
\rho_{10,00}(t)=\rho_{10,00}(0) e^{-i\left(E_{10}-E_{00}\right) t} e^{-\frac{1}{2}\left(\frac{\gamma_{00}+\gamma_{10}}{2}\right) t}=\frac{1}{2} e^{-i \omega t} e^{-\frac{1}{2}\left(\frac{\gamma_{00}+\gamma_{10}}{2}\right) t}
$$


Las coherencias oscilan y decaen exponencialmente debido a la presencia del baño.

\section{Relajación sin desfasamiento puro}

En este caso los operadores de Lindblad son estrictamente no diagonales,

$$
\begin{aligned}
& L_{m n, m^{\prime} n^{\prime}}=\sqrt{\gamma_{m n, m^{\prime} n^{\prime}}}|m n\rangle\left\langle m^{\prime} n^{\prime}\right| \text { si } \quad m n \neq n^{\prime} n^{\prime} \\
& L_{m n, m^{\prime} n^{\prime}}=\quad 0 \quad \text { si } m n=m^{\prime} n^{\prime}
\end{aligned}
$$

Para asegurar que las poblaciones obedecen el balance detallado en el equilibrio debe cumplirse que,

$$
\gamma_{m n, m^{\prime} n^{\prime}}=e^{\beta \omega_{m n, m^{\prime} n^{\prime}}} \gamma_{m^{\prime} n^{\prime}, m n}
$$

donde $\beta=\frac{1}{k_{B} T}$ es el inverso de la temperatura.

Sustituyendo los operadores en la Ec. (10.13) encontramos que las poblaciones evolucionan de acuerdo con,

$$
\frac{d}{d t} \rho_{m n, m n}(t)=\sum_{m^{\prime} n^{\prime}} \gamma_{m n, m^{\prime} n^{\prime}} \rho_{m^{\prime} n^{\prime}, m^{\prime} n^{\prime}}(t)-\rho_{m n, m n}(t) \sum_{m^{\prime} n^{\prime}} \gamma_{m^{\prime} n^{\prime}, m n}
$$

Considerando los dos primeros niveles y las condiciones iniciales señaladas, obtenemos las poblaciones resolviendo las ecuaciones diferenciales acopladas,

$$
\begin{aligned}
& \frac{d}{d t} \rho_{00,00}(t)=\gamma_{00,10} \rho_{10,10}(t)-\gamma_{10,00} \rho_{00,00}(t) \\
& \frac{d}{d t} \rho_{10,10}(t)=\gamma_{10,00} \rho_{00,00}(t)-\gamma_{00,10} \rho_{10,10}(t) .
\end{aligned}
$$

Las coherencias evolucionan de acuerdo con,

$$
\rho_{m n, m^{\prime} n^{\prime}}(t)=\rho_{m n, m^{\prime} n^{\prime}}(0) e^{-i\left(E_{m n}-E_{m^{\prime} n^{\prime}}\right) t} e^{-\frac{1}{2}\left(\sum_{k l} \gamma_{k l m n}+\sum_{k l} \gamma_{k l m^{\prime} n^{\prime}}\right) t}
$$

de donde obtenemos,

$$
\begin{gathered}
\rho_{00,10}(t)=\frac{1}{2} e^{i \omega t} e^{-\frac{1}{2}\left(\gamma_{10,00}+\gamma_{02,00}+\gamma_{00,10}+\gamma_{02,10}\right) t} \\
\rho_{10,00}(t)=\frac{1}{2} e^{-i \omega t} e^{-\frac{1}{2}\left(\gamma_{10,00}+\gamma_{02,00}+\gamma_{00,10}+\gamma_{02,10}\right) t}
\end{gathered}
$$


podemos aproximar todas las $\gamma$ 's en términos de $\gamma_{00,10}$, la cual gobierna la velocidad de relajación desde el estado $|10\rangle$ al estado $|00\rangle$ (que es la mayor velocidad de relajación) por medio de las relaciones,

$$
\begin{aligned}
& \gamma_{10,00}=\quad \gamma_{00,10} e^{-\left(E_{10}-E_{00}\right)}=\gamma_{00,10} e^{-\omega}=\frac{\gamma_{00,10}}{e} \\
& \gamma_{02,00}=\gamma_{00,02} e^{-\left(E_{02}-E_{00}\right)}=\gamma_{00,02} e^{-2 \sqrt{\omega^{2}-2 \lambda^{2}}} \approx \frac{\gamma_{00,10}}{e^{2 \sqrt{\omega^{2}-2 \lambda^{2}}}} \\
& \gamma_{02,10}=\gamma_{10,02} e^{-\left(E_{02}-E_{10}\right)}=\gamma_{10,02} e^{\omega-2 \sqrt{\omega^{2}-2 \lambda^{2}}} \approx \frac{\gamma_{00,10}}{e^{-\omega+2 \sqrt{\omega^{2}-2 \lambda^{2}}}}
\end{aligned}
$$

\subsubsection{Localización en la densidad reducida}

En la Fig. (10.1) se muestra la evolución en el tiempo de la entropía de Shannon de la densidad reducida de una variable en el espacio de posición, $s_{\rho}$.

Las oscilaciones demuestran la localización/deslocalización que sufre el sistema en cada ciclo temporal $(2 \pi)$ mientras se aproxima al equilibrio. Las curvas correspondientes a los dos regímenes en que hemos resuelto la ecuación maestra de Lindblad e muestran juntas en la Fig. (10.1) sólo para enfatizar que las tendencias son muy similares y que algunas diferencias pueden ser captadas por la entropía de Shannon.
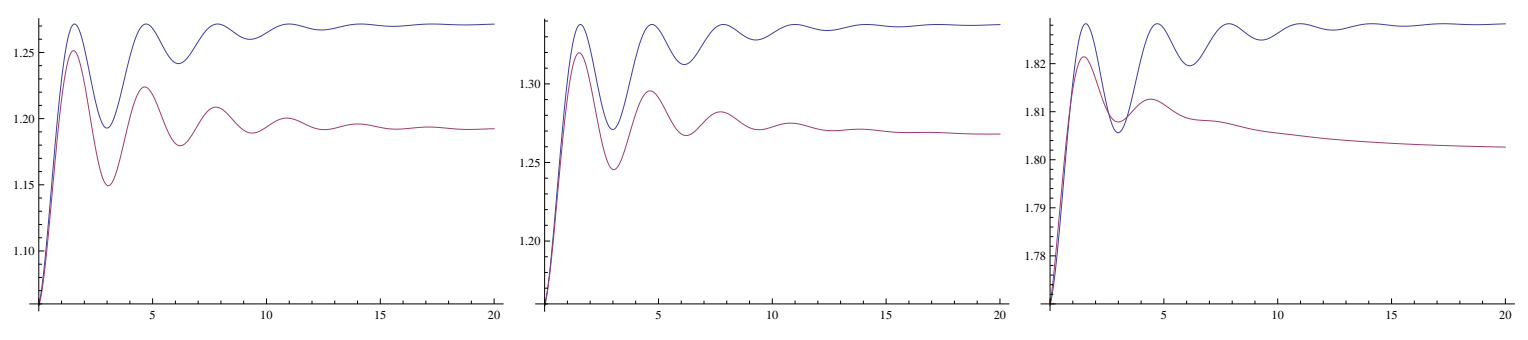

Figura 10.1: $s_{\rho}$ (ordenadas) como función del tiempo (abscisas) para el caso con desfasamiento puro sin relajación (azul) y para el caso con relajación sin desfasamiento puro (rojo). $\lambda=0.01$ (izquierda), $\lambda=0.5$ (centro), $\lambda=0.7$ (derecha).

En ambos límites de la ecuación de Lindblad las coherencias son cero para tiempos iguales a múltiplos semienteros de $\pi, t=n \pi / 2$, como se observa en la Fig. (10.1). En el caso de desfasamiento puro sin relajación no cambian las poblaciones y cada vez que las coherencias son cero tenemos una densidad que es igual en su forma a la 
mezcla estadística, donde las poblaciones son 1/2 y las coherencias han decaido, por lo que cada $t=n \pi / 2$ tenemos el valor de la entropía que caracterizaría a la mezcla estadística. En el caso de relajación sin desfasamiento puro el comportamiento de la entropía se debe al compromiso entre la decoherencia (cada $t=n \pi / 2$ ) y el cambio de las poblaciones que localiza el sistema.

El estado más localizado (con menor incertidumbre y por lo tanto con mayor información contenida en la densidad) en ambos casos es el estado inicial, que es un estado cuántico puro. En el caso de desfasamiento puro se observa que el estado correspondiente al equilibrio es el más deslocalizado, aunque como hemos señalado en el párrafo anterior ese valor de la deslocalización se obtiene periódicamente cada $t=n \pi / 2$. Por su parte el estado más deslocalizado del caso con relajación sin desfasamiento puro es con $t=\pi / 2$, y el estado en el equilibrio es más deslocalizado respecto al estado inicial, pero más localizado respecto del estado en $t=\pi / 2$.

La influencia del potencial entre partículas, $\lambda$, es delocalizar la densidad, como se aprecia al comparar las curvas en las tres gráficas de la Fig. (10.1).

\subsubsection{Localización en la densidad de pares}

En la Fig. (10.2) se muestra la evolución temporal de $s_{\Gamma}$, las entropías de pares en el espacio de posición. Las tendencias son muy similares a las entropías reducidas. La interpretación de las gráficas es el mismo.
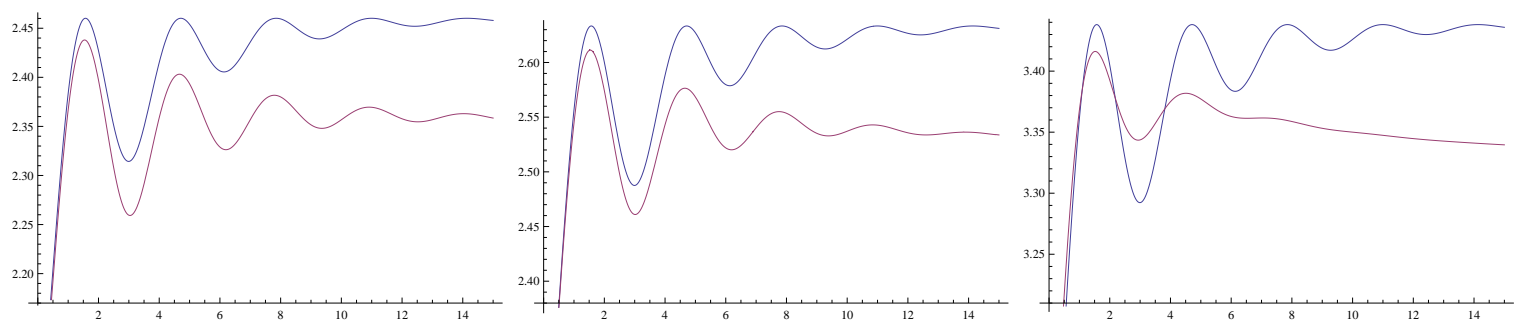

Figura 10.2: $s_{\Gamma}$ (ordenadas) como función del tiempo (abscisas) para el caso con desfasamiento puro sin relajación (azul) y para el caso con relajación sin desfasamiento puro (rojo). $\lambda=0.01$ (izquierda), $\lambda=0.5$ (centro), $\lambda=0.7$ (derecha). 


\subsubsection{Correlación estadística}

En la Fig. (10.3) se muestran las gráficas de la evolución temporal de la información mutua en el espacio de posición. En ambos casos de la ecuación de Lindblad la correlación oscila pero disminuye para valores mayores de $t$; en el caso con relajación sin desfasamiento puro parece disminuir más rápido que en el otro caso, para valores pequeños del potencial.

Las curvas en cada gráfica de la Fig. (10.3) no son comparables más que tendencialmente porque son dos regímenes distintos de interacción con el baño (los dos regímenes son independientes), sin embargo, es interesante que utilizando determinados valores fijos de $\gamma$, por separado en cada caso, el potencial entre partículas provoca un cambio en el orden de la magnitud de la correlación. Así, para los casos seleccionados, la correlación entre partículas es mayor en caso de desfasamiento puro sin relajación para valores pequeños del potencial entre partículas, $\lambda$, en tanto que para valores grandes de $\lambda$ la correlación es mayor en el caso de relajación sin desfasamiento puro.
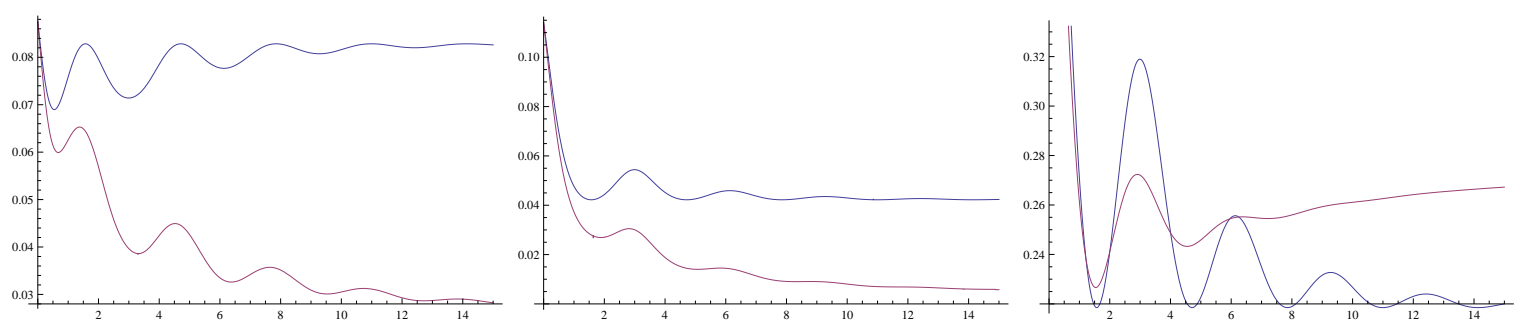

Figura 10.3: $I_{x}$ (ordenadas) como función del tiempo (abscisas) para el caso con desfasamiento puro sin relajación (azul) y para el caso con relajación sin desfasamiento puro (rojo). $\lambda=0.01$ (arriba), $\lambda=0.5$ (centro), $\lambda=0.7$ (abajo).

Por otro lado, el efecto del potencial en la correlación entre las posiciones de las partículas en cada uno de los regímenes se muestra en la Fig. (10.4). La influencia de una interacción entre partículas mayor es aumentar la correlación, como es de esperarse, pero también introduce un comportamiento oscilatorio más pronunciado en $I_{x}$. 

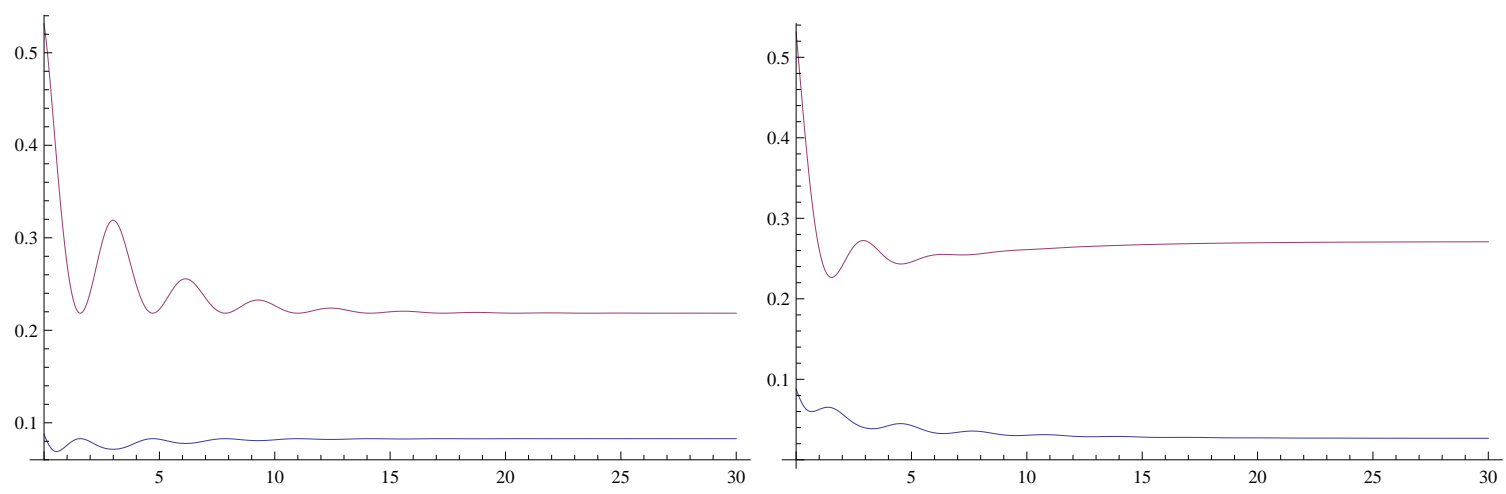

Figura 10.4: $I_{x}$, efecto del potencial, $\lambda=0.01$ (azul), $\lambda=0.7$ (rojo). Para el caso con desfasamiento puro sin relajación (izquierda), para el caso con relajación sin desfasamiento puro (derecha).

\subsubsection{Perspectivas sobre este modelo}

- Estudiar la localización en el espacio de momento a través de $s_{\rho}$ y de $s_{\Gamma}$.

- Estudiar las relaciones de incertidumbre como funciones del potencial entre partículas y como funciones del acoplamiento con el baño.

- Estudiar la correlación entre los momentos de las partículas.

- Estudiar la influencia del baño utilizando entropías relativas.

- Estudiar la evolución temporal del sistema utilizando entropías relativas.

- Estudiar la localización de las funciones de Wigner.

- Estudiar los efectos del baño térmico en la correlación posición-momento.

- También hemos comenzado a trabajar en la perspectiva de la Teoría de Funcionales de la Densidad Dependiente del Tiempo, extendiendo el trabajo para un oscilador armónico desarrollado en [156, 157] al átomo de Moshinsky, donde pueden estudiarse los efectos del acoplamiento con el baño sobre la interacción entre partículas. 


\section{Bibliografía}

[1] P. Matía-Hernando and A. Luis. Contradictions between different measures of quantum uncertainty. Physical Review A, 86:052106, 2012.

[2] H. Laguna. Correlación en los modelos de una y dos partículas en una caja. M.Sci. Thesis, Universidad Autónoma Metropolitana - Iztalapa, México, 2009.

[3] H.G. Laguna and R.P. Sagar. Indistinguishability and correlation in model systems. Journal of Physics A: Mathematical and Theoretical, 44:185302, 2011.

[4] Busch T., Englert B.-G., and Wilkens M. Rzazewski K. Two cold atoms in a harmonic trap. Foundations of Physics, 28:1998, 1998.

[5] G. Zürn, F. Serwane, T. Lompe, A. N. Wenz, M. G. Ries, J. E. Bohn, and S. Jochim. Fermionization of two distinguishable fermions. Physical Review Letters, 108:075303, 2012.

[6] Kiesel H., Renz A., and Hasselbach F. Observation of Hanbury Brown-Twiss anticorrelations for free electrons. Nature, 418:392-394, 2002.

[7] Jeltes T., McNamara J. M., Hogervorst W., Vassen W., Krachmalnicoff V., Schellekens M., Perrin A., Chang H., Boiron D., Aspect A., and Westbrook C. I. Comparison of the Hanbury Brown-Twiss effect for bosons and fermions. Nature, 445:402-405, 2007. 
[8] Neder I., Ofek N., Chung Y., Heiblum M., Mahalu D., and Umansky V. Interference between two indistinguishable electrons from independent sources. Nature, 448:333-337, 2007.

[9] Denschlag J. H. Quantum computing: Powered by symmetry. Nature, 448:422423, 2007.

[10] E.P. Wigner. On the quantum correction for thermodynamic equilibrium. Physical Review, 40:749-759, 1932.

[11] M. Hillery, R.F. O'Connell, M.O. Scully, and Wigner E.P. Distribution-functions in physics-fundamentals. Physics Reports, 106:121-167, 1984.

[12] V.I. Tatarskii. The Wigner representation of quantum mechanics. Uspekhi Fizicheskikh Nauk, 139:587-619, 1983.

[13] F. Pennini and A. Plastino. Localization estimation and global vs. local information measures. Physics Letters A, 365:263-267, 2007.

[14] F. Pennini, A. Plastino, G.L. Ferri, and F. Olivares. Semiclassical localization and uncertainty principle. Physics Letters A, 372:4870-4873, 2008.

[15] F. Olivares, F. Pennini, G.L. Ferri, and A. Plastino. Note on semiclassical uncertainty relations. Brazilian Journal of Physics, 39:503-506, 2009.

[16] B. Mirbach and H.J. Korsch. A generalized entropy measuring quantum localization. Annals of Physics, 265:80-97, 1998.

[17] S. Gnutzmann and K. Życzkowski. Renyi-Wehrl entropies as measures of localization in phase space. Journal of Physics A: Mathematical and Theoretical, 34:10123-10139, 2001.

[18] A.C. de la Torre and J.L. Iguain. Manifest and concealed correlations in quantum mechanics. European Journal of Physics, 19:563-568, 1998. 
[19] L. Henderson and V. Vedral. Classical, quantum and total correlations. Journal of Physics A: Mathematical and Theoretical, 34:6899, 2001.

[20] R. Shankar. Principles of Quantum Mechanics. Plenum Press, New York, 1994.

[21] J. J. Sakurai. Modern Quantum Mechanics. Addison-Wesley, USA, 1994.

[22] W. Heisenberg. "über den anschaulichen inhalt der quantentheoretischen kinematik und mechanik" ("the actual content of quantum theoretical kinematics and mechanics"). Zeitschrift für Physik, 43:172, 1927.

[23] H.P. Robertson. The uncertainty principle. Physical Review, 34:163-164, 1929.

[24] E. Schrödinger. HeisenbergAbout Heisenberg uncertainty relation. Proceedings of the Prussian Academy of Sciences, Physics-Mathematical section. quantph/9903100v2, XIX:296, 1930.

[25] D. Bohm. Quantum Theory. Dover, New York, 1989.

[26] M. Moshinsky. How good is the Hartree-Fock approximation. American Journal of Physics, 36:52, 1968.

[27] M. Moshinsky. "How good is the Hartree-Fock approximation," [am. j. phys. 36, 52 (1968)]. American Journal of Physics, 36:763, 1968.

[28] J.P. Dahl. Moshinsky atom and density functional theory - a phase space view. Canadian Journal of Chemistry, 87:784-789, 2009.

[29] R.J. Ya nez, A.R. Plastino, and J.S. Dehesa. Quantum entanglement in a soluble two-electron model atom. The European Physical Journal D, 56:141-150, 2010.

[30] C. Amovilli and N.H. March. Quantum information: Jaynes and Shannon entropies in a two-electron entangled artificial atom. Physical Review A, 69:054302, 2004. 
[31] Á. Nagy. Fisher information in a two-electron entangled artificial atom. Chemical Physics Letters, 425:154-156, 2006.

[32] A. Nagy and E. Romera. Relation between Fisher measures of information coming from pair distribution functions. Chemical Physics Letters, 490:242-244, 2010.

[33] H. G. Laguna and R. P. Sagar. Statistical correlations in the Moshinsky atom. Physical Review A, 84:012502, 2011.

[34] J. Pipek and I. Nagy. Measures of spatial entanglement in a two-electron model atom. Physical Review A, 79:052501, 2009.

[35] N.L. Harshman and W.F. Flynn. Entanglement in massive coupled oscillators. Quantum Information and Computation, 11:278-299, 2011.

[36] M.L. Glasser and I. Nagy. Exact evaluation of entropic quantities in a solvable two-particle model. Physics Letters A, 377:2317-2319, 2013.

[37] D. Manzano, A.R. Plastino, J.S. Dehesa, and T. Koga. Quantum entanglement in two-electron atomic models. Journal of Physics A: Mathematical and Theoretical, 43:275301, 2010.

[38] C.L. Benavides-Riveros, J.M. Gracia-Bondía, and J. C. Várilly. Lowest excited configuration of harmonium. Physical Review A, 86:022525, 2012.

[39] H.G. Laguna and R.P. Sagar. Position-momentum correlations in the Moshinsky atom. Journal of Physics A: Mathematical and Theoretical, 45:025307, 2012.

[40] K. Ebrahimi-Fard and J.M. Gracia-Bondía. Harmonium as a laboratory for mathematical chemistry. Journal of Mathematical Chemistry, 50:440-454, 2012.

[41] P. Blanchard, J.M. Gracia-Bondía, and J.C. Várilly. Density functional theory on phase space. International Journal of Quantum Chemistry, 112:1134-1164, 2012. 
[42] C. Amovilli and N.H. March. Exact density matrix for a two-electron model atom and approximate proposals for realistic two-electron systems. Physical Review A, 67:022509, 2003.

[43] A. Holas, I.A. Howard, and N.H. March. Wave functions and low-order density matrices for a class of two-electron 'artificial atoms' embracing hookean and Moshinsky models. Physics Letters A, 310:451-456, 2003.

[44] I. Nagy and J. Pipek. Hartree-Fock-like partitioning of the two-matrix for an exactly solvable two-electron model atom. Physical Review A, 83:034502, 2011.

[45] S. Ragot. Exact kohn-sham versus Hartree-Fock in momentum space: Examples of two-fermion systems. Journal of Chemical Physics, 125:014106, 2006.

[46] A. Akbari, N.H. March, and A. Rubio. Momentum density and spatial form of correlated density matrix in model two-electron atoms with harmonic confinement. Physical Review A, 76:032510, 2007.

[47] S. Ragot and M.B. Ruiz. Fourierlegendre expansion of the one-electron density matrix of ground-state two-electron atoms. Journal of Chemical Physics, 129:124117, 2008.

[48] H. Mera and K. Stokbro. Using kohn-sham density functional theory to describe charged excitations in finite systems. Physical Review B, 79:125109, 2009.

[49] T.A. Niehaus, N.H. March, and S. Suhai. Correlated and idempotent Dirac firstorder density matrices with identical diagonal fermion density: a route to extract a one-body potential energy in TDDFT. Journal of Mathematical Chemistry, 47:505-519, 2010.

[50] C.L. Benavides-Riveros and J.C. Várilly. Testing one-body density functionals on a solvable model. The European Physical Journal D, 66:274, 2012. 
[51] N.H. March, I.A. Howard, Á. Nagy, and A. Rubio. Pair function at coincidence and ground-state energy for interacting systems of two fermions with isotropic harmonic confinement and antiparallel spins. Physics Letters A, 288:101-104, 2001.

[52] C. Amovilli and N.H. March. Pair density related to one-electron information for the ground state of spin-compensated two-electron systems. International Journal of Quantum Chemistry, 102:132-138, 2005.

[53] I. Nagy, I. Aldazabal, and A. Rubio. Exact time evolution of the pair distribution function for an entangled two-electron initial state. Physical Review A, 86:022512, 2012.

[54] N.H. March, A. Cabo, F. Claro, and G.G.N. Angilella. Proposed definitions of the correlation energy density from a Hartree-Fock starting point: The twoelectron Moshinsky model atom as an exactly solvable model. Physical Review A, 77:042504, 2008.

[55] I. Nagy and J. Pipek. Approximations for the interparticle interaction energy in an exactly solvable two-electron model atom. Physical Review A, 81:014501, 2010.

[56] P.-F. Loos. Hooke's law correlation in two-electron systems. Physical Review A, 81:032510, 2010.

[57] M. Srednicki. Entropy and area. Physical Review Letters, 71:666, 1993.

[58] D. Han, Y.S. Kim, and M.E. Noz. Illustrative example of feynmans rest of the universe. American Journal of Physics, 67:61, 1999.

[59] S.K. Haldar and B. Chakrabarti. Dynamical features of Shannon information entropy of bosonic cloud in a tight trap. International Journal of Modern Physics $B, 27: 1350048,2013$. 
[60] J. P. Dahl. The Wigner function. Physica, 114A:439-444, 1982.

[61] Manfredi G. and Feix M. R. Entropy and Wigner functions. Physical Review E, 62:46654674, 2000 .

[62] Wlodarz J. J. Entropy and Wigner distribution functions revisited. International Journal of Theoretical Physics, 42:1075-1084, 2003.

[63] S. Aviyente and W. J. Williams. Entropy based detection on the time-frequency plane. Proceedings of ICASSP 2003, 6:441-444, 2003.

[64] K. Piatek and W. Leoński. Wehrl's entropy and a measure of intermode correlations in phase space. Journal of Physics A: Mathematical and Theoretical, $34: 4951,2001$.

[65] Ch. Kurtsiefer, T. Pfau, and J. Mlynek. Measurement of the Wigner function of an ensemble of helium atoms. Nature, 386:150-153, 1997.

[66] G. Nogues, A. Rauschenbeutel, S. Osnaghi, P. Bertet, M. Brune, J. M. Raimond, S. Haroche, L. G. Lutterbach, and L. Davidovich. Measurement of a negative value for the Wigner function of radiation. Physical Review A, 62:054101, 2000.

[67] J. P. Dahl and M. Springborg. Wigner's phase space function and atomic structure: I. the hydrogen atom ground state. Molecular Physics, 47:1001-1019, 1982.

[68] S. Nouri. Wigner phase-space distribution function for the hydrogen atom. Physical Review A, 57:1526-1528, 1998.

[69] J. P. Dahl and M. Springborg. Comment on "wigner phase-space distribution function for the hydrogen atom. Physical Review A, 59:4099-4100, 1999.

[70] L. Praxmeyer, J. Mostowski, and K. Wódkiewicz. Hydrogen atom in phase space: the Wigner representation. Journal of Physics A: Mathematical and General, 39:14143, 2006. 
[71] M. Springborg and J. P. Dahl. Wigners phase-space function and atomic structure: Ii. ground states for closed-shell atoms. Physical Review A, 36:1050-1062, 1987.

[72] M. Springborg. Wigner's phase space function and the bond in lih. Theoretica Chimica Acta, 63:349-356, 1983.

[73] H. Schmider and J. P. Dahl. Nodal structure of the electronic Wigner function of many-electron atoms and molecules. International Journal of Quantum Chemistry, 60:439-452, 1996.

[74] C.E. Shannon. A mathematical theory of communication. Bell System Technical Journal, 27:379-423, 1948.

[75] M. A. Nielsen and I. L. Chuang. Quantum Computation and Quantum Information. Cambridge University Press, Cambridge, UK, 2000.

[76] A. Khinchin. Mathematical foundations of information theory. Dover, New York, 1957.

[77] D. Applebaum. Probability and information : an integrated approach. Cambridge University Press, Cambridge UK, 2008.

[78] R. G. Parr and L. J. Bartolotti. Some remarks on the density functional theory of few-electron systems. The Journal of Physical Chemistry, 87:2810-2815, 1983.

[79] T.M. Cover and J.A. Thomas. Elements of Information Theory. John Wiley and Sons, New York, 1991.

[80] S. Kullback. Information Theory and Statistics. Dover, Gloucester, 1978.

[81] C. F. Matta, M. Sichinga, and P. W. Ayers. Information theoretic properties from the quantum theory of atoms in molecules. Chemical Physics Letters, 514:379$383,2011$. 
[82] H.H. Corzo, H.G. Laguna, and R.P. Sagar. Localization-delocalization phenomena in a cyclic box. Journal of Mathematical Chemistry, 50:233-248, 2012.

[83] V. Majerník, R. Charvot, and E. Majerníková. The momentum entropy of the infinite potential well. Journal of Physics A: Mathematical and Theoretical, 32:2207, 1999.

[84] M. Hô, D. F. Weaver, V. H. Smith Jr., R. P. Sagar, and R. O. Esquivel. Calculating the logarithmic mean excitation energy from the Shannon information entropy of the electronic charge density. Physical Review A, 57:45124517, 1998.

[85] M. Solimannejad and A. H. Pakiari. Calculating mean excitation energy from Shannon information entropy for kxe atoms. International Journal of Modern Physics C, 14:221, 2003.

[86] V. G. Tsirelson and Á Nagy. Binding entropy and its application to solids. The Journal of Physical Chemistry A, 113:9022-9029, 2009.

[87] R. O. Esquivel, A.L. Rodrguez, R. P. Sagar, M. Hô, and V. H. Smith Jr. Physical interpretation of information entropy: Numerical evidence of the collins conjecture. Physical Review A, 54:259-265, 1996.

[88] J. C. Ramírez, J. M. Hernández-Pérez, R. P. Sagar, R. O. Esquivel, M. Hô, and V. H. Smith Jr. Amount of information present in the one-particle density matrix and the charge density. Physical Review A, 58:35073515, 1998.

[89] P. Ziesche, V. H. Smith Jr., M. Hô, S. P. Rudin, P. Gersdorf, and M. Taut. The he isoelectronic series and the hookes law model: Correlation measures and modifications of collins' conjecture. The Journal of Chemical Physics, 110:6135, 1999.

[90] G. T. Smith, H. L. Schmider, and V. H. Smith Jr. Electron correlation and the eigenvalues of the one-matrix. Physical Review A, 65:032508, 2002. 
[91] L. M. Ghiringhelli, I. P. Hamilton, and L. Delle Site. Interacting electrons, spin statistics, and information theory. Journal of Chemical Physics, 132:014106, 2010.

[92] S. B. Trickey, V. V. Karasiev, and A. Vela. Positivity constraints and informationtheoretical kinetic energy functionals. Physical Review B, 84:075146, 2011.

[93] A. Grassi, G.M. Lombardo, N.H. March, and R. Pucci. 1/z expansion, correlation energy, and Shannon entropy of heavy atoms in nonrelativistic limit. International Journal of Quantum Chemistry, 69:721-726, 1998.

[94] S.R. Gadre, S.B. Sears, S.J. Chakravorty, and R.D. Bendale. Some novel characteristics of atomic information entropies. Physical Review A, 32:2602-2606, 1985.

[95] S.R. Gadre and R.D. Bendale. Information entropies in quantum chemistry. Current Science (India), 54:970-977, 1985.

[96] S.R. Gadre and R.D. Bendale. Rigorous relationships among quantummechanical kinetic energy and atomic information entropies: Upper and lower bounds. Physical Review A, 36:1932-1935, 1987.

[97] E. Romera and J.S. Dehesa. The FisherShannon information plane, an electron correlation tool. Journal of Chemical Physics, 120:8906, 2004.

[98] Q. Shi and S. Kais. Finite size scaling for the atomic Shannon-information entropy. Journal of Chemical Physics, 121:5611, 2004.

[99] K.D. Sen. Characteristic features of Shannon information entropy of confined atoms. Journal of Chemical Physics, 123:074110, 2005.

[100] K.D. Sen and J. Katriel. Information entropies for eigendensities of homogeneous potentials. Journal of Chemical Physics, 125:074117, 2006. 
[101] K.Ch. Chatzisavvas, Ch.C. Moustakidis, and C.P. Panos. Information entropy, information distances, and complexity in atoms. Journal of Chemical Physics, 123:174111, 2005.

[102] Q. Shi and S. Kais. Discontinuity of Shannon information entropy for two-electron atoms. Chemical Physics, 309:127-131, 2005.

[103] S.B. Liu. On the relationship between densities of Shannon entropy and Fisher information for atoms and molecules. Journal of Chemical Physics, 126:191107, 2007.

[104] Z. Huang and S. Kais. Entanglement as measure of electronelectron correlation in quantum chemistry calculation. Chemical Physics Letters, 413:1-5, 2005.

[105] K.Ch. Chatzisavvas, S.E. Massen, Ch.C. Moustakidis, and C.P. Panos. Applications of density matrices in a trapped bose gas. International Journal of Modern Physics B, 20:2189-2221, 2006.

[106] N.L. Guevara, R.P. Sagar, and R.O. Esquivel. Information uncertainty-type inequalities in atomic systems. Journal of Chemical Physics, 119:7030, 2003.

[107] Ch.C. Moustakidis, K.Ch. Chatzisavvas, and C.P. Panos. Theoretical quantuminformation properties of nuclei and trapped bose gases. International Journal of Modern Physics E, 14:1087-1104, 2005.

[108] N. L. Guevara. Estudio de entropías informacionales en sistemas atómicos. PhD Thesis., Universidad Autónoma Metropolitana - Iztapalapa, México, 2003.

[109] W. Kutzelnigg, G. Del Re, and G. Berthier. Correlation coefficients for electronic wave functions. Physical Review, 172:49-59, 1968.

[110] R.P. Sagar and N.L. Guevara. Mutual information and correlation measures in atomic systems. Journal of Chemical Physics, 123:044108, 2005. 
[111] R.P. Sagar and N.L. Guevara. Mutual information and electron correlation in momentum space. Journal of Chemical Physics, 124:134101, 2006.

[112] I. Bialynicki-Birula and J. Mycielski. Uncertainty relations for information entropy in wave mechanics. Communications in Mathematical Physics, 44:129-132, 1975.

[113] J.J. Halliwell. Correlations in the wave-function of the universe. Physical Review D, 36:3626-3640, 1987.

[114] A. Wehrl. General properties of entropy. Reviews of Modern Physics, 50:221-260, 1978.

[115] E. H. Allen. Negative probabilities and the uses of signed probability theory. Philosophy of Science, 43:53-70, 1976.

[116] J.P. Dahl, H. Mack, A. Wolf, and W.P. Schleich. Entanglement versus negative domains of Wigner functions. Physical Review A, 74:042323, 2006.

[117] J. Sperling and W. Vogel. Representation of entanglement by negative quasiprobabilities. Physical Review A, 79:042337, 2009.

[118] C. Usenko. Probabilistic aspects of the Wigner function. Optics and Spectroscopy, 99:404-410, 2005.

[119] K. Husimi. Some formal properties of the density matrix. Proceedings of the Physico-Mathematical Society of Japan, 22:264-314, 1940.

[120] A. N. Kolmogorov. Foundations of the Theory of Probability. Chelsea Pub. Co., New York, 1956.

[121] W. Mückenheim, G. Ludwig, C. Dewdney, P. R. Holland, A. Kyprianidis, J. P. Vigier, N. Cufaro Petroni, M. S. Bartlett, and E. T. Jaynes. A review of extended probabilities. Physics Reports, 133:337-401, 1986. 
[122] R. Feynman. Negative probability. B. Hiley, F. Peat, eds., Essays in Honor of David Bohm, Routledge and Kegan Paul, London, 0:235, 1987.

[123] J. B. Hartle. Quantum mechanics with extended probabilities. Physical Review A, 78:012108, 2008.

[124] S. N. Gupta. Quantum Electrodynamics. Gordon and Breach, London, 1977.

[125] N.L. Guevara, R.P. Sagar, and R.O. Esquivel. Shannon-information entropy sum as a correlation measure in atomic systems. Physical Review A, 67:012507, 2003.

[126] P.A Bouvrie, A.P. Majtey, A.R Plastino, P. Sánchez-Moreno, and J.S. De-hesa. Quantum entanglement in exactly soluble atomic models: the Moshinsky model with three electrons, and with two electrons in a uniform magnetic field. European Physical Journal D, 66:15, 2012.

[127] P. A. Bouvrie. Atomic information-theoretic measures and quantum entanglement of many-particle systems. PhD Thesis., Universidad de Granada, México, 2013.

[128] T. Koga and M. Sekiya. On the electron-electron counterbalance hole. Journal of Chemical Physics, 128:084105, 2008.

[129] T. Koga and H. Matsuyama. Note on the electron-electron counterbalance hole. Theoretical Chemistry Accounts, 126:383-385, 2010.

[130] W. Kohn. v-representability and density functional theory. Physical Review Letters, 51:1596-1598, 1983.

[131] R.P. Sagar, H.G. Laguna, and N.L. Guevara. Conditional entropies and positionmomentum correlations in atomic systems. Molecular Physics, 107:2071-2080, 2009.

[132] H.G. Laguna and Sagar R.P. Shannon entropy of the Wigner function and position-momentum correlation in model systems. International Journal of Quantum Information, 8:1089-1100, 2010. 
[133] M.F. Crommie, C.P. Lutz, and D.M. Eigler. Confinement of electrons to quantum corrals on a metal-surface. Science, 262:218-220, 1993.

[134] M.F. Crommie, C.P. Lutz, and D.M. Eigler. Imaging standing waves in a 2dimensional electron-gas. Nature, 363:524-527, 1993.

[135] M. Abramowitz and I. A. Stegun. Handbook of mathematical functions: with formulas, graphs, and mathematical tables. Dover, New York, 1970.

[136] J.S. Dehesa, A. Martínez-Finkelshtein, and V.N. Sorokin. Short-wave asymptotics of the information entropy of a circular membrane. International Journal of Bifurcation and Chaos, 12:2387-2392, 2002.

[137] H.H. Corzo, E. Castaño, H.G. Laguna, and R.P. Sagar. Measuring localizationdelocalization phenomena in a quantum corral. Journal of Mathematical Chemistry, 51:179-193, 2013.

[138] M. Belloni, M.A. Doncheski, and R.W. Robinett. Wigner quasi-probability distribution for the infinite square well: Energy eigenstates and time-dependent wave packets. American Journal of Physics, 72:1183-1192, 2004.

[139] V. G. Gueorguiev, A. R. P. Rau, and J. P. Draayer. Confined one-dimensional harmonic oscillator as a two-mode system. American Journal of Physics, 74:394, 2006.

[140] M. M. Almeida, M. N. Guimarães, and F. V. Prudente. Sobre o confinamento espacial de sistemas quânticos: O oscilador harmônico unidimensional e o átomo de hidrogênio. Revista Brasileira de Ensino de Física, 27:395-405, 2005.

[141] Ljiljana Stevanović. Fisher information and Shannon entropy in confined 1d harmonic oscillator. 7th International Conference of the Balkan Physical Union, pages 1188-1191, 2009. 
[142] J.L. Marin and S.A. Cruz. On the harmonic oscillator inside an infinite potential well. American Journal of Physics, 56:1134, 1988.

[143] J.L. Marin and S.A. Cruz. On the use of direct variational methods to study confined quantum systems. American Journal of Physics, 59:931, 1991.

[144] A.C. Tanner. The role of boundary conditions in separation of variables: Quantum oscillator in a box. American Journal of Physics, 59:333, 1991.

[145] G. Campoy, N. Aquino, and V. D. Granados. Energy eigenvalues and einstein coefficients for the one-dimensional confined harmonic oscillators. Journal of Physics A: Mathematical and General, 35:4903-4914, 2002.

[146] N. Aquino, E. Casta no, G. Campoy, and V. Granados. Einstein coefficients and dipole moments for the asymmetrically confined harmonic oscillator. European Journal of Physics, 22:645, 2001.

[147] K.D. Sen and A. K. Roy. Studies on the 3d confined potentials using generalized pseudospectral approach. Physics Letters A, 357:112-119, 2006.

[148] L. Stevanovic and K. D. Sen. Eigenspectrum properties of the confined 3d harmonic oscillator. Journal of Physics B: Atomic, Molecular and Optical Physics, 41:225002, 2008.

[149] H. E. Montgomery JR., N. A. Aquino, and K. D. Sen. Degeneracy of confined d-dimensional harmonic oscillator. International Journal of Quantum Chemistry, 107:798-806, 2007.

[150] S.M. Al-Jaber. A confined n-dimensional harmonic oscillator. International Journal of Theoretical Physics, 47:1853-1864, 2008.

[151] H. E. Montgomery Jr, G. Campoy, and N. Aquino. The confined n-dimensional harmonic oscillator revisited. Physica Scripta, 81:045010, 2010. 
[152] V. Majerník and L. Richterek. Entropic uncertainty relations for the infinite well. Journal of Physics A: Mathematical and Theoretical, 30:L49, 1997.

[153] A. Anderson. On predicting correlations from Wigner functions. Physical Review $D, 42: 585-589,1990$.

[154] R. Gebauer and R. Car. Current in open quantum systems. Physical Review Letters, 93:160404, 2004.

[155] A. Bodor and L. Diosi. Conserved current in markovian open-quantum systems. Physical Review A, 73:064101, 2009.

[156] D. G. Tempel. Time-Dependent Density Functional Theory for Open Quantum Systems and Quantum Computation. PhD. Thesis, Harvard University, USA, 2012.

[157] D. G. Tempel and A. Aspuru-Guzik. Relaxation and dephasing in open quantum systems time-dependent density functional theory: Properties of exact functionals from an exactly-solvable model system. Chemical Physics, 391:130142, 2011. 